University of Louisville

ThinkIR: The University of Louisville's Institutional Repository

$5-2012$

\title{
The role of acculturation and ethnic identity in understanding Asians' sport consumption behavior in the United States.
}

Jae-Pil Ha

University of Louisville

Follow this and additional works at: https://ir.library.louisville.edu/etd

\section{Recommended Citation}

$\mathrm{Ha}$, Jae-Pil, "The role of acculturation and ethnic identity in understanding Asians' sport consumption behavior in the United States." (2012). Electronic Theses and Dissertations. Paper 552.

https://doi.org/10.18297/etd/552

This Doctoral Dissertation is brought to you for free and open access by ThinkIR: The University of Louisville's Institutional Repository. It has been accepted for inclusion in Electronic Theses and Dissertations by an authorized administrator of ThinkIR: The University of Louisville's Institutional Repository. This title appears here courtesy of the author, who has retained all other copyrights. For more information, please contact thinkir@louisville.edu. 
THE ROLE OF ACCULTURATION AND ETHNIC IDENTITY IN

UNDERSTANDING ASIANS' SPORT CONSUMPTION BEHAVIOR IN THE UNITED STATES

\author{
By \\ Jae-Pil Ha \\ B.A., Gyeongsang National University, 2003 \\ M.S., University of Louisville, 2008 \\ A Dissertation \\ Submitted to the Faculty of the \\ College of Education and Human Development \\ in the University of Louisville \\ in Partial Fulfillment of the Requirements \\ for the Degree of
}

\begin{abstract}
Doctor of Philosophy
College of Education and Human Development

University of Louisville

Louisville, Kentucky
\end{abstract}

May 2012 
Copyright 2012 by Jae-Pil Ha

All rights reserved 
THE ROLE OF ACCULTURATION AND ETHNIC IDENTITY IN

UNDERSTANDING ASIANS' SPORT CONSUMPTION BEHAVIOR IN THE UNITED STATES

\author{
By \\ Jae-Pil Ha \\ B.A., Gyeongsang National University, 2003 \\ M.S., University of Louisville, 2008 \\ A Dissertation Approved on
}

February 24, 2012

by the following Dissertation Committee:

Mary A. Hams, Co-Chair

T. Christopher Greenwell, Co-Chair

Namok Choi

Anita M. Moorman

Krist King 


\section{DEDICATION}

This dissertation is dedicated to my loving wife, Hye-Young, who is the best painter I know. She has been with me every step throughout the process of this doctoral journey. Without her unwavering support and encouragement, I could not complete this journey. This is as much yours as it is mine.

I would also like to dedicate this work to my parents. Without their support and guidance, I would not be where I am today. I am forever indebted to my parents for all of their unconditional love. 


\section{ACKNOWLEDGEMENT}

It was a great honor for me to work on my dissertation with the best committee members. I must admit that this dissertation would not have been possible without sincere supports, advises, and encouragements of all my committee members. First of all, I would like to give many thanks my advisor, Dr. Mary A. Hums. She has been a true inspiration, fostering, and sharing my enthusiasm for this work. She has always taught and shown me how to become a real researcher as well as teacher. I cannot find any phrases to express my sincere appreciation for her. I'm truly and forever indebted for everything from her. Next, I would like to thank my co-chair, Dr. Christopher Greenwell, for patiently answering all of my questions, especially about survey designs and practical implications in my dissertation. Every single advice from him encouraged me to think critically and improved the quality of this work. Thanks Dr. Greenwell again for your kind and wholehearted support.

I would also like to express my deepest gratitude to other committee members. Thank you to Dr. Namok Choi for providing a statistical foundation at the University of Louisville and helping me navigate my way through Chapter III and IV. Thank you to Professor Anita Moorman for your warmest and heartfelt support and encouragement. I would also like to thank Dr. Kristi King for her endless support not just to me but also my wife, Hye-Young Go since day one in doctoral program. You have always been there for me and my wife, as a professor, as a mentor, and as a friend. 
I feel that I should acknowledge all of my colleagues, friends, and my students. Although I cannot specify their all names in this space, I would never forget what I had with them in my $\mathrm{PhD}$ journey. I would also acknowledge Dr. Nam-Gil Ha, who is my academic mentor since I have first met him 13 years ago. Without his sincere and constant encouragement, I cannot be here today.

My loving family, who have always sacrificed and prayed for me, would also deserve to receive acknowledgement. Particularly, words cannot express my gratitude to my parents, Chang Sik Ha and Dool Ryun Yoon, for all their endless and unwavering support.

Finally, none of this would have been possible without the support of the most important person in my life, Hye-Young Go. Hye-Young has certainly endured more than her share of quiet nights by herself as I sat in front of computer and attended class. Your sacrifice has allowed us to reach this goal together. I would never forget hand-made lunch box what she made every day for me. I thank her deeply. 


\title{
ABSTRACT \\ THE ROLE OF ACCULTURATION AND ETHNIC IDENTITY IN UNDERSTANDING ASIANS' SPORT CONSUMPTION BEHAVIOR IN THE UNITED STATES
}

\author{
Jae-Pil Ha
}

February 24, 2012

According to the U.S. Census Bureau (2011), ethnic minority populations in the U.S. constituted over one-third (approximately 126 million) of the total U.S. population in 2010 (approximately 300.8 million) (U.S. Census Bureau, 2011). With such rapidly increasing diversity of the U.S. population, sport marketers and researchers have become interested in targeting ethnic minority groups as niche markets to expand their fan bases. While the literature on sport consumption behaviors have solely focused on sociodemographic, individual, and psychographic factors, very limited attention was paid to cultural-related factors, such as acculturation and ethnic identity. Thus, the need to investigate the role of the two cultural factors in attracting various ethnic minorities to a sport consumption setting was identified.

The primary purpose of the current study was to develop sport consumption models for the Asian population living in the U.S., based on (a) ethnic identity, (b) acculturation, (c) identifications (points of attachment), and (d) consumption for the respective identification. Specifically, three different models were developed and tested according to types of identification [identification with an ethnic player (IEP), identification with a popular sport in a native country (IPSNC), and identification with a popular sport in a 
host country (IPSHC)] and types of consumption [consumption for a team with ethnic player (CTEP), consumption for a popular sport in a native country (CPSNC), and consumption for a popular sport in a host country (CPSHC). The current study also examined the impact of four acculturation strategies (integration, assimilation, separation, and marginalization) on identifications and consumption behaviors, based on Berry's (1990) bi-dimensional model of acculturation.

Survey data $(N=291)$ were collected from four Asian subgroups: (a) Chinese, (b) Japanese, (c) Korean, and (d) Taiwanese. The sport consumption models were then tested by structural equation modeling (SEM). Within all the three sport consumption models, ethnic identity and acculturation were negatively correlated. Ethnic identity significantly influenced identification in the Ethnic Player (IEP) and Native Sport (IPSNC) Models. However, ethnic identity did not directly influence consumption in all the three structural models. Acculturation significantly influenced only identification in the Host Sport Model (IPSHC). Regarding the relationship with consumption, acculturation only significantly influenced consumption in the Ethnic Player Model (CTEP). Lastly, within all the three structural models, identification significantly influenced consumption.

With respect to the acculturation strategies, the results revealed significant differences in the three identification and consumption factors among the four acculturation strategies. More specifically, Asians using integration and separation strategies were more likely to identify with and consume sport products reflecting attributes of their native countries (ethnic player and popular sports in a native country) than those using assimilation and marginalization strategies. For sport products reflecting attributes of the U.S. (popular sports in a host country), Asians using integration and 
assimilation strategies were more inclined to identify and consume the host popular sports than those using separation and marginalization strategies.

The current study extends the literature on ethnic minorities' sport consumption behavior, by incorporating the two cultural factors, ethnic identity and acculturation. Sport researchers and practitioners should further examine the two cultural factors to better understand sport consumption behaviors of ethnic minority groups. 


\section{TABLE OF CONTENTS}

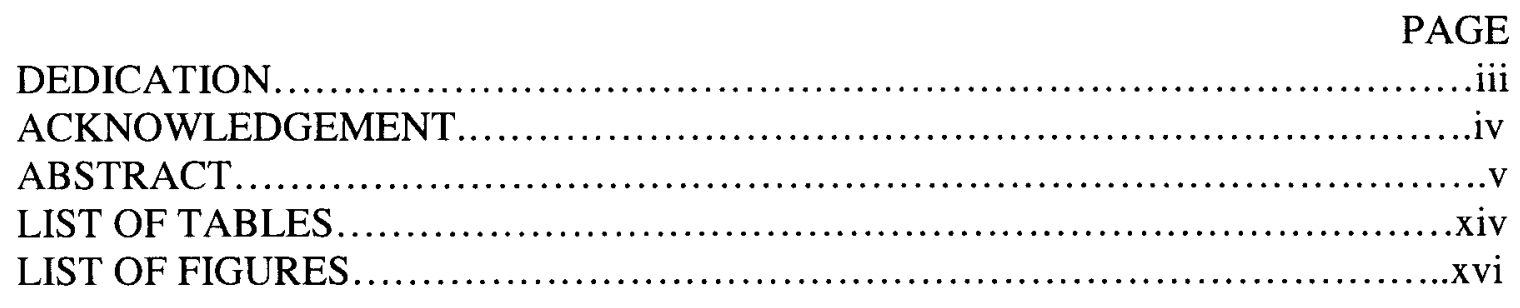

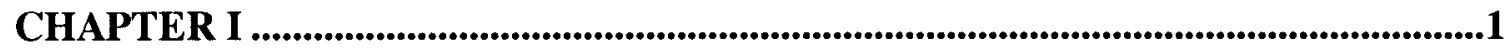

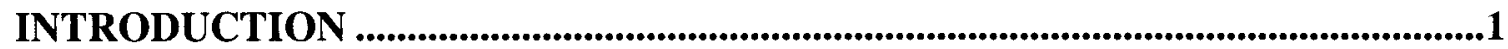

The Asian Market in the U.S. ............................................................................................4

Theoretical Grounding for the Current Study ..........................................................................7

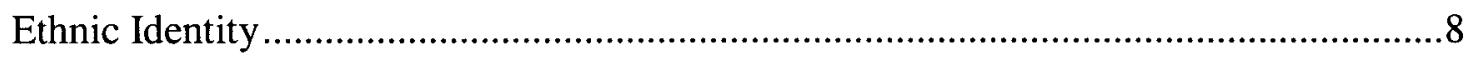

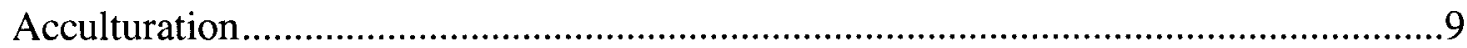

Fan Identification: Points of Attachment .................................................................

Statement of Problem .................................................................................................................13

Purpose of the Study .....................................................................................................................14

Significance of the Study ................................................................................................................16

Research Questions.........................................................................................................................................19

Delimitations ....................................................................................................................................................20

Limitations............................................................................................................................21

Definitions of Terms ....................................................................................................22

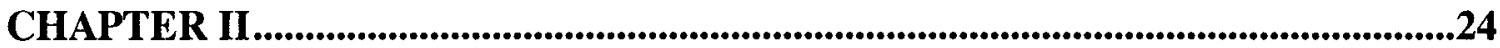




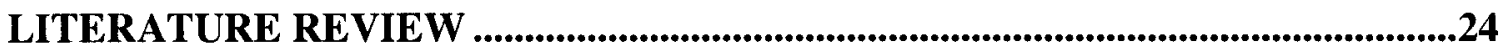

Attributes of the Asian Population in the U.S.....................................................................25

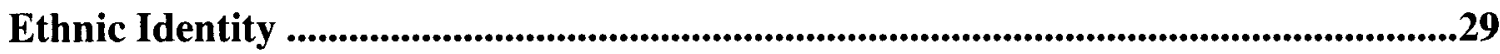

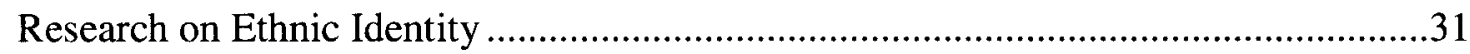

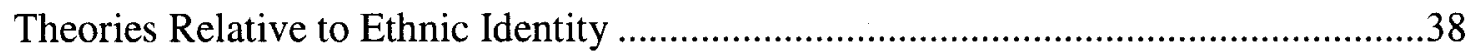

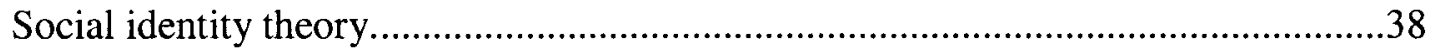

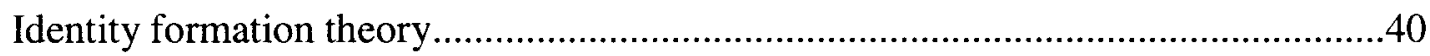

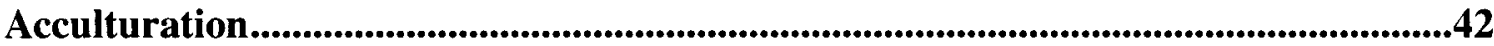

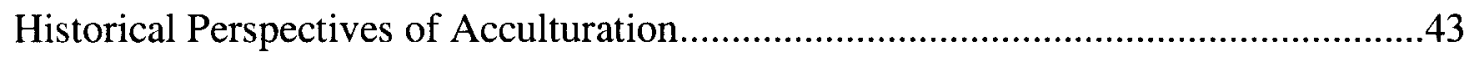

Two Levels of Acculturation (Group vs. Individual) ….................................................44

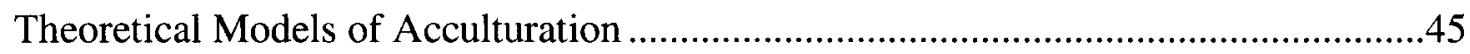

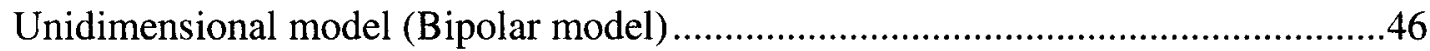

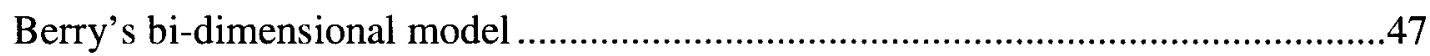

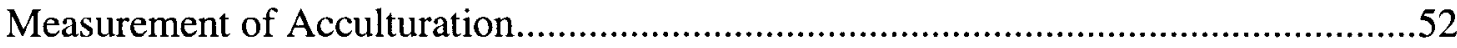

Personal and Environmental Factors Affecting Acculturation ....................................53

Consumption Behavior among Ethnic Minority Groups...............................................55

Summary

Sport Consumption Behavior among Ethnic Minority Groups....................................66

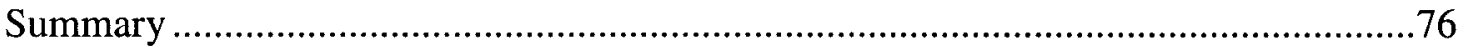

Ethnic Identity and Acculturation in a Sport Context...................................................77

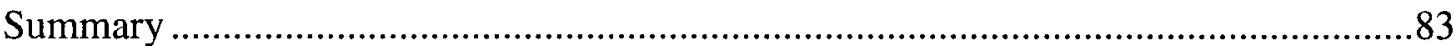

Identification in a Sport Setting .............................................................................84

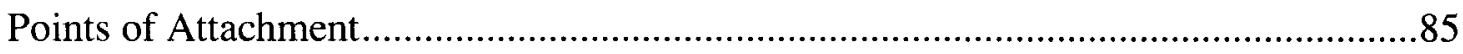

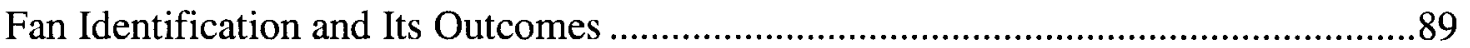

Athletic Achievement and Sport Consumption Behaviors ..........................................91 
Summary of Literature Review ........................................................................................................93

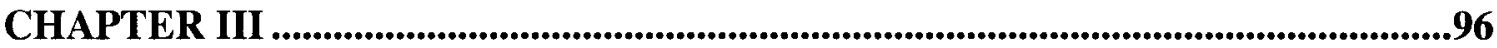

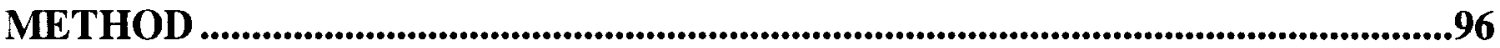

Purpose of the Study................................................................................................................96

Research Questions................................................................................................................99

Research Design ...............................................................................................................................................98

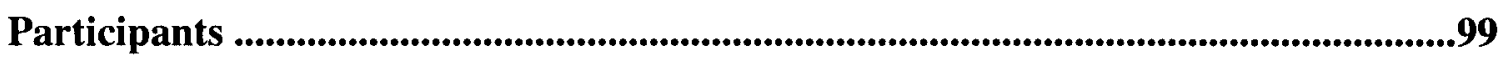

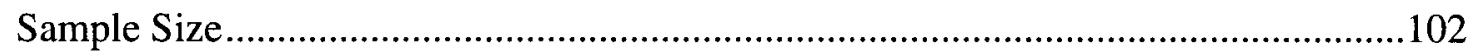

Data Collection Procedures .................................................................................................103

Instrumentation .......................................................................................................................105

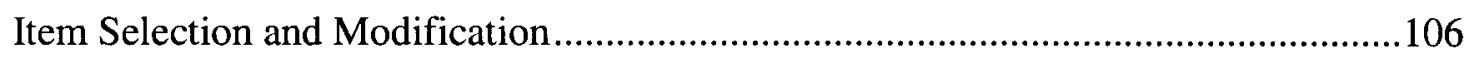

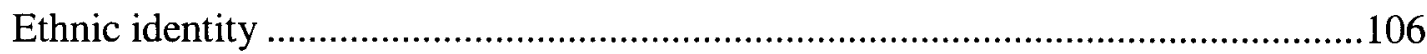

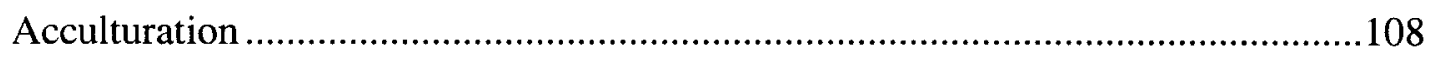

Sport fan identification ................................................................................111

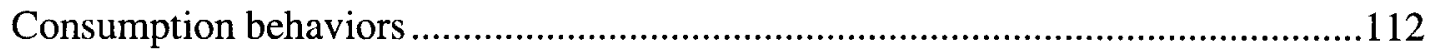

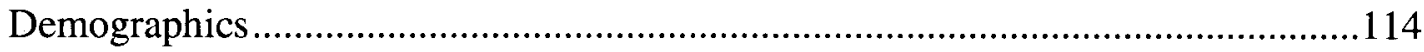

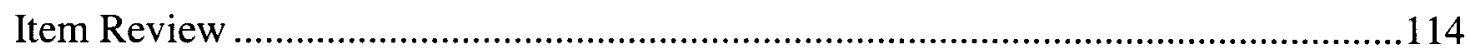

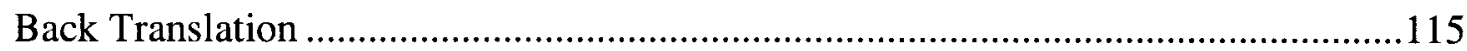

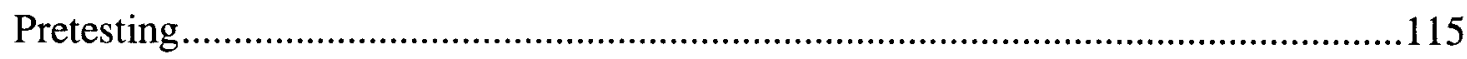

Data Analysis Procedures .....................................................................................116

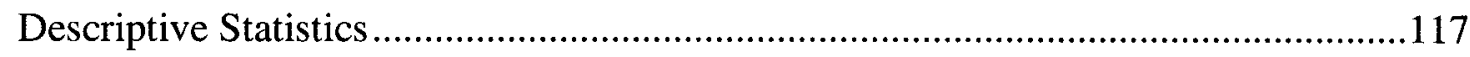

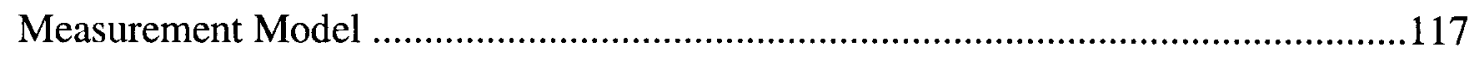

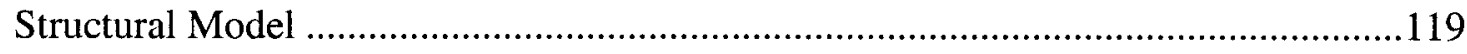

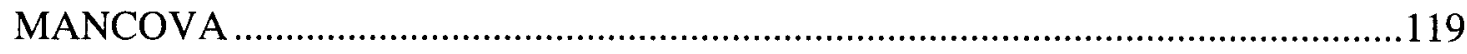

CHAPTER IV ..............................................................................................................................122 
RESULTS ...................................................................................................................................122

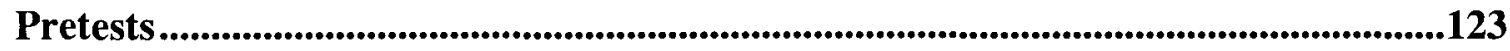

Panel of Expert

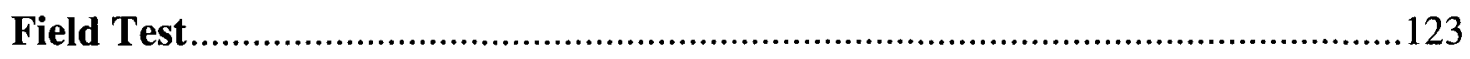

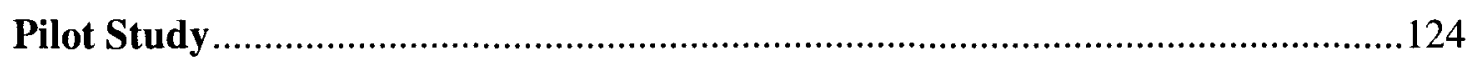

Sample Characteristics................................................................................................125

Preliminary Analysis ...............................................................................................129

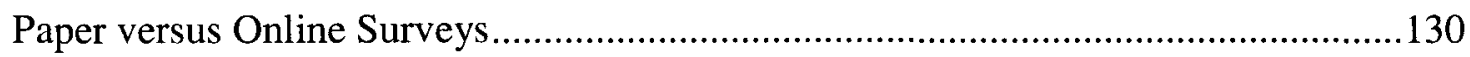

English versus Native Language Respondents .........................................................130

Skewness Check for Ethnic Identity and Acculturation .............................................131

Descriptive Statistics for Main Variables ........................................................................131

Measurement Models ...................................................................................................................133

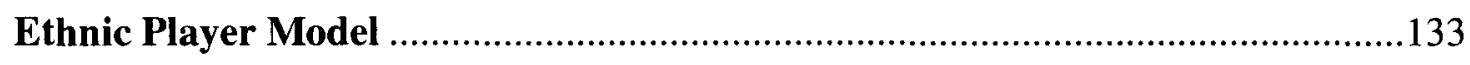

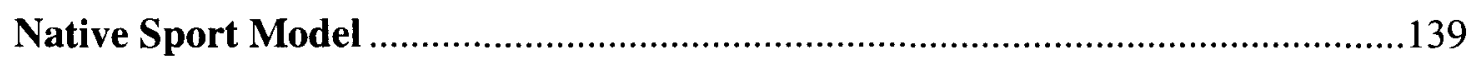

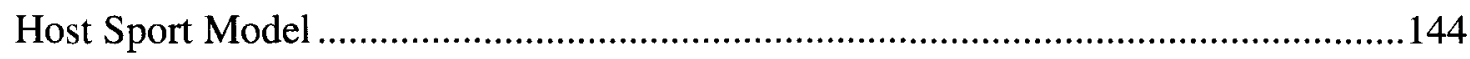

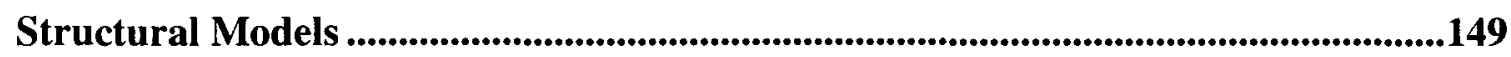

Ethnic Player Structural Model (Research Question 1).........................................149

Native Sport Structural Model (Research Question 2).........................................150

Host Sport Structural Model (Research Question 3) .........................................152

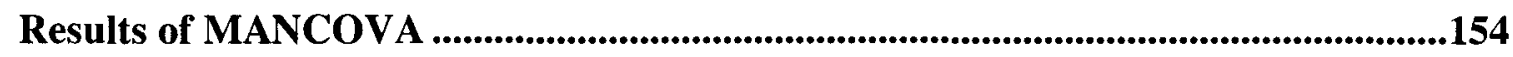

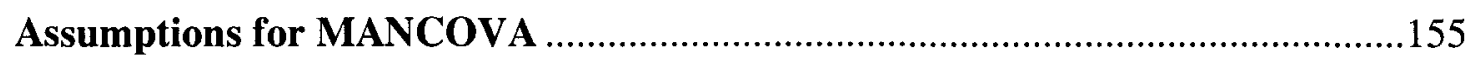

Research Question 4-1 (The Impact of Acculturation Strategies on IEP and CTEP) 
Research Question 4-2 (The Impact of Acculturation Strategies on IPSNC and CPSNC)

Research Question 4-3 (The Impact of Acculturation Strategies on IPSHC and CPSHC

Summary of Results.............................................................................................................................163

CHAPTER V ......................................................................................................................166

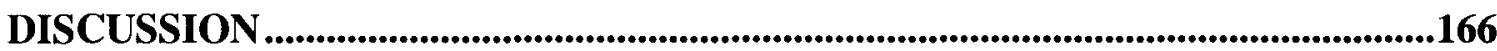

Major Implications of the Study .......................................................................................167

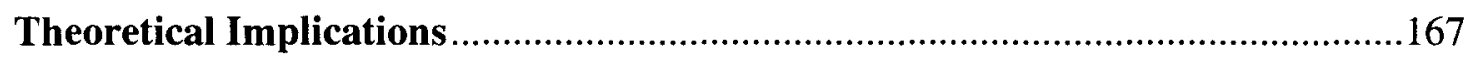

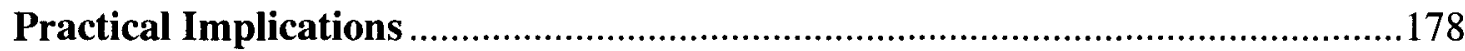

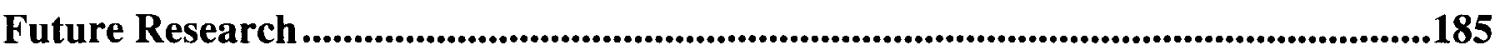

Summary of the Study

REFERENCES ...................................................................................................................................191

APPENDIX A.............................................................................................................................215

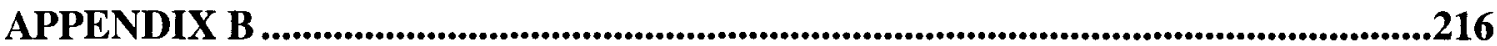

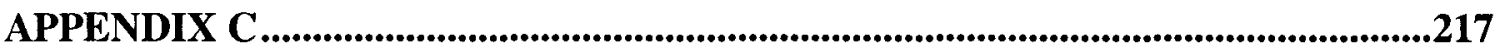

APPENDIX D ....................................................................................................................................229

CURRICULUM VITA .........................................................................................................231 


\section{LIST OF TABLES}

$\begin{array}{ll}\text { TABLE } & \text { PAGE }\end{array}$

1. Four acculturation strategies based on the AAMAS-CO and the AAMAS-

EA................................................................ 110

2. Cronbach's Alpha Coefficients $(\alpha)$ for All Subscales Included in the Survey

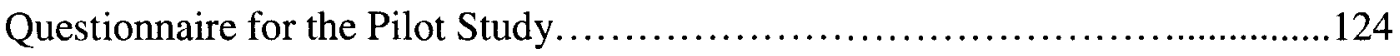

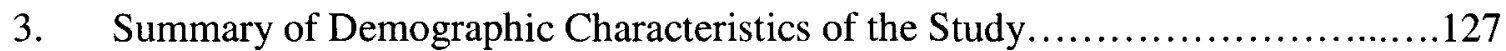

4. Means, Standard Deviations, Skewness, and Kurtosis for the Main Variables

5. Factor Loadings of the 17 items for Ethnic Player Measurement Mode................134

6. Factor Loadings $(\beta)$, Cronbach's Alpha Coefficients, and AVE for the Final Ethnic Player Measurement Model............................................... 138

7. Correlations among All the Four Factors for Ethnic Player Model................138

8. Factor Loadings $(\beta)$ of the 16 items for Native Sport Measurement Mode.........140

9. Factor Loadings $(\beta)$, Cronbach's Alpha Coefficients, and AVE for the Final Native Sport Measurement Model............................................... 143

10. Correlations among All the Four Factors for Native Sport Model................143

11. Factor Loadings $(\beta)$ of the 17 items for Host Sport Measurement Model..........144

12. Factor Loadings $(\beta)$, Cronbach's Alpha Coefficients, and AVE for the Final Host Sport Measurement Model 148 
13. Correlations among All the Four Factors for Host Sport Model................148

14. Summaries of Assumption Checks for MANCOVAs........................156

15. Pairwise Comparisons of Identification with Ethnic Player (IEP) by Acculturation Strategies........................................................ 158

16. Pairwise Comparisons of Consumption for a Team with Ethnic Player (CTEP) by Acculturation Strategies............................................ 158

17. Pairwise Comparisons of Identification with a Popular Sport in a Native Country (IPSNC) by Acculturation Strategies................................, 160

18. Pairwise Comparisons of Consumption for a Popular Sport in a Native Country (CPSNC) by Acculturation Strategies.................................... 160

19. Pairwise Comparisons of Identification with a Popular Sport in a Host Country (IPSHC) by Acculturation Strategies..................................... 162

20. Pairwise Comparisons of Consumption for a Popular Sport in a Host Country

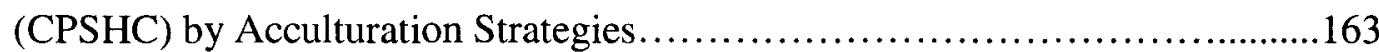

21. Summary of Results and Meanings for Research Questions

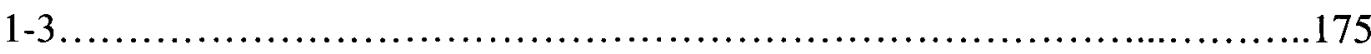

22. Summary of Results and Meanings for Research Question $4 \ldots \ldots \ldots \ldots \ldots \ldots \ldots 178$ 


\section{LIST OF FIGURES}

FIGURE

PAGE

1-1. A sport consumption model for Asian population based on identification with ethnic players............................................................

1-2. A sport consumption model for Asian population based on identification with a popular in a native country ...................................................

1-3. A sport consumption model for Asian population based on identification with a

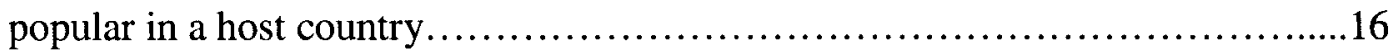

2. Bipolar model of acculturation or dual cultural unilinear model...................46

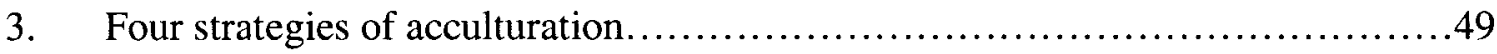

4. Acculturation strategies based on two dimensions-perspectives of nondominant

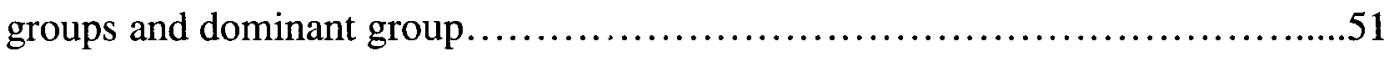

5. The Final Measurement Model for Ethnic Player..............................137

6. The Final Measurement Model for Native Sport.............................142

7. The Final Measurement Model for Host Sport.................................147

8. The Structural Model for Ethnic Player....................................150

9. The Structural Model for Native Sport Model......................................................152

10. The Structural Model for Host Sport Model.......................................................153 


\section{CHAPTER I}

\section{INTRODUCTION}

The sport industry has been considered one of the largest industries throughout the world, particularly in the United States (Plunkett, 2008). The estimated financial size of the U.S. sport industry is approximately $\$ 441.1$ billion per year. Although sporting goods (e.g., sporting equipment, apparel, athletic footwear) take a considerable amount of the current sport industry in the U.S., spectator sports are still a major part of the U.S. sport industry worth $\$ 28$ billion. More specifically, the "Big 4" leagues in North America (i.e., National Football League (NFL), Major League Baseball (MLB), National Basketball Association (NBA), and National Hockey League (NHL)) generated approximately $\$ 16.4$ billion annually (Plunkett, 2008).

The main element contributing to making the current sport industry valuable is sport consumers, including sport fans/spectators and sport participants. However, it is becoming more difficult for sport organizations to attract individuals into a sport, due to the fact that the current entertainment marketplace is relatively mature, highly competitive both within the sport industry and between the industry and other entertainment industries (e.g., movie, music, art) (Kim \& Trail, 2011; Park, Mahony, \& Greenwell, 2010). For example, over 1,000 collegiate athletic departments and 600 professional franchises in North America compete with each other for spectators' financial resources (Kim \& Trail, 2011). Further, the cost of operating a sport 
organization has increased far faster than its revenue (Kim \& Trail, 2011). Thus, it is critical for sport marketers and researchers to richen and more thoroughly understand various sport consumer behaviors, such as watching and attending sporting events, purchasing merchandise, and so forth. With the importance of sport consumers roles, numerous previous studies have examined a variety of socio-demographic, individual, cultural and psychographic factors affecting various sport consumption behaviors (e.g., Armstrong, 2002a, 2002b; Bilyeu \& Wann, 2002; Fink \& Parker, 2009; Fink, Trail, \& Anderson, 2002a, 2002b; Funk \& James, 2001, 2006; Funk, Mahony, \& Ridinger, 2002; Trail \& James, 2001; Trail, Robinson, Dick, \&Gillentine, 2003; Wann \& Branscombe, 1993). Despite the large amount of previous research on factors influencing sport consumer behaviors, few studies have examined cultural factors such as ethnic identity and acculturation. Understanding and examining the cultural factors that influence sport consumption behavior may offer both sport marketers and researchers meaningful information, considering that today's society is a multi-cultural and pluralistic society. According to Assael (1992), culture is the most powerful factor influencing consumption behaviors (e.g., food, clothing consumption), and people tend to purchase different products and services based upon their own cultural characteristics. Thus, various ethnic minorities' consumption behaviors can be naturally understood by examining cultural factors (e.g., acculturation, ethnic identity). In this regard, it is also critical for sport managers to effectively understand diverse sport consumption behaviors of ethnic minority individuals using the cultural factors. This knowledge could lead sport practitioners to expand their fan bases by targeting ethnic minority individuals as niche markets. 
With the rapidly increasing diversity of the U.S. population and marketers' increasing need to focus on minority groups as niches, numerous studies have been conducted on minorities' consumption behavior in the U.S. (e.g., Cleveland, Laroche, Pons, \& Kasto, 2009; Jun, Ball, \& Gentry, 1993; Penaloza, 1994). Taylor (2001, cited in Shafritz, Ott, \& Jang, 2010, p. 492) concluded that, "because research on consumer behavior has consistently shown, socio-cultural identities affect buying behavior, marketing success will depend... on the ability of companies to understand and respond effectively to the cultural nuances of the diverse marketplace."

Culture is recognized as the most important influence on consumption patterns/behaviors among ethnic minority groups (Cleveland \& Laroche, 2007). Due to the varying values, lifestyles, and cultural backgrounds of different ethnic groups, it is evident that there are differences in consumption behaviors/patterns across cultures (Cleveland et al., 2009). Assael (1992) found that culture is the most powerful factor affecting consumer behavior, and cultural differences are reflected in products and services purchased. In other words, similar consumption behaviors seem to emerge for consumers with a certain ethnic background, such as selecting similar products or services appropriate for their particular needs and desires. Consumers from different ethnic backgrounds, although sharing norms and values of the host culture, are likely to show certain different consumption behaviors from individuals of other ethnic groups (Kim, Laroche, \& Joy, 1990). Thus, differential marketing efforts are likely to be more effective in responding to their different needs and desires. From this point of view, we can infer that a certain ethnic group would prefer to watch, attend, or participate in a specific sport reflecting one's own cultural identity. 


\section{The Asian Market in the U.S.}

The total population of people in the U.S. who self-identified as being Asian or Asian-related races was17.3 million in 2010 (U.S. Census Bureau, 2011). The anticipated percentage increase in the number of Asians in the U.S. between 2000 and 2050 is a significantly large $213 \%$. This is more than five times the projected increase of the U.S. total population (49\%) over the same period of time (Le, 2010a). The U.S. Census Bureau (2011) indicated the total Asian population increased by $43 \%$ between 2000 and 2010, which was one of the fastest rates of growth among ethnic groups, while the White population had the slowest rate of the growth during the same period. Further, the Selig Center for Economic Growth reported that the nation's Asian purchasing power is anticipated to increase by over 600\% from $\$ 116$ billion in 1990 to $\$ 697$ billion in 2014 (Humphreys, 2009). Humphreys projected that the buying power of Asian Americans will grow at a more rapid rate than that of any other ethnic minority group except for Hispanics. Finally, the 2005 median household income for Asians in the U.S. was $\$ 64,238$, the highest among all ethnic minority groups (Le, 2010a).

In spite of the growth of the Asian market in the U.S., the sport consumption level of the Asian population in the U.S. is still relatively low. For instance, Asian Americans represented less than $2 \%$ of total fans in each of several professional sport leagues. In 2002, the percentage of Asian American fans for various professional sporting events was as follows: MLB, 0.9\%; NHL, 1.2\%; NBA, 1.3\%; NFL, 1.0\%; Major League Soccer (MLS), 1.6\%; and National Association for Stock Car Auto Racing (NASCAR), 0.7\%. However, in the same year, Hispanics and African Americans showed much greater levels of fan/spectator participation, at $11.8 \%$ and $10.8 \%$ for MLB, $10.2 \%$ and $8.4 \%$ for 
the NHL, $14.5 \%$ and $18.3 \%$ for the NBA, $11.2 \%$ and $13.0 \%$ for the NFL, $19.2 \%$ and $11.2 \%$ for MLS, and $10.6 \%$ and $9.6 \%$ for the NASCAR, respectively (Spanberg, 2003). With regard to Asian athletes, merely $2.3 \%$ of MLB athletes, $1.0 \%$ of NBA players, and $2.0 \%$ of NFL players were Asians, according to the 2010 Racial and Gender Report Card (Lapchick, Kaiser, Caudy, \& Wang, 2010; Lapchick, Kaiser, Russell, \& Welch, 2010; Lapchick, Kitnurse, \& Moss II, 2010). These figures revealed that the Asian sport market in the U.S. is relatively less developed compared to two other ethnic minority groups (i.e., African Americans and Hispanics). Hence, the Asian population may be regarded as an underdeveloped market segment possessing great growth potential (Ko, Claussen, Rinehart, \& Hur, 2008). Considering that one of the major objectives of sport marketers is to develop new sport fans (Park, Andrew, \& Mahony, 2008), the Asian population in the U.S. offers sport marketers great opportunities for expanding fan bases and increasing sales revenues. However, it is surprising that recent research on sport consumption behavior of ethnic groups in the U.S. has overlooked the Asian market while primarily focusing on African Americans and Hispanics (Ko et al., 2008). Given Asian athletes in the U.S. may draw more Asian fans in a stadium or arena (Wang \& Zhang, 2010), it is important for professional sport franchises to recruit high-profile Asian athletes.

Despite this potential, one primary stereotype that seems to hinder marketing to the Asian American population is that all Asian Americans are the same. In other words, many people are unable to distinguish different Asian ethnic groups (e.g., Korean Americans from Chinese Americans). Making generalizations of certain beliefs regarding one or a few Asian Americans to the whole Asian American population can become a marketing problem, considering that Asian Americans are composed of more than 30 
different ethnic subgroups, each of which has its own culture (Kaufman-Scarborough, 2000). Thus, it should come as no surprise that there are large cultural differences in the context of consumer behavior among various Asian subgroups in the U.S. However, according to Magazine Publishers of America (2004), marketing and advertising agencies have typically divided Asian populations into three segments to strengthen their efforts in reaching the diverse Asian subgroups based on their regional and cultural closeness: (a) Northeast Asians (e.g., Chinese, Korean, Japanese, Taiwanese, Vietnamese), (b) Southeast Asians (e.g., Filipino, Malaysian, Indonesian, Cambodian), and (c) South Asians (e.g., Indian, Pakistani, Bangladeshi). From a marketer's standpoint, this group market segmentation of Asian may be more proper than individually dividing a variety of Asian subgroups due to its cost-effectiveness in terms of management of various subgroups.

Of the more than 30 different ethnic subgroups comprising the Asian populations, three representative subgroups that have great economic power are the Chinese, Korean, and Japanese ("Asian-American Market in the U.S.," 2008). In addition, the sport industry in those three countries is relatively developed compared to other Asian countries. Another country in which the sport industry is relatively developed is Taiwan. Thus, people moving from those four countries (China, Japan, Korea, Taiwan) to the U.S. may be accustomed to watching or participating in sports as an entertainment option. It is also important to note that when marketing agencies and advertisers segment various subgroups of Asian population in the U.S., people from these four countries belong to Northeast Asian group due to their geographical or cultural proximity (Magazine Publishers of America, 2004). Thus, the current study focused on the Asian populations 
whose culture of origin is one of those four countries. However, for the purpose of this study, the four subgroups (China, Japan, Korea, Taiwan) were not directly compared to each other. Rather, data were analyzed as one whole group, since all four subgroups belong to the Northeast Asian region (see Chapter III).

As trade barriers between countries decrease significantly, numerous professional sport teams in the U.S. have begun to recruit international players and then utilize the players as a part of their marketing strategy to target a specific ethnic group. This phenomenon is related to the concept of "Glocalization". The term glocalization refers to "the tailoring and advertising of goods and services on a global or near-global basis to an increasingly differentiated local and particular market" (Robertson, 1995, p. 28). In recent years, North American professional sports leagues, particularly MLB and NBA, are attempting to engage in glocalization through marketing their products locally (e.g., marketing Korean player to Koreans in the U.S.) and globally (e.g., marketing Korean players to those in Korea) to target ethnic minority groups after recruiting high-profile athletes of various nationalities. In particular, considering the importance of the Asian population from the four countries listed above, many MLB and NBA teams have recruited high-profile athletes from the four countries. Ichiro Suzuki of Seattle Mariners and Yao Ming of the Huston Rockets are the best examples. This marketing strategy by North American professional sport teams may bring about increased fan-athlete identification and eventually extend fan bases and generate more ethnic-specific revenue.

\section{Theoretical Grounding for the Current Study}

Numerous studies have examined general consumer behaviors (e.g., food, clothing) with various ethnic minority groups (e.g., Hispanic, African American, and Asian 
American) in cross-cultural consumer and marketing-related journals. Two major constructs on which ethnic minorities' consumption behaviors are based are ethnic identity and acculturation. These two constructs can be useful tools in explaining sport consumption behavior among ethnic minority groups. In addition to these two constructs, one of the important determinants of sport consumption behavior is fan identification.

\section{Ethnic Identity}

The term ethnic identity incorporates several aspects of self-identification, such as a sense of belonging and commitment to a group, a sense of values and attitudes toward one's ethnic group, and participation in a range of cultural activities. These activities include participation in shared language, behaviors, friendship, religion, politics, and any combination of these (Persky \& Birman, 2005; Phinney, 1990, 1996; Phinney, Horenezyk, Libkind, \& Vedder, 2001). Although ethnic identity has been defined in numerous ways, one encompassing definition that appears to capture most is "an enduring, fundamental aspect of the self that includes a sense of membership in an ethnic group and the attitudes and feelings associated with that member [ship]" (Phinney, 1996, p. 922).

Within the framework of social identity theory, ethnic identity has been regarded as one portion of a person's general social identity that draws from membership in the individual's ethnic group, and brings the value and emotional worth attached to that membership (Tajfel, 1981). Phinney (1990) defined ethnic identity as an individual's retention and attainment of cultural uniqueness that is integrated into the self-concept, which develops within the individual as a member of a minority ethnic group within the dominant society. Social identity theory has been consistently employed by researchers to 
examine the complexities people experience from negotiating two cultures. These complexities often result in immigrants facing conflicts between behaviors, values, and attitudes of their culture of origin and those of the dominant culture.

\section{Acculturation}

Stephenson (2000) defined acculturation as "a complex, multidimensional process of learning that occurs when individuals and groups come into continuous contact with a different society" (p.77). Acculturation theory has been widely used to explain the adaptation patterns of newcomers (e.g., immigrants, sojourners, refugees). This theory explains how newcomers develop strategies in order to adapt to a new or different society (Berry, 1990, 1997). According to a thorough review of literature on acculturation, particularly in psychology and cross-cultural research, the acculturation process has evolved from a unidimensional process (also called unidirectional and unilinear or bipolar) to a bidimensional process (also called bidirectional and two-dimensional). From the unidimensional perspective, individuals' adaptations to the dominant culture are viewed as necessarily accompanied by a weakening of ties to one's culture of origin (Nguyen, Messe, \& Stollak, 1999). As such, the culture of origin and the dominant culture are negatively correlated with each other. The unidimensional acculturation process can be described as a single continuum. That is, individuals in one end of the continuum (unacculturated people) maintain their original cultural values, attitudes, beliefs, and behaviors, but reject those of the dominant culture, whereas individuals in the other end of the continuum (acculturated people) accept cultural values, attitudes, and behaviors of the dominant culture, but lose those of the original culture. Although acculturation model has evolved from the unidimensional to the bi-dimensional, some 
researchers still tend to conceptualize and measure individuals' acculturation process with the unidimensional model (e.g., Cuellar, Arnold, \& Maldonado, 1995; Landrine \& Klonoff, 1994).

In contrast, the bi-dimensional model assumes that newcomers are capable of accepting two different sets of cultural values. This model takes into account orientation to both original and dominant cultures simultaneously (Berry, 1990, 1997, 2003). The bidimensional model assumes that the strength of orientation toward one's own culture and toward the dominant culture of the nation in which one lives should be regarded as relatively independent and essentially orthogonal rather than interdependent.

Based upon this bi-dimensional model of acculturation, Berry $(1990,1997)$ suggested four different types of acculturation strategies: (a) integration, (b) assimilation, (c) separation, and (d) marginalization. The integration strategy/pattern occurs when individuals retain their own cultural values and identity while accepting those of the dominant culture. Thus the integration strategy represents biculturalism. The assimilation strategy is the process by which immigrants relinquish their culture and accept a new culture. The separation strategy occurs when individuals wish to accept their own cultural values and identity while avoiding the dominant cultural values. The marginalization strategy occurs when immigrants lose their relationship with both their original and new culture.

Even though the constructs of acculturation and ethnic identity have been widely used in general consumer behavior research, few researchers to date have examined the two constructs in sport management and marketing (Harrole \& Trail, 2007; Pons, Laorche, Nyeck, \& Perreault, 2001). Pons et al. (2001) first investigated how ethnic 
identity and acculturation impact sporting events consumption and orientation among Italian Canadians. The results of their study indicated that identification with and consumption orientation toward a certain sport was influenced by both ethnic identity and acculturation. On the other hand, Harrole and Trail (2007) found that acculturation and ethnic identity had little impact on identification with sports. Unlike the two studies, Shin (2010) applied the construct of acculturation into sporting goods (i.e., golf equipments) and found that the level of acculturation affected golf consumers' decision-making styles.

\section{Fan Identification: Points of Attachment}

The construct of sport fan identification is a unique attribute distinguishing sport from other sources of entertainment since the degree of involvement and affiliation with sport-related objects (e.g., teams, players, teams, sports itself) is much more variable than the degree of involvement with other forms of entertainment. Due to this unique attribute, sport fan identification to date is a primary theme in the study of sport consumption behavior. Trail, Anderson, and Fink (2000) defined "identification as an orientation of the self in regard to other objects including a person or group that results in feelings or sentiments of close attachment" (p.165-166). Early research on sport fan identification focused on the effects of a fan's psychological connection to a specific team, which is called team identification (e.g., Branscomb \& Wann, 1991; Sutton, McDonald, Milne, \& Cimperman, 1997; Wann \& Branscomb, 1990, 1993). However, some researchers have begun to consider additional identifications beyond team identification within sport, suggesting fans might also identify with other sport-related objects, including the player, the coach, the community, the university, and the sport itself (Robinson \& Trail, 2005; Trail, Robinson, Dick, \& Gillentine, 2003). From this perspective, a sport team is just one 
point of attachment for identification. In other words, sport fans not only identify with a team ,but also identify with other sport-related objects above.

It is important to note that since point of attachment and identification are frequently used interchangeably in sport consumption behavior literature; both terms are used in this study. For reference, points of attachment refer to one's psychological attachment to diverse aspects of sports (e.g., sport itself, player, coach).

Research on points of attachment, in addition to team identification, is now an emerging line of inquiry in sport fan consumption behavior. Little has been investigated, though, about how ethnic minority groups identify with other sport-related objects including players and sports. According to Min and Kim (2009), Asian immigrants often used sport as a tool to express the degree of their ethnic identity with native countries by supporting native players in the Olympic Games. Furthermore, Ko et al. (2008) suggested that sport organizations need to more actively recruit high-profile Asian players as a way of building fan-ethnic player identification, resulting in attracting more Asian American sport fans. Thus, it may be true that Asian immigrants are more likely to identify with native players and eventually attend and watch the team's game in which the athletes are playing.

In addition to identification with players, Pons et al. (2001) and Lee (2005) suggested that a specific ethnic group is likely to consume and associate with a certain specific type of sport. In other words, "a specific sport can be identified as being ethnically specific" (Pons et al., 2001, p. 235), such as ice hockey to Canadians, soccer to Brazilians, Taekwondo to Koreans, football to Americans, and so on. This demonstrates that attachment to a specific sport might vary depending on ethnic groups. Applying this 
concept to attachment to a popular sport in both native country and host country, it is possible that ethnic minority groups living in the U.S. may be more likely to identify with the popular sports in their native country than the host country and eventually read more articles about and watch the popular sports in the native country through the Internet and TV respectively. For example, an international college student (e.g., Korean) studying in the U.S. might identify more strongly with Korean professional baseball and follow news about that sport more than the popular sports in the host country (the U.S.), such as NBA, MLB, and NFL.

\section{Statement of Problem}

According to the U.S. Census Bureau (2011), ethnic minority populations in the U.S. constitute over one-third (approximately 126 million) of the total U.S. population in 2010 (approximately 300.8 million). This means ethnic minority groups can no longer be viewed and treated as marginal populations (Venkatesh, 1995). Of various ethnic groups, the Asian population in the U.S. should be regarded as an important consumer segment, given the rapid growth of this population and its economic potential (i.e., buying power, high mean household income). Despite the importance of the Asian population in the U.S., sport management practitioners and researchers have given relatively little attention to this ethnic group (Ko et al., 2008) while focusing on Hispanics and African Americans. In addition, little is understood about how cultural-related factors (i.e., ethnic identity and acculturation) affect their identification with sports or players, and their consumption behavior of Asian Americans. 
Therefore, to better understand sport consumption behaviors of Asian population living in the U.S., it is necessary to examine points of attachment and their sport consumption behaviors in relation to the constructs of ethnic identity and acculturation.

\section{Purpose of the Study}

There were two purposes for the current study. The first purpose was to develop and test Sport Consumption Models for the Asian population living in the U.S. based on ethnic identity and acculturation. For this purpose, this study explored the relationships among ethnic identity, acculturation, three points of attachment [identification with an ethnic player (IEP), identification with a popular sport in a native country (IPSNC), and identification with a popular sport in a host country (IPSHC)], and three sport consumption behaviors [consumption for a team with an ethnic player (CTEP), consumption for a popular sport in a native country (CPSNC), and consumption for a popular sport in a host country (CPSHC). The three proposed models for this purpose were presented in Figures 1-1 (Ethnic Player Model), 1-2 (Native Sport Model), and 1-3 (Host Sport Model).

The second purpose of this study was to examine the impact of the four different acculturation strategies (integration, assimilation, separation, marginalization) on the three different points of attachment and the three consumption behaviors for the respective points of attachment. For this purpose, the study included the three different identifications: (a) identification with ethnic player(s) (IEP), (b) identification with a popular sport in a native country (IPSNC), and (c) identification with a popular sport in the U.S. (the host country in this study) (IPSHC); and the three different sport consumption behaviors: (a) consumption for a team with an ethnic player (CTEP), (b) 
consumption for a popular sport in a native country (CPSNC), and (c) consumption for a popular sport in the host country (CPSHC).

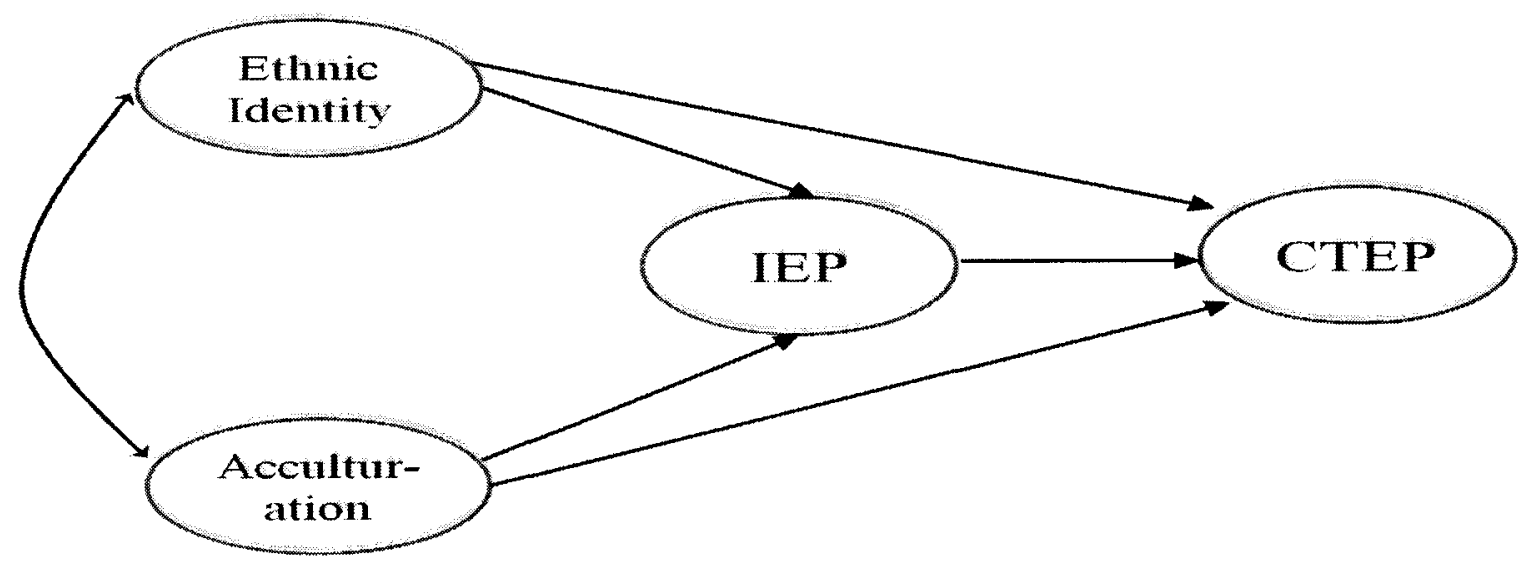

Figure 1-1. Ethnic Player Model. Note. EID = Identification with an Ethnic Player; and CTEP $=$ Consumption for a Team with an Ethnic Player.

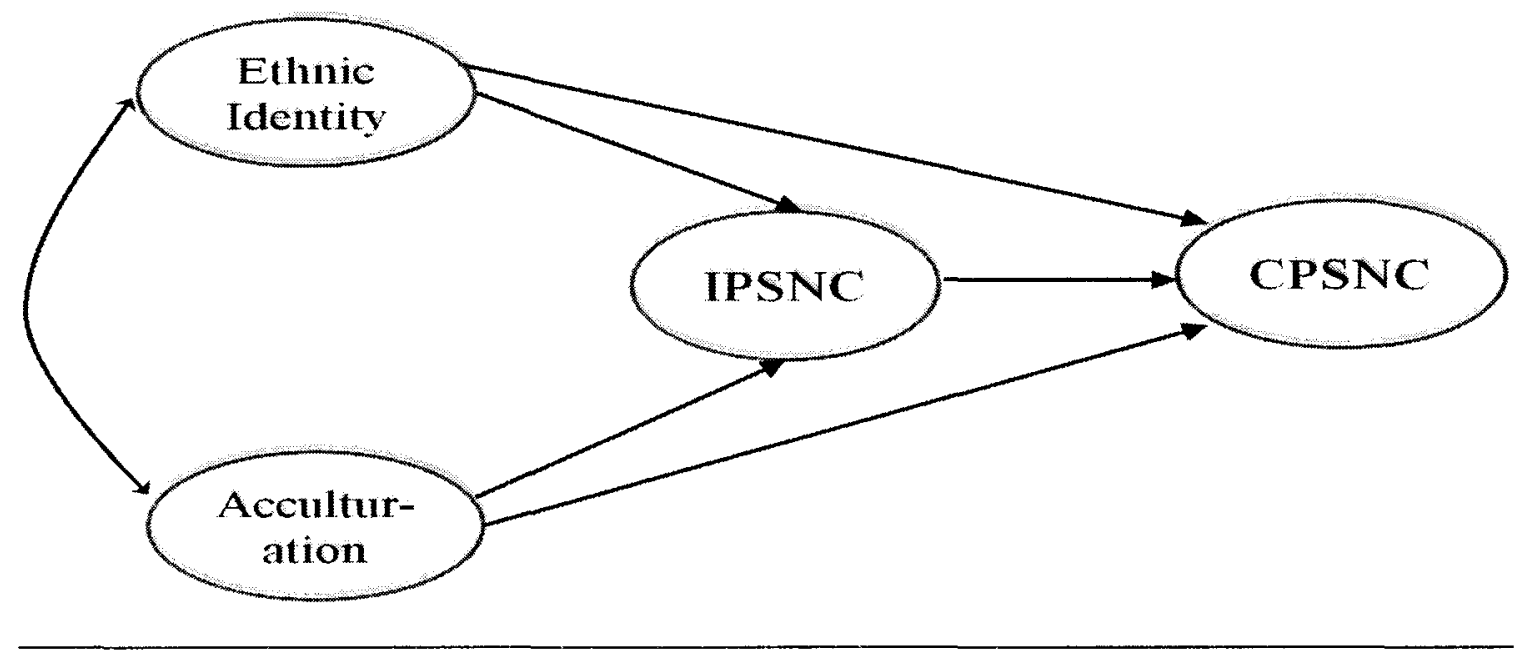

Figure 1-2. Native Sport Model. Note. IPSNC = Identification with a Popular Sport in a Native Country; and CPSNC $=$ Consumption for Native Sport $=$ Consumption for a popular sport in a Native Country. 


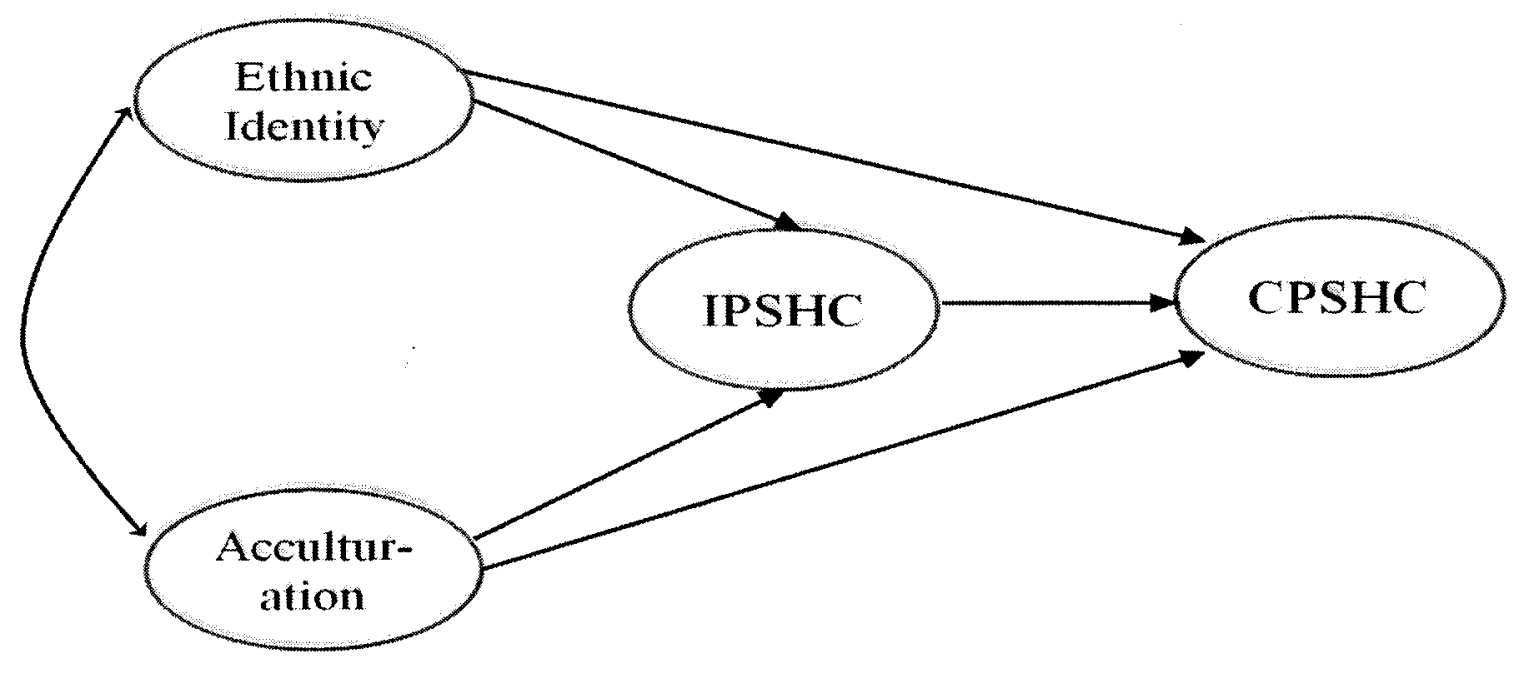

Figure 1-3. Host Sport Model. Note. IPSHC = Identification with a Popular Sport in a Host Country; and CPSHC = Consumption for a Popular Sport in a Host Country .

\section{Significance of the Study}

For a number of reasons (e.g., education, immigration, business), moving into a new society is a naturally and rapidly increasing phenomenon around the world, particularly in pluralistic societies such as the U.S. In the process of entering a new society, newcomers face situations in which they accept or reject consumption behaviors or practices different from the ones of their country of origin (Kaufman-Scarborough, 2000). This would undoubtedly impact sport consumption behaviors among ethnic groups. Given their large and rapidly growing economic power and unique sociodemographic characteristics, Asians constitute an important next market segment for sport marketers. However, Asian populations' sport consumption levels in the U.S. are still low (Spanberg, 2003). In order to enhance such an underdeveloped market segment, therefore, there is a need for research to examine the sport consumption behaviors of Asian population in the U.S. 
The literature to date suggests that one of the main problematic issues marketers face is a lack of understanding cultural and ethnic differences among various subgroups of Asian Americans (Clarke III \& Mannion, 2006; Kaufman-Scarborough, 2000; Ko et al., 2008). This problem may lead sport organizations to dismiss important subgroups of the Asian population possessing high levels of economic power, including people from China, Korea, Japan, and Taiwan. Given the combined importance of economic power and the relatively developed sport industries in these four countries, this study focuses on sport consumption behavior in the U.S. among Asians from China, Korea, Japan, and Taiwan.

The literature shows it is imperative for sport marketers to take cultural issues into account when attempting to develop marketing and promotional strategies (Armstrong, 2002b; Pons et al., 2001). Yet only three studies sought to examine the effects of ethnic identity and acculturation on identification with sports and sport consumption behavior (Harrole \& James, 2007; Pons et al., 2001; Shin, 2010), and the results of the three studies were inconsistent. Specifically, Pons et al. found that acculturation and ethnic identity simultaneously affected ethnic groups' sport consumption behavior and identification. In contrast, Harrole and Trail (2007) found that acculturation and ethnic identity had little influence on identification with various sports. Further, the two studies (Harrole \& James, 2007; Pons et al., 2001) only examined one component of points of attachment (identification with sports) rather than identification with athletes, which is an important point of attachment resulting in enhancing of fan-athlete identification for the Asian population (Ko et al., 2008; Min \& Kim, 2009). As mentioned previously, the 
current study examined sport consumption behavior models based on the three points of attachment (IEP, IPSNC, and IPSHC), ethnic identity, and acculturation.

Results of the current study could help sport marketers better understand Asian sport consumers in the U.S. It is particularly important to professional sports teams located in big cities such as New York, Los Angeles, San Francisco, Houston, Dallas, and Chicago where many Asians reside. While recruiting high-profile Asian players, Asians are more likely to identify with the athletes and ultimately attend and watch a team's event at which the Asian players appear. Indeed, several professional franchises have succeeded in attracting the Asian population after recruiting Asian athletes. Examples of these include Chan Ho Park (Korea), Yao Ming (China), Ichiro Suzuki (Japan), and Chen-Ming Wang (Taiwan).

When sport marketers develop marketing and promotional strategies for the Asian population in the U.S., they should provide promotional messages translated into the different languages of the targeted niche markets (Fattah, 2002; Morton, 1997). For example, the Houston Rockets have extended their marketing efforts to the Chinese community in part by providing a Chinese-version website, employing Chinese-speaking staff, and broadcasting a weekly Chinese-speaking radio program (Clarke III \& Mannion, 2006; Luo, 2003; Wang, 2009)

Furthermore, the results from research on the relationships among ethnic identity, acculturation, and identification with sports could help both sport practitioners and researchers in understanding the extent to which ethnic groups prefer to consume popular sports in a native country or those in a host country. Particularly, the results can be significantly important in understanding how ethnic groups consume the popular sports in 
a native country through the Internet, considering that consumption for the popular sports in a native country cannot occur by direct consumption forms (e.g., attendance).

Finally, four different acculturation strategies (integration, assimilation, separation, marginalization) would play critical roles in developing marketing and promotional strategies. Testing the strategies may provide insights in developing a new market segment tool for various ethnic minority groups.

\section{Research Questions}

To accomplish the two purposes mentioned above, this study contained four primary Research Questions. Research Questions 1, 2, and 3 were developed for the first purpose of the study (developing and testing Sport Consumption Models for the Asian population), while Research Question 4 was developed for the second purpose of the study (examining the impact of acculturation strategies on sport fan identifications and consumption behaviors). The first three Research Questions for the first purpose were as follows:

1. What is the relationship among ethnic identity, acculturation, identification with an ethnic player, and consumption behavior for a team with the ethnic player?

2. What is the relationship among ethnic identity, acculturation, identification with a popular sport in a native country, and consumption behavior for the sport?

3. What is the relationship among ethnic identity, acculturation, identification with a popular sport in the U.S., and consumption behavior for the sport?

The fourth Research Question for the second purpose and its three sub-questions are listed below: 
4. What are the relationships of four acculturation strategies (integration, assimilation, separation, marginalization) on each of the three different targets of identification and consumption behavior for the respective identification, while controlling for the effect of length of residence in the U.S.?

4-1: Are there significant differences in identification with an ethnic player (IEP) and consumption behavior for a team with the ethnic player (CTEP) among four acculturation strategies regardless of length of residence in the U.S.?

4-2: Are there significant differences in identification with a popular sport in a native country (IPSNC) and consumption behavior for the sport (CPSNC) among four acculturation strategies regardless of length of residence in the U.S.?

4-3: Are there significant differences in identification with a popular sport in the U.S. (IPSHC) and consumption behavior for the sport (CPSHC) among four acculturation strategies regardless of length of residence in the U.S.?

\section{Delimitations}

The current study contained the following delimitations:

1. This study included only Asians populations who came from four countries (China, Japan, Korea, and Taiwan). Thus, the results of the current study might not be generalizable to other Asian populations living in the U.S. In addition, since data collection occurred in cities in which Asian athletes are currently playing or recently played, the findings might not be generalizable to people living other locations of the U.S.

2. This study focused on only two points of attachment (i.e., sport and athlete). Thus, the findings might not be generalizable to other points of attachment (e.g., coach, team). 
3. This study has solely focused on ethnic players in professional team sports rather than those in professional individual sports (e.g., PGA, LPGA).

\section{Limitations}

The current study contained the following limitations:

1. This study aimed to examine the effects of acculturation and ethnic identity on fan identification and consumption behavior. Participants' level of acculturation and ethnicity may be influenced by other factors (e.g., gender, length of residence in the U.S./generational status, general level of sport fandom, living location).

2. The researcher recruited potential participants from Asian cultural/community centers and Asian student associations in universities. Thus, it is possible that these people are already highly involved and actively participate in their cultures of origin. This may make them different from people with the same cultural background who are not associated with Asian student centers or Asian cultural/community centers.

3. Although the instruments used for the current study have demonstrated reliability and validity, they might still be unable to completely control other variables beyond and above the underlying theoretical structure.

4. Questions regarding popular sports in both native and host countries were already determined before distributing the questionnaire. It is possible that participants' perceptions of the popular sports might be different than those provided by the research.

5. Participants' level of identification and consumption behavior might be influenced by other extraneous variables (e.g., sport fan motivation, athletic performance, athlete transfer). 
6. Participants who fail to complete or return the survey questionnaire may differ from participants who completed and returned the study and therefore may be likely to influence the results of the current study.

7. To categorize four acculturation strategies (integration, assimilation, separation, marginalization), the median split was employed. Even though the median split provided relatively balanced sample size among the four acculturation groups, artificial categorization using the median split may lead to conflicting results the previous literature. Therefore, extra caution should be taken when interpreting the results regarding acculturation strategies.

\section{Definitions of Terms}

Acculturation - "A multidimensional process of change that occurs when individuals of differing cultural groups come into continuous contact" (Stephenson, 2000, p. 77). Asian Population in the U.S. - A person having origins in any of the original peoples of Far East, Southeast Asia, or the Indian subcontinent including, for example, Cambodia, China, India, Japan, Korea, Malaysia, Pakistan, the Philippine Islands, Thailand, and Vietnam (U.S. Census Bureau, 2011). In this study, Asian population refers to those individuals living in the U.S. either permanently or temporarily.

Culture of Origin - One's original cultural background based upon ethnicity, nationality, or country of birth one's parents or ancestors before their arrival in the U.S. In this study, 'culture of origin,' 'original culture,' or 'native country' are regard as the same meaning.

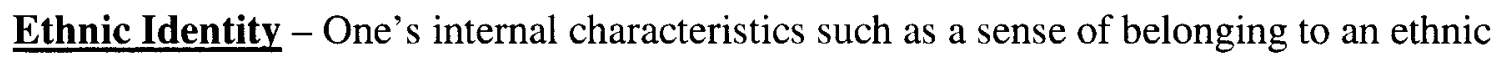
group and the attitudes, perceptions, and feelings related to that membership (Phinney, 1996; Phinney, Cantu, \& Kurtz, 1997). 
Ethnic Minority Groups - a group that has different national or cultural traditions/backgrounds from those of the majority population. In the current study, ethnic minority groups include African Americans, people of Hispanic, Asian-Pacific, and Native-Indian ancestry and descent (Armstrong, 2008).

Fan Identification - A psychological connection or an orientation that individuals feel and attach toward sport-related objects, such as a team, player, coach, university, or community (Dietz-Uhler \& Lanter, 2008; Robinson, Trail, \& Kwon, 2004; Trail, Anderson, \& Fink, 2000).

Hispanic or Latino - A person of Cuban, Mexican, Puerto Rican, South or Central American, or other Spanish culture or origin regardless of race (U.S. Census Bureau, 2010).

Host Society - A new physical/cultural environment or place where newcomers (e.g., immigrants, sojourners, refugees) live with a predominant group's values, behaviors, and norms. In this study, 'host society,' 'host country,' or 'dominant culture/society' are considered the same.

Sport Consumption - The personal and social process through which individuals consume sport products/services through attendance at sporting events, participation in sport activities, or consumption of sports via the electronic and print media including TV, newspaper, sport magazine, the Internet, and radio. 


\section{CHAPTER II}

\section{LITERATURE REVIEW}

There were two purposes for the current study. The first purpose was to develop and test Sport Consumption Models for the Asian population living in the U.S. based on ethnic identity and acculturation. For this purpose, this study explored the relationships among ethnic identity, acculturation, three points of attachment [identification with an ethnic player (IEP), identification with a popular sport in a native country (IPSNC), and identification with a popular sport in a host country (IPSHC)], and three sport consumption behaviors [consumption for a team with an ethnic player (CTEP), consumption for a popular sport in a native country (CPSNC), and consumption for a popular sport in a host country (CPSHC). The three proposed models for this purpose were presented in Figures 1-1 (Ethnic Player Model), 1-2 (Native Sport Model), and 1-3 (Host Sport Model).

The second purpose of this study was to examine the impact of the four different acculturation strategies (integration, assimilation, separation, marginalization) on the three different points of attachment and the three consumption behaviors for the respective points of attachment. For this purpose, the study included the three different identifications: (a) identification with ethnic player(s) (IEP), (b) identification with a popular sport in a native country (IPSNC), and (c) identification with a popular sport in the U.S. (the host country in this study) (IPSHC); and the three different sport 
consumption behaviors: (a) consumption for a team with an ethnic player (CTEP), (b) consumption for a popular sport in a native country (CPSNC), and (c) consumption for a popular sport in the host country (CPSHC).

In this chapter, the researcher examined seven main areas: attributes of the U.S. Asian population, ethnic identity, acculturation, consumption behavior among ethnic minority groups, sport consumption behavior among ethnic minority groups, ethnic identity and acculturation in a sport context, and identification in a sport setting.

More specifically, the literature review began with the unique attributes of the U.S. Asian population and then covered research and theories pertinent to ethnic identity. Following the review of ethnic identity were theories and research pertinent to acculturation. The next topic covered in the literature review was a summary of studies examining consumption behaviors/patterns among ethnic minority groups, which enable readers to understand how ethnic minority groups consume differently in a dominant culture, and then covered sport consumption behavior among ethnic minority groups and specific research dealing with ethnic identity and acculturation in a sport context. Lastly, the researcher reviewed sport fan identification and its outcomes, which include various sport fan behaviors.

\section{Attributes of the Asian Population in the U.S.}

Although ethnic minority groups moved to the U.S. more than 250 years ago, a significant and unanticipated increase of immigrants from diverse ethnic/geographic groups, including Asia, Mexico, South America, and other Western nations began in the late 1960s shortly after the passage of The Immigration and Nationality Act of 1965 (Le, 2010b). Due in part to The Act, the American population is becoming more diversified. 
In the diversified population, the three largest ethnic groups are African Americans, Hispanics, and Asians. Of the three minority groups, the media has particularly described Asian Americans as a 'super minority,' 'the fastest growing minority,' and 'the next hot market' (Lee \& Um, 1992) due to their unique characteristics in several aspects.

The 2010 total population of Asian descent in the U.S. who said they are Asian or Asian-related ethnicities was 17.3 million (U.S. Census Bureau, 2011), and the projected percentage increase of Asians in the U.S. between 2000 and 2050 is 213\%, which is projected to be more than five times the increase of U.S. total population (49\%) over the same period of time (Le, 2010a). For the geographic distribution of the Asian population, more than $60 \%$ of Asian populations live in ten states: California, New York, Hawaii, Texas, New Jersey, Illinois, Washington, Florida, Virginia, and Massachusetts (U.S. Census Bureau, 2007). More specifically, the top three metropolitan areas with the largest proportion of Asian populations are the Los Angeles Area (1.87 million in 2007), the New York-Northern New Jersey-Long Island Area (1.78 million in 2007), and the San Francisco Bay Area (979,000 in 2007) (U.S. Census Bureau, 2008). Due to the geographically concentrated characteristic of the Asian population, some professional sport franchises where the high proportion of Asian populations are living have begun to recruit popular and high-profile Asian athletes, such as Chan-Ho Park (LA Dodgers), Ichiro Suzuki (Seattle Mariners), Hideki Matsui (New York Yankees), Yao Ming (Houston Rockets), and so on. These remarkable demographic figures clearly show that the Asian population is a powerful ethnic group in the U.S. consumer market. When it comes to Asian Americans' economic impact, the Selig Center for Economic Growth reported that the nation's Asian purchasing power is anticipated to 
increase over 600\% from \$116 billion in 1990 to $\$ 697$ billion in 2014 (Humphreys, 2009). In particular, the buying power of Asian Americans will grow faster than that of any other ethnic minority group except for the Hispanics (Humphreys). Furthermore, the median household income for Asians in the U.S. was the highest among all ethnic groups in 2005 (\$64,238; Lee, 2010a).

In terms of education, Asians are typically described as highly education-oriented people. As a matter of fact, $49 \%$ of Asians aged 25 and older have a bachelor's degree or higher level of education (e.g., Master's, Ph.D., M.D. or J.D.) (Lee, 2010a). Approximately $20 \%$ of Asians aged 25 and older hold advanced degrees, while only $10 \%$ of their American counterparts have similarly high levels of education.

Due in part to Asians' typically high levels of formal education, however, some negative stereotypes exist. For example, some people believe that Asians are not interested in participating in sports because Asians are often seen as intelligent, mathematically skilled, and technically competent instead of athletically inclined. While it is true, Asian Americans place great value on education rather than athletics or sports, many athletes of Asian descent in recent years are showing outstanding performance in international sporting events as well as in U.S. professional sport leagues. The athletic successes of Asian players are altering the negative images of Asians with regard to athletics and sports and have significantly influenced involvement in sports among Asian populations and eventually led to their participation in other aspects of U.S. society (Lapchick, 2008).

Considering the Asian population is one of the fastest growing segments in the U.S. in terms of growth rate and has considerable economic potential in terms of purchasing 
power and household income, marketers in various industries should be interested in targeting Asian Americans to generate more profits. Despite Asians' potential in the U.S. market, one primary stereotype that persists is that all Asian Americans are the same (Asian-Nation, n. d.). In other words, many people - especially non-Asians - are generally unable to distinguish different Asian ethnic groups (e.g., Korean Americans from Chinese Americans). In reality, applying generalizations about one or a few Asian Americans to the whole Asian American population can become a problem in that Asian populations consist of more than 30 different ethnic subgroups spread across the U.S, each of which has its own culture (Kaufman-Scarborough, 2000). To that end, it would be highly advantageous for marketers to define segments to strengthen their efforts in reaching these diverse ethnic groups.

Despite its diverse subgroups, marketing and advertising agencies have typically divided Asian populations into three segments to strengthen their efforts in reaching the diverse Asian subgroups based on their regional and cultural closeness: (a) Northeast Asians (e.g., Chinese, Korean, Japanese, Taiwanese, Vietnamese), (b) Southeast Asians (e.g., Filipino, Malaysian, Indonesian, Cambodian), and (c) South Asians (e.g., Indian, Pakistani, Bangladeshi) (Magazine Publishers of America, 2004). From a marketer's standpoint, such group market segmentation of Asian population may be more proper than individually dividing a variety of Asian subgroups due to its cost-effectiveness in terms of management of various subgroups.

Of more than 30 different ethnic subgroups, people from the four specific countries, China, Korea, Japan, and Taiwan, have relative high levels of economic power. For the sport industry, currently popular professional sports in these four countries are also 
similar to those in the U.S. For example, baseball and basketball represent popular sports in all four countries in terms of both participants and spectators, although baseball in China is relatively unpopular. Japan has Nippon Professional Baseball (NPB) with its Central and Pacific Leagues, Korea has the Korean Baseball Organization (KBO), and the Taiwan Major League of Professional Baseball (TML). Furthermore, almost all Asian athletes playing in the four major professional sports leagues (i.e., MLB, NBA, NFL, NHL) in North America also came from the four countries. By marketing the Asian athletes to Asians living in the U.S. from the four subgroups, professional sports teams and leagues in the U.S. may effectively increase their revenues. Lastly, people from these four countries are more likely to consume sports because the four countries have relatively well developed sport industries compared to other Asian countries. Thus, the current study focused on these four ethnic subgroups located in the region of Northeast Asia.

In order to better understand sport consumption behavior of the four specific ethnic groups, it is essential to review ethnic identity which may influence their sport consumption.

\section{Ethnic Identity}

From an historical perspective, ethnic identity has emerged as an important social issue since the U.S. Civil Rights movement of the 1960s (Laosa, 1984) and began to receive increasing attention due to the variation of demographics not only in the United States but also in other countries throughout the world. Particularly in the U.S., ethnic identity became a more salient issue for both members of ethnic minority groups and 
those of the dominant society because of the rapid growth of ethnic minority groups (e.g., Hispanic and Asian American) (Phinney, 1992).

Before defining the term ethnic identity, it is important to differentiate similar terms (e.g., , ethnicity, ethnic group, race) from ethnic identity. Although the concept of ethnicity itself is defined in various ways across disciplines, it is generally referred to as demographic characteristics, including common language, culture, or national origin (Quintana, 2007). Similarly, ethnic group refers to a set of individuals sharing distinctive characteristics above such as ancestry, culture, language, religion, or national origin (Phinney, Horenczyk, Liebkind, \& Vedder, 2001). Race refers to a set of individuals who are thought to share physical characteristics (e.g., skin tone) and biological descent. As such, while ethnicity has traditionally been defined with the basis of demographic characteristics, race is typically assumed to have a biological and physical basis (Quintana, 2007). In contrast to such obvious and superficial characteristics, ethnic identity has been conceptualized as one's internal characteristics, such as a sense of belonging to an ethnic group and the attitudes, perceptions, and feelings related to that membership (Phinney, 1996).

Even though ethnic identity often refers simply to one's self-label or group affiliation, the term generally involves the various aspects mentioned above, such as selfidentification, a sense of belonging and commitment to a group, a sense of values and attitudes toward one's ethnic group, and participation in cultural activities such as shared language, behaviors, friendship, religion, politics, and any combination of these (Persky \& Birman, 2005; Phinney, 1990, 1996; Phinney, Horenezyk, Libkind, \& Vedder, 2001). In other words, researchers believe that ethnic identity is a complex multidimensional 
concept, including perceptions, awareness, affects, and knowledge, among other aspects, about, particularly with regard to two or more cultures (Cuellar, Nyberg, Maldonado, \& Roberts, 1997). Although ethnic identity has been defined in numerous ways, it appears that ethnic identity refers to "an enduring, fundamental aspect of the self that includes a sense of membership in an ethnic group and the attitudes and feelings associated with that member [ship]" (Phinney, 1996, p. 922). The following section reviewed the research dealing with ethnic identity.

\section{Research on Ethnic Identity}

Phinney (1990) attempted to review a total of 62 empirical studies on ethnic identity in adolescents and adults. Her review gave great insight into understanding the concept of ethnic identity, its essential components, and its measurement and conceptualizations. Approximately half of the studies reviewed focused on White ethnic groups (e.g., Greek and Italian Americans or French Canadians) followed by Black groups. To identify what components were critical to assess ethnic identity in the past research, she grouped them into four categories: (a) self-identification (i.e., ethnic label), (b) sense of belonging, (c) attitudes, and (d) involvement in cultural activities (i.e., behaviors and practices relative to one's own group). Of those multidimensional components, different components have been chosen by researchers depending on different ethnic groups. For example, language and a variety of cultural activities were most widely used to assess ethnic identity of White ethnic groups and Hispanics, while attitudes were treated as a dominant component of ethnic identity for African Americans. In addition, she discussed the relationships among essential components of ethnic identity. Some studies examined the relationship between ethnic self-identification and 
ethnic behaviors, and others sought to investigate the relationships among various aspects such as pride, sense of affiliation, and ethnic self-identification. However, the relationships derived from various studies showed conflicting results because of the differences in defining and measuring components.

In the review on ethnic identity, Phinney (1990) insisted that the research on ethnic identity has varied depending on the group's relationships to the dominant culture. In other words, while some studies have only focused on a single group, others examined one's ethnic identity in relation to the identity of the dominant culture. The latter studies were divided into two models depending on whether or not the relationship between one's own ethnic identity and the identity of the dominant culture was independent: (a) bipolar model and (b) dual focus. In the bipolar model, ethnic identity is conceptualized as a continuum, from strong identification with one's own ethnic group to strong identification with the dominant culture. In this case, the relationship between the two identities (ethnic group vs. dominant group) is not independent, suggesting that one's strong ethnic identity might have a negative relationship with his/her identification with the dominant group. In contrast, the main point of dual focus is that the relationship between the two identities is independent, so reflecting that a strong ethnic identity does not necessarily imply a weak identification with the dominant culture. Phinney (1990) argued that many studies with dual focus were based on Berry's acculturation model with two orthogonal dimensions (Berry, 1990, 1992, 1997; Berry, Trimble, \& Olmedo, 1986) that will be discussed in a later section.

Another line of research that Phinney reviewed indicated ethnic identity can change over time, settings, and according to gender. Several studies sought to investigate 
differences in ethnic identity based upon the generation status of immigrants. For example, the longer immigrants live in the dominant culture, the weaker ethnic identity becomes. Some research examined how ethnic identity varied depending on environmental factors, such as 'where they live.' In addition, a few researchers investigated whether there is a difference in ethnic identity between males and females. Typically, these studies found that females were more likely to have higher levels of ethnic identity than males.

Phinney (1990) also discussed a model of developmental changes in ethnic identity with Erikson's (1968) ego identity model and Marcia's (1966) four identity statuses based upon the extent of exploration and commitment toward an identity. Individuals classified as diffuse have neither engaged in exploration nor made a commitment toward identity. Those who have made a commitment without exploration are classified as foreclosed. Those in the process of exploration without having made a commitment are in moratorium. Lastly, a strong commitment following a period of exploration is indicative of identity achievement. The fundamental assumption of the model is that individuals are more likely to select an ethnic identity as they become older.

Even though discussing theoretical bases of research on ethnic identity was not the goal of her review, Phinney (1990) described several theoretical frameworks used in ethnic identity: social identity theory, acculturation and assimilation, and identity formation. However, she argued that it was difficult to build an integrated theory for ethnic identity at the time of the study due to the lack of strong empirical data on ethnic identity. 
Finally, Phinney suggested two important issues for future research on ethnic identity in order to resolve the inconsistencies of the results and structure that were evident in prior research. These included agreement on a common definition to be used by researchers and development of a valid and uniform measurement that can be used across groups.

In response to the recommendations above, Phinney (1992) developed a measure of ethnic identity that can be used with diverse ethnic groups and can be compared across findings from diverse groups. This measurement has been called the Multigroup Ethnic Identity Measure (MEIM). Based on the existing literature on ethnic identity, the MEIM was composed of three components representing ethnic identity: positive ethnic attitudes and sense of belonging ( 5 items); ethnic identity achievement, including both exploration and resolution of identity issues ( 7 items); and ethnic behaviors or practices ( 2 items). In addition to these items, the author included six items to assess orientation and attitudes toward other groups. Demographic information, such as gender, age, and parental education, was also included in the questionnaire.

In Phinney's initial research on the MEIM, the questionnaire was completed by both high school students $(n=417)$ and college students $(n=136)$ from an ethnically diverse urban high school and university respectively.

The reliability of the 14-item MEIM was .81 for the high school sample and .90 for the college sample based on Cronbach's alpha. The findings of her study demonstrated that the MEIM was a reliable measure for assessing ethnic identity for a variety of ethnic groups, regardless of the unique characteristics of the ethnic group. With the findings, Phinney (1992) concluded that although numerous studies on ethnic identity focused on 
unique aspects of one's own ethnic group, ethnic identity can be conceptualized and measured as a general phenomenon with commonalities across diverse ethnic groups.

Throughout the years, the MEIM has been continuously revised by several researchers (Phinney \& Ong, 2007; Roberts et al., 1999) and could best be thought of as consisting of two factors, exploration and commitment. The exploration factor consists of three items dealing with efforts to learn more about one's own ethnic group and involvement in ethnic cultural practices, while the commitment factor included three items, describing both a positive affirmation of one's group and a clear sense of commitment (Phinney \& Ong, 2007).

Phinney, Horenezyk, Liebkind, and Vedder (2001) reviewed theory and literature on ethnic identity and national identity (i.e., identity with a dominant society) using the concept of acculturation to better understand their interrelationship. The authors also sought to explore how these identities played a role in the psychological well-being of immigrants. In their study, the authors utilized the findings from a study of immigrant youth in four immigrant receiving countries: the United States, Israel, Finland, and the Netherlands.

According to Phinney and her colleagues (2001), the relationship between ethnic identity and national identity can be understood by a two-dimensional model (or bidimensional model of acculturation) (Berry, 1990, 1997). The main point of Berry's two dimensional model of acculturation is that immigrants' acculturation strategies can vary depending on two dominant aspects of acculturation (preservation of one's heritage culture and adaptation to the dominant society), which are assumed to be independent of each other. Using the two dimensional model of acculturation, the authors suggested that 
immigrants could belong to one of four possible identity categories: (a) integrated identity (high ethnic identity and high national identity of host country), (b) separated identity (high ethnic identity and low national identity, (c) assimilated identity (low ethnic identity and high national identity), and (d) marginalized identity (low ethnic identity and low national identity).

The authors explained the strength and the relationships of the two identities with their unpublished study on immigrant adolescents. The results of the unpublished work revealed that ethnic identity and national identity (identity with a host country) were unrelated. However, the relationships between the two identities were remarkably different among countries and among ethnic groups within countries. Phinney et al. (2001) argued that these differences might be due to contextual factors, such as host country immigration policies. In other words, for example, immigrants living in a host country with integration immigration policies respecting cultural diversity are more likely to select integrated identity (high ethnic identity and high national identity) among the four identity categories.

Phinney et al. (2001) also discussed how ethnic identity affects immigrants' psychological well-being and suggested that a strong and secure ethnic identity is positively related to immigrants' psychological well-being. In addition, they argued that of the four identities, integrated identity (a strong ethnic identity with both their ethnic group and the dominant society) is expected to be the most conducive to immigrants' well-being. Finally, Phinney et al. (2001) suggested that the relationship between ethnic identity and national identity can be moderated by several factors, such as gender, the length of residency in the dominant society, and immigrant generation. 
Cuellar and his colleagues (1997) sought to examine what relationship existed between ethnic identity and acculturation (particularly behavioral acculturation) among members of the Mexican origin population. In addition, they attempted to see if there were significant differences in ethnic identity based upon various acculturative types and generational status. The sample size was 1,367 students, $87 \%$ of whom were of Mexican origin. Ethnic identity was measured by the Multigroup Ethnic Identity Measure (MEIM) that consisted of three subscales: (a) affirmation and belonging, (b) ethnic identity achievement, and (c) ethnic behaviors. Acculturation was assessed using the Acculturation Scale for Mexican American-II (ARSMA-II). ARSMA-II has two distinctive subscales, a Mexican Orientation Subscale (MOS), and the other is an Anglo Orientation Subscale (AOS). Using a unidirectional model of acculturation, Cuellar et al. (1997) divided participants into five levels, with level 1 representing least acculturated and level 5 representing most acculturated. Furthermore, based upon cutting scores from the two subscales (MOS and AOS) (see Cuellar, Arnold, \& Maldonado, 1995), participants were classified into the following four acculturative types: traditional Mexican group, high bicultural group, low bicultural group, and assimilated group.

The findings showed that ethnic identity was negatively correlated with acculturation $(r=-.32)$, indicating that as one's sense of ethnic identity among the Mexican origin population increased, acculturation into American culture decreased. Each of the three subscales of ethnic identity was negatively correlated with acculturation. In terms of group differences, individuals in the less acculturated, traditional Mexican group, and first generation immigrants revealed strong levels of 
ethnic identity. Lastly, people in the high bicultural group tended to have higher scores on ethnic identity than those in the low bicultural group.

Originally, there were four acculturative types/modes/patterns according to Berry $(1990,1997)$. One of them was integration (high orientation into both a host culture and a culture of origin). By distinguishing this type of acculturation into two different acculturative types (high bicultural and low bicultural), this study demonstrated the complexity of the acculturation process.

\section{Theories Relative to Ethnic Identity}

In prior research, the two major theories focused on ethnic identity were social identity theory and identity formation theory. These two theories are essential in understanding the members of any ethnic group.

Social identity theory. One conceptual framework often used to study ethnic identity is social identity theory (Tajfel, 1982). According to Tafjel and Turner (1986), social groups have been defined "as a collection of individuals who perceive themselves to be members of the same social category, share some emotional involvement in this common definition of themselves, and achieve some degree of social consensus about the evaluation of their group and of their membership in it" (p. 15). The social groups to which people belong help define who they are and thus facilitate a way for group members to create their place in society (Tajfel \& Turner, 1986). The key concept of social identity theory is that individuals in a society use group memberships to define themselves. Tajfel (1981) depicted social identity "as that part of an individual's selfconcept which derives from his knowledge of his membership of a social group together with the value and emotional significance attached to membership" (p. 255). 
According to Tajfel (1981), social identity theory can be described several ways with respect to group membership: (a) individuals tend to seek out or maintain memberships in social groups that provide a positive aspect to their self-esteem or self image; (b) an individual does not want to maintain a group membership if she/he is not satisfied with a need for positive identity from the group membership; (c) if an individual cannot leave group membership, she/he will adapt, justify a lower status, or simply accept the situation; and (d) all groups in society live among other groups. From these stipulations, individuals are typically motivated by the desire to maintain and develop a favorable self-concept or self-image (i.e., self-esteem); thus they attempt to join groups that a positively impact their self esteem (Tajfel, 1982).

One of the important tenets of social identity theory is the classification of ingroups and out-groups (Hogg \& Abrams, 1990). The term in-group refers to individuals who belong to a salient group, whereas the term out-group refers to those who do not belong to the salient group. For instance, if an individual identifies as a White American, all other White Americans would be regarded as in-group members when that group membership is deemed important, while all other ethnic groups (African Americans, Latino Americans, Asian Americans, etc.) would be regarded as out-group members. As mentioned above, social identity theory posits that individuals seek to join groups that have a positively impact their self-concept, thus boosting their self-esteem. In order to increase self-esteem, individuals make comparisons based upon group memberships and sometimes classify members of an out-group as less important than those of an in-group. For example, a White American may feel better about his/her own group members as an in-group and may provide special favors to other White Americans 
that would not be offered to members of the other groups, such as Asian American, Latino Americans and African Americans (out-group members). According to Liebkind (1992), immigrants in a new society are often viewed in negative or derogatory ways by the dominant society and may take a variety of positions in the face of derogation and devaluation of their group. In situations where the immigrant group is not viewed positively by the dominant society, individuals within the immigrant group may work hard to develop pride with their group and emphasize the uniqueness of their ethnic group (Phinney et al., 2001).

Within the framework of social identity theory, ethnic identity has been regarded as one portion of one's general social identity that draws from membership in the individual's ethnic group, and that brings the value and emotional worth that attaches to that membership (Tajfel, 1981). In addition, Phinney (1990) defined ethnic identity as an individual's retention and attainment of cultural uniqueness that are integrated into the self-concept, which develops in the background of the individual being a member of a minority ethnic group within the dominant society. The researchers had a consistent argument that social identity theory was employed to look at the complexities deriving from negotiating two cultures; therefore, immigrants face conflicts with behaviors, values, and attitudes between their culture of origin and the dominant culture.

Identity formation theory. The identity formation theory has its roots in the ego identity model of Erikson (1968). According to Erikson, identity is not something individuals automatically possess, but something that changes over time. From the perspective of identity formation theory, an individual's achieved identity results from two processes, exploration and commitment, and individuals will have a single and 
unique identification which encompasses all prior significant identifications during the last stages of adolescence (Erikson, 1968; Phinney, 1990). Marcia’s (1980) empirical work on personal identity, derived from Erikson's model, suggested four identity statuses depending on the degree of exploration and commitment: diffuse, foreclosed, moratorium, and achieved identity. Individuals may engage in neither exploration nor commitment, indicating a diffuse status. If individuals have made a commitment without exploration, they are in foreclosed status. If individuals are in the process of exploration without making a commitment, they are in moratorium status. Finally, if individuals have explored key identity issues and made commitments, they are in an achieved identity (Marcia, 1980; Phiney, 1990; Phinney \& Ong, 2007). However, Marcia's work only dealt with personal identity, involving areas of choice in the formation of an identity, such as political and occupational identities, and did not examine ethnic identity. To fill this gap, a three-stage model of ethnic identity development based om Marcia's four identity statuses was proposed by Phinney (1989): unexamined ethnic identity, ethnic identity search, and achieved ethnic identity.

Although individuals cannot choose ethnicity as it is assigned to them by others according to their ethnic background, ethnic identity has a similar characteristic with identity formation theory in which individuals explore and make commitments to their ethnic groups. According to Phinney and Ong (2007), ethnic identity “is assumed to undergo a major developmental change in adolescence and young adulthood, through the joint processes of exploration and commitment (Phinney \& Ong, 2007, p. 275) and therefore can be explained by the two processes. For example, we expect that individuals shift from ethnic identity diffuse (lack of distinct identity) to either moratorium 
(exploration without making a commitment), foreclosure (a commitment without exploration), or to an achieved ethnic identity. In spite of the fact that the process of ethnic identity formation usually occurs in adolescence or childhood, it may be true that the process can be continued throughout adulthood (Phinney, 2006) when individuals have acquired a relatively stable and secure sense of ethnic identity.

In addition to social identity theory and identity formation theory, which were mentioned above, acculturation has been regarded as a critical framework for the study of ethnic minority groups, along with ethnic identity. According to Cuellar et al. (1997, p. 536), "ethnic identity comprises an important overall referent of psychological acculturation." Therefore, it is important to understand the concept of acculturation in relation to ethnic identity.

\section{Acculturation}

Before examining the concept of acculturation, we need to clarify the differences between the constructs of acculturation and ethnic identity because a review of the literature showed that the two terms are frequently used interchangeably (Nguyen, Messes, \& Stollak, 1999), and the distinction between the two terms is still ambiguous (Liebkind, 2001; Phinney, 1990). To prevent this confusion among readers, Phinney (2003) clarified that acculturation is viewed as a broader construct, including an array of attitudes, behaviors, and values that change with contact between two or more different cultures. Ethnic identity is viewed as the most important part of the acculturation process that focuses on the subject sense of belonging to an ethnic group. Based on theoretical models on acculturation, ethnic identity tends to change as a function of acculturation processes. 


\section{Historical Perspectives of Acculturation}

In general, acculturation is seen as a process by which the behaviors and attitudes of newcomers (e.g., immigrants, sojourners) from diverse cultures are altered as a result of contact with the dominant or host society. However, since Robert Park from the University of Chicago first started to study on the concept of the melting pot in 1914, acculturation has been redefined by many scholars (Padilla \& Perez, 2003). Based on the theoretical framework, Park created a three-stage model consisting of (a) contact, (b) accommodation, and (c) assimilation (Persons, 1987). According to this model, as contact occurs between individuals from different cultures, they start to acclimate to each other followed by a process of cultural assimilation to the dominant society. Park's three-stage model served as momentum to stimulate thinking about how newcomers adjust to the dominant society (Padilla \& Perez, 2003).

After Park's three-stage model, a group of anthropologists, Redfield, Linton, and Herskovits (1936) greatly contributed to defining and theorizing about the construct of acculturation. The classical definition of acculturation provided by these anthropologists is that "acculturation comprehends those phenomena which result when groups of individuals having different cultures come into continuous first-hand contact with subsequent changes in the original patterns of either or both groups" (Redfield et al., 1936, p. 149). This definition clearly pointed out that "continuous first-hand contact" between individuals from different cultures is the critical component of acculturation. Another important aspect of this definition is that acculturation is a neutral term; that is, alterations in cultural patterns may take place in "either or both groups" (p. 149). In reality, however, it seems the acculturation process is more likely to occur in individuals 
from the original culture (e.g., Asian immigrants) than those from the dominant culture (e.g., White Americans) (Berry, 1990).

Almost 20 years later, the Social Science Research Council (1954) defined the process of acculturation in detail by adding psychological components including individuals' value systems, developmental sequences, and personal factors. The definition is as follows:

Acculturative change may be the consequence of direct cultural transmission; it may be derived from noncultural causes, such as ecological or demographic modifications induced by an impinging culture; it may be delayed as with internal adjustments following upon the acceptance of alien traits or patterns; or it may be a reactive adaption of traditional modes of life. Its dynamics can be seen as the selective adaption of value systems, the processes of integration and differentiation, the generation of developmental sequences, and the operation of role determinants and personality factors (Social Science Research Council, 1954, p. 974).

An essential component of this definition is that assimilation into the dominant culture is not the only kind or way of acculturation. That is, it allows individuals to select what elements of their original culture they want to retain or those of the host culture they want to integrate (Padilla \& Perez, 2003). Taking this perspective as an initial point, Berry $(1980,1990,1992,1997)$ first distinguished that acculturation can take place in various ways, including assimilation, integration, separation/segregation, and marginalization/deculturation.

\section{Two Levels of Acculturation (Group vs. Individual)}


The term acculturation generally describes the changes an individual experiences once he/she has direct contact with a host society. This is termed psychological acculturation (or individual level of acculturation) by Graves (1967) (as cited in Berry, 1997). Acculturation occurs on two levels - group and individual. The group level acculturation refers to changes occurring in political organizations, the economy, and the social structure, while the individual level acculturation refers to an individual's psychological changes, such as behavior, values, and attitudes (Berry, 1990, 1997). Even though an individual's acculturation process is affected by the group level of acculturation, the two levels of acculturation do not necessarily evolve in the same way. For example, an individual may have a high level of acculturation, whereas the group he/she belongs to may be lowly acculturated or vice versa. According to Berry (1997), the main reason to distinguish between the two levels of acculturation is "because not all individuals participate to the same extent in the general acculturation being experienced by their group. While the general changes may be profound in the group, individuals are known to vary greatly in the degree to which they participate in these community changes" (Berry, 1997, p. 7). Thus, the focus of the current study is on each individual level of acculturation.

\section{Theoretical Models of Acculturation}

According to a thorough review of literature on acculturation, particularly in psychology and cross-cultural research, the acculturation process has evolved from a unidimensional (also called unidirectional and unilinear or bipolar) model to a bidimensional (also called bidirectional and two-dimensional) model. Therefore, we will discuss each of the two approaches on acculturation in the following sections. 
Unidimensional model (Bipolar model). The unidimensional model is grounded in the assimilationist perspective. From this point of view, it is assumed that individuals from non-dominant cultures will adapt values and customs of the dominant culture (i.e., the host culture) over time. According to this model, adaptation to the dominant culture is seen as necessarily accompanied by a weakening of ties to one's culture of origin so that an acculturated individual almost gives up his or her culture of origin (Nguyen, Messe, \& Stollak, 1999). Thus, the culture of origin and the dominant culture are negatively correlated with each other. This model can be also described by a single continuum (see Figure 2); that is, newcomers move from one end of the continuum, reflecting high participation and involvement with the behaviors and values of their original culture, to the other end of the continuum, reflecting high participation and involvement with those of the dominant culture (Berry, 1997). In this single continuum, the midpoint between the two ends represents biculturalism, indicating that newcomers have high participation and involvement with characteristics of both their original culture and dominant culture (Keefe \& Padilla, 1987; Nguyen \& von Eye, 2002).

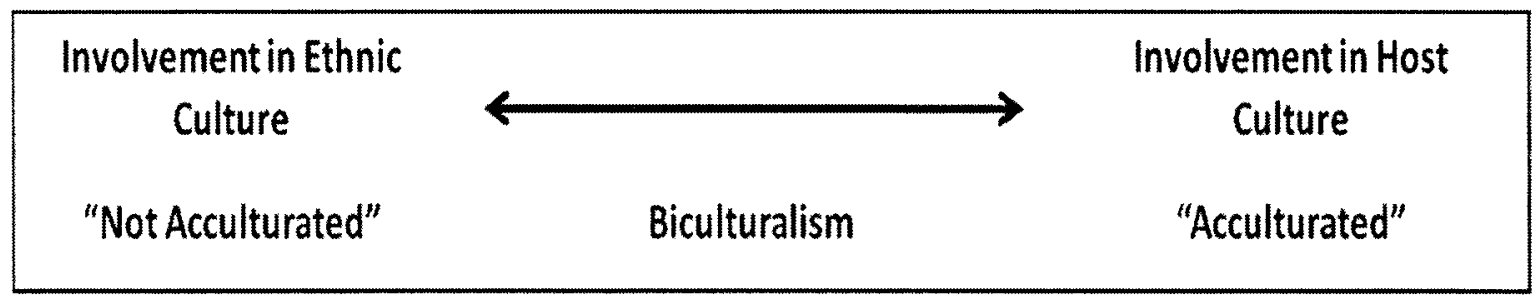

Figure 2. Bipolar Model of Acculturation (Nguyen \& von Eye, 2002) or Dual Cultural Unilinear Model (Kim \& Abreau, 2001)

Despite its contribution to the conceptualization and measurement of acculturation process, this model has one significant weakness. Some researchers (e.g., Cuellar, Arnold, \& Maldonado, 1995; Nguyen et al., 1999) argued that the unidimensional model 
(bipolar model) cannot distinguish individuals having high involvement with both cultures (i.e., biculturalism) from those having low involvement with both cultures. Even though this model has been criticized by many researchers, some researchers still conceptualize and measure individuals' acculturation process with a unidimensional model, since it is a useful means to explain immigrant experiences to date (Alba \& Nee, 1997).

In an effort to further understand biculturalism, Padilla (1980) proposed a new model of acculturation reflecting two major dimensions: (a) cultural awareness and (b) ethnic loyalty. According to this model, the acculturation process is based on (a) the knowledge and understanding of both the dominant culture and original culture, including the proficiency of the languages of each culture, knowledge of important historical events of each culture, and understanding of the artistic and musical forms of each culture; and (b) the ethnic loyalty which is composed of self-ascribed ethnicity, their friends' ethnic group membership, and their preferences for recreational activities (Padilla, 1980). Furthermore, this model suggested that individuals' lifestyles, social activities, clothing style, and food choices were decided by both the degree of cultural awareness and ethnic loyalty that they had for each culture.

Berry's bi-dimensional model. Unlike the unidimensional model, the bidimensional model commonly assumes that newcomers are capable of accepting two different sets of cultural values. This concept is consistent with cultural pluralism because in this model, individuals retain some characteristics of their original culture while at the same time they accept those of a new society. Consequently, this model takes into account orientation to both original and dominant cultures simultaneously (Berry 1990, 
1997, 2003). In addition, orientations toward one's own culture and toward a dominant culture should be independent of each other (i.e., orthogonal), providing a metric that allows identification of individuals as being oriented to one culture, two cultures, or neither culture.

Berry (1990), one of the most significant contributors to research on acculturation, first proposed a framework regarding acculturation strategies or patterns based on the bidimensional model. According to Berry $(1990,1997)$, given the ethnic diversity that exists in the United States today, assimilation into the dominant culture is not the only way of acculturation. There are other kinds of acculturation when considering two important issues. The first issue is whether individuals prefer to maintain or reject their own cultural values, identity, and heritage. The second issue is whether individuals accept or reject the values and identity of the dominant culture. As mentioned above, Berry took these two issues into consideration as independent attitudinal dimensions. Thus, this framework on the basis of bidimensional model allows individuals to report varying levels of acceptance and adherence to their original and to the dominant culture. Berry $(1990,1997)$ proposed four distinguishable strategies/patterns of acculturation with two questions: (a) "Is it considered to be of value to maintain cultural identity and characteristics? and (b) "Is it considered to be of value to maintain relationships with other groups?" (Berry, 1990, p. 245). The former question reflects that one's own ethnic identity/cultural identity and customs are of value and should be retained, while the latter question reflects the desirability of whether the relations with the host society are of value and should be sought. Based on the answers (yes or no) to these two questions, four types of acculturation strategies have been identified: (a) integration 
(yes/yes), (b) assimilation (no/yes), (c) separation (yes/no), and (d) marginalization (no/no). See Figure 3 below.

Maintaining Cultural Identity and Characteristics

\begin{tabular}{cc|c|c|}
\multicolumn{1}{c}{} & \multicolumn{1}{c}{ Yes } & No \\
\cline { 3 - 4 } $\begin{array}{c}\text { Maintaining } \\
\text { Relationship with } \\
\text { Other Groups }\end{array}$ & Yes & $\begin{array}{c}\text { Integration } \\
\text { (Yes//es) }\end{array}$ & $\begin{array}{c}\text { Assimilation } \\
\text { (No/Yes) }\end{array}$ \\
\cline { 3 - 4 } & No & $\begin{array}{c}\text { Separation } \\
\text { (Yes/No) }\end{array}$ & $\begin{array}{c}\text { Marginalization } \\
\text { (No/No) }\end{array}$ \\
\hline
\end{tabular}

Figure 3. Berry's Four Strategies of Acculturation (Berry, 1990, 1997)

The integration strategy/pattern, termed integration, occurs when individuals retain their own cultural values and identity while accepting those of the dominant culture, and, thus integration strategy represents biculturalism. Individuals who utilize the assimilation strategy do not want to maintain their own cultural values and identity while accepting those of a dominant culture.

The separation strategy occurs when individuals wish to accept their own cultural values and identity while avoiding the dominant cultural values. If such separation is enforced by the dominant group, it may be called "segregation." The last acculturation strategy is marginalization. Marginalization occurs when individuals neither maintain values and identity from their own culture nor accept values and identity from the dominant culture. 
However, the above four acculturation strategies (integration, assimilation, separation, and marginalization) only considered the perspectives of the non-dominant group without considering those of the dominant group. In other words, Berry's (1990, 1997) initial acculturation strategies were grounded in the assumption that newcomers from the non-dominant society can freely select a particular acculturation strategy without the consideration of the dominant group's attitude toward them. To fill this gap, Berry (2003) proposed four different acculturation strategies from the perspective of the dominant group in response to the four acculturation strategies of the non-dominant group's perspective. The additional four acculturation strategies include: (a) multiculturalism, (b) melting pot, (c) segregation, and (d) exclusion (or ethnocide).

First, when the dominant group is open to cultural diversity, an individual's integration strategy is termed "multiculturalism". Second, the assimilation strategy has been labeled differently depending on who chooses the strategy between the nondominant acculturating group (i.e., newcomers) and the dominant culture. When assimilation is forced by the dominant group, it was called a "pressure cooker"; but when it is chosen by the non-dominant group, it was called a "melting pot." Third, the "segregation" situation occurs when the dominant group forces the non-dominant group to use separation strategy. Last, when the marginalization is enforced by the dominant group, it is called "exclusion" (or ethnocide). Figure 4 displays eight acculturation strategies, considering both perspectives (dominant and non-dominant group). Two attitudinal dimensions in Figure 4, maintenance of heritage culture and identity and relationships sought among groups, intersect to classify acculturation strategies for both dominant and nondominant groups and are represented by bipolar arrows. 
Dimension 1:

Maintenance of Heritage Culture and Identity

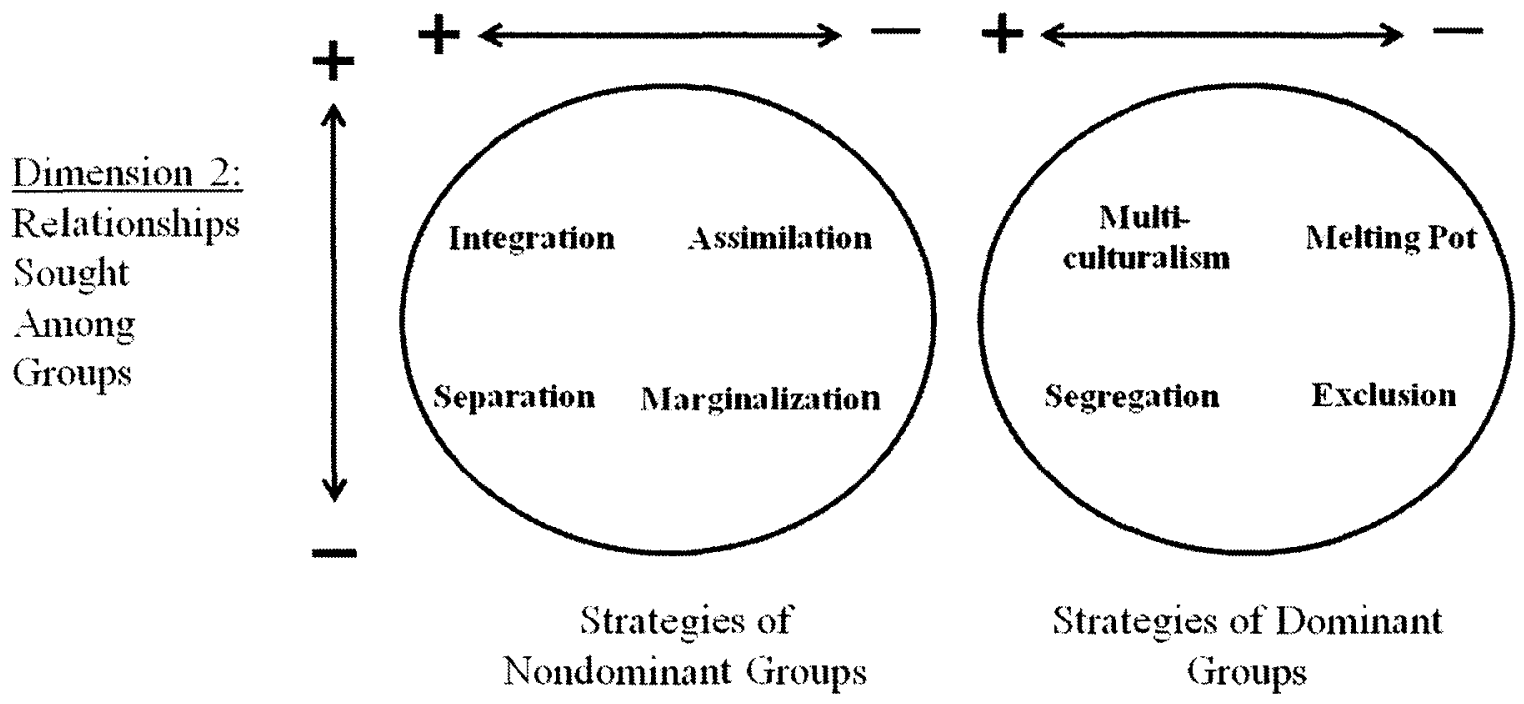

Figure 4. Acculturation Strategies Based on Two Dimensions - Perspectives of Nondominant Groups (left) and of Dominant Groups (right) (Berry, 2003, p. 23).

In addition to the aforementioned two models of acculturation, the acculturation model is recently being extended towards a multilinear-multidimensional measurement model that includes assessing acculturation in various spheres of society. The multilinearmultidimensional model of acculturation suggests that individuals can demonstrate not only involvement with their original cultures but also involvement with the dominant culture, and that the level of their involvement can vary independently (Berry 1990; Nguyen et al., 1999; Nguyen \& von Eye, 2002; Phinney 1990; Phinney et al., 2001). Furthermore, the multilinear-multidimensional model is an extension of the bidimensional model and incorporates acculturation measurements that represent changes in diverse situations reflecting varied cultures (Kim \& Abreu, 2001). This allows for multiculturalism which affirms that diverse cultures can exist together in a society 
(Phinney et al., 2001). "This complex model of acculturation potentially could lead to a fuller measurement model and better explain the complexities of the adaptation process experienced by ethnic minorities in the United States" (Kim \& Abreau, 2001, pp. 398399).

\section{Measurement of Acculturation}

Consistent with the evolution of the acculturation model from a unidimensional to a bi-dimensional model, acculturation measurement showed a similar evolution. In a study on reviewing acculturation instruments, Kim and his colleague (2001) reviewed published measurements regarding acculturation. They found a total of 33 instruments, including two for African American, three for Asian Americans, 23 for Hispanic Americans (e.g., Cuban Americans, Mexican Americans, and Puerto Rican Americans), three for Native Americans, one for Native Hawaiians, and one for all ethnic minority groups.

The researchers classified the above instruments based upon if they were a unilinear or bilinear model of measurement. The instruments based on the unilinear model were differentiated again whether they are a single culture or dual culture. Instruments with a single culture (i.e., mono-cultural unilinear) assess acculturation on a single continuum from one end (representing low attachment to one's culture) to the other end (representing high attachment to the dominant culture). Likewise, those instruments using a dual-cultural unilinear model assess acculturation on a single continuum, but one end of the continuum refers to high involvement with their culture of origin and low involvement with the host culture (i.e, low acculturation), while the other end is vice versa (i.e., high acculturation). Another different point from mono-cultural 
unilinear model is that the mid-point of continuum refers to biculturalism which represents high involvement in both the culture of origin and the host culture. A bilinear model of measurement produces two separate scores, "one representing level of adherence to the culture of origin and the other reflecting adherence to the host culture" (Kim et al., 2001, p. 399).

Kim et al. (2001) raised a critical point that most instruments reviewed were grounded in the unilinear model of measurement. Measurement of acculturation based on a unilinear model does not allow to separately or independently asses one's cultural orientation toward a host culture from his/her cultural orientation toward a culture of origin.

\section{Personal and Environmental Factors Affecting Acculturation}

A review of literature on acculturation indicated that one's acculturation process is dependent on a number of personal factors, such as: (a) age in migration, (b) gender (Beiser et al., 1988), (c) level of education (Jayauriya et al., 1992), (d) cultural distance (how dissimilar the two cultures are in language, attitudes, behaviors etc.; Ward \& Kennedy, 1992), (e) length of residence in a host country/generational status (Leao, Sundquist, Johansson, \& Sundquist, 2009; Tsai, Ying, \& Lee, 2000), and (f) motivations for moving (Richmond, 1993).

In a review of research on acculturation, Berry (1997) described the above individual factors affecting acculturation process. First of all, when people migrated to a host culture in early age, they are more likely to adapt to the host culture. For gender, females may have more difficulties in acculturation process than males. For educational level, the higher level of education an individual has, the fewer problems he/she has in 
the process of acculturation. In terms of cultural difference, the increase of a cultural difference between a host and a native country leads to poor adaption to the host country. For example, because immigrants from Asian countries have different cultural values from those of European and North American countries (e.g., collectivism in Asians and individualism in Americans) (see Triandis, Bontempo, Villareal, Asai, \& Lucca, 1988), Asian immigrants might not adapt well to American society.

Length of residence in the host country or generational status is a commonly used personal factor associated with the acculturation process of immigrants. The general findings from the literature indicated that the longer an individual resides in the host country, the higher the level of acculturation is (e.g., Ghuman, 1997; Oh, Koeske, \& Sales, 2002; Park, Paik, Skinner, Ok, \& Spindler, 2003).

Lastly, reasons for moving to a new country may also influence acculturation (Berry, 1997). There are two types of motivations: (a) pull motives and (b) push motives. These two motives are dependent on whether an individual voluntarily (pull motives) or involuntary (push motives) moves to the host country. The former case includes immigrants and international students, while the latter includes refuges. According to Kim (1988), individuals with voluntary motivation are more likely to adapt to the host country than those with involuntary motivation.

Aside from these individual factors, one's acculturation process can be also influenced by environmental factors such as: (a) national policies and (b) socializing agents. For national policies, individuals can adapt relatively easily adapted to a dominant society based on the following conditions: (a) the value of cultural diversity, (b) a lack of prejudice, and (c) positive reciprocal attitude (Berry \& Kalin, 1995). Socializing agents 
(e.g., family, peer groups, and media) also may play a critical role in affecting one's acculturation process. For example, an individual living in a family with strong value of original culture, having peers from one's original culture, and preferring to use mass media from original culture is likely to utilize separation strategy.

In summary, acculturation has evolved from the unidimensional model to the bidimensional model. Although the latter is currently predominantly utilized in these days to differentiate one's acculturation strategies (integration, assimilation, separation, marginalization), the former is still employed by some researchers to measure one's levels of acculturation instead of distinguishing the acculturation strategies. The literature has also shown that a variety of personal and environmental factors influence a newcomer's acculturation.

\section{Consumption Behavior among Ethnic Minority Groups}

Culture is recognized as the most important influence on consumption patterns/behaviors among ethnic minority groups (Cleveland \& Laroche, 2007). Due to people's varying values, lifestyles, and cultural backgrounds, it is evident that there are differences in consumption behaviors/patterns across cultures (Cleveland, Laroche, Pons, \& Kasto, 2009). Assael (1992) also posited that culture is the most powerful factor affecting consumer behavior, and cultural differences are reflected in products and services purchased. Similar consumption behaviors seem to emerge for consumers with a certain ethnic background, such as selecting similar products or services appropriate for their particular needs, while those from different ethnic backgrounds are likely to show certain different consumption behaviors from individuals with other ethnic groups. Thus, 
differential marketing efforts are required to meet their different needs and desires (Kim, Laroche, \& Joy, 1990).

Numerous studies have examined consumer behaviors with various ethnic minority groups (e.g., Hispanic American, African American, and Asian American) in crosscultural consumer and marketing related journals. A plethora of studies were based on two major constructs: ethnic identity and acculturation. Thus, a review of the literature in this area emphasizes studies examining ethnic minority groups' consumption behaviors with the two constructs of ethnic identity and acculturation. Before reviewing the literature, it is important to note that the concept of consumer acculturation in this review is a subcategory of the notion of acculturation in general. As such, it refers to the process displayed by consumers from non-dominant groups when they adapt to new values, attitudes, and behaviors in the consumption process in the dominant society (O'Guinn et al., 1986).

Penaloza (1994) originally proposed a conceptual model explaining Mexican immigrant consumer acculturation. According to this model, immigrants' individual differences, such as demographics (age, gender, social class, areas of residence, work status), length of residence in the U.S., ethnic identity, and environmental factors provide Mexican immigrants with differential skills to adapt to the consumption environment in the U.S. For example, older immigrants are more likely to maintain their prior culture and have more difficulties in adapting to the U.S. than younger immigrants. Further, there are two groups (Mexican and U.S. culture) of acculturation agents that consist of family, friends, retail business, media, schools, and churches. These agents from both Mexican and U.S. cultures serve to mediate the process of consumer acculturation. 
Based upon the previous two components (individual differences and acculturation agents), the consumer acculturation process is initiated and goes through three stages, including modeling, reinforcement, and social interaction. Lastly, this consumer acculturation process lead Mexican immigrants to three types of outcomes: (a) assimilate U.S. culture, (b) maintain their own culture (i.e., Mexican culture), and (c) express a combination of the two cultures.

In order to confirm his proposed model, the author utilized ethnographic research techniques using field notes based on observations in several sites and interviews with 23 Mexican immigrants from 14 households. Inconsistent with the proposed model, the findings revealed that the consumer acculturation process occurred through three different stages. Specifically, the consumer acculturation process began with individuals' movement from one nation to another (movement). They then experienced transition in terms of their consumption patterns from Mexican culture and U.S. culture (transition). In this process, the old consumption patterns they had acquired in Mexico played roles as bridges to those in U.S. culture. During the transition period, some Mexicans established new consumption patterns appropriate for the U.S., while others still maintained their own previous consumption culture (adaptation). Through these three stages (movement, transition, adaptation), Mexican immigrants showed four different types of consumer acculturation outcomes: (a) assimilation (assimilated many services and products related to American culture), (b) maintenance (maintained many aspects of consumption patterns associated with Mexican culture, (c) resistance (resisted many aspects of consumption patterns from both American and Mexican culture), and (d) segregation (Mexicans dwelled in places segregated from U.S. culture). The major finding of this study was that 
Mexican immigrants' consumption patterns in a new consumption environment were "much more complex than simply buying and using products associated with Americans or Mexican culture" (Penaloza, 1994, p. 49).

Jun, Ball, and Gentry (1993) proposed that both cultural identification (which is attitudinal in nature) and level of acculturation (which is behavioral in nature) were distinct constructs and played critical roles in explaining the different patterns of acculturation based upon Berry's (1990) acculturation model. With this concept, the authors suggested that the pattern of consumer acculturation of Korean sojourners (e.g., international students and their spouses) is different depending on whether they prefer to go back to Korea (preference for residency), whether they were raised in urban or rural areas, and whether they had much direct or indirect contact with the mainstream culture.

Data were collected from 26 males and 27 females. Cultural identification with the Korean culture was measured by four items, while acculturation level toward the American culture, consisting of two domains (language usage and media preference), was assessed by three items.

The findings indicated people who were willing to go back to Korea were more likely to identify with the Korean culture, and those who grew up in rural areas were more likely to resist adapting American culture. In addition, people having more direct contact with the dominant culture tended to adapt American culture more than their counterparts.

Lastly, cultural identification and acculturation levels were marginally associated with one another, indicating that the two constructs were distinct. Based on these findings, the authors suggested that various patterns of consumer acculturation 
(integration, separation, assimilation, marginalization, etc.) determined by cultural identification with the original culture and acculturation level toward the new culture should be used as a segmenting dimension for an ethnic group. In other words, the evaluation of characteristics and attributes of a product may vary depending on one's acculturation pattern.

Even though the results are meaningful to marketers, the study had two major limitations. First, there were issues with the validity of independent variables due to the use of single-item measures. Second, it may be difficult to generalize the findings due to the relative small sample size.

Unlike other studies, Gentry, Jun, and Tanshuaj (1995) first investigated whether acculturation models developed in North America can be generalized into the acculturation process occurring among the Muslim Thai and Chinese Thai. In this study, the authors attempted to compare Chinese and Muslim Thais with individuals of mainstream Thai culture. Data were collected from 90 Muslim Thai families, 93 Chinese Thai families, and 100 Thai families. Acculturation was measured by both the attitudinal dimension (i.e., cultural identity with home culture) and the behavioral dimension (i.e., ownership of consumer durables and language preference).

The findings revealed the attitudinal dimension (cultural identity) was relatively independent of the behavioral dimension (acculturation), similar to the results of studies from North America (e.g. Jun, Ball, \& Gentry, 1993). In terms of generational differences in cultural identity, the parent's cultural identification toward the original culture was higher than their child's cultural identification. This finding was also consistent with the 
result found in the United States, where parents were less likely to acculturate to the host culture.

Kang and Kim (1998) attempted to see if there were differences in the decisionmaking patterns for purchasing social clothes (clothes worn in social settings or for social event) among three major Asian American groups (Chinese, Japanese, and Korean). In addition, they investigated how Asian Americans' acculturation levels influenced decision-making patterns for purchasing social clothes. More specifically, in this study, ethnicity of three groups (Chinese, Japanese, and Korean) and acculturation with two groups (high acculturation group and low acculturation) served as independent variables, while reference group influence, media influence, and store attribute importance played roles in determining the decision-making patterns and served as dependent variables. Reference group influence included five different influence groups whose people rely on to buy social clothes: family/relatives, ethnic friends, American friends, ethnic coworkers, and American coworkers. Media influence included three major media sources which people rely on to buy social clothes, such as television, radios, and newspapers. Store attribute importance was operationalized as the level of importance people attached to each of three store attributes for buying social clothes, including upscale ambiance, convenience, and product-related appeal. A total of 481 useable surveys were collected from three groups (Chinese: 185, Japanese: 185, Korean: 144).

Kang and Kim (1998) found that Chinese respondents were more likely to rely on family/relatives than were their Japanese and Korean counterparts when they purchased social clothes. In terms of acculturation, people with high acculturation levels were more likely to rely on ethnic coworkers than those with low acculturation level. 
Speaking of media influence on purchasing social clothes, the low acculturation groups in Korean and Chinese were more influenced by both television and radio than were the high-acculturation groups. In contrast, for Japanese, the findings were reversed. As for store attribute importance, the results indicated that both Koreans and Chinese tend to consider product-related appeal more important than their Japanese counterparts. Additionally, people with a high acculturation level considered convenience more important than those with low acculturation level.

On the whole, the findings of the study revealed the clear message that marketers or advertisers targeting Asian Americans should not consider all Asian groups as homogeneous. Specifically, the findings revealed that each of three Asian groups (Chinese, Korean, and Japanese) displayed significant differences in terms of how much they were affected by the different sources of reference group, media, and store attribute importance. In addition to the differences based upon ethnicity, differences presented based on the level of acculturation. However, this study had a significant limitation because acculturation was measured using the unidimensional perspective (from low acculturation to high acculturation) even if the current trend of conceptualizing and measuring acculturation is consistent with the bi-dimensional perspective.

In their study on consumer acculturation, Maldonado and Tansuhaj (2002) sought to segment a local Mexican market into the three acculturation categories Berry (1990) suggested (Assimilation, Separation, Integration), and then attempted to investigate how Mexican Americans showed different responses in consideration of their brand choice between American and Mexican brands according to Berry's acculturation categories. Of the 410 survey questionnaires mailed to Mexican Americans, 116 responded and 
completed the survey. According to Maldonado and Tansuhaj (2002), acculturation categories consisted of two dimensions, attitudinal and behavioral. The attitudinal dimension represents one's ethnic identity, while the behavioral dimension refers to involvement/participation in ethnic and host cultural behaviors. With this perspective, they measured Mexicans' acculturation using the Cultural Life Style Inventory (CLSI; Mendoza, 1989) consisting of the two dimensions. To measure brand choice, participants were asked to select between Mexican brands and the U.S. brands from six products. Their level of brand choice was determined by the percentage of times a participant chose Mexican brands over the U.S. brands.

Fifty-eight, 31 and 20 participants were classified into the assimilation, integration, and segregation categories, respectively. The results of the ANOVA showed that individuals in the assimilation category tended to choose the U.S. brands more than Mexican brands. Those in the integration category showed similar proportions in selecting between Mexican (52\%) and the U.S. brands (48\%). Finally, those in the segregation category were more likely to choose Mexican brands than those in the assimilation category, but there were no significant differences between individuals in the segregation and those in the integration categories. Maldonado and Tansuhaj (2002) concluded from their analysis that Berry's acculturation taxonomy was a useful tool to understand consumer acculturation and its influence on brand choice while segmenting the market into acculturation categories.

Based upon a socialization model developed by Moschis and Churchill (1978), Xu, Shim, Lotz, and Almeida (2004) attempted to (a) determine how perceived parental acculturation level and cultural identification and ethnic-friendship orientation influenced 
ethnic identity among Asian American young adults, to (b) examine the effect of ethnic identity on culture-specific consumption behavior (e.g., food and entertainment behavior), and (c) to see if situational factors (e.g., the existence of ethnic friends) strengthened the relationship between an individual's ethnic identity and his/her culturespecific consumption behavior.

Using the combination of mail surveys with Web-based surveys, a total of 336 college students from 20 different states (mails survey: 128, Web-based survey: 208) participated in the study. To assess participants' ethnic identity, parental acculturation and cultural identification, ethnic-friendship orientation, and culture-specific consumption behavior, four different types of instruments were employed. For data analysis, structural equation modeling and MANOVA were performed to test a series of hypotheses.

Looking at the results of the study, perceived parental cultural identification and ethnic-friendship orientation significantly influenced ethnic identity among Asian American young adults. This ethnic identity in turn affected their culture-specific consumption behaviors, such as food and entertainment purchases. Asian American young adults who had a stronger sense of ethnic identity were more likely to consume ethnic food and attend ethnic entertainment activities. Furthermore, this study found that perceived parental acculturation level and ethnic-friendship orientation directly influenced culture-specific consumption behavior.

Another important finding of this study was that a situation factor (i.e., the existence of ethnic friends) influenced an individual's culture-specific consumption behavior, regardless of the degree of ethnic identity. This result indicated that Asian 
American young adults were more likely to partake in culture-specific consumption behaviors when they were with friends of the same ethnicity than when they were with Caucasian friends. Specifically, Asian American young adults who had the same ethnic friends tended to consume ethnic food, listen to ethnic music, and to attend ethnic movies.

Cleveland, Laroche, Pons, and Kasto (2009) investigated patterns of cultural adaption of Lebanese-Canadians residing in a host culture (i.e., French-Canadians) as manifested in consumption of original ethnic and host/dominant culture foods as well as the relationship between ethnic identity and acculturation. More importantly, this study also examined how ethnic identity and acculturation influenced both traditional ethnic food consumption and dominant culture food consumption. Of 250 participants, 166 questionnaires were usable for data analysis. Lebanese ethnic identity and acculturation were measured with seven dimensions (e.g., language use, media exposure, social interaction, identification and pride, customs and values, family structure and sex roles, and culture maintenance), respectively. Participants also reported their consumption frequencies for Lebanese and French-Canadian food products.

The relationship between ethnic identity and acculturation was found to be low and negatively correlated. In terms of food consumption, traditional food consumption was positively correlated with Lebanese ethnic identity, but not negatively correlated with acculturation, indicating that overall, traditional ethnic food consumption may be retained despite acculturation to the dominant culture. Dominant culture food consumption was positively correlated with ethnic identity as well as acculturation, indicating that overall 
consumption of dominant culture food increased, even with higher levels of ethnic identity.

Through structural equation modeling, the authors found that ethnic identity and acculturation were distinct constructs (i.e., bi-dimensional model of acculturation) that cannot be positioned as two extremities of the same continuum (i.e., bipolar model of acculturation). Thus, this study provided strong support for bi-dimensional model of acculturation.

Lastly, based on the relative influence of ethnic identity and acculturation on various food consumption frequencies, seven different patterns of cultural adaption (i.e., acculturation) were found: (a) non-cultural, (b) pure acculturation, (c) acculturation dominant, (d) balanced integration, (e) identification dominant, (f) pure identification, and (g) assimilation. This typology for acculturation patterns refined previous categorizations suggested by Mendoza (1989) and Berry (1990). Cleveland concluded that based on Berry's acculturation patterns, non-cultural and pure acculturation patterns were close to marginalization; acculturation dominant, balanced integration, and identification dominant patterns were similar to Integration; pure identification was closed to Separation; and assimilation was the same as Berry's acculturation pattern.

\section{Summary}

As several studies outlined above, literature on consumption behaviors among ethnic minority groups was strongly grounded in two main constructs: ethnic identity and acculturation. The major results found in the literature were that these two constructs played critical roles in categorizing their consumption behaviors/patterns into four groups in accordance with Berry's (1990) acculturation typology: Integration, Assimilation, 
Separation, and Marginalization. In addition, researchers found that ethnic identity and acculturation were distinct constructs (i.e., bi-dimensional model) that cannot be positioned as two extremities of the same continuum (i.e., unidimensional model). Therefore, the literature provided more strong support for bi-dimensional model of acculturation than unidimensional model of acculturation.

\section{Sport Consumption Behavior among Ethnic Minority Groups}

Traditionally, professional sport franchises and leagues in North America have not dedicated much attention to minorities while focusing on mainstream market (i.e., White Americans). Recently, sport organizations have begun to recognize the large opportunities that ethnic minority groups provide for extending fan bases and increasing sales revenues (Clarke \& Mannion, 2006). In the similar manner, sport marketing and management research has also begun to focus on ethnic minorities as vital and potential market segments. Thus, the following review of literature explains sport consumption behaviors among ethnic minority groups.

Armstrong (1998) suggested 10 strategies for marketing sport to Black consumers. First, in order to effectively market to Black consumers, the marketer should understand not only the salience of Black cultural nuances but also the uniqueness of Black consumers. Thus, sport organizations need to hire professionals who have expertise and familiarity with the Black consumer market. Second, given the media habits and preferences of Black consumers, it is a viable strategy for sport marketers to use Black media outlets in order to increase the number of Black sport fans. Third, sport marketers should use advertisements and promotional messages containing and reflecting themes and content appealing to Black consumers. Fourth, sport marketers need to allow Black 
consumers to identify with their sport organization through Black employees such as athletes and coaches. This strategy may facilitate positive perceptions of and attitudes toward sport organizations and then lead to more favorable responses to the products and services the organizations provide.

Fifth, partnering and affiliating with Black vendors and businesses as corporate sponsors may increase attendance among Black consumers. Sixth, including the products and services at sport events that are appropriate for Black consumers is an important factor in attracting them into the sport venue. Seventh, to increase attendance among Black consumers, sport managers should engage in socially responsible/cause-related marketing because Black consumers are considered socially conscious individuals. For example, a professional sport franchise can donate a certain proportion of its revenues from sport events to a charitable social organization associated with the Black community.

Eighth, it is important for sport managers to identify appropriate product distribution channels to reach Black consumers. Ninth, considering that individuals' thoughts and behaviors develop during the early stage of life, sport managers should involve Black youth in their organization through grassroots programs. Utilizing this strategy, children may perceive the organization positively, providing an opportunity for their involvement to translate into loyal fans/consumers when they become adults. Lastly, in order to form a support network with the Black community, identifying influential Blacks is a critical factor. Armstrong (1998) concluded in her argument that though Black consumers have common characteristics, there are diverse differences among them 
depending on demographics (e.g., gender, income, age, education) and psychographics (e.g., ethnic identity, acculturation level, attitudes).

McCarthy (1998) provided several comments and suggestions about how sport managers might most effectively approach the Hispanic population, the fastest growing population in the United States. First, the continuous increase in the number of young Hispanic adults might be an important potential target market for both spectator and participant sports. Second, because most of the Hispanic population is living in the cities and large metropolitan areas, the Hispanic population in those urban areas is a major target market for professional sport franchises, particularly Major League Baseball and Major League Soccer, where many Hispanic players compete.

Third, in order for sport marketers to more effectively approach the Hispanic population, they should focus on advertising in the Spanish language rather than the English language. In addition to these, McCarthy (1998) proposed that community-based marketing programs/strategies involving Hispanic players are effective vehicles for reaching out to the Hispanic market.

With the recognition that international college students in general may be an important segmented target market to increase revenues for intercollegiate athletic departments, Kwon and Trail (2001) attempted to distinguish their sport consumption behaviors from those of American college students. More specifically, the purposes of their study were to determine if international college students differed from college students from the U.S. in (a) frequency of attendance and television spectatorship for college football and basketball games, (b) psychological motives for watching the two sports, and (c) levels of identification with the college football and basketball team. 
Data were collected from 130 American students and 104 international students in a Midwestern university in the United States. To measure their motivations for sport spectating, the Sport Fan Motivation Scale (Wann, 1995) was adopted and modified. They used five fan motives from the original eight motives: eustress, group affiliation, betting on sport (economics), self-esteem, and aesthetics. For identification with the teams, six items were adopted from a survey created by Trail and James (2001). The frequency of attending and watching the institution's football and basketball games were measured by four items (two items for each).

The results from MANOVAs and ANOVAs indicated the only significantly different motive between international students and American students was aesthetics ( $p$ <.05). Eustress was the primary motive for sport spectating for both international and American students. In terms of the frequency of sport spectating behaviors (i.e., attending games and watching them on TV), American students attended football and basketball games more frequently than international students, while there were no statistically significant differences in the frequency of watching the games on TV between the two. These results indicated that international students' interest level for football and basketball games is evident "but is not being expressed by attendance at the sporting events" (Kwon \& Trail, 2001, p. 153). For the level of identifications with the teams, while no significant differences between American and international students existed, the international students' identification with the football and basketball teams was considerably high and almost close to the identification level of American students.

The findings from this study implied that international students are an underdeveloped market that has the potential of increasing revenues for collegiate 
football and basketball teams. However, this study was limited to only assessing international students' consumption behaviors for football and basketball. Although college football and basketball are viewed as the popular sports for American students, international students may have the highest interest in different kinds of sports. Additionally, cultural variables, such as acculturation and ethnic identity, may be critical tools to more thoroughly explain the unique consumption characteristics of international students for college sports.

In a study on the impact of race on sport consumption, Armstrong and Peretto Stratta (2004), examined whether there were significant differences in several aspects of sport consumption between Black and White consumers of professional women's basketball teams from two different areas in the U.S. Those aspects included (a) communication tools the two races widely used for team or game information, (b) their purchase patterns regarding games, (c) their game attendance frequency, and (d) motivation factors influencing game attendance.

Data collection occurred in two different regions of the U.S.: one in the Midwestern market and the other one in the southern market. For the Midwestern market, a total of 710 usable questionnaires (606 Whites and 104 Blacks) were collected, while a total of 1,361 usable questionnaires (716 Blacks and 568 Whites) were collected from the southern market. The communication tools used for team/game information were more similar than dissimilar for the Black and White spectators in the Midwestern and southern markets. Specifically, most of both groups in the Midwestern market primarily relied on team schedules to obtain information about game and team. In the southern market, the most frequently used communication tool for both White and Black spectators was word- 
of-mouth advertising. One of the key findings regarding communication tools was that Black spectators in the southern market relied more on radio to obtain information about the team's games.

In terms of ticket purchase patterns, a notable finding was that White spectators were much more likely to engage in advanced ticket purchase than Black spectators in both markets. In analyzing game attendance frequency between Blacks and Whites, conflicting results emerged from each market. For the Midwestern market, there were no significant differences in the basketball game attendance frequency between Black and White Spectators. On the other hand, there were significant differences in the southern market, indicating that Black spectators attended at a higher frequency than White spectators.

Armstrong and Peretto Stratta (2004) related these findings to Gouke's (1987) argument, suggesting that Blacks and Whites tend to buy a product designed for the general market at similar rates, whereas Blacks mostly purchase a product mainly designed for the Black consumer market. In this study, the Midwestern team may be perceived as using a product designed for the general market because the racial composition of the team was almost balanced between White and Black (i.e., five Black athletes and four White athletes). The finding from the Midwestern market supported this argument because there were no differences in game attendance frequency between Black and White. In contrast, the team in the southern market may be perceived as a product specifically designed for the Black consumers in that the team for the southern market was predominantly composed of Black athletes (i.e., eight Black athletes and four White 
athletes). Thus, the Black consumers were more likely to attend the games. The result from the southern market supported this argument.

In terms of motivations for game attendance decisions, there were significant differences in two motivations -- the opportunity to support the league and the opportunity to see the opposing team -- for game attendance decision between Black and White spectators in the Midwestern market. Specifically, Black spectators considered the two factors more important to the attendance decisions than White spectators. For the southern market, there were significant differences in seven motivations -- entertainment, social atmosphere, adult giveaway, children giveaway, special promotions, quality of food, and ticket price - for game attendance decision between Black and White spectators, indicating that Black spectators considered the seven factors more important to the attendance decisions than White spectators.

Although this study provided insight into the similarities and differences of sport consumption behaviors between Blacks and Whites from two different geographical regions, other important potential factors (e.g., demographic, social, and psychological factors) influencing their sport consumption behaviors were not included. With this limitation, Armstrong and Peretto Stratta (2004) insisted that future research should examine these factors along with race.

Claussen, Ko, and Rinehart (2008) sought to emphasize and demonstrate the significance of connecting demographic information with culturally relevant information when sport managers market and promote to culturally diverse groups of sports participant consumers. In their explanation, Chinese-Americans were inclined to buy their tickets ahead of a sport event, while Hispanic groups preferred to purchase tickets 
right before the sport event (Brenner, 2004). In addition, Hispanic sport fans living in predominantly Spanish-speaking households were more likely to be World Cup soccer fans and less likely to be NFL fans than those who live in English-dominated households (King, 2007). Considering these differences in sport consumption behaviors among culturally and ethnically diverse groups, Claussen et al. (2008) insisted that a cultural diversity approach to sport consumer behavior is meaningful for better understanding culturally and ethnically diversity. Failure to do so from the perspective of sport organizations can be costly.

The authors used the diversity of action sports (e.g., the X Games, the Gravity Games, roller hockey, skateboarding, BMX bike) participants as an example to explain how connecting "a cultural diversity perspective with the use of demographic information might be especially effective in that sport industry niche" (Claussen et al., p. 68). Based upon the cultural diversity approach in sport organizations (Doherty \& Chelladurai, 1999), Claussen et al. (2008) concluded that research on sport consumer behavior, particular in participant sports, should also adopt this approach in order to more effectively promote sport participation by culturally and ethically diverse consumer groups.

Despite increasing interest in sport consumers with different cultural and ethnic backgrounds, Ko, Claussen, Rinehart, and Hur (2008) suggested recent studies on ethnic minority groups in sport marketing and management have primarily centered on African Americans (Armstrong, 1998, 2002a, 2002b; Armstrong \& Peretto Stratta, 2004) and Hispanics (Harrole \& Trail, 2007; McCarthy, 1998), rather than Asian Americans. In order to better understand Asian American sport consumers, Ko et al. (2008) attempted to 
investigate their demographic profiles and unique cultural characteristics. Furthermore, they depicted the misconceptions about marketing toward Asian Americans and suggested effective and appropriate marketing strategies targeting Asian American sport consumers.

In terms of demographic profiles, Asian Americans' demographic characteristics can be summarized in several ways: (a) diverse subgroups within Asian Americans (e.g., Chinese, Japanese, Korean, Indians, Vietnamese), (b) geographic locations (most live in big cities and three major states), (c) better educated than average people from the United States ( $44 \%$ of Asian Americans have undergraduate degrees or higher), (d) diverse language use (two-thirds of Asian Americans are immigrants whose native language is not English), and (e) strong family structure due to a collectivist cultural background.

According to Ko et al. (2008), the strong family orientation derived from the Confucian collectivist cultural tradition affects Asian American consumer behavior in several ways. First, when they purchase products, they usually consider family and societal expectations rather than focusing on their own individual needs and desires. Second, when they purchase products and search for information about products, Asian Americans are more affected by parents and peers than other ethnic groups, such as Hispanics and African Americans (Singh, Kwon, \& Pereira, 2003). In addition to these common characteristics of Asian Americans, the authors illustrated misconceptions about marketing to Asian Americans. One of key misconceptions was that people tend to refer Asian Americans as a homogeneous group without differentiating people by ethnic and cultural backgrounds and characteristics among subgroups. 
In terms of implications for sport marketers, the authors suggested that sport organizations should more actively engage in recruiting high profile Asian players in order to draw more Asian American sport fans. This may be a key strategy for increasing fan-player identification. Another implication was that sport organizations should sponsor Asian American organizations or participate in culturally relevant community events based on relationship marketing. As additional implications, Ko et al. (2008) suggested that considering Asian Americans are not generally impulse buyers and are very sensitive to brand image and quality, sport marketers need to develop promotional strategies associated with these characteristics.

While research on ethnic minority groups in sport management has focused almost exclusively on sport consumers with culturally and ethnically diverse backgrounds, Choi (2010) first examined athletes' ethnicity in relation to sport sponsorship. LPGA tournaments have been primarily dominated by Asian players during the last fifteen years. Supposedly, both the LPGA and its sponsors were growing unhappy with the explosion of Asian winners and LPGA golfers lacking English skills. Thus, Choi's study attempted to investigate how a major LPGA sponsor (i.e., Anheuser-Busch) responded to and capitalized on this new situation (the rapid influx of Asian players to the LPGA) in meeting its corporate sponsorship objectives and strategies.

Based on a case study design, 11 semi-structured interviews were performed with several persons associated with Anheuser-Busch (A-B) Company. At the same time, field notes from two observations at A-B events and documents related to A-B were also used to analyze data. This study revealed three major themes: (a) selling more beer is A-B's clear objective, (b) increased marketing opportunities can be derived from ethnic 
diversity on the LPGA, and (c) the existence of Asian players brings the LPGA up to a much more competitive level. Overall, the existence of numerous Asian players in the LPGA clearly impacted A-B's sponsorship objectives and strategies. Unlike the expectation that language and cultural barriers Asian players face may negatively impact the LPGA tour and its sponsors, Anheuser-Busch actively utilized the LPGA's growing ethnic diversity for its own competitive advantage. These findings imply that it is necessary for the other LPGA sponsors to customize their sponsorship objectives, strategies, and tactics appropriately to ethnic groups.

\section{Summary}

In this section, sport consumption behaviors of various ethnic minority individuals in the U.S. were reviewed. The articles outlined above demonstrated that different marketing and promotional strategies are needed to meet varying needs and desires of diverse ethnic minority groups, such as Black (Armstrong, 1998; Armstrong \& Peretto Stratta, 2004), Hispanic (McCarthy, 1998), and Asian consumers (Ko et al., 2008). Most previous studies, however, have solely focused on Blacks and Hispanics rather than Asians (Ko et al.). In addition, while prior studies merely provided comments and recommendations for effective marketing strategies targeting specific ethnic minority groups, very limited scientific and empirical evidence is available on how ethnic minority individuals are really showing sport consumption behavior. Aside from sport consumers with culturally and ethically diverse backgrounds, Choi (2010) found that ethnic minority athletes can positively influence the professional league or team for which the athletes currently play and its sponsors. Thus, it is important for both the league/team and its sponsors to utilize ethnic minority players to develop a niche market. 


\section{Ethnic Identity and Acculturation in a Sport Context}

According to Harney (1985) (as cited in Pons, Laorche, Nyeck, \& Perreault, 2001), the selection of a particular sporting event represents a powerful cultural meaning for an individual in that it allows him/her to identify with a specific culture. From this point of view, "a specific sport can be identified as being ethnically specific" (Pons et al., 2001, p. 235), such as ice hockey to the Canadians, soccer to the Brazilians, Taekwondo to the Koreans, football to people from America and so on. Therefore, the role of sport in a multicultural and multiethnic environment is important in helping individuals to identify themselves with a particular culture.

In spite of the arguments that consumption behavior is considered a cultural phenomenon and cultural variables (i.e., ethnic identity and acculturation) can be associated with consumption behavior (McCracken, 1986; Pons et al., 2001), relatively little attention was paid to studying the important roles cultural and ethnic issues play in sport participation and consumption. In particular, very few empirical studies suggested evidence on how ethnic identity and acculturation influence sport identification and consumption behavior.

Pons et al. (2001) sought to investigate how ethnic identity and acculturation impact sporting events consumption and orientation among Italian Canadians living in Canada. Furthermore, they attempted to examine whether "immigrants can adapt to a new cultural environment by acquiring traits of the host culture as well as by maintaining traits of their own culture" (Pons et al., 2001, p. 232) through sport event consumption. In addition to these, assuming that a specific sport event has been identified as being specifically ethnic-based, they hypothesized that Italian Canadians would be more likely 
to be soccer oriented, while French Canadians would be more likely to be hockey oriented.

Data were collected from 210 participants consisting of 107 French Canadians and 103 Italian Canadians. The results from ANOVA revealed that French Canadians were more hockey oriented and consumed more hockey events than Italian Canadians. For soccer, Italian Canadians were more oriented toward soccer and consumed more soccer events than their French Canadian counterparts. In analyzing the impact ethnic identity and acculturation have on sporting event consumption and orientation, the Italian Canadian sample alone was used for the analysis. The results from stepwise multiple regressions showed that the consumption of and orientation toward soccer (i.e., Italian attribute) were influenced by ethnic identity, while the consumption of and orientation toward hockey (Canadian attribute) were influenced by acculturation.

Furthermore, Pons et al. (2001) found no significant difference in soccer orientation between the high-acculturated Italian Canadians and those of the low-acculturated. In the case of hockey, the orientation toward hockey of high-acculturated Italian Canadians did not differ significantly from the French Canadians. These findings supported the bidimensional acculturation model, indicating that an individual can obtain traits of the dominant culture, while at the same time maintain traits of his/her culture of origin. Italian Canadians simultaneously maintained their ethnic identity with a sport having Italian attributes (i.e., soccer) and acquired traits of the host culture through a sport representing Canadian attributes (i.e., hockey).

In a qualitative study, Stodolska and Alexandris (2004) explored the role of recreational sport in the adaption processes of recent immigrants from Korea and Poland 
living in Chicago and Urbana-Champaign using Portes and Zhou's (1993) segmented assimilation framework. According to the segmented assimilation framework, immigrants may follow different routes in the process of adaption to their host society. First, immigrants adopt mainstream values and expectations and become integrated into the American middle class. Second, some immigrants may assimilate the values and attain substandard economic levels of the American underclass. This route is often taken by racially distinct immigrants. Third, many immigrants choose to adapt and promote their economic success by consciously preserving their ethnic values and promoting their ethnic group solidarity.

Through semi-structured in-depth interviews from 30 first generation immigrants (19 Koreans and 11 Polish), the results indicated that middle class Korean and Polish immigrants were more likely to use recreational sport participation as a way to acculturate to the life of the White American middle class mainstream than their bluecollar counterparts. However, the findings did not confirm the second assimilation method that some immigrants assimilate values of the American underclass. For their assimilation path, some immigrants revealed that participation in recreational sports helped solidify their ties with their ethnic communities or served as a factor facilitating retention of ethnic identity. Furthermore, they found that sport participation fostered intergroup contacts and broke down obstacles between first generation immigrants, host society White Americans, and other ethnic group members. Stodolska and Alexandris (2004) suggested that there have been limited numbers of studies on in recreational sport participations among ethnic minorities, and future research needs to focus on such issues 
as recreational sport preferences, needs, motivation, and barriers that ethnic minority groups have.

Similarly, Lee (2005) explored the cultural meanings immigrants placed on sport participation. She conducted a study using in-depth interviews at a large Midwestern university. Data were collected from six Korean American women: four from second generation (those born in the U.S.) and two from 1.5 generation (those born in Korea but immigrated to the U.S. when they were young). The interview data indicated that "their feelings of uncertainty and ambiguity regarding their status in the United States” (p. 485) were focal to the experiences of the Korean American women. This unsettled position in American society influenced the Korean American women in selecting the types of sports in which they participated. Some interviewees selected sport participation as a critical vehicle in the process of finding their position in mainstream society with three different strategies: (a) assimilation and acculturation into the mainstream American society, (b) maintenance of the ethnic culture (i.e., Korean), and (c) rejecting both the American and Korean culture.

One interviewee who was a lacrosse player selected lacrosse as a way of rejecting both American and Korean cultural stereotypes. For example, the lacrosse player disliked American football because it is for the mainstream American society. Two other interviewees, however, used sport as a tool for connecting and developing a sense of membership with their culture of origin. For instance, they sought to affiliate with a Korean traditional martial art (i.e., Tae Kwon Do) as means for strengthening "ties to their ethnic background and often expressed their sense of ethnic pride" (p. 488). Lastly, another interviewee used a dance sport and football as a means to assimilate into the 
mainstream American society. These sports gave her opportunities to be with White Americans.

Harrolle and Trail (2007) attempted to investigate how acculturation (represented by identification with dominant culture: the US ID) and ethnic identity (identity with their own culture: Latino ID) influenced identification with sports in general and identification with particular sports, including American football, baseball, basketball, hockey, and soccer, among Latinos living in the U.S. This study tested four hypothesized models (Model A, B, C, and D) across identification with sports in general and identification with the five particular sports.

In Model A, acculturation and ethnic identity combine to affect sport identification. Model B illustrated that acculturation alone influences sport identification without the direct effect of ethnic identity on sports identification, but acculturation and ethnic identity are still correlated. In contrast to Model B, Model C depicted that ethnic identity fully influences sport identification without the direct effect of acculturation. Model D suggested that ethnic identity and acculturation both independently influence sport identification without the correlation between the two.

A sample of 294 participated in the study from the southeastern US. To measure acculturation, six items were derived from the Abbreviated Multidimensional Acculturation Scale (AMAS). For measuring ethnic identity, six items were derived from a revised Multigroup Ethnic Identity Measure (MIEM). Identification with sports in general and the five particular sports was assessed by the Points of Attachment Index (PAI). Data were analyzed using structural equation modeling. The above four structural 
models (Model A, B, C, and D) were tested individually for goodness of fit across identification with sports in general and the five particular sports.

In the analysis of identification with sports in general and identification with American football and baseball, Model B was chosen based upon several goodness of fit indices (RMSEA and $x^{2} / d f$ ) and the number of residuals greater than .10 . More specifically, acculturation explained approximately $4.8 \%, 12.0 \%$, and $7.4 \%$ of the variance in identification with sports in general, American football and baseball, respectively. In the analysis of identification with basketball, Model A was chosen as the most appropriate model. For identification with hockey, Model $\mathrm{C}$ was chosen as the most appropriate model and showed that ethnic identity explained only $2.0 \%$ of the variance in identification with hockey. Lastly, the result from soccer identification showed that although Models A, B, and C revealed good fits, none of the models were chosen because both paths from acculturation and ethnic identity to soccer identification were not significant. Harrolle and Trail (2001) concluded in this study that for Latinos, acculturation and ethnic identity had a greater impact on identification with American football than identifications with the other sports examined.

From a theoretical perspective, Harrolle and Trail (2007) concluded that ethnic identity and acculturation only explained a limited amount of the variance in sport identification with Latinos. From a marketing perspective, although acculturation only explained a small amount of the variance in identification with American football $(12.0 \%)$, baseball $(7.4 \%)$, and basketball $(2.0 \%)$, the findings indicated that sport marketers should create marketing campaigns targeting Latinos with high levels of acculturation in order to foster their consumption behaviors in those sports. 
However, this study failed to examine identification with ethnic-specific athletes and teams. For example, it is plausible that Latinos living in the U.S. are likely to identify with Latino athletes. Furthermore, they are more likely to identify with sport teams having Latino players. In addition, because this study examined a whole Latino community, we cannot know what the different relationships in the four models exist depending on a specific ethnic subgroup (i.e., Cubans, Mexicans, Puerto Ricans).

While the studies above attempted to investigate the relationships between sport participation or spectatorship and acculturation which are related to sport services, Shin (2010) first attempted to investigate the effect of acculturation on a sporting good (golf clubs). Specifically, the author sought to examine the effect of Korean American golfers' acculturation levels (less acculturated vs. moderately acculturated) on seven consumerdecision making styles (CDMS) in relation to purchasing golf clubs. The seven consumer-decision making styles include: (a) quality consciousness, (b) brand consciousness, (c) confusion by overchoice, (d) novelty consciousness, (e) brand loyalty, (f) price consciousness, and (g) style/technology consciousness. The results of this study indicated that there were only significant differences in two decision-making styles (novelty consciousness and brand loyalty) across acculturation levels. This study, however, measured acculturation on the basis of a unidimensional model and thus did not consider two dimensions of acculturation (original culture and host culture) simultaneously.

\section{Summary}

In spite of the importance of ethnic identity and acculturation to newcomers in adapting a host society, very few studies have examined the relationships between these 
two cultural factors and their sport-related behaviors and attitudes in sport management and marketing realms. Some previous studies demonstrated how acculturation and ethnic identity influence sport-related behaviors and attitudes of ethnic minority individuals (Lee, 2005; Pons et al., 2001). In addition, sport can play an important role in adapting to a dominant society (Stodolska \& Alexandris, 2004). Further, sport consumption behaviors (Pons et al.) and identification (Harrole \& Trail, 2007) of ethnic minority individuals were found to be influenced by levels of ethnic identity and acculturation. However, it is important to note that the relationship between the two cultural variables and fan identification were different depending on the type of sports (Harrole \& Trail).

\section{Identification in a Sport Setting}

To better understand the construct of fan identification in a sport context, it is essential to reexamine social identity theory since sport fan identification is mainly based upon social identity theory as in ethnic identity. Social identity refers to "that part of an individual's self-concept which derives from his [her] knowledge of his [her] membership in a social group (groups) together with value and emotional significance attached to that membership" (Tajfel, 1978, p. 63). As such, this theory posits that an individual's self-concept consists of two distinct aspects: one is a personal identity and the other is a social identity (Tajfel \& Turner, 1986). Personal identities reflect an individual's attributes, skills, or abilities (e.g., competence, intelligence, talent), while social identities reflect group categorizations/memberships/affiliations to which individuals belong (Tajfel, 1982; Tajfel \& Turner, 1986). Through such group affiliations, individuals seek to maintain positive self-esteem or self-image (Tajfel, 1981). Typically, when individuals are asked who they are, they tend to describe themselves in association 
with some social groups to which they belong. Applying this into a sport context, it is not unusual for a sport fan to describe him/herself as a New York Yankees fan, a baseball fan, or Alex Rodriguez fan by presenting his/her affiliation with certain groups or objects.

In a sport context, Trail, Anderson, and Fink (2000) defined "identification as an orientation of the self in regard to other objects including a person or group that results in feelings or sentiments of close attachment" (p.165-166). While initial research on sports fan identification (or attachment) has solely focused on identification with a specific team (i.e., team identification) (e.g., Branscomb \& Wann, 1991; Fisher \& Wakefield, 1998; Sutton, McDonald, Miline, \& Cimperman; Wann \& Branscomb, 1990, 1993), Trail, Robinson, Dick, and Gillentine (2003) and Robinson and Trail (2005) extended the concept of team identification into other sport-related objects. They suggested that individuals might also identify with or attach to other sport-related objects, such as the players, the coach, the community, the sport in general, the university, and the level of sports (e.g., college basketball vs. professional basketball). Thus, sport fan identification includes not only identification with a team, but also identification with various sportrelated objects.

\section{Points of Attachment}

Many researchers have noted the need for research on points of attachment and examined them from different perspectives. Hunt, Bristol, and Bashaw (1999) described points of attachment as targets of schema based on information processing theory. According to them, the targets of the schema refers to various sport consumptive objects, such as sport itself, a team, a league, a player or a coach. For example, one may attend or watch LA Lakers games on TV because his/her target schema may be at a team level (LA 
Lakers), a sport level (basketball), a player (e.g., Kobe Bryant), and/or a coach level (e.g., Phil Jackson). Furthermore, Hunt et al (1999) proposed that individuals' targets of schema (i.e., points of attachment) toward sport-related objects can be illustrated by a hallow process. If one is a fan of a specific sport, for example, he/she then become a fan of a team on the sport and then a fan of a player. Indeed, Fisher and Wakefield (1998) demonstrated this hallow effect, indicating that individuals' identification with a athletes or a sport can bring about their identification with the team and ultimately leading to sport consumptive behaviors, such as attendance and purchase of licensed team products.

Consistent with Hunt et al. (1999), Mastsuoka and Fujimoto (2002) suggested that there might be varying targets of psychological commitment for sport fans. Specifically, it is possible for fans to attach to different sport consumptive points, including coaches, players, peer fans, the team, or the community of the team (Mastsuoka \& Fujimoto). In their study, they found that there existed attachments to the community, the team, and a specific player, and these points of attachment were related to game attendance or sport media consumption.

In a study on the development of sport interest inventory at the 1999 Women's World Cup, Funk, Mahony, Nakazawa, and Hirakawa (2001) found that some people attended the soccer events due to interest in soccer (i.e., attachment to sport), while other attended the events due to interest in specific players (i.e., attachment to players). In addition, Wann, Tucker, and Schrader (1996) found there are some factors affecting the inauguration, maintenance, and termination of identification with sport teams. Of these factors, attachments to the players and the geographical reason (attachment to the 
community) are two of the most important factors for the continuation of identification with sport teams.

Robinson and Trail (2005) sought to examine if there were significant differences in points of attachments based upon gender and types of sport events (intercollegiate football, women's basketball, and men's basketball). They found that female spectators attached to players and sports greater than male spectators. For types of sports, significant differences in four points of attachments (players, sports, coaches, and university) were found by types of sports events. They also showed that regardless of gender and types of sports, sport fans were most highly attached to the following two points: team $(M=4.95)$ and sport $(M=4.90)$ on the seven-point Likert scale.

Even though it is obvious that sport fans and spectators identified with not only a team, but also other sport-related objects, including a player, a sport, a coach, a university, a community, and level of sport, there have been no research on points of attachment among ethnic minority groups living in the U.S., with the exception of Harrole and Trail's work (2007). However, Harrole and Trail have solely focused on identification with sports rather than identification with players. According to Min and Kim (2009), Asian immigrants tend to maintain their ethnic identity by rooting for teams or players from their native countries. For example, Japanese Americans are likely to attend or watch Seattle Mariners' games because of Ichiro Suzuki. Likewise, Korean Americans tend to attend or watch Cleveland Indians' games because of Shin Soo Choo. These demonstrate that immigrants, particularly in Asian population, are inclined to identify with ethnic-specific players (i.e., attachment to players) and eventually attend or watch a team's game with athletes from their culture of origin. 
According to Harney (1985) (as cited in Pons, Laorche, Nyeck, \& Perreault, 2001), the selection of a particular sporting event represents a powerful cultural meaning for an individual in that it allows him/her to identify with a specific culture. From this point of view, "a specific sport can be identified as being ethnically specific" (Pons et al., 2001, p. 235), such as ice hockey to the Canadians, soccer to the Brazilians, Taekwondo to the Koreans, football to people from America, and so on. This demonstrates that attachment to a specific sport might vary depending on ethnic groups. Applying this concept to attachment to popular sports in both native country and host country, ethnic minority groups living in the U.S. may be more likely to identify with the popular sports in the native country than those of the host country and eventually read more articles about and watch the popular sports in the native country through the Internet and TV respectively. For example, an international college student (e.g., Korean) studying in the U.S. might be more identified with Korean Baseball League (KBL) and follow news about the sport than popular sports in the host country (the U.S.), such as NBA, MLB, and NFL. It may also be true that his or her levels of identification with and consumption behaviors for sports in both the native and host country may depend on their length of residence or generational status.

The two points of attachments above (a sport and player) are particularly meaningful when considering the concepts of acculturation and ethnic identity. Within the sports marketing and management literature, however, there are no comprehensive empirical studies designed to examine the relationships among acculturation, ethnic identity, the above two points of attachment (player and sport), and sport consumption behavior among Asian populations living in the U.S. 


\section{Fan Identification and Its Outcomes}

A literature review on sport fan identification showed that team identification is a considerably powerful phenomenon in spectator sports. Specifically, team identification influenced a variety of behavioral, affective, and cognitive outcomes and led to sports fans' consumption behavior (Dietz-Uhler \& Lanter, 2008).

In terms of affective outcomes, many researchers examined the effects of team identification with affective consequences, such as sport fans' level of arousal (i.e., anxiety) and emotional responses (e.g., happiness, enjoyment, life satisfaction, sadness, depression). For example, Wann and his colleagues (2002) found that team identification was positively correlated with positive affects, such as happiness, pleasantness, and satisfaction, after their team win. In addition, highly identified fans reported greater enjoyment than those in low identification while watching their team win (Wann \& Schrader, 1997).

The cognitive consequences of sport fan identification included team knowledge, perceptions of teams, and attributional bias (Dietz-Uhler \& Lanter, 2008). Wann and Branscomb (1995) found that highly identified fans of a men's basketball team are more knowledgeable of the team and the sport than those in low identification. Furthermore, Wann and Dolan (1994) investigated attributional bias among highly identified fans after the team win or lose and found that highly identified fans revealed more internal attributions (e.g., home-team players) about the team's performance after a win, but more external attributions (e.g., opposing team players, referees) about the team's performance after a loss. 
Dietz-Uhler and Lanter (2008) noted that behavioral aspects are the most widely investigated and easily observable consequences of sport fan identification, including self-esteem responses, various sport consumption behaviors, and aggression. One behavioral consequence associated with sport fan identification is self-esteem responses, such as BIRGing and CORFing. BIRGing (Basking in reflected glory) is a way individuals increase their self-esteem through connecting with successful others (i.e., winning teams), whereas CORFing (Cutting-off reflected failure) deals with individuals' tendency to maintain their self-esteem by distancing themselves from unsuccessful others (i.e., losing teams) (Ciadini et al., 1976; Snyder, Lassegard, \& Ford, 1986) (as cited in Wann \& Branscombe, 1990). For example, "when a favorite team wins, people often say that "we won", while suggesting that "they lost when the favorite team does not perform well" (Mahony, Nakazawa, Funk, James, \& Gladden, 2002, p. 5).

Furthermore, fan identification is considered as an important concept in predicting sports fans' various consumption behaviors. Wann and Branscomb (1993) found that individuals in high identification with a team attended more home games and reported a greater likelihood of attending away games than less identified individuals. In addition, highly identified fans reported they would pay more money to purchase regular season, playoff, and championship game tickets. A study carried out by Fisher and Wakefield (1998) supported the results. They found that team identification was significantly related to frequency of game attendance and the purchase of licensed merchandises.

Based upon a thorough review of literature on sport spectators and fans, Trail, Anderson, and Fink (2000) developed a theoretical model that examined influences on future consumption behavior of sport spectators. This model consisted of six main 
factors: (a) nine motivations for spectator and fan consumption behavior (achievement, knowledge acquisition, social interactions, family, drama/eustress, aesthetics, escape, physical attractiveness of the participant, physical skill of the participant), (b) level of team identification, (c) expectancies for event experiences (i.e., beliefs about the quality of play in an event) or outcome (i.e., win or lose), (d) confirmation or disconfirmation about event experiences and outcome, (e) self-esteem maintenance, such as BIRGing and CORFing, and (f) affective states or reactions (e.g., happiness, pleasantness, satisfaction, alienation, sadness). In the model, it appears that level of identifications plays a crucial role in future spectator consumption behavior.

In order to confirm the theoretical model that Trail et al. (2000) proposed, Trail, Fink, and Anderson (2003) empirically tested that model. However, the model did not explain the variance of spectators' future consumption behavior well.

\section{Athletic Achievement and Sport Consumption Behaviors}

In a sport context, it is obvious that athletic achievement of either a team or a player is an important factor affecting various sport consumption behaviors, including game attendance, merchandise purchase, watching games on TV and reading sportrelated articles via Internet. Indeed, the literature demonstrated the relationship between athletic achievement and sport consumption behaviors. Zhang, Pease, Hui, and Michaud (1995) assessed factors affecting attendance of NBA games and found that both home team and opposing team performances (e.g., win/loss records, league standing) were significant predictors of game attendance in the current season. In recent year, Davis (2009) analyzed attendance records of 12 National League teams during 27 years (19792005 ) in order to investigate the relationship between team success (winning) and game 
attendances of MLB. The results revealed that winning is a significant determinant of game attendances.

While Zhang, Pease, Smith, Lee, Lam, and Jambor (1997) argued that since most previous studies on marketing of sports events have solely centered on major league sports, they attempted to identify factors affecting attendance of a minor league sport (in this study, hockey). The findings revealed that while home team performance was a significant predictor of game attendance, the opposing team performance was not a significant predictor. Using economic demand models, DeSchriver and Jensen (2002) sought to empirically identify determinants affecting spectator attendance at NCAA Division II football games. A key finding of the study was that the effect of winning on game attendance changed during the season. In other words, the influence of winning percentage in the current season increased as the season progresses, whereas the effect of winning percentage in the past season decreased as the season progressed.

Considering that there has been little research on external factors influencing game attendance at women's sporting events, Shackelford and Greenwell (2005) found that previous winning percentage was the most significant predictor for game attendance at NCAA Division I women's sporting events.

Lastly, in a study on the relationship between team performance, performance of star players and Major League Baseball (MLB) attendance, Rivers and DeSchriver (2002) found that the presence of star players had no significant relationship to MLB attendance. However, the on-field success of a team (i.e., the numbers of years since the team's last playoff appearance) was significantly related to attendance. From these findings, Rivers and DeSchriver (2002) suggested that if a star player does not contribute to the team 
performance, the star player rarely affect on attendance. Furthermore, Gladden, Miline, \& Sutton (1998) suggested that a team success (winning) and athletic achievement of star player are critical components of brand equity by increasing merchandise sales, ticket sales, enhancing the mood at games, and exposing TV broadcasts.

\section{Summary of Literature Review}

This study is designed to delve into the development of Sport Consumption Model for Asian populations living in the U.S. using the constructs of ethnic identity and acculturation. Because Asians in the U.S. are regarded as one of the fastest growing population segments, as well as a population having a disproportionately large economic power in terms of buying power and household income, a review of literature began with various attributes of Asians in the US such as high income and high level of education. Among over 30 different countries, Asians from four countries - China, Japan, Korea, and Taiwan - are familiar with sports as an option of entertainment due to their relatively well-developed sport industry and economic power.

In order to develop the Sport Consumption Model for U.S. Asians, various studies and theories on ethnic identity and acculturation were reviewed. The review showed that the two terms, ethnic identity and acculturation, are frequently used interchangeably (Nguyen et al., 1999). However, Phinney (2003) clarified that acculturation is viewed as a broader construct, including an array of attitudes, behaviors, and values that change with contact between two or more different cultures. Ethnic identity is viewed as the most important part of the acculturation process that focuses on the subject sense of belonging to an ethnic group. 
A review of literature on acculturation theories indicated that the acculturation process can occurr either unidimensionally or bidimensionally. According to the unidimensional perspective, as one moves to a new society, he/she loses values and identity of his/her original over time. In other words, adaptation to the dominant culture is seen as necessarily accompanied by a weakening of ties to one's culture of origin so that an acculturated individual almost gives up his or her culture of origin (Nguyen, Messe, \& Stollak, 1999). The bidimensional perspective, on the other hand, suggests that when individuals move to a new society, they maintain some characteristics of their original culture while at the same time accepting those of a new society (Berry, 1990). Based upon the bidimensional acculturation perspective, Berry proposed four different types of acculturation strategies: integration (high identity in both a native culture and a host culture), assimilation (high identity in a host culture, but low in a native culture), separation (high identity in a native culture, but low in a host culture), and marginalization (low in both cultures).

The next area of review in the literature examined general consumption behavior (e.g., clothing, food) among ethnic minority groups based on ethnic identity and acculturation. In spite of some arguments, common findings of the previous studies indicated differences in consumption behaviors across culture due to varying values, lifestyle, cultural backgrounds, and acculturation strategies or levels. That is, a certain ethnic group has culture-specific consumption behaviors/patterns. Likewise, in the context of sport consumption, Pons et al. (2000) found that a certain ethnic group is identified with and consume for a cultural-specific sport (e.g., hockey to the Canadians, soccer to the Italians). Overall, the literature on both general consumption and sport 
consumption behaviors among ethnic minority groups indicated that the two constructs of ethnic identity and acculturation played important roles in understanding their consumption behavior.

The final area of review in the literature examined sport fan identification which is a major predictor of sport consumption behavior. The literature review has shown that the research trend of sport fan identification is moving from team identification to identification with other sport-related objects (i.e., identification with the player, the coach, the community, the sport). The literature review has shown that fan identification was a critical predictor for various sport consumption behaviors, such as game attendance, purchase of licensed merchandises, and media consumption. 


\title{
CHAPTER III
}

\begin{abstract}
METHOD
The purpose of this chapter was to discuss the methodology used to test the Research Questions posed in Chapter 1. This section explains: (a) the research design, (b) the selection of participants, (c) the data collection procedures, (d) the instruments used to collect data, and (e) the data analysis strategies.
\end{abstract}

\section{Purpose of the Study}

There were two purposes for the current study. The first purpose was to develop and test Sport Consumption Models for the Asian population living in the U.S. based on ethnic identity and acculturation. For this purpose, this study explored the relationships among ethnic identity, acculturation, three points of attachment [identification with an ethnic player (IEP), identification with a popular sport in a native country (IPSNC), and identification with a popular sport in a host country (IPSHC)], and three sport consumption behaviors [consumption for a team with an ethnic player (CTEP), consumption for a popular sport in a native country (CPSNC), and consumption for a popular sport in a host country (CPSHC). The three proposed models for this purpose were presented in Figures 1-1 (Ethnic Player Model), 1-2 (Native Sport Model), and 1-3 (Host Sport Model).

The second purpose of this study was to examine the impact of the four different acculturation strategies (integration, assimilation, separation, marginalization) on the 
three different points of attachment and the three consumption behaviors for the respective points of attachment. For this purpose, the study included the three different identifications: (a) identification with ethnic player(s) (IEP), (b) identification with a popular sport in a native country (IPSNC), and (c) identification with a popular sport in the U.S. (the host country in this study) (IPSHC); and the three different sport consumption behaviors: (a) consumption for a team with an ethnic player (CTEP), (b) consumption for a popular sport in a native country (CPSNC), and (c) consumption for a popular sport in the host country (CPSHC).

\section{Research Questions}

To accomplish the two purposes mentioned above, this study contained four primary Research Questions. Research Questions 1, 2, and 3 were developed for the first purpose of the study (developing and testing Sport Consumption Models for the Asian population), while Research Question 4 was developed for the second purpose of the study (examining the effects of acculturation strategies on sport fan identifications and consumption behaviors). The first three Research Questions for the first purpose are as follows:

1. What is the relationship among ethnic identity, acculturation, identification with an ethnic player, and consumption behavior for a team with the ethnic player?

2. What is the relationship among ethnic identity, acculturation, identification with a popular sport in a native country, and consumption behavior for the sport in a native country? 
3. What is the relationship among ethnic identity, acculturation, identification with a popular sport in the U.S., and consumption behavior for the sport in a native country?

The fourth Research Question, which aligns with the second purpose, and its three sub-questions are listed below:

4. What are the relationships of four acculturation strategies (integration, assimilation, separation, marginalization) with each of the three different targets of identification and consumption behavior for the respective identification, while controlling for the effect of length of residence in the U.S.?

4-1: Are there significant differences in identification with an ethnic player and consumption behavior for a team with the ethnic player among four acculturation strategies regardless of length of residence in the U.S.?

4-2: Are there significant differences in identification with a popular sport in a native country and consumption behavior for the sport among four acculturation strategies regardless of length of residence in the U.S.?

4-3: Are there significant differences in identification with a popular sport in the U.S. and consumption behavior for the sport among four acculturation strategies regardless of length of residence in the U.S.?

\section{Research Design}

This study incorporated a cross-sectional survey design with no manipulation of research variables. A cross-sectional survey design has several advantages including the following: (a) it is helpful in explaining the characteristics of a large population; (b) 
standardized questionnaires ask participants exactly the same questions and infer the same intent to all participants giving a certain response, thus strengthening the quality of the results; (c) it is useful in collecting data from large samples; and (d) can be easily changed (Babbie, 2007).

\section{Participants}

The target population for this study was Asian people 18 years of age or older living in four large cities in the U.S.: Los Angeles, Houston, Seattle, and Cleveland. These four cities were chosen since Asian athletes from the four countries (China [Houston - Yao Ming, Houston Rockets], Japan [Seattle - Ichiro Suzuki, Seattle Mariners], Korea [Shin Soo Choo - Cleveland Indians], and Taiwan [Los Angeles Hong Chiu Kuo, Los Angeles Dodgers]) are currently playing or played in those four cities either with MLB or NBA teams. Targeting the Asians whose culture of origin are the four countries above would be also more meaningful in that it would provide great opportunities for sport practitioners to increase revenue sales and expand their fan bases. Furthermore, Asians from the four countries are more likely to consume sports because the four countries are relatively well developed in the sport industry compared to other Asian countries. Thus, the current study focused on these four ethnic subgroups. However, as mentioned in Chapter I, the four subgroups (Chinese, Japanese, Korean, Taiwanese) were not directly compared to each other in this study. Rather, data were analyzed in aggregate.

More specifically, the target population included Asian people who identify their culture of origin as one of the four Asian countries located in the Northeast Asian region: (a) China, (b) Japan, (c) Korea, or (d) Taiwan. Data from Chinese, Japanese, Korean, and 
Taiwanese samples were collected from Houston, Seattle, Cleveland, and Los Angeles, respectively. Furthermore, potential participants were either Asian Americans (citizen or alien resident) or Asian sojourners (i.e., Asian international students, business people temporarily working in the US, etc.). In other words, any people of Asian descent legally living in the four cities (not tourists) were qualified to participate in the current study.

The preferred method of sampling is to choose a sample representation of the population based on a probability sampling technique including simple random sampling, cluster sampling, stratified sampling, or systematic sampling (Ary, Jacobs, \& Razavieh, 2002). Yet, if there is a situation where enumeration of the population is impossible, Ary et al. (2002) suggests that non-probability sampling such as purposive sampling, accidental sampling, and so on, can be utilized.

Of the non-probability sampling techniques, this study utilized the judgmental sampling method to gather potential participants. This sampling technique is a method in which researchers select a sample to be observed based on the researchers' judgment and knowledge about the target population, its elements, and the purpose of the study. This type of sampling is regarded as a valid alternative to a probability sampling when it is unrealistic to obtain a truly random sample, which frequently happens in the field of social science (Babbie, 2007).

In social science research, face-to-face, telephone, and online surveys are the most widely used data collection methods for survey research design. This study utilized both online and off-line methods in order to administer the survey instruments. Even though each type of data collection method has its pros and cons, the combined method of data collection design involving online and face-to-face is useful in reducing effects or bias of 
data collection methods on the survey results while at the same time balancing cost (Groves, Fowler, Couper, Lepkowski, Singer, \& Tourangeau, 2004).

After discussing data collection methods with sport administration faculty members, a decision was made to work through officers of Asian college student associations and Asian community/cultural centers based on the culture of origin in each of the four cities. For the Japanese sample, for example, officers of Japanese student association(s) and community center(s) in Seattle were contacted. Similar procedures were conducted for other three ethnic groups at each of the three remaining cities (Chinese from Houston, Korean from Cleveland, and Taiwanese from Los Angeles). As results of contacts for data collection permission, five of the seven Asian student associations in the four cities allowed for the data collection (two for Taiwanese and one for each of the remaining subgroups), but for Asian community/cultural centers, Taiwanese and Chinese community/cultural centers in L.A. and Houston respectively did not coorporate with the data collection. Therefore, data were collected through 7 organizations (five student associations and two community/cultural centers) in the four cities.

Asian student associations and community centers were chosen for two reasons. First, student associations or ethnic community centers are commonly selected for data collection places in the studies on ethnic minority groups (e.g., Harrole \& Trail, 2007, 2010; Jang, Kim, Chiriboga, \& King-Kallimanis, 2007; Jun, Ball, \& Gentry, 1993). Second, since ethnic minority populations are often difficult to identify by any single approach and a single-source sampling frame may lead to bias (Curry \& Jackson, 2003), the researcher recruited participants from both Asian student associations and Asian 
community centers in the four cities. Also, collecting data from multiple locations increases generalizability of the finding. Thus, officers of the two Asian community centers and five Asian student associations in the four cities were contacted by emails for data collection.

Although the justifications above for data collection are reasonable, it is plausible that collecting data from Asian student associations and community centers may influence the levels of participants' ethnic identity and acculturation since being members of these associations and centers may mean potential subjects are already highly involved with and participating in their own ethnic organizations. Thus, the scores on ethnic identity are likely to be high, whereas the scores on acculturation are likely to be low compared to when data collection occurs in general public place. Due to this possibility, the researcher checked means of skewness values on the two variables (ethnic identity, acculturation).

\section{Sample Size}

With regard to sample size in structural equation modeling (SEM), the sample size should be determined based on how the complexity of the model is (Kline, 2005). In other words, the greater the number of free parameters, the larger sample size is needed. Kline suggested that sample size in SEM should be greater than 20 times the number of free parameters (Kline). Given that each of the three models related to the first purpose of the current study has a total of 10 freely estimated parameters (i.e., 4 factor variances, 5 path coefficients, and 1 covariance, respectively), a minimum of 200 participants from the four groups was required. 
For the sample size determination for MANCOVA, a G Power priori Power Analysis was employed (Faul, Erdfelder, Lang, \& Buchner, 2007). To estimate adequate sample size, three different elements are required: $f^{2}$ (effect size), number of groups, and dependent variables. A medium effect size, by the G Power program's convention

provided by Cohen (1988), $f^{2}=.15$, was employed. Each of three MANCOVAs in the current study has four groups and two dependent variables. To achieve a power of .95 for a MANCOVA with four groups and two dependent variables in a medium effect size, a total sample of 80 was required.

In addition to the above two sample size calculations, the researcher made an effort to increase the sample size in order to generalize the findings of the current study to the target population. The total number of members in the seven organizations was approximately 948. Based upon Salant and Dillman's (1994) suggestion, the adequate sample size for a population of 1,000 using the $95 \%$ confidence level with less than sampling error $\pm 5 \%$ was about 278 .

\section{Data Collection Procedures}

Data collection for this study combined two major survey methods: off-line survey (i.e., paper survey) and online survey. For the paper survey, the researcher contacted officers working in Asian community centers or Asian student associations in the four cities, and asked them to administer the survey questionnaire. After approval was given to administer the survey, each officer was given further background about the study such as the purpose of the study. In addition, each officer was instructed to distribute the survey questionnaire, along with an email from the researcher explaining the study (see Appendix A). Then, the survey questionnaire, consisting of a preamble and instruments 
used in this study, was provided to participants. The preamble (see Appendix B) including consent form provided a brief explanation of the purpose of the study and importance of the survey and emphasize that all responses were treated in a confidential manner. It takes approximately 10 minutes for a respondent to compete the survey in either English or their own language.

According to Dillman (2007), online survey websites like Formsite or Survey Monkey allow researchers to easily access a sample and analyze data. For the online surveys, the researcher contacted officers working in Asian college student associations or community centers in the four cities as with off-line survey and asked them to send their members email notification about the online survey. More specifically, online survey participants were recruited by sending an email including an invitation to participate in the study and a link to an Internet website on which the survey questionnaire was posted. The first page of the link provides the same preamble included on the face-to-face survey. After reading the preamble, respondents were asked to indicate whether they are from one of the following four countries: China, Japan, Korea, and Taiwan. Only participants indicating their culture of origins as one of the four countries continued to fill out the survey.

A modified version of the methods suggested by Dillman (2007) was utilized to administer the online survey. First of all, participants were sent a pre-notification email two days before distribution of the actual survey. According to Dillman, use of a prenotification letter is a useful way of alerting the subject to the upcoming survey.

A reminder email was sent to individuals who have not completed the survey one week after the initial emailed survey. A final reminder email was sent to individuals who 
had not completed the survey one week after the reminder emails. Similar procedures on the initial emails were followed with the reminder emails and the final reminder emails. All of participants received a thank you note after completing the survey. The online surveys were administered via a third-party company (www. surveymonkey.com). The data were stored on a server provided by the online company and the data were downloaded at the end of data collection.

Although the combined method of data collection design involving online and faceto-face survey distributions has some advantages, some researchers have raised questions whether different results exist between the two different formats of data collection and found no statistically significant differences between the two methods (Cronk \& West, 2002; Hancock \& Flowers, 2001). As such, the researcher conducted a series of one-way ANOVAs to determine if there are significant differences in the main variables for the current study (i.e., ethnic identity, acculturation, identifications with sports and players, sport consumption behaviors for the respective identification) between the two data collection methods. In addition, considering that data were collected from two different sources (Asian student association and Asian cultural center) in each of the four cities, it is plausible that participants fill in the same survey twice, but different places if they are members of both organizations (e.g., Japanese student association and Japanese community center in Seattle). To avoid this possibility, the researcher asked officers in data collection organizations to mention, during the data collection, if they already filled out the survey before at somewhere either using hard copy or electronic copy.

\section{Instrumentation}


The survey questionnaire consisted of five instruments to measure the followings: ethnic identity, acculturation, points of attachment, sport consumption behavior, and demographics. The instrumentation process included the following steps: (a) item selection and modification, (b) item review by a panel of experts, (c) back translation, and (d) pretesting.

\section{Item Selection and Modification}

Based on the literature, items for measuring ethnic identity, acculturation, points of attachment (identifications with players and sports), and sport consumption behaviors were selected and modified. The items believed to be the most appropriate for this study and showing sound psychometric properties were initially selected and then modified to suit the current study context.

Ethnic identity. To measure ethnic identity, the Multigroup Ethnic Identity Measure - Revised (MEIM-R) (Phinney \& Ong, 2007) was employed. The MEIM was originally developed by Phinney (1990) to measure three aspects of ethnic identity: sense of belongings and positive ethnic attitudes ( 5 items), ethnic identity achievement ( 7 items), and ethnic behaviors or practices ( 2 items). The original 14-item MEIM instrument, however, was modified by Robert et al. (1999). Using exploratory and confirmatory factor analyses, they removed two items and found two factors represented by 12 items: an exploration factor ( 5 items) and a commitment factor ( 7 items). The exploration factor is associated with efforts to learn more about one's ethnic group and involvement in his/her ethnic cultural practices. The commitment factor included items dealing with a positive affirmation of one's ethnic group as well as a sense of belonging and commitment. The 12-item MEIM instrument was revised again by several pilot 
studies conducted by Phinney and Ong (2007). Using interviews, focus groups, and exploratory factor analysis, they deleted two items from the exploration factor, resulting in retaining three items, and four items from the commitment factor, resulting in retaining three items. Thus, the MEIM-R consists of the two factors with six items: exploration (3 items) and commitment (3 items).

Considering the definition of ethnic identity in the current study, using the 3-items for the commitment factor is appropriate for the current study. More specifically, all three items for the commitment factor are associated with a sense of belongingness and attachment to an ethnic group which cover the definition of ethnic identity in the current study. Internal consistency of the 3-item commitment factor has been established by acceptable coefficient alpha values in previous studies (Harrole \& Trail, 2007; $\alpha=.89$; Phinney \& Ong, 2007; $\alpha=.78$ ). Construct validity (i.e., convergent validity) for the commitment factor was also established by the Average Variance Extracted (AVE) of .63 in a prior study (Harrole \& Trail, 2007). According to Hair, Anderson, Tatham, and Black (1998) and Fornell and Larcker (1981), the AVE, which indicates the overall amount of variance in the indicators accounted for by the latent construct, needs to exceed the variance explained by measurement error. An AVE value above .50 indicates the scales have good convergent validity (Fornell \& Larcker, 1981; Hair, Anderson, Tatham, \& Black, 1998).

The response format can affect variability of a measurement scale (DeVellis, 2003). For example, if a measurement scale has too many response options, participants might not be able to distinguish the difference between the response options. In contrast, if there are too few options, the measurement scale cannot differentiate among participants with 
different underlying judgments. Typically, since a seven-point scale is the most appropriate response format for variability (Krosnick \& Fabrigar, 1997), the researcher modified the number of response options from a 5-point Likert-type scale to a 7-point Likert-type scale, ranging from strongly disagree (1) to strongly agree (7).

Acculturation. The literature on acculturation showed that many acculturation scales are designed for specific ethnic group: African-Americans (Landrine \& Klonoff, 1994; Snowden \& Hines, 1999), Hispanics (Cuellar, Arnold, \& Maldonado, 1995; Mendoza, 1989), Asians (Barry, 2001; Chung, Kim, \& Abreu, 2004; Suinn, RickardFigueroa, Lew, \& Vigil, 1987), Native Americans (Rezentes, 1993), and multiple ethnic groups (Stephenson, 2000). The two most widely used measures of acculturation for Asian populations are the Suinn-Lew Asian Self-Identity Acculturation (SL-ASIA; Suinn et al., 1987) and the Asian American Multidimensional Acculturation Scale (AAMAS; Chung et al., 2004). Even though the two scales consist of relatively similar items, the AAMAS was chosen for this study due to its bidimensionality or multidimensionality rather than unidimensionality.

The original AAMAS asked respondents to rate each of the 15 items on three different cultural dimensions or referent groups (i.e., culture of origin, other Asian Americans, European Americans) and thus creating 45 items. Accordingly, the original AAMAS consists of three subscales: (a) AAMAS - Culture of Origin (AAMAS - CO), (b) AAMAS - Asian Americans (AAMAS - AA), and (c) AAMAS - European Americans (AAMAS - EA). For the purpose of the current study, however, the two subscales with 30 items were chosen, the AAMAS - CO and the AAMAS - EA. The reason that the AAMAS-AA was excluded was that the subscale's referent group is other 
Asian Americans. In other words, since acculturation strategies in the current study should be determined not by the comparison between culture of origin (e.g., China, Japan, Korea, Taiwan) and other Asian Americans' culture, but by the comparison between culture of origin and American culture, the AAMAS-AA was excluded.

Within the two cultural dimension scales, each scale measures four domains of acculturation: Cultural Identity (six items), Language (four items), Cultural Knowledge (three items), and Food Consumption (two items). A sample item for cultural identity is "How much do you feel you have in common with people from (culture of origin or American culture)?", that for language is "How well do you speak the language of (culture of origin or America)?", that for cultural knowledge is "How knowledgeable are you about the culture and traditions of (culture of origin or American)?", and that for food consumption is "How often do you actually eat the food of (culture of origin or American)?" These four domains are the most widely investigated domains with regard to measuring acculturation. In addition, measuring the four domains allowed for assessing both behavioral and psychological aspects of acculturation. For the response format, the researcher also modified the scale from a 6-point Likert-type scale to a 7 point Likert-type scale to increase variability of the scale, ranging from Not at All (1) to Very Much (7). Two questions were worded in the opposite direction. The coefficient alphas of the AAMAS - CO were Cultural Identity $(\alpha=.79)$, Language $(\alpha=.84)$, Cultural Knowledge $(\alpha=.77)$, and Food Consumption $(\alpha=.71)$, while the coefficient alphas of the AAMAS - EA were Cultural Identity ( $\alpha=.78)$, Language $(\alpha=.82)$, Cultural Knowledge ( $\alpha=.71)$, and Food Consumption $(\alpha=.71)$ (Chung et al., 2004). 
Given the two purposes of the current study, the AAMAS was utilized differently. To measure the level of acculturation (i.e., a unidimensional acculturation approach) for the first purpose of the study, six items (items 16-21) were adopted pertaining to the American identity from the AAMAS-EA. To separate the four different acculturation strategies (i.e., integration, assimilation, separation, marginalization) for the second purpose (i.e., a bi-dimensional acculturation approach), all of the 30 items from the AAMAS-CO and AAMAS-EA were adopted. The four acculturation strategies were determined by the criteria listed in Table 1.

In order to classify the four acculturation strategies, some scholars have championed the use of midpoint split method rather than the median split since the former approach allows for a more direct approximation of participants' preferred acculturation strategies (Dona \& Berry, 1994). However, the midpoint split would increase the likelihood of generating an unbalanced sample size among four acculturation groups/strategies (Castro, 2003). To make comparisons with balanced sample size, which is the focal point of the second purpose for the study, the median split method was appropriate to classify acculturation strategies.

Table 1.

Four Acculturation Strategies Based on The AAMAS-CO and The AAMAS-EA

1) Integration $=$ Score above the AAMAS-CO median and score above the AAMAS-EA median.

2) Assimilation = Score below the AAMAS-CO median and score above the AAMASEA median. 
3) Separation $=$ Score above the AAMAS-CO median and score below the AAMAS-EA median.

4) Marginalization = Score below the AAMAS-CO median and score below the AAMAS-EA median.

Sport fan identification. To measure identification with an ethnic player and a popular sport, the researcher used the Points of Attachment Index (PAI), originally developed for research within intercollegiate athletics (Trail, Robinson, Dick, \& Gillentine, 2003). The PAI was introduced as a comprehensive measure of an individual's different points of psychological attachment within a sport context (Kwon, Trail, \& Anderson, 2005). The PAI is composed of seven subscales with 21 items (each subscale has three items): (a) the team, (b) the players, (c) the coach, (d) the sport, (e) the level of sport (e.g., college basketball vs. professional basketball), (f) the university, and (g) the community. For the current study, the researcher adopted two subscales: attachment (identification) to the player (ethnic player) and attachment (identification) to the sport, but modified it to generate two types of sports [i.e., a popular sport in a native country and a popular sport in a host country (the U.S.)]. Given the differences of ethnic players and popular sports among the four ethnic groups, different ethnic players and popular sports were presented as examples depending on each of the four ethnic groups. More specifically, to measure identification with ethnic players and a popular sport for Chinese sample, two Chinese players (e.g., Yao Ming, Yi Jianlian) and basketball were chosen, respectively. In the same manner, three Japanese players (e.g., Ichiro Suzuki, Hideki Matsui, Daisuke Matsuzaka) and baseball were presented as examples for 
Japanese sample, Shin Soo Choo and baseball were presented as examples for Korean sample, and two Taiwanese athletes (e.g., Hong-Chih $\mathrm{Ku}$, Chein-Ming Whang) and baseball were presented as examples for Taiwanese sample, respectively. However, identification with a popular sport in the U.S. was measured by the same sport as an example across the four ethnic groups (American football in the study).

The PAI portion used in the current study for each sample (e.g., Chinese, Japanese, Korean, Taiwanese) included a total of 9 items on a 7-point Likert-type scale ranging from strongly disagree (1) to strongly agree (7). Although the sample for the current study is different from those in previous studies, those studies have found that the two subscales had good internal consistency: attachment to the player (Cronbach's alpha ranging from .81 to .87 ) and attachment to the sport (Cronbach's alpha ranging from.75 to .83) (Kwon et al., 2005; Robinson \& Trail, 2005; Robinson, Trail, \& Kwon, 2004; Trail et al., 2003) based on the suggested value of .70 (Nunnally \& Berstein, 1994). Further, construct validity of the two scales has been also supported in the previous studies mentioned earlier: attachment to the player (AVE value ranging from .63 to .72 ) and attachment to the sport (AVE value ranging from .54 to .83 ) meeting the minimum suggested value of .50 (Fornell \& Larcker, 1981).

Consumption behaviors. Measures for sport consumption behavior can be readily obtained from the existing literature. Yet, items utilized to measure sport consumption behavior vary considerably across studies. Thus, items believed to be most appropriate for the purposes of the current study and showing sound psychometric properties were initially selected and then reworded appropriate to the context of the current study. To measure participants' sport consumption behaviors, the researcher adopted a total of 13 
items from previous studies (Fink, Trail, \& Anderson, 2002b, 2008; Trail, Anderson, \& Fink, 2005). These items all were measured with a 7-point Likert-type scale ranging from strongly disagree (1) to strongly agree (7).

The current study measured three different types of sport consumption behaviors: (a) consumption behavior for a team with the ethnic player, (b) consumption behavior for a popular sport in a native country, and (c) consumption behavior for a popular sport in a host country. Similar to the measurement of identification, different ethnic players and popular sports were chosen as examples depending on each of the four ethnic groups when measuring sport consumption behavior for the two targets above (i.e., consumption behavior for a team with ethnic player, consumption behavior for a popular sport in a native country). However, consumption for a popular sport in the U.S.was measured by the same sport as an example across the four ethnic groups (American football in the study).

For consumption behavior for a team with the ethnic player, the researcher adopted five items designed to measure various forms of sport consumption behavior, including attendance at sporting events, merchandise consumption, and media consumption via TV, radio, and, or the Internet. Specifically, three items from Trail et al.'s (2005) study and two items from Fink et al.'s (2002) study were chosen. Since the three items from Trail et al.'s study were part of 4-item conative loyalty scale, internal consistency reliability and construct validity for the three items were not available. The 4 -item conative loyalty scale, however, had good internal consistency and construct validity (Cronbach's alpha $=.84$; $\mathrm{AVE}=.59)($ Trail et al.). Likewise, internal consistency reliability and construct validity for the two items from Fink et al.'s study were not available since the two items were also 
a subset of a 3 -item media consumption scale. The media consumption scale has shown good psychometric properties, however (Cronbach's alpha $=.87 ; \mathrm{AVE}=.70)$ (Fink et al.).

Due to the fact that consumption behavior for a popular sport in a native country cannot be measured by attendance, this consumption behavior was measured by three items closely related to sport media, particularly Internet (e.g., searching for and gathering information through the Internet), and merchandise. Specifically, two items from Fink et al.'s (2002) media consumption scale and two items from Trail et al.'s (2005) a 4-item conative loyalty scale were adopted.

Finally, consumption behavior for a popular sport in a host country (the U.S.) was measured by five items designed to measure various forms of sport consumption behavior, including attendance at sporting events, merchandise consumption, and media consumption through TV, radio, or the Internet. The five items were adopted from the same items used in measuring consumption behavior for a team with the ethnic player (Fink et al., 2002; Trail et al., 2005) and reworded to be appropriate to the consumption behavior for a popular sport in the U.S.

Demographics. Items measuring demographic characteristics of participants were included in the questionnaire. The items measured gender, age, ethnicity (Chinese, Japanese, Korean, Taiwanese), generational status, household income, marital status, educational level, immigration status, and length of residency in the U.S.

\section{Item Review}

Content validity was established through a panel of experts who reviewed items prior to administering the survey. The reviewers consisted of three experts who are knowledgeable about sport consumption behavior research. The experts were asked to 
review all survey items to evaluate whether their content is suitable for measuring the intended constructs. The panel's comments and recommendations were incorporated into the final version of the instruments.

\section{Back Translation}

For the participants who are not familiar with reading English, four bilingual graduate students corresponding to four country languages (one for each language) first translated the original English version of questionnaires into Chinese, Korean, Japanese, and Taiwanese, respectively. Four other bilingual graduate students then translated each of the four language version questionnaires back into English. The back-translation process minimizes the discrepancies and tests the equivalence between the original English version of questionnaires and each of the four different language questionnaires (Brislin, 1970). The comparison between the original English questionnaires and each of the four back-translated questionnaires led to the conclusion that the two forms of questionnaires were conceptually equivalent.

When the survey questionnaires were administered in the present study, the English version of questionnaires was provided, along with different language versions based upon types of participants (Chinese, Korean, Japanese, and Taiwanese). A series of oneway ANOVAs were conducted to determine whether there were statistically significant differences in the main variables involving the proposed three models between respondents with paper survey and those with online survey. The two versions of complete survey used for Chinese sample as an example were shown in Appendix C

\section{Pretesting}


Pretesting was conducted before the main study in order to test the reliability, validity, and the appropriateness of the instruments (Groves et al., 2004). The researcher followed several steps for pretesting suggested by Dillman (2007). First, all of the items in the survey were reviewed by the panel of experts who are knowledgeable about research method and design and sport consumption behavior. This occurred through the process of establishing content validity. Second, the survey revised by the panel of experts was distributed to eight Asian undergraduate and graduate students from the four countries (two participants from each of the four countries) at a large unban university in the Southeastern U.S. They were asked to provide comments with respect to the survey items' understandability, vagueness, redundancy, and its completion time. Comments provided by the participants in this process were incorporated into the next process of pretesting.

The last pretest step was to do a pilot study for Asian populations (i.e., Japanese, Chinese, Korean, and Taiwanese) living in a large unban city. According to Dillman (2007), approximately 100 to 200 respondents are generally required for a pilot study. Participants for the pilot study were recruited from Asian student associations at a large university and an Asian cultural center in the Southeastern U.S. via online survey. The participants for the pilot study were not participants for the main study.

\section{Data Analysis Procedures}

Data analysis was performed in three stages. First, descriptive statistics for the variables used in this study were calculated. Second, three separate measurement models corresponding to three proposed models for the current study were tested. Following the measurement models were the assessment of three separate structural models. This 
procedure using structural equation modeling (SEM) allowed for an assessment of the overall model fit and the hypothesized relationships proposed in each of the three sport consumption models for the current study (see Figure 1-1, 1-2, 1-3). The three structural models included ethnic identity, acculturation, identifications with an ethnic player and sports (a popular sport in a native country and a popular sport in a host country), and three sport consumption behaviors for the respective identification. Finally, multivariate analysis of covariances (MANCOVAs) were performed to analyze the impact of acculturation strategies on three types of identifications (IED, IPSNC, and IPSHC) and three types of sport consumption behaviors (CTEP, CPSNC, and CPSHC).

It is important to note that data analyses using both SEM and MANCOVAs were performed on the four subgroup samples (China, Japan, Korean, Taiwan) as one whole group rather than separate subgroups.

\section{Descriptive Statistics}

Various descriptive statistics were obtained using SPSS 19.0 to describe the basic characteristics of the data in this study. Measures of central tendency (e. g., mean, median) and measures of variability (e. g., standard deviation etc.) were calculated.

\section{Measurement Model}

Three separate confirmatory factor analyses (CFAs) were conducted in the three proposed models to evaluate the measurement models using Amos 19.0. More specifically, the first CFA was to test a measurement model specifying the relationships of the observed indicators (items) to the three latent constructs [(ethnic identity, acculturation, identification with an ethnic player (IEP), and consumption for a team with the ethnic player (CTEP) ]. The second and third CFAs were to assess the remaining two 
measurement models involving the same constructs as the first CFA, with exception of two constructs: identification with a popular sport in a native country (IPSNC) and consumption behavior for the sport (CPSNC) (the second CFA) and identification with a popular sport in a host country (IPSNC) and consumption for the sport (CPSHC) (the third CFA).

Internal consistency reliability coefficients (Cronbach's alpha coefficients) and Average Variance Extracted (AVE) values were calculated to evaluate internal consistency and convergent validity of the scores from the scales to be used in this study, respectively. When examining internal consistency for social science subscales, Cronbach's alpha values greater than .70 are assumed to be adequate (Nunnally \& Bernstein, 1994), while AVE values above .50 are adequate for convergent validity (Fornell \& Lacker, 1981; Hair et al., 1998). The discriminant validity was established through correlation coefficients among constructs. Kline (2005) suggested that once correlation coefficients between constructs are less than .85 , it indicates discriminant validity for the constructs. Furthermore, the discriminant validity can be established using AVE. If the AVE for each construct is greater than the squared correlations between one construct and any others, it provides the evidence of discriminant validity (Fornell \& Lacker).

Goodness of fit indices used to evaluate overall fit of the proposed models in the current study included the comparative fit index (CFI), Tucker-Lewis Index (TLI), and the standardized root-mean-square residual (SRMR) suggested by several scholars (Browne \& Cudeck, 1992;Hu \& Bentler, 1999; Kline, 2005). However, Browne and Cudeck (1992) suggested that the root-mean-square error of approximation (RMSEA) 
reduced problems with model fit that are not addressed by chi-square statistics.

Therefore, this study will also include RMSEA as well as chi-square value divided by the degrees of freedom as a frame of reference. CFI and TLI values greater than .95 are indicative of good-fitting models, the values between .90 and .95 are acceptable fit, while SRMR values of .08 or less are generally desired (Browne \& Cudeck, 1992; Hu \& Bentler, 1999). RMSEA values of less than .05 indicate good fit, values of .08 or less would indicate reasonable fit and values higher than .10 indicate poor fit (Brown \& Cudeck, 1992). Hu and Bentler suggested that values less than .06 should be considered to indicate that a model has a good fit.

\section{Structural Model}

To test the proposed hypothesized relationships in three models (see Chapter I), three separate structural regression models, incorporating ethnic identity, acculturation, identification with an ethnic player and sports, and three sport consumption behaviors for the respective identification, were conducted using Amos 19.0. Each of the three structural regression models were estimated using each scale item as an indicator of its associated latent construct. Since a structural regression model is a more comprehensive and powerful way to examine the relationships among the latent constructs above, the current study tested the three proposed models with the structural regression models. Furthermore, the use of structural regression model to simultaneously test the relationship among the latent constructs provides "a more satisfactory approach to construct validation"(Diamantopoulos \& Winklhofer, 2001, p. 272).

\section{MANCOVA}


MANCOVA is an extension of analysis of covariance (ANCOVA), used in studies with more than one dependent variable (Stevenson, 2005). The current study used the covariate (length of residence in the host country) to reduce the variability in the dependent variables by removing for the dependent variable variances predicted from the covariate.

To investigate the impact of acculturation strategies on identifications with an ethnic player and sports and sport consumption behaviors for the respective identification, three separate MANCOVAs were performed. For the first MANCOVA, the researcher attempted to examine whether there were significant differences in IEP and CTEP based on four different acculturation strategies (integration, assimilation, separation, and marginalization), while controlling for the effect of length of residence in the U.S. For this analysis, acculturation strategies served as independent variables and IEP and CTEP served as dependent variables. Length of residence in the U.S. served as a covariate. The second MANCOVA was performed to examine whether a significant difference exists in IPSNC and CPSNC based upon the four different acculturation strategies, while controlling for the effect of length of residence in the U.S. For this analysis, acculturation strategies served as an independent variable and IPSNC and CPSNC served as dependent variables. Length of residence in the U.S. served as a covariate.

The third MANCOVA was performed to examine whether significant difference exists in IPSHC (i.e., American football) and CPSHC based upon the four different acculturation strategies, while controlling for the effect of length of residence in the U.S. For this analysis, acculturation strategies served as an independent variable and IPSHC 
and CPSHC served as dependent variables. Length of residence in the U.S. also served as a covariate.

In MANOVA or MANCOVA, dependent variables should be conceptually correlated with each other (Harrison, 2011). Since research on sport consumption behaviors revealed that fan identification is theoretically and empirically correlated with sport consumption behavior, it is scientifically sound to employ MANCOVAs to examine the impacts of acculturation strategies on fan identification and sport consumption behavior.

When conducting MANCOVAs, several assumptions need to be met (Stevens, 2005). These assumptions included normal distribution of dependent variables, equality of variance-covariance matrices, a linear relationship between dependent variables and the covariate (controlling variable), and homogeneity of regression slopes, meaning that the slopes of the regression lines for each level of an independent variable are equal. All of these assumption checks were occurred prior to conducting MANCOVAs. 


\section{CHAPTER IV}

\section{RESULTS}

The first purpose of the current study was to develop and test three Sport Consumption Models for the Asian population living in the U.S. using ethnic identity and acculturation. To fulfill the above purpose, the study measured ethnic identity, acculturation, three types of attachments [e.g., identification with an ethnic player (IEP), identification with a popular sport in a native country (IPSNC), identification with a popular sport in a host country (IPSHC)], and three sport consumption behaviors for the respective attachment (identification) [e.g., consumption for a team with ethnic player (CTEP), consumption for a popular sport in a native country (CPSNC), and consumption for a popular sport in a host country (CPSHC)]. Three confirmative factor analyses (CFAs) and three structural regression analyses were conducted to test the three proposed models presented in Figures 1-1, 1-2, and 1-3 (see Chapter I). In addition to this purpose, the second purpose of the study was to examine the impact of four acculturation strategies (e.g., integration, assimilation, marginalization, separation) on the three attachments and consumption behaviors. Three separate MANCOVAs were employed to achieve the second purpose.

This chapter includes the following seven sections: (a) pretests, (b) sample characteristics, (c) preliminary analysis, (d) descriptive statistics for the main variables, (e) measurement models, (f) structural models, and (g) results of MANCOVAs. Detailed results of the current study are presented below. 


\section{Pretests}

\section{Panel of Expert}

Prior to the main study, the current study utilized several steps for pretesting suggested by Dillman (2007). In order to establish content validity, the panel of experts was asked to review the appropriateness of the survey instrument. Two experts indicated that since the term, "culture of origin," in all 15 items of the AAMAS-CO might confuse the respondents, it needed to be replaced with specific ethnic groups based on the survey populations (e.g., Chinese, Japanese, Korean, Taiwanese) to clarify the meaning of "culture of origin". In addition, one expert made a comment on whether one's own selection of popular sport in a native and host country, or of a specific ethnic player competing on a North American professional team is different from the proposed example popular sports and ethnic players in the survey questionnaire. Based on this comment, the researcher added three open-ended questions to see if there is consistency between respondents' selection of popular sports/ethnic players and those in the survey questionnaire: (a) "Who is your favorite (name of ethnic group) player playing for an American professional team?", (b) What do you think is the most popular sport in (name of original country)?", and (c) What do you think is the most popular sport in America? Other than these suggestions, the panel of experts agreed that all items were measuring what they're supposed to measure.

\section{Field Test}

Following the review by the panel of experts, a field test was conducted to ensure the revised survey items' understandability, ambiguity, redundancy, and the amount of time needed to complete the survey. Eight Asian undergraduate and graduate students 
from the four countries at a large Midwestern university (two students from each of the four countries) completed the survey, and then filled out a short comment form (see Appendix D). The respondents were asked to read the survey thoroughly, and answered each question on the comment form. Most respondents indicated the survey was easy to understand, the directions were easy to follow, and items on the survey were appropriate to achieve the study's purposes.

\section{Pilot Study}

A pilot study was conducted to determine the internal consistency reliability of all subscale scores. Asian students/faculty members from the four target countries at a Midwestern university were asked to complete the survey online. The participants were asked to choose between an English version and their native langue version. A total of 64 participants took part in the pilot study. The sample consisted of 50\% male $(n=32)$ and $50 \%$ female $(n=32)$. The majority of the sample was Chinese $(n=37,57.8 \%)$ with the remainder of the sample as follows: Japanese $(n=8,12.5 \%)$, Korean $(n=10,15.6 \%)$, and Taiwanese $(n=9,14.1 \%)$. The average period living in the U.S. was 6.68 years and the average age of participants was 28.67 years old. Over $80 \%$ of the participants $(n=52)$ used the English version survey.

Cronbach's alpha coefficients ranged from .77 (acculturation) to .91 (CPSNC). Since all Cronbach's alpha coefficients exceeded the recommended cutoff of .70 (Nunnally \& Bernstein, 1994), the survey instrument was deemed reliable. A complete listing of the Cronbach's alpha coefficients for all subscales of the pilot study can be found in Table 2.

Table 2. 
Cronbach's Alpha Coefficients ( $\alpha$ ) for All Subscales Included in the Survey

Questionnaire for the Pilot Study

\begin{tabular}{lcc}
\hline Name of Subscale & $\begin{array}{c}\text { Numbers } \\
\text { of Items }\end{array}$ & Cronbach's Alpha \\
\hline Ethnic Identity (EID) & 3 & .89 \\
$\begin{array}{l}\text { Acculturation (ACC) } \\
\text { Identification with Player (IEP) }\end{array}$ & 6 & .77 \\
$\begin{array}{l}\text { Identification with Popular Sport } \\
\text { in a Native Country (IPSNC) }\end{array}$ & 3 & .84 \\
$\begin{array}{l}\text { Identification with Popular Sport } \\
\text { In a Host Country (IPSHC) }\end{array}$ & 3 & .87 \\
$\begin{array}{l}\text { Consumption for a Team } \\
\text { with Ethnic Player (CTEP) }\end{array}$ & 5 & .86 \\
$\begin{array}{l}\text { Consumption for a Popular Sport } \\
\text { in a Native Country (CPSNC) }\end{array}$ & 4 & .91 \\
$\begin{array}{l}\text { Consumption for a Popular Sport in a Host } \\
\text { Country (CPSHC) }\end{array}$ & 5 & .90 \\
\hline
\end{tabular}

\section{Sample Characteristics}

During November 2011, a total of 303 surveys for the main study were collected from the four ethnic groups living in the following four cities: (a) Houston for Chinese, (b) Seattle for Japanese, (c) Cleveland for Korean, and (c) L.A. for Taiwanese. Two hundred participants (66\%) filled out the survey in English, while 103 participants (34\%) filled out the survey in a native language. A total of 239 questionnaires $(78.9 \%)$ were collected via online survey, and the other 64 surveys $(21.1 \%)$ were collected through paper survey. After reviewing the surveys, 12 surveys were eliminated due to incomplete or unusable data. The final sample size for data analysis was 291. Based on the aggregated number of 
members at the seven organizations (948), this sample size exceeded the minimum threshold of 278 participants using a $95 \%$ confidence level with sampling error $\pm 5 \%$ (Salant \& Dillman, 1994). In addition, Kline (2005) suggested the sample size in SEM should be greater than 20 times of the free parameters. Each of the three proposed models for the current study has 10 freely estimated parameters, equating to a minimum of 200 participants. Therefore, the final sample of 291 for the current study exceeded this minimum threshold.

The sample consisted of 88 Chinese (30.2\%), 55 Japanese (18.9\%), 86 Korean (29.6\%), and 62 Taiwanese (21.3\%). For gender breakdown, 157 were male $(54 \%)$ and 134 were female $(46 \%)$. With regard to age, the average age of the sample was 30.30 (SD $=8.23$ years). With regard to the length of residency in the U.S., the average living period in the U.S. was 9.52 ( $S D=8.45$ years). All participants were asked to indicate what they thought was the most popular sport in a native country and the U.S (host country), and who they thought was the most favorite ethnic player playing or recently played for an American professional team. The majority of the participants reported the same popular sports in a native country $(n=192 ; 65.9 \%)$ and host country $(n=195 ; 67 \%)$ as those presented as examples in the survey, and the same ethnic players $(n=193 ; 66.3 \%)$ as those presented in the survey.

Other demographic information collected in the current study included (a) marital status, (b) educational level, (c) generation status, (d) U.S. immigration status, and (e) annual household income level. The frequency distribution of marital status indicated that $158(54.3 \%)$ were single (never married), $110(37.8 \%)$ were married, $18(6.2 \%)$ were divorced or separated, and $3(1.0 \%)$ were widowed. Seven participants did not indicate 
their marital status. For educational level, 35.7\% $(n=104)$ of the sample had bachelor's degree, $25.8 \%(n=75)$ had master's degree, $22.4 \%(n=65)$ had high school diploma, $13.4 \%(n=39)$ had doctoral degree, and $2.4 \%(n=7)$ indicated other. One participant failed to respond to this question. Regarding participants' generation status, approximately $50.2 \%(n=146)$ of participants were temporarily staying in the U.S., 66 $(22.7 \%)$ were 1 st generation (I was born and grew up in Asia. I live in America as a resident), 57 (19.6\%) were 1.5 generation (I was born in an Asian country. When I was young, I imigrated to America and grew up here), and the remaining participants $(n=5$, 7.8\%) considered themselves as either 2nd (My parents are the first generation of immigrants) or 3rd generations (My parents are the second generation of immigrants).

With regard to U.S. immigration status, 122 participants (41.9\%) were held a student visa, 73 ( $n=25.1 \%)$ were U.S. citizens, $64(22.0 \%)$ held green card (permanent resident), $20(6.9 \%)$ held working visa, and nine participants $(3.1 \%)$ indicated other. Three participants did not indicate their U.S. immigration status.

For annual household income, participants reporting an annual household income of less than $\$ 19,000$ comprised $34.0 \%(n=99)$ of the total sample, which was the largest group. Those reporting annual household income in the $\$ 20,000-\$ 39,999$ range comprised the second largest group $(n=78 ; 26.8 \%)$, followed by $\$ 40,000-\$ 59,999$ range $(n=30 ; 10.3 \%), \$ 60,000-\$ 79,999$ range $(n=25 ; 8.6 \%)$, more than $\$ 100,000(n=$ $29 ; 10.0 \%)$, and $\$ 80,000-\$ 99,999$ range $(n=20 ; 6.9 \%)$. Ten participants $(3.4 \%)$ did not response the annual household income question. Complete demographic characteristics for the current study were presented in Table 3 .

Table 3. 
Summary of Demographic Characteristics of the Study $(N=291)$

\begin{tabular}{|c|c|c|c|}
\hline Variable & Category & Frequency $(\mathrm{n})$ & Percentage (\%) \\
\hline \multirow{4}{*}{ Ethnicity } & Chinese & 88 & $30.2 \%$ \\
\hline & Japanese & 55 & $18.9 \%$ \\
\hline & Korean & 86 & $29.6 \%$ \\
\hline & Taiwanese & 62 & $21.3 \%$ \\
\hline \multirow[t]{2}{*}{ Gender } & Male & 157 & $54 \%$ \\
\hline & Female & 134 & $46 \%$ \\
\hline \multirow[t]{4}{*}{ Marital Status } & Single(Never married) & 158 & $54.3 \%$ \\
\hline & Married & 110 & $37.8 \%$ \\
\hline & Divorced or Separated & 18 & $6.2 \%$ \\
\hline & Widowed & 3 & $1.0 \%$ \\
\hline \multirow[t]{5}{*}{ Educational Level } & High School & 65 & $22.3 \%$ \\
\hline & Bachelor's Degree & 104 & $35.7 \%$ \\
\hline & Master's Degree & 75 & $25.8 \%$ \\
\hline & Doctoral Degree & 39 & $13.4 \%$ \\
\hline & Other & 7 & $2.4 \%$ \\
\hline \multirow[t]{5}{*}{ Generation Status } & Asian & 146 & $50.2 \%$ \\
\hline & 1st Generation & 66 & $22.7 \%$ \\
\hline & One and a half Generation & 57 & $19.6 \%$ \\
\hline & 2nd Generation & 20 & $6.9 \%$ \\
\hline & 3rd Generation & 2 & $.7 \%$ \\
\hline U.S. Immigration & U.S. Citizenship & 73 & $25.1 \%$ \\
\hline \multirow[t]{4}{*}{ Status } & $\begin{array}{l}\text { Green Card (Permanent } \\
\text { Resident) }\end{array}$ & 64 & $22.0 \%$ \\
\hline & Student Visa & 122 & $41.9 \%$ \\
\hline & Working Visa & 20 & $6.9 \%$ \\
\hline & Other & 9 & $3.1 \%$ \\
\hline Annual Household & Less than $\$ 19,999$ & 99 & $35.1 \%$ \\
\hline \multirow[t]{5}{*}{ Income } & $\$ 20,000-\$ 39,999$ & 78 & $26.8 \%$ \\
\hline & $\$ 40,000-\$ 59,999$ & 30 & $10.3 \%$ \\
\hline & $\$ 60,000-\$ 79,000$ & 25 & $8.6 \%$ \\
\hline & $\$ 80,000-\$ 99,000$ & 20 & $6.9 \%$ \\
\hline & $\$ 100,000+$ & 29 & $10.0 \%$ \\
\hline \multirow[t]{3}{*}{ Favorite Ethnic Player } & Matching in the Survey & 193 & $66.3 \%$ \\
\hline & Non-matching in the Survey & 42 & $15.5 \%$ \\
\hline & No Response or Not Sure & 56 & $19.2 \%$ \\
\hline
\end{tabular}




$\begin{array}{llcc}\text { Popular Sport in a } & \text { Matching in the Survey } & 192 & 66.0 \% \\ \text { Native Country } & \text { Non-matching in the Survey } & 41 & 14.0 \% \\ & \text { No Response or Not Sure } & 58 & 19.9 \% \\ \text { Popular Sport in the } & \text { Matching in the Survey } & 195 & 67.0 \% \\ \text { U.S. (Host Country) } & \text { Non-matching in the Survey } & 75 & 25.8 \\ & \text { No Response or Not Sure } & 21 & 7.2 \%\end{array}$

Note. The average age of the sample $=30.30$ years and the average length of residency in the U.S. $=9.52$ years.

\section{Preliminary Analysis}

As noted in Chapter III, data were collected using the combination of online and paper surveys. In each of the two data collection methods, participants were also given the choice between completing the survey written in English and one written in a native language. For this reason, preliminary analyses using a series of one-way ANOVAs were completed to determine whether significant differences existed in the main variables involving in the proposed three sport consumption models (i.e., EID, ACC, IEP, IPSNC, IPSHC, CTEP, CPSNC, and CPSHC) between data collected via paper versus online surveys and data collected via English version versus a native language version surveys.

In addition, it was possible that collecting data from Asian student associations and community centers may have given rise to the increment of skewness for ethnic identity and acculturation variables. In other words, the scores on ethnic identity were likely to be high (negative skewness: scores weigh heavily to the right side of the distribution), whereas those on acculturation might be low (positive skewness: scores weigh heavily to the left side of the distribution). Due to this possibility, the researcher also checked the magnitude of skewness for the two variables (ethnic identity and acculturation) in the preliminary data analysis. 


\section{Paper versus Online Surveys}

Of the 291 usable surveys, 227 were completed through online survey, whereas 64 participants completed the paper survey. The results of one-way ANOVAs indicated no statistically significant differences in all the main variables between online and paper surveys. Specific results were as follows: (a) ethnic identity $F(1,289)=.166, p=.684$ for ethnic identity; (b) $F(1,289)=1.87, p=.173$ for acculturation; (c) $F(1,289)=.167, p$ $=.683$ for identification with an ethnic player (IEP); (d) $F(1,289)=1.250, p=.264$ for consumption for a team with ethnic player (CTEP); (e) $F(1,289)=2.628, p=.106$ for identification with a popular sport in a native country (IPSNC); (f) $F(1,289)=.987, p$ $=.321$ for consumption for a popular sport in a native country $(\mathrm{CPSNC}) ;(\mathrm{g}) F(1,289)$ $=.712, p=.599$ for identification with a popular sport in a host country (IPSHC); and (h) $F(1,289)=.178, p=.674$ for consumption for a popular sport in a host country (CPSHC). Since no differences were found between online and paper surveys, it does not need to be controlled.

\section{English versus Native Language Respondents}

Among 291 participants, 188 completed the survey in English, whereas 103 completed the survey in one of the four native languages (Chinese, Japanese, Korean, and Taiwanese). The results of one-way ANOVAs revealed no statistically significant differences in seven of the eight variables between English and native version surveys, with the exception of the acculturation variable. Specific results were as follows: (a) ethnic identity $F(1,289)=.460, p=.498$ for ethnic identity; (b) $F(1,289)=.177, p=.674$ for IEP; (c) $F(1,289)=2.823, p=.094$ for CTEP; (d) $F(1,289)=.185, p=.667$ for IPSNC; (e) $F(1,289)=.054, p=.817$ for CPSNC; (f) $F(1,289)=.067, p=.599$ for 
IPSHC; and $(\mathrm{g}) F(1,289)=.486, p=.486$ for CPSHC. However, the only statistically significant difference in the levels of acculturation was observed between respondents completing the English survey and those completing the native language survey, $F(1,289)$ $=7.462, p=.007$. More specifically, respondents completing the English survey had higher levels of acculturation $(M=4.41, S D=.81)$ than those completing the native language survey $(M=4.15, S D=.74)$. Considering language is one of the key domains to measure for the acculturation variable (see Chapter III), this finding was understandable because respondents completing the English survey would be more likely to adapt to the U.S. than their counterparts. However, extra caution should be paid when interpreting the results.

\section{Skewness Check for Ethnic Identity and Acculturation}

To check the magnitude of skewness for the two variables, the researcher utilized absolute skewness values suggested by Chou and Bentler (1995). The skewness values of ethnic identity and acculturation were -.623 and .000 , respectively. These absolute values did not indicate strong departures from normality because they were less than 3.0 suggested by Chou and Bentler (1995). Therefore, the scores on the two variables represented a satisfactory normal distribution, although data collection occurred in Asian student association and community/cultural centers.

\section{Descriptive Statistics for Main Variables}

As noted above, the main variables involved in the three proposed models (see Chapter I) were: (a) ethnic identity, (b) acculturation, (c) identification with an ethnic player (IEP), (d) identification with a popular sport in a native country (IPSNC), (e) identification with a popular sport in a host country (IPSHC), (f) consumption for a team 
with an ethnic player (CTEP), (g) consumption for a popular sport in a native country (CPSNC), and (h) consumption for a popular sport in a host country (CPSHC). Each of the eight constructs was measured on a 7-point Likert-type scale, ranging from $1=$ strongly disagree to $7=$ strongly agree, except for the acculturation construct. The acculturation construct was also measured on a 7-point Likert type scale, but anchored with $1=$ Not at $A l l$ to $7=$ Very Much. The means, standard deviations, and skewness and kurtosis values for the eight main variables involved in the three proposed models are presented in Table 4. Detailed explanations for descriptive statistics for each of the key variables follow below.

Ethnic identity was measured with three items adopted from the MEIM-R (Phinney $\&$ Ong, 2007). For the entire sample, the overall mean for ethnic identity was 5.05 ( $S D=$ 1.48). The reported skewness and kurtosis values were -.623 and -.321 respectively, which was below the cutoff of 3.0. For acculturation, it had a mean of $4.32(S D=.79)$, with skewness of .000 and kurtosis of .115 .

With regard to the three different identifications (points of attachment), IEP had a mean of $3.65(S D=1.72)$, IPSNC had a mean of $2.96(S D=1.66)$, and IPSHC had a mean of $2.71(S D=1.60)$. The mean scores for all the three identification variables were normally distributed based on skewness values (ranged from .194 to .835) and kurtosis values (ranged from -.238 to -.993 ).

As for the three consumption variables, CTEP had a mean of $2.71(S D=1.39)$, CPSNC had a mean of $2.12(S D=1.27)$, and CPSHC had a mean of $2.47(S D=1.39)$. All three mean scores regarding consumption variables were also normally distributed based 
on skewness values (ranged from 1.22 to .693 ) and kurtosis values (ranged from -.332 to .946$)$.

Table 4.

Means, Standard Deviations, Skewness, and Kurtosis for the Main Variables

\begin{tabular}{lcccc}
\hline Variables & Mean & SD & Skewness & Kurtosis \\
Ethnic Identity (EID) & 5.05 & 1.48 & -.623 & -.321 \\
Acculturation (ACC) & 4.32 & .79 & .000 & .115 \\
Identification with Ethnic Player (IEP) & 3.65 & 1.72 & .194 & -.993 \\
$\begin{array}{l}\text { Identification with Popular Sport in a } \\
\text { Native Country (IPSNC) }\end{array}$ & 2.96 & 1.66 & .616 & -.558 \\
$\begin{array}{l}\text { Identification with Popular Sport in a } \\
\text { Host Country (IPSHC) }\end{array}$ & 2.71 & 1.60 & .836 & -.238 \\
$\begin{array}{l}\text { Consumption for a Team with Ethnic } \\
\text { Player (CTEP) }\end{array}$ & 2.71 & 1.39 & .693 & -.332 \\
$\begin{array}{l}\text { Consumption for a Popular Sport in a } \\
\text { Native Country (CPSNC) }\end{array}$ & 2.12 & 1.27 & 1.22 & .946 \\
$\begin{array}{l}\text { Consumption for a Popular Sport in a } \\
\text { Host Country (CPSHC) }\end{array}$ & 2.47 & 1.39 & 1.16 & .716 \\
\hline
\end{tabular}

\section{Measurement Models}

Confirmatory factor analyses (CFAs) were first conducted to test three measurement models for the respective proposed models (Figures 1-1, 1-2, 1-3) before testing structural relationships among the constructs in the three models. The specific results of the measurement models are described below.

\section{Ethnic Player Model}


This measurement model specified four latent factors, consisting of 17 items (ethnic identity: three items; acculturation: six items; IEP: three items; and CTEP: five items), to be correlated with each other. The results of CFA indicated this model did not appropriately fit to the current data based upon several fit indices $\left(x^{2} / d f=550.191 / 113=\right.$ $4.869, \mathrm{RMSEA}=.116, \mathrm{TLI}=.879, \mathrm{CFI}=.900, \mathrm{SRMR}=.068)$. Especially, the RMSEA was far below what is recommended for a good fit. Theoretical relevance, empirical criteria (e.g., factor loadings, modification index (MI), standardized residuals), and parsimoniousness of the model were collectively considered in order to improve the model fit. Factor loadings for the initial CFA were presented in Table 5. Factor loading of item 6 (How negative do you feel about people of American culture?) on acculturation ( $\beta$ $=.074, p=.230$ ) was not statistically significant, so that item 6 was deleted. The main reason this item was not significant was thought to be due to negative wording. In addition to item 6 on acculturation, item 2 (How much do you interact and associate with people from American culture?) on acculturation was deleted because of the factor loading $(\beta=.458)$ that was lower than the recommended value of .50 (Hair et al., 1998). This item deletion was also supported by standardized residuals indicating discrepancies between "the implied theoretical covariance matrix and the observed covariance matrix that are statistically significant and reflect a potential source of misfit" (Netermeyer, Bearden, \& Sharma, 2003, p. 154).

According to Joreskog and Sorbom (1998), an observed value greater than 2.58 Table 5. Factor Loadings of the 17 items for Ethnic Player Measurement Model

\begin{tabular}{lll}
\hline Name of Factor & Item & Factor Loading
\end{tabular}




\begin{tabular}{lll}
\hline \multirow{2}{*}{ Ethnic Identity } & Item 1 & .930 \\
& Item 2 & .971 \\
& Item 3 & .882 \\
\hline \multirow{2}{*}{ Acculturation } & Item 1 & .821 \\
& Item 2 & .458 \\
& Item 3 & .916 \\
& Item 4 & .597 \\
\hline Identification with an & Item 5 & .531 \\
Ethnic Player (IEP) & Item 6 & .074 \\
\hline - & Item 1 & .877 \\
Consumption for a Team & Item 2 & .966 \\
with Ethnic Player (CTEP) & Item 3 & .956 \\
\hline & Item 1 & .850 \\
& Item 2 & .941 \\
& Item 3 & .933 \\
\hline
\end{tabular}

indicates statistically significant discrepancy of covariance between observed variables (items). Item 6 on acculturation had two standardized residuals over the benchmark of 2.58 (Joreskog \& Sorbom, 1998). Thus, items 2 and 6 of acculturation factor were deleted in order to improve the model fit.

After deleting the two items (items 2 and 6 of acculturation), the second CFA was conducted with the 15 items of the measurement model. The results of the second CFA showed the revised measurement model was not still a good fitting model $\left(x^{2} / \mathrm{df}=\right.$ $447.172 / 84=5.323, \mathrm{RMSEA}=.122, \mathrm{TLI}=.892, \mathrm{CFI}=.914, \mathrm{SRMR}=.062)$. In order to 
improve the model fit, modification indices (MIs) were examined. The MI refers to "the statistical significance of an unspecified model relationship and represents the approximate reduction in $\chi^{2}$ that would be obtained by estimating the unspecified parameter of interest" (Netemeyer et al., 2003, p. 155). In the second CFA, the highest MI (175.830) was a correlated error between items 4 and 5 of consumption factor (CTEP). In addition, item 5 had the highest standardized residual (5.463). Based on these, item 5 of consumption factor (I buy the team's clothing from an American team that has a player from my native country) was eliminated. This item elimination was also supported by content redundancy with item 4 within the same factor (I purchase the team's merchandise from an American team that has a player from my native country).

After deleting item 5 of consumption factor, third CFA was done with 14 items of the measurement model. The results of the third CFA indicated that the measurement model showed good fit to the data $\left(x^{2} / \mathrm{df}=189.768 / 71=2.673, \mathrm{RMSEA}=.076(90 \%\right.$ confidence interval $=.06-08), \mathrm{TLI}=.960, \mathrm{CFI}=.969, \mathrm{SRMR}=.052)$. Given the adequate fit of the model, no further modification was necessary. The final measurement model for ethnic player had 14 observed variables (items) for 4 factors (ethnic identity: 3 items; acculturation: 4 items; IEP: 3 items; and CTEP: 4 items) (see Figure 5). 


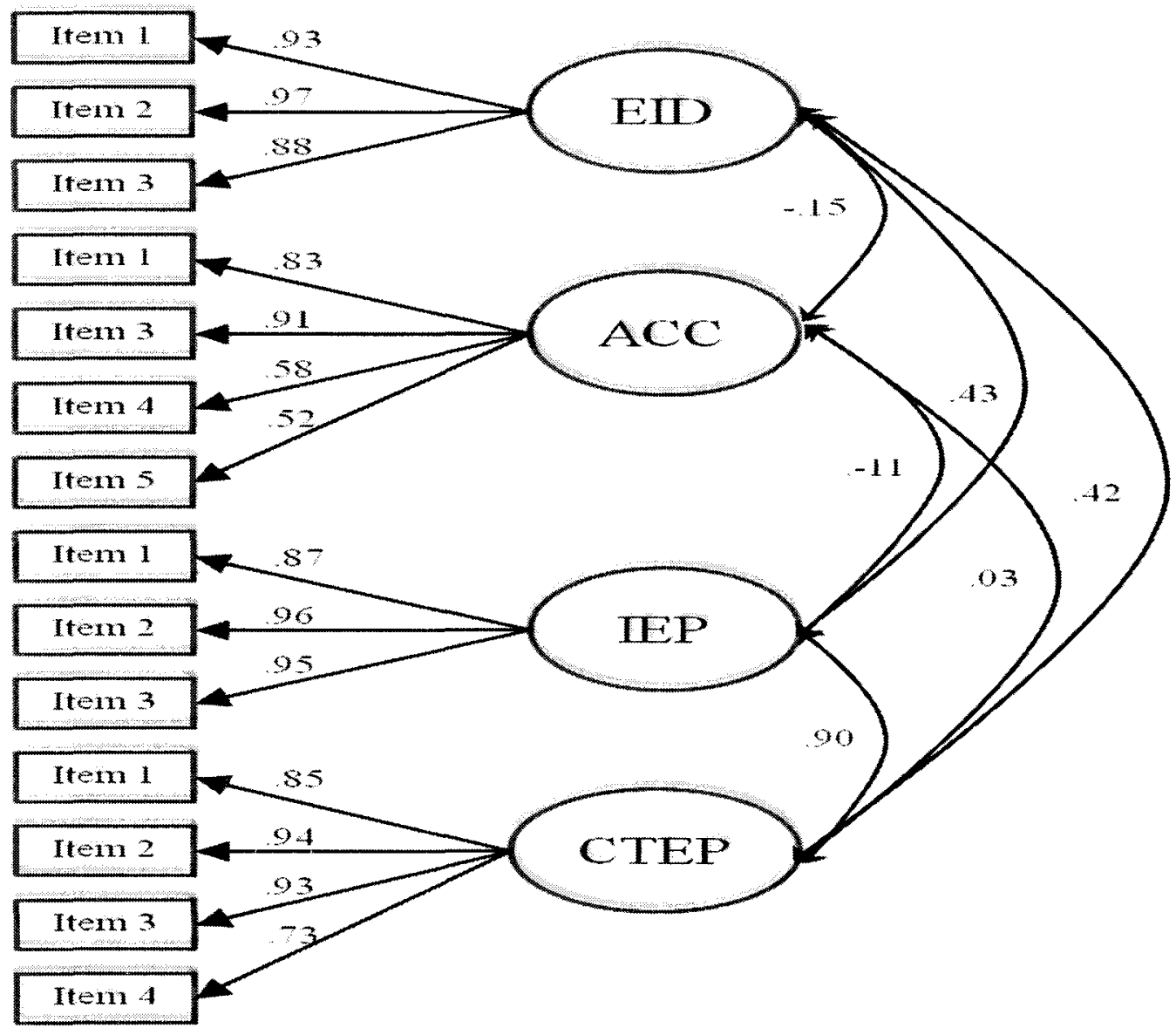

Figure 5. The Final Measurement Model for Ethnic Player. Note. EID = Ethnic Identity; $\mathrm{ACC}=$ Acculturation; $\mathrm{IEP}=$ Identification with an Ethnic Player; and CTEP = Consumption for a Team with an Ethnic Player.

Reliability and validity of the model. Factor loadings, Cronbach's alpha coefficients, and averaged extracted variance (AVE) for the final ethnic player measurement model are reported in Table 6 . The internal consistency reliability coefficients for all the factors were above the benchmark of .70 (Nunnally \& Berstein, 1994), ranging from .81 for acculturation to .95 for IEP. The AVE values for all the factors were also greater than the cutoff of .50 (Fornell \& Lacker, 1981), ranging from .52 for acculturation to .85 for both ethnic identity and IEP. All correlations among 
the four factors were less than .85 (Kline, 2005) with the exception of correlation between IEP and CTEP (see Table 7). Furthermore, the most AVE values were greater than the squared correlation between one factor and other factors. The squared correlation between IEP and CTEP $\left(r^{2}=.81\right)$, however, was greater than the AVE value of CTEP (.74). The above results indicated that the overall measurement model for ethnic player showed appropriate internal consistency reliability and construct validity (e.g., discriminant and convergent validity).

Table 6.

Factor Loadings ( $\beta$ ), Cronbach's Alpha Coefficients, and AVE for the Final Ethnic

Player Measurement Model

\begin{tabular}{|c|c|c|c|c|}
\hline Name of Factor & Item & $\beta$ & $\alpha$ & AVE \\
\hline \multirow[t]{4}{*}{ Ethnic Identity } & & & .94 & .85 \\
\hline & Item 1 & .93 & & \\
\hline & Item 2 & .97 & & \\
\hline & Item 3 & .88 & & \\
\hline \multirow[t]{5}{*}{ Acculturation } & & & .81 & .52 \\
\hline & Item 1 & .83 & & \\
\hline & Item 3 & .91 & & \\
\hline & Item 4 & .58 & & \\
\hline & Item 5 & .52 & & \\
\hline \multirow{4}{*}{$\begin{array}{l}\text { Identification with Ethnic } \\
\text { Player (IEP) }\end{array}$} & & & .95 & .85 \\
\hline & Item 1 & .87 & & \\
\hline & Item 2 & .96 & & \\
\hline & Item 3 & .95 & & \\
\hline \multirow{5}{*}{$\begin{array}{l}\text { Consumption for a Team with } \\
\text { Ethnic Player (CTEP) }\end{array}$} & & & .93 & .74 \\
\hline & Item 1 & 85 & & \\
\hline & Item 2 & .94 & & \\
\hline & Item 3 & .93 & & \\
\hline & Item 4 & .73 & & \\
\hline
\end{tabular}

Table 7. 
Correlations among All the Four Factors for Ethnic Player Model

\begin{tabular}{lcccc}
\hline Factor & 1 & 2 & 3 & 4 \\
\hline 1. Ethnic ID & 1.00 & & & \\
2. Acculturation & -.145 & 1.00 & & \\
3. IEP & .426 & -.113 & 1.00 & \\
4. CTEP & .417 & .033 & .901 & 1.00 \\
\hline
\end{tabular}

\section{Native Sport Model}

This measurement model specified four latent factors, consisting of 16 items (ethnic identity: three items; acculturation: six items; identification with a popular sport in a native country (IPSNC): three items; and consumption for a popular sport in a native country (CPSNC): four items), to be correlated with each other. The results of CFA indicated that this model did not appropriately fit to the current data based upon several fit indices $\left(x^{2} / d f=611.145 / 98=6.236, \mathrm{RMSEA}=.134, \mathrm{TLI}=.844, \mathrm{CFI}=.872, \mathrm{SRMR}\right.$ $=.082)$. Particularly, the RMSEA was far below what is recommended for a good fit. In order to improve the model fit, theoretical relevance, empirical criteria (e.g., factor loadings, modification index (MI), standardized residuals), and parsimoniousness of the model were collectively taken into account. Factor loadings for the initial CFA were presented in Table 8. Like ethnic player measurement model, factor loading of item 6 (how negatively do you feel about people of American culture?) on acculturation ( $\beta$ $=.079, p=.201)$ was not statistically significant, so item 6 was deleted. The nonsignificant result was probably due to the negative wording of this item. In addition to item 6 on acculturation, item 2 (How much do you interact and associate with people from American culture?) on the acculturation factor was deleted because of relatively low 
factor loading $(\beta=.462)$ that was lower than the recommended value of .50 (Hair et al., 1998). Further, since there were also two standardized residuals over the benchmark of 2.58 , the researcher deleted item 2 on acculturation factor.

Table 8 .

Factor Loadings $(\beta)$ of the 16 items for Native Sport Measurement Model

\begin{tabular}{|c|c|c|}
\hline Name of Factor & Item & Factor Loading \\
\hline \multirow{3}{*}{ Ethnic Identity } & Item 1 & .931 \\
\hline & Item 2 & .970 \\
\hline & Item 3 & .881 \\
\hline \multirow{6}{*}{ Acculturation } & Item 1 & .817 \\
\hline & Item 2 & .462 \\
\hline & Item 3 & .920 \\
\hline & Item 4 & .595 \\
\hline & Item 5 & .527 \\
\hline & Item 6 & .079 \\
\hline \multirow{3}{*}{ IPSNC } & Item 1 & .949 \\
\hline & Item 2 & .933 \\
\hline & Item 3 & .882 \\
\hline \multirow{4}{*}{ CPSNC } & Item 1 & .976 \\
\hline & Item 2 & .968 \\
\hline & Item 3 & .651 \\
\hline & Item 4 & .553 \\
\hline
\end{tabular}

The revised measurement model for native sport was tested after the deletion of items 2 and 6 on acculturation factor. The results of second CFA indicated that the 
revised measurement model did not still adequately fit to the data $\left(x^{2} / \mathrm{df}=510.342 / 71=\right.$ $7.188, \mathrm{RMSEA}=.146, \mathrm{TLI}=.855, \mathrm{CFI}=.887, \mathrm{SRMR}=.078)$. In order to improve the model fit, the MIs and standardized residuals were examined. In the second CFA, the highest MI (206.339) was a correlated error between items 3 and 4 of CPSNC factor. In addition, item 4 had the highest standardized residual (8.423). Based upon the MI and standardized residual, item 4 of CPSNC factor (I buy the clothing of a popular sport in my native country) was deleted. Besides empirical reasons, this item deletion was also theoretically supported because item 4 was very conceptually redundant with item 3 within the same factor (I purchase the merchandise of a popular sport in my native country).

After deleting item 4 of consumption factor, the third CFA was conducted with the remaining 13 items. The third CFA results indicated that the measurement model showed a good fit to the data $\left(x^{2} / \mathrm{df}=151.063 / 59=2.560, \mathrm{RMSEA}=.073(90 \%\right.$ confidence interval $=.05-08), \mathrm{TLI}=.965, \mathrm{CFI}=.973 \mathrm{SRMR}=.058)$. Considering the adequate fit of the model, no further modification was needed. The final measurement model for native sport had 13 observed variables (items) for 4 factors (ethnic identity: 3 items; acculturation: 4 items; IPSNC: 3 items; and CPSNC: 3 items) (see Figure 6).

Reliability and validity of the model. Factor loadings, Cronbach's alpha coefficients, and averaged extracted variance (AVE) for the final native sport measurement model were presented in Table 9. The Crongach's alpha coefficients for all the factors were greater than $.70(\alpha=.94$ for ethnic identity, $\alpha=.81$ for acculturation, $\alpha$ $=.94$ for IPSNC, and $\alpha=.88$ for CPSNC). 


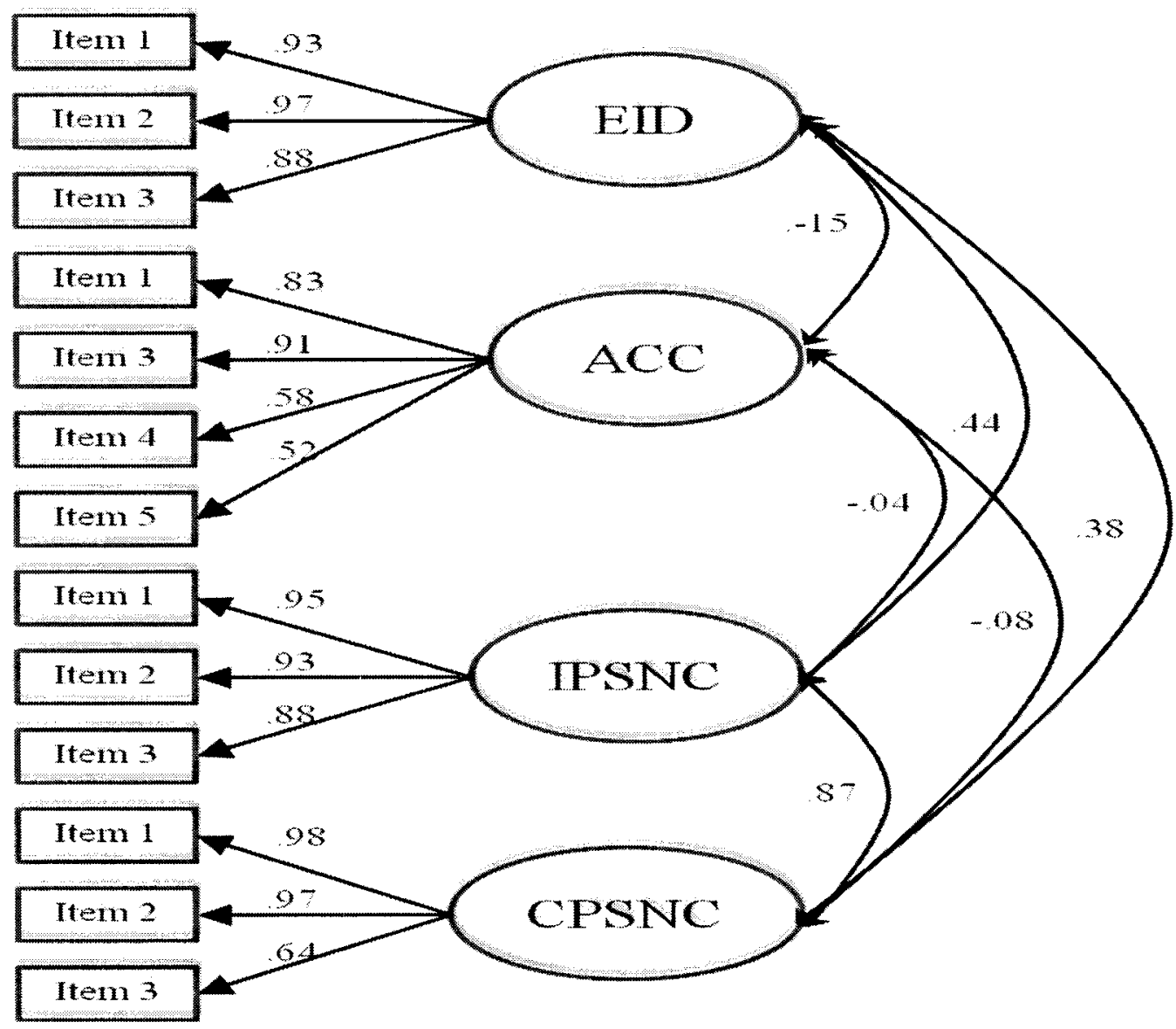

Figure 6. The Final Measurement Model for Native Sport. Note. EID = Ethnic Identity; ACC = Acculturation; IPSNC = Identification with a Popular Sport in a Native Country; and $\mathrm{CPSNC}=$ Consumption for a Popular Sport in a Native Country.

The AVE values for the four factors were all above the cutoff of .50 (Fornell \& Lacker, 1981) $(\mathrm{AVE}=.85$ for ethnic identity, $\mathrm{AVE}=.53$ for acculturation, $\mathrm{AVE}=.84$ for IPSNC, and AVE $=.74$ for CPSNC). With the exception of the correlation between IPSNC and CPSNC ( $r=.86)$, all correlations among the four factors were less than .85 (Kline, 2005) (see Table 10). The high correlation between IPSNC and CPSNC was expected since people in high levels of identification with a popular sport in a native country are more likely to consume the sport. The most AVE values were greater than the 
squared correlation between one factor and other factors. However, the squared correlation between IPSNC and CPSNC $\left(r^{2}=.75\right)$ was slightly above the AVE value of CPSNC (.74). Overall, the above results indicated that the measurement model for ethnic player showed appropriate internal consistency reliability and construct validity (e.g., discriminant validity).

Table 9.

Factor Loadings ( $\beta$ ), Cronbach's Alpha Coefficients, and AVE for the Final Native Sport Measurement Model

\begin{tabular}{llllc}
\hline Name of Factor & Item & $\beta$ & $\alpha$ & AVE \\
\hline Ethnic Identity & & & .94 & .85 \\
& Item 1 & .93 & & \\
& Item 2 & .97 & & \\
\hline Acculturation & Item 3 & .88 & & .53 \\
& & & .81 & \\
& Item 1 & .83 & & \\
& Item 3 & .92 & & \\
\hline IPSNC & Item 4 & .58 & & \\
& Item 5 & .52 & & \\
& & & .94 & \\
& Item 1 & .95 & & \\
\hline CPSNC & Item 2 & .93 & & \\
& Item 3 & .88 & & \\
& & & & \\
& Item 1 & .98 & & \\
\hline
\end{tabular}

Table 10.

Correlations among All the Four Factors for Native Sport Model

\begin{tabular}{lllll}
\hline Factor & 1 & 2 & 3 & 4 \\
\hline
\end{tabular}

1. Ethnic ID $\quad 1.00$


2. Acculturation $\quad-.151 \quad 1.00$

$\begin{array}{llll}\text { 3. IPSNC } & .444 & -.036 & 1.00\end{array}$

$\begin{array}{lllll}\text { 4. CPSNC } & .379 & -.079 & .868 & 1.00\end{array}$

\section{Host Sport Model}

This measurement model specified four latent factors, consisting of 17 items (ethnic identity: three items; acculturation: six items; identification with a popular sport in a host country (IPSHC): three items; and consumption for a popular sport in a host country (CPSHC): five items), to be correlated with each other. The results of the initial CFA indicated that this model showed unacceptable fit to the data based upon several fit indices $\left(x^{2} / d f=568.830 / 113=5.034\right.$, RMSEA $=.118, \mathrm{TLI}=.882, \mathrm{CFI}=.902, \mathrm{SRMR}$ $=.059)$. Similar to the previous two measurement models, theoretical relevance, empirical criteria (e.g., factor loadings, modification index (MI), standardized residuals), and parsimoniousness of the model were collectively considered in reaching a final decision regarding which items to retain and which to eliminate. Factor loadings for the initial CFA were presented in Table 11.

Table 11.

Factor Loadings ( $\beta$ ) of the 17 items for Host Sport Measurement Model

\begin{tabular}{lcc}
\hline Name of Factor & Item & Factor Loading \\
\hline \multirow{3}{*}{ Ethnic Identity } & Item 1 & .927 \\
& Item 2 & .975 \\
& Item 3 & .879 \\
\hline \multirow{3}{*}{ Acculturation } & Item 1 & .814 \\
& Item 2 & .461 \\
& Item 3 & .924
\end{tabular}




\begin{tabular}{lcc} 
& Item 4 & .593 \\
& Item 5 & .522 \\
& Item 6 & .075 \\
\hline \multirow{2}{*}{ IPSNC } & Item 1 & .949 \\
& Item 2 & .960 \\
\hline \multirow{2}{*}{ CPSNC } & Item 3 & .869 \\
\hline & Item 1 & .919 \\
& Item 2 & .949 \\
& Item 3 & .832 \\
\hline
\end{tabular}

After thoroughly checking factor loadings, item 6 of the acculturation factor (How negative do you feel about people of American culture?) was deleted because the item's factor loading ( $\beta=.075, p=.227$ ) was not statistically significant. Non-significance of this item might be due to its negative wording. In addition to item 6 on acculturation, item 2 (How much do you interact and associate with people from American culture?) on acculturation was deleted because of relatively low factor loading $(\beta=.458)$ that was lower than the recommended value of .50 (Hair et al., 1998 ). Further, item 2 on acculturation had two standardized residuals over the benchmark of 2.58 (Joreskog \& Sorbom, 1998). Thus, items 2 and 6 of acculturation factor were deleted in order to improve the model fit. These two items deletion were the same as the previous two measurement model.

After deleting the two items (items 2 and 6 of acculturation), the second CFA was done with the 15 items of the host sport measurement model. The results of the second CFA indicated that the revised measurement model did not still adequately fit to the data 
$\left(x^{2} / \mathrm{df}=463.368 / 84=5.323, \mathrm{RMSEA}=.125, \mathrm{TLI}=.895, \mathrm{CFI}=.916, \mathrm{SRMR}=.051\right) . \mathrm{As}$ a way of improving the model fit, modification indices (MIs) and standardized residuals were examined. In the second CFA, the highest MI (178.727) was a correlated error between items 4 and 5 of CPSHC. Additionally, item 5 had the highest standardized residual (3.622). Based upon the MI and standardized residual, item 5 of CPSHC factor (I buy the clothing of a popular sport in America) was deleted. Besides empirical reasons, this item deletion was also theoretically supported because item 5 had similar wording with item 4 within the same factor (I purchase the merchandise of a popular sport in America). The deletion of item 5 was based on the same theoretical and empirical reasons as those of the previous two measurement model.

Following the deletion of item 5, the third CFA was conducted with the remaining 14 items for the host sport measurement model. The third CFA results indicated that the measurement model showed a good fit to the data $\left(x^{2} / \mathrm{df}=181.699 / 71=2.559\right.$, RMSEA $=.073(90 \%$ confidence interval $=.06-08), \mathrm{TLI}=.965, \mathrm{CFI}=.972 \mathrm{SRMR}=.056)$.

Given the adequate fit of the model, no further modification was needed. The final measurement model for host sport had 14 observed variables (items) for 4 factors (ethnic identity: 3 items; acculturation: 4 items; IPSHC: 3 items; and CPSHC: 4 items) (see Figure 7). 


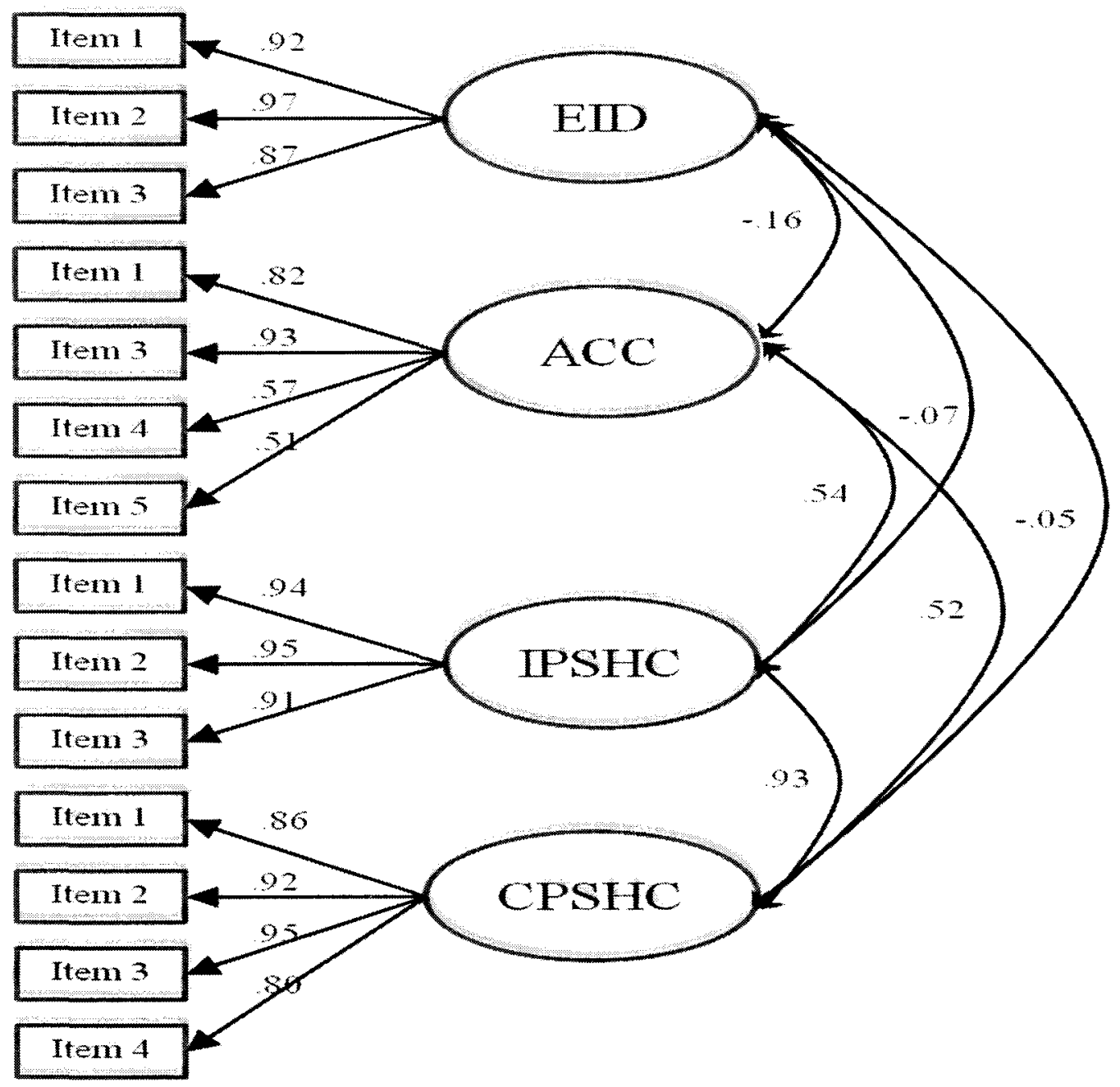

Figure 7. The Final Measurement Model for Host Sport. Note. EID = Ethnic Identity; $\mathrm{ACC}=$ Acculturation; IPSHC = Identification with a Popular Sport in a Host Country; and CPSNC $=$ Consumption for a Popular Sport in a Host Country.

Reliability and validity of the model. Factor loadings, Cronbach's alpha coefficients, and averaged extracted variance (AVE) for the final host sport measurement model were reported in Table 12. The Cronbach's alpha coefficients for all the factors were greater than $.70(\alpha=.94$ for ethnic identity, $\alpha=.81$ for acculturation, $\alpha=.95$ for IPSHC, and $\alpha=.93$ for CPSHC). All correlations among the four factors were less than .85 , with the exception of correlation between IPSHC and CPSHC ( $r=.92$ ) (Kline, 
2005) (see Table 10). The high correlation between IPSHC and CPSHC was also expected since people having high levels of identification with a popular sport in a host are more likely to consume the sport. The AVE values for three of the four factors were greater than the squared correlation between one factor and other factors. However, the squared correlation between IPSHC and CPSHC $\left(r^{2}=.84\right)$ was greater than the AVE value of CPSHC (.77). However, the overall above results indicated that the measurement model for ethnic player showed appropriate internal consistency reliability and construct validity (e.g., discriminant validity).

Table 12.

Factor Loadings ( $\beta$ ), Cronbach's Alpha Coefficients, and AVE for the Final Host Sport Measurement Model

\begin{tabular}{lllll}
\hline Name of Factor & Item & $\beta$ & $\alpha$ & AVE \\
\hline Ethnic Identity & & & .94 & .84 \\
& Item 1 & .92 & & \\
& Item 2 & .97 & & \\
\hline Acculturation & Item 3 & .87 & & .52 \\
& Item 1 & & .81 & .82 \\
& Item 3 & .93 & & \\
& Item 4 & .57 & & \\
IPSNC & Item 5 & .51 & & \\
& & & .95 & .86 \\
& Item 1 & .94 & & \\
& Item 2 & .95 & & \\
\hline CPSNC & Item 3 & .91 & & .77 \\
& & & .93 & \\
& Item 1 & .86 & & \\
& Item 2 & .92 & & \\
\hline
\end{tabular}

Table 13. 
Correlations among All the Four Factors for Host Sport Model

\begin{tabular}{lcccc}
\hline Factor & 1 & 2 & 3 & 4 \\
\hline 1. Ethnic ID & 1.00 & & & \\
2. Acculturation & -.157 & 1.00 & & \\
3. IPSHC & -.069 & .544 & 1.00 & \\
4. CPSHC & -.054 & .521 & .927 & 1.00 \\
\hline
\end{tabular}

\section{Structural Models}

Following the measurement model tests were intended to test structural models examining relationships among the constructs in each of the three proposed models. This data analysis procedure allowed the researcher to address Research Questions 1, 2, and 3. Below are detailed results of three structural models.

\section{Ethnic Player Structural Model (Research Question 1)}

The structural regression analysis tested the relationships among ethnic identity, acculturation, identification with an ethnic player (IEP), and consumption for a team with ethnic player (CTEP). The results of the analysis showed that the structural model had the identical model fit to the data with that of the final measurement model for ethnic player $\left(x^{2} / d f=189.768 / 71=2.673, \mathrm{RMSEA}=.076, \mathrm{TLI}=.960, \mathrm{CFI}=.969, \mathrm{SRMR}=.052\right)$.

The main reason that the structural model had the same fit with the measurement model was due to the fact that the numbers of free parameters to be estimated in the structural model were the same as those in the measurement model (34). The path coefficient of acculturation to IEP $(\beta=-.05, p=.368)$ and that of ethnic identity to CTEP $(\beta=.06, p$ $=.090)$ were not statistically significant. However, the path coefficient of ethnic identity to $\operatorname{IEP}(\beta=.42, p<.001)$ and that of acculturation to $\operatorname{CTEP}(\beta=.14, p<.001)$ were 
significant. Specifically, ethnic identity explained approximately $17.6 \%$ of variance in IEP and acculturation explained approximately $2.0 \%$ of variances in CTEP. In addition, the path coefficient of IEP to CTEP $(\beta=.89, p<.001)$ was significant. Specifically, IEP explained $79 \%$ of variance in CTEP. Lastly, the correlation between ethnic identity and acculturation was negatively significant $(r=-.15, p=.025)$. The structural model that examined the relationships among ethnic identity, acculturation, IEP, and CTEP is presented in Figure 8.

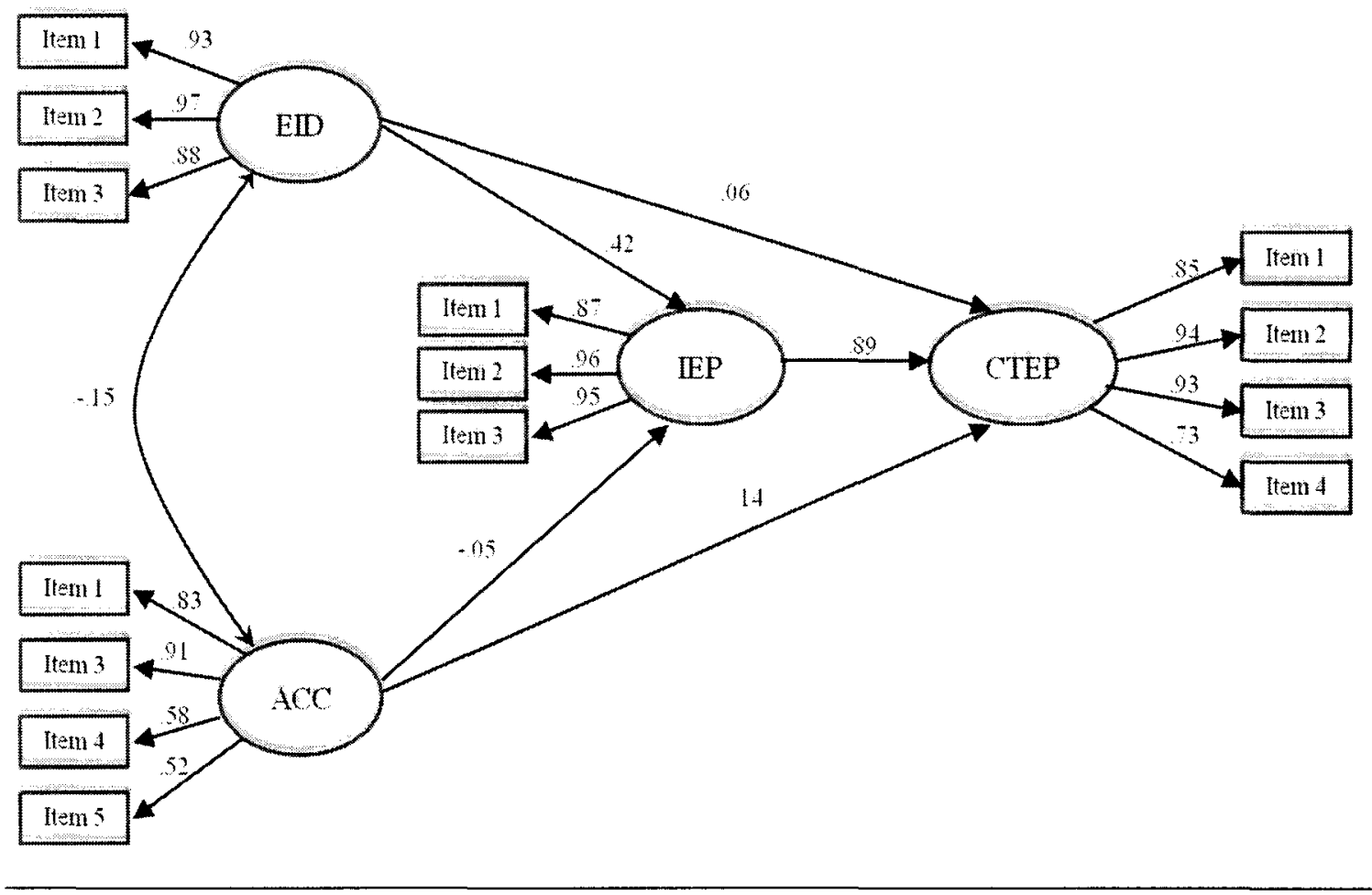

Figure 8. The Structural Model for Ethnic Player. Note. The significant relationships to be found were as follows: (a) the correlation between EID and ACC, (b) the relationship between EID and IEP, (c) the relationship between ACC and CTEP, and (d) the relationship between IEP and CTEP.

Native Sport Structural Model (Research Question 2) 
The structural regression analysis for this model tested the relationships among ethnic identity, acculturation, identification with a popular sport in a native country (IPSNC), and consumption for a popular sport in a native country (CPSNC). Like the structural model for ethnic player, the results of this analysis indicated that the structural model had the identical model fit to the data with that of the final measurement model for native sport $\left(x^{2} / \mathrm{df}=151.063 / 59=2.560\right.$, RMSEA $=.073, \mathrm{TLI}=.965, \mathrm{CFI}=.973$, SRMR $=.058)$. The main reason that the structural model had the same fit with the measurement model was due to the fact that the numbers of free parameters to be estimated in the structural model were the same as those in the measurement model (32). The path coefficients of acculturation to IPSNC $(\beta=.031, p=.592)$ and to CPSNC $(\beta=-.05, p$ $=.161$ ) were not significant. Further, the path coefficient of ethnic identity to CPSNC was not significant $(\beta=-.02, p=.671)$. However, the path coefficient of ethnic identity to IPSNC $(\beta=.45, p<.001)$ and that of IPSNC to CPSNC $(\beta=.87, p<.001)$. More specifically, ethnic identity explained approximately $20.2 \%$ of variance in IPSNC and IPSNC explained approximately $75.6 \%$ of variance in CPSNC. Lastly, the correlation between ethnic identity and acculturation was negatively significant $(r=-.15, p=.019)$. The structural model that examined the relationships among ethnic identity, acculturation, IPSNC, and CPSNC is presented in Figure 9. 


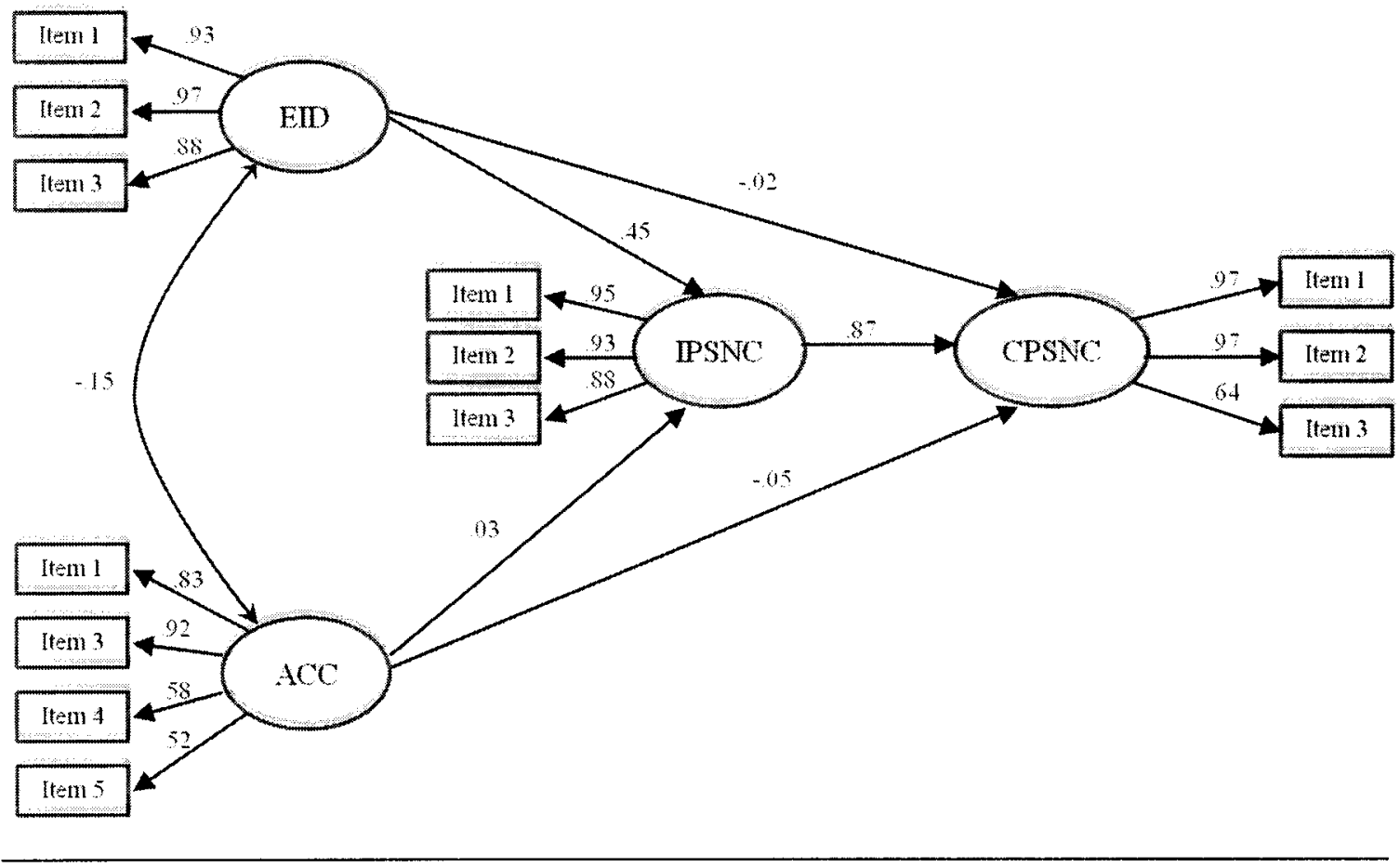

Figure 9. The Structural Model for Native Sport Model. Note. The significant relationships to be found were as follows: (a) the correlation between EID and ACC, (b) the relationship between EID and IPSNC, and (c) the relationship between IPSNC and CPSNC.

\section{Host Sport Structural Model (Research Question 3)}

The structural regression analysis for this model tested the relationships among ethnic identity, acculturation, identification with a popular sport in a host country (IPSHC), and consumption for a popular sport in a native country (CPSHC). Like the previous two structural models, this structural model had also the same model fit to the data with that of the final measurement model for host sport $\left(x^{2} / \mathrm{df}=181.699 / 71=2.559\right.$, RMSEA $=.073(90 \%$ confidence interval $=.06-08), \mathrm{TLI}=.965, \mathrm{CFI}=.972 \mathrm{SRMR}$ $=.056)$. Likewise, the main reason that the structural model had the same fit with the measurement model was due to the fact that the numbers of free parameters to be 
estimated in the structural model were the same as those in the measurement model (34). The path coefficients of ethnic identity to IPSHC ( $\beta=.02, p=.742)$ and to CPSHC ( $\beta$ $=.01, p=.645$ ) were not significant. Further, the path coefficient of acculturation to CPSHC was not significant $(\beta=.03, p=.476)$. However, the path coefficient of acculturation to IPSHC $(\beta=.55, p<.001)$ and that of IPSHC to CPSHC $(\beta=.91, p<$ .001 ) was significant. More specifically, acculturation explained approximately $30.2 \%$ of variance in IPSHC and IPSHC explained approximately $82.8 \%$ of variance in CPSHC. Lastly, the correlation between ethnic identity and acculturation was negatively significant $(r=-.16, p=.014)$. The structural model that examined the relationships among ethnic identity, acculturation, IPSHC, and CPSHC is presented in Figure 10.

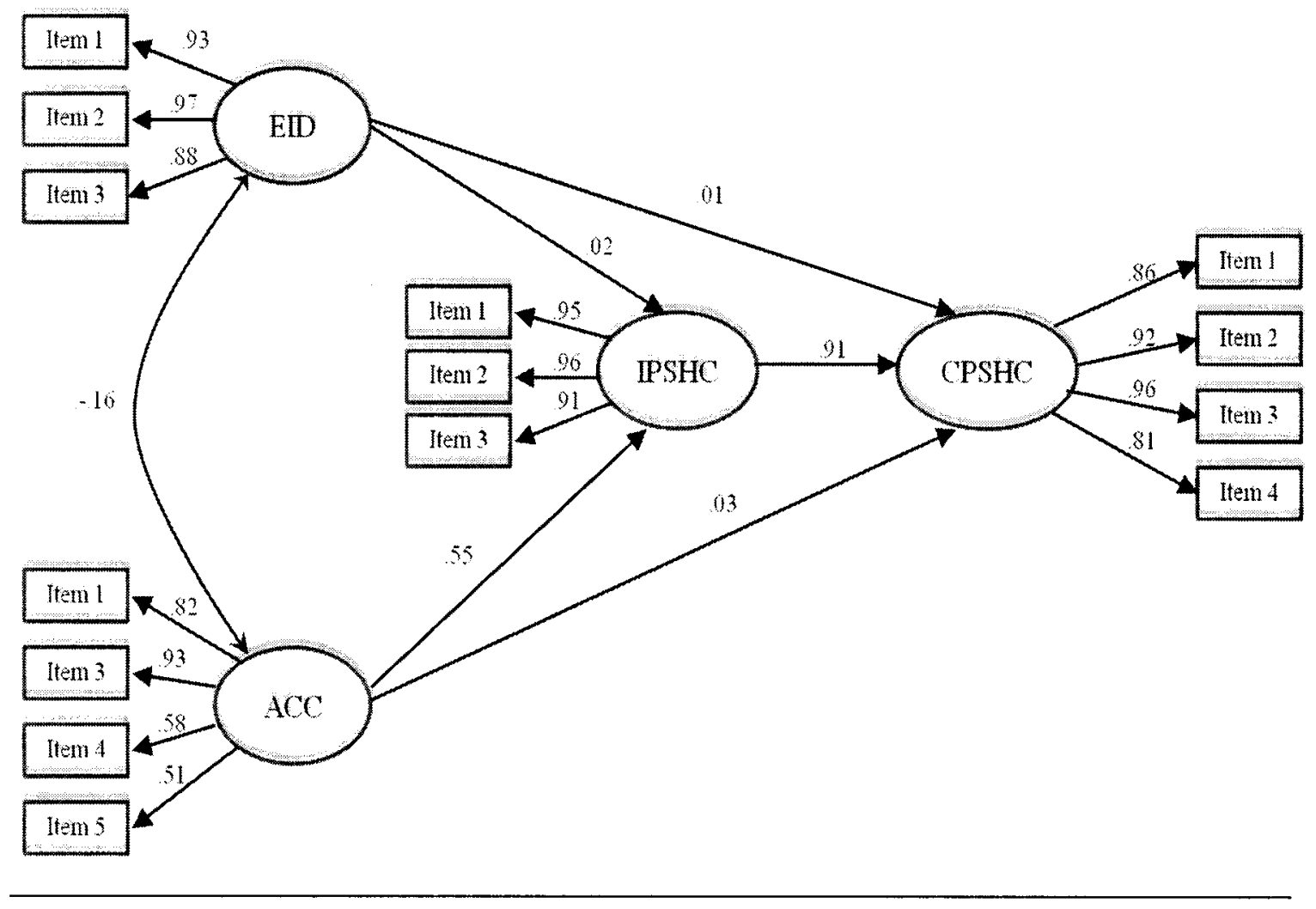

Figure 10. The Structural Model for Host Sport Model. Note. The significant relationships to be found were as follows: (a) the correlation between EID and ACC, (b) 
the relationship between ACC and IPSHC, and (d) the relationship between IPSHC and CPSHC.

\section{Results of MANCOVA}

Three separate MANCOVAs were carried out to address Research Questions 4-1, 4-2, and 4-3.The three sub-Research Questions specifically examined the impact of four acculturation strategies (integration, assimilation, separation, and marginalization) on three types of identifications and three types of sport consumptions, while controlling for the effect of length of residence in the U.S. According to prior research, the longer immigrants live in a host country, the more likely their consumption level of products (e.g., food, sport) of the host country would increase (Boeckner, Jordan, \& Schnepf, 2000; Ha, Hums, \& Greenwell, 2011). Therefore, it is important to control for the length of residence in the U.S. within the analysis.

Using a median split, the four acculturation strategies were classified. The median scores for native culture (AAMAS - CO) and host culture (AAMAS - EA) dimensions were 4.75 and 4.25 , respectively. Respondents who scored at or above the median on both dimensions were sorted into integration strategy, whereas those who scored below the median on both dimensions were grouped in the marginalization strategy. In the same manner, participants who scored at or above the median on native culture but below the median on host culture were classified in the separation strategy, whereas those who scored below the median on native culture but at or above the median on host culture were grouped in the assimilation strategy. Base on the median split, there were $70(24.1 \%)$ for integration strategy, $85(29.2 \%)$ for assimilation, $76(26.1 \%)$ for separation, and 60 $(20.6 \%)$ for marginalization. 


\section{Assumptions for MANCOVA}

Prior to conducting MANCOVAs, several assumptions need to be met (Stevens, 2005). These assumptions included normal distribution of dependent variables, equality of variance-covariance matrices, a linear relationship between dependent variables and the covariate (controlling variable), and homogeneity of regression slopes, meaning that the slopes of the regression lines for each level of an independent variable are equal. All of the above assumption checks occurred prior to conducting MANCOVAs.

The skewness and kurtosis values for dependent variables in all the three MANCOVAs were within an acceptable range of \pm 2 (George \& Malley, 2001), suggesting that the normality assumption was met: (a) IEP (skewness $=.194$ and kurtosis $=-.993$ ) and CTEP (skewness $=.693$ and kurtosis $=-.332) ;(\mathrm{b})$ IPSNC (skewness $=.616$ and kurtosis $=-.558)$ and CPSNC (skewness $=1.220$ and kurtosis $=.946)$; and (c) IPSHC $($ skewness $=.836$ and kurtosis $=-.238)$ and CPSHC $($ skewness $=1.166$ and .716$)$. Box's $\mathrm{M}$ tests were significant in all the three MANCOVAs, indicating that the equality of covariance matrices assumption was not met: (a) the first MANCOVA for IEP and CTEP $[F(9,686699.564)=4.21, p<.01)] ;(\mathrm{b})$ the second MANCOVA for IPSNC and CPSNC $[F(9,686699.564)=6.06, p<.01)]$; and $(c)$ the third MANCOVA for IPSHC and CPSHC $[F(9,686699.564)=9.69, p<.01)]$. However, Field $(2009)$ and Stevens $(2005)$ suggested that the $F$ statistic in multivariate analyses of variance is robust to violation of the homogeneity of covariance matrices assumption, so long as group sample sizes are relatively equal (Field, 2009; Stevens, 2005). In addition, if the largest group size is less than 1.5 times of the smallest group size, it is robust to violation of this assumption 
(Stevens). In the present study, all group sizes for acculturation strategies were within this range.

Another critical assumption to be met is a linear relationship between dependent variables and the covariate. Within the first MANCOVA, the multivariate relationship between the dependent variables (IEP and CTEP) and the covariate (length of residence in the U.S.) was not significant, Wilks' $\Lambda=.993, F(2,273)=1.01, p>.05$. However, the multivariate relationships between the dependent variables (IPSNC and CPSNC) and the covariate in the second MANCOVA [Wilks' $\Lambda=3.128, F(2,273)=3.138, p<.05$.] and between the dependent variables (IPSHC and CPSHC) and the covariate in the third MANCOVA [Wilks' $\Lambda=3.128, F(2,273)=3.332, p<.05$.] were significant. Despite the non-significant result of the first MANCOVA, the researcher utilized the length of residence in the U.S. as a covariate within the analysis based on prior research (Ha et al., in review).

The last assumption to be checked was the homogeneity of regression slopes assumption. This assumption was met in all the three MANCOVAs, indicating that the covariate-by-treatment (acculturation strategies) interaction between groups was not significant (Stevens, 2005): (a) the first MANCOVA for IEP and CTEP [Wilks' $\Lambda=.985$, $F(6,540)=.671, p>.05)] ;(\mathrm{b})$ the second MANCOVA for IPSNC and CPSNC [Wilks' $\Lambda$ $=.958, F(6,540)=.453, p>.05)] ;$ and $(\mathrm{c})$ the third MANCOVA for IPSHC and CPSNC $\left[\right.$ Wilks' $\left.\left.^{\prime} \Lambda=.981, F(6,540)=.864, p>.05\right)\right]$

\section{Table 14.}

Summaries of Assumption Checks for MANCOVAs

Assumptions

\begin{tabular}{llll}
\hline Types of Normality Equality of Linear Relationship Homogeneity &
\end{tabular}




\begin{tabular}{lcccc}
\hline MANCOVAs & & $\begin{array}{l}\text { Covariance } \\
\text { Matrices }\end{array}$ & $\begin{array}{l}\text { between the DVs and } \\
\text { the Covariate }\end{array}$ & $\begin{array}{l}\text { of Regression } \\
\text { Slopes }\end{array}$ \\
\hline $\begin{array}{l}\text { The First } \\
\text { MANCOVA }\end{array}$ & Met & Not Met & Not Met & Met \\
$\begin{array}{l}\text { The Second } \\
\text { MANCOVA }\end{array}$ & Met & Not Met & Met & Met \\
$\begin{array}{l}\text { The Third } \\
\text { MANCOVA }\end{array}$ & Met & Not Met & Met & Met \\
\hline
\end{tabular}

Note. (a) Dependent variables (DVs) in the first MANCOVA were IEP and CTEP; (b)

DVs in the second MANCOVA were IPSNC and CPSNC; and (c) DVs in the third MANCOVA were IPSHC and CPSHC. Covariate in the three MANCOVAS was the length of residence in the U.S.

\section{Research Question 4-1 (The Impact of Acculturation Strategies on IEP and CTEP)}

The results of the first MANCOVA revealed a significant multivariate effect of acculturation strategies on IEP and CTEP [Wilks' $\Lambda=.790, F(6,546)=11.39, p<.001$ ]. The follow-up univariate analyses were conducted to inspect the nature of differences. The analyses revealed a significant effect of acculturation strategies on $\operatorname{IEP}[F(3,274)=$ $\left.21.29, p<.001, \eta^{2}=.189\right]$ and CTEP $\left[F(3,274)=20.92, p<.001, \eta^{2}=.186\right)$.

Pairwise comparisons were performed to examine differences in IEP and CTEP among four acculturation strategies. For IEP, significant differences were found between integration $(M=4.47)$ and assimilation $(M=2.82)$, between integration and marginalization $(M=3.05)$, between assimilation and separation $(M=4.37)$, and between separation and marginalization. However, no significant differences were found between integration and separation, and between assimilation and marginalization. Table 15 showed pairwise comparisons among four acculturation strategies with respect to identification with an ethnic player (IEP). 
Table 15.

Pairwise Comparisons of Identification with Ethnic Playrer (IEP) by Acculturation

Strategies.

\begin{tabular}{|c|c|c|c|c|c|c|}
\hline & & & \multicolumn{4}{|c|}{ Adjusted Mean Differences } \\
\hline $\begin{array}{l}\text { Acculturation } \\
\text { Strategies }\end{array}$ & Mean & $\begin{array}{l}\text { Adjusted } \\
\text { Mean }\end{array}$ & 1. & 2. & 3. & 4. \\
\hline $\begin{array}{l}\text { 1. Integration } \\
\text { Strategy }(n=70)\end{array}$ & 4.42 & 4.47 & - & & & \\
\hline $\begin{array}{l}\text { 2. Assimilation } \\
\text { strategy }(n=85)\end{array}$ & 2.65 & 2.82 & $1.65 * * *$ & - & & \\
\hline $\begin{array}{l}\text { 3. Separation } \\
\text { Strategy }(n=76)\end{array}$ & 4.42 & 4.37 & .10 & $1.55 * * *$ & - & \\
\hline $\begin{array}{l}\text { 4. Marginalization } \\
\text { Strategy }(n=60)\end{array}$ & 3.14 & 3.05 & $1.42 * * *$ & .23 & $1.32 * * *$ & - \\
\hline
\end{tabular}

Likewise, pairwise comparisons for CTEP indicated a significant difference between the same paring groups as those in the IEP. In other words, there was a significant difference between integration $(M=3.46)$ and assimilation $(M=2.08)$, between integration and marginalization $(M=2.18)$, between assimilation and separation $(M=3.18)$, and between separation and marginalization. However, there were no significant differences between integration and separation, and between assimilation and marginalization. Table 16 presented pairwise comparisons among four acculturation strategies with respect to consumption for a team with an ethnic player (CTEP).

Table 16.

Pairwise Comparisons of Consumption for a Team with Ethnic Player (CTEP) by Acculturation Strategies 
Adjusted Mean Differences

\begin{tabular}{lccllll}
\hline $\begin{array}{l}\text { Acculturation } \\
\text { Strategies }\end{array}$ & Mean & $\begin{array}{c}\text { Adjusted } \\
\text { Mean }\end{array}$ & 1. & 2. & 3. & 4. \\
$\begin{array}{l}\text { 1. Integration } \\
\text { Strategy }(\mathrm{n}=70)\end{array}$ & 3.43 & 3.46 & - & & & \\
$\begin{array}{l}\text { 2. Assimilation } \\
\text { strategy (n=85) }\end{array}$ & 2.03 & 2.08 & $1.40^{* * *}$ & - & & \\
$\begin{array}{l}\text { 3. Separation } \\
\text { Strategy (n=76) }\end{array}$ & 3.21 & 3.18 & .28 & $1.10^{* * *}$ & - & \\
$\begin{array}{l}\text { 4. Marginalization } \\
\text { Strategy }(\mathrm{n}=60)\end{array}$ & 2.23 & 2.18 & $1.28^{* * *}$ & .10 & $1.00^{* * *}$ & - \\
\hline$* p<.05, * * p<.01, * * * p<.001$ & & & & &
\end{tabular}

Research Question 4-2 (The Impact of Acculturation Strategies on IPSNC and CPSNC)

The results of the second MANCOVA revealed a significant multivariate effect of acculturation strategies on IPSNC and CPSNC [Wilks' $\Lambda=.767, F(6,546)=11.39, p$ $<.01]$. The follow-up univariate analyses were conducted to inspect the nature of differences. The analyses revealed a significant effect of acculturation strategies on $\operatorname{IPSNC}\left[F(3,274)=27.11, p<.01, \eta^{2}=.229\right]$ and $\operatorname{CPSNC}\left[F(3,274)=19.15, p<.01, \eta^{2}\right.$ $=.173)]$.

Pairwise comparisons were performed to examine differences in IPSNC and CPSNC among four acculturation strategies. For IPSNC, significant differences were found between integration $(M=3.83)$ and assimilation $(M=2.12)$, between integration and marginalization $(M=2.26)$, between assimilation and separation $(M=3.67)$, and between separation and marginalization. However, no significant differences were found between integration and separation, and between assimilation and marginalization. Table 
17 showed pairwise comparisons among four acculturation strategies with respect to identification with a popular sport in a native country (IPSNC).

Table 17.

Pairwise Comparisons of Identification with a Popular Sport in a Native Country (IPSNC) by Acculturation Strategies.

Adjusted Mean Differences

\begin{tabular}{|c|c|c|c|c|c|c|}
\hline $\begin{array}{l}\text { Acculturation } \\
\text { Strategies }\end{array}$ & Mean & $\begin{array}{l}\text { Adjusted } \\
\text { Mean }\end{array}$ & 1. & 2. & 3. & 4. \\
\hline $\begin{array}{l}\text { 1. Integration } \\
\text { Strategy }(n=70)\end{array}$ & 3.76 & 3.83 & - & & & \\
\hline $\begin{array}{l}\text { 2. Assimilation } \\
\text { strategy }(n=85)\end{array}$ & 2.00 & 2.12 & $1.71 * * *$ & - & & \\
\hline $\begin{array}{l}\text { 3. Separation } \\
\text { Strategy }(n=76)\end{array}$ & 3.75 & 3.67 & .16 & $1.55 * * *$ & - & \\
\hline $\begin{array}{l}\text { 4. Marginalization } \\
\text { Strategy }(n=60)\end{array}$ & 2.40 & 2.26 & $1.57 * * *$ & .14 & $1.41 * * *$ & - \\
\hline
\end{tabular}

In the same vein, pairwise comparisons for CPSNC indicated a significant difference between the same paring groups as those in the IPSNC. Specifically, there was a significant difference between integration $(M=2.68)$ and assimilation $(M=1.51)$, between integration and marginalization $(M=1.72)$, between assimilation and separation $(M=2.63)$, and between separation and marginalization. However, no significant differences were found between integration and separation, and between assimilation and marginalization. Table 18 presented pairwise comparisons among four acculturation strategies with respect to consumption for a popular sport in a native country (CPSNC). Table 18. 
Pairwise Comparisons of Consumption for a Popular Sport in a Native Country (CPSNC) by Acculturation Strategies

Adjusted Mean Differences

\begin{tabular}{lccllll}
\hline $\begin{array}{l}\text { Acculturation } \\
\text { Strategies }\end{array}$ & Mean & $\begin{array}{c}\text { Adjusted } \\
\text { Mean }\end{array}$ & 1. & 2. & 3. & 4. \\
$\begin{array}{l}\text { 1. Integration } \\
\text { Strategy }(\mathrm{n}=70)\end{array}$ & 2.63 & 2.68 & - & & & \\
$\begin{array}{l}\text { 2. Assimilation } \\
\text { strategy (n=85) }\end{array}$ & 1.43 & 1.51 & $1.17^{* * *}$ & - & & \\
$\begin{array}{l}\text { 3. Separation } \\
\text { Strategy (n=76) }\end{array}$ & 2.68 & 2.63 & .05 & $1.12^{* * *}$ & - & \\
$\begin{array}{l}\text { 4. Marginalization } \\
\text { Strategy }(\mathrm{n}=60)\end{array}$ & 1.82 & 1.72 & $.96^{* * *}$ & .21 & $.91 * * *$ & - \\
\hline$* p<.05, * * p<.01, * * * p<.001$ & & & & & &
\end{tabular}

Research Question 4-3 (The Impact of Acculturation Strategies on IPSHC and CPSHC

The results of the third MANCOVA revealed a significant multivariate effect of acculturation strategies on IPSHC and CPSHC [Wilks' $\Lambda=.782, F(6,546)=11.92, p$ $<.01]$. The follow-up univariate analyses were conducted to inspect the nature of differences. The analyses revealed a significant effect of acculturation strategies on $\operatorname{IPSHC}\left[F(3,274)=24.92, p<.01, \eta^{2}=.214\right]$ and $\operatorname{CPSHC}\left[F(3,274)=17.08, p<.01, \eta^{2}\right.$ $=.158)]$.

Pairwise comparisons were conducted to investigate differences in IPSHC and CPSHC among four acculturation strategies. For IPSHC, significant differences were found between integration $(M=3.49)$ and separation $(M=1.98)$, between integration and marginalization $(M=1.77)$, between assimilation $(M=3.38)$ and separation, and between 
assimilation and marginalization. No significant differences, however, were detected between integration and assimilation, or between separation and marginalization. Table 19 shows pairwise comparisons among four acculturation strategies with regard to identification with a popular sport in a host country (IPSHC).

Table 19.

Pairwise Comparisons of Identification with a Popular Sport in a Host Country (IPSHC) by Acculturation Strategies.

Adjusted Mean Differences

\begin{tabular}{|c|c|c|c|c|c|c|}
\hline $\begin{array}{l}\text { Acculturation } \\
\text { Strategies }\end{array}$ & Mean & $\begin{array}{l}\text { Adjusted } \\
\text { Mean }\end{array}$ & 1. & 2. & 3. & 4. \\
\hline $\begin{array}{l}\text { 1. Integration } \\
\text { Strategy }(n=70)\end{array}$ & 3.54 & 3.49 & - & & & \\
\hline $\begin{array}{l}\text { 2. Assimilation } \\
\text { strategy }(\mathrm{n}=85)\end{array}$ & 3.45 & 3.38 & .11 & - & & \\
\hline $\begin{array}{l}\text { 3. Separation } \\
\text { Strategy }(n=76)\end{array}$ & 1.93 & 1.98 & $1.51^{* * *}$ & $1.40^{* * *}$ & - & \\
\hline $\begin{array}{l}\text { 4. Marginalization } \\
\text { Strategy }(n=60)\end{array}$ & 1.68 & 1.77 & $1.72 * * *$ & $1.61^{* * *}$ & .21 & - \\
\hline
\end{tabular}

Likewise, pairwise comparisons for CPSHC indicated a significant difference between the same paring groups as those in the IPSHC. Specifically, there was a significant difference between integration $(M=3.10)$ and separation $(M=1.88)$, between integration and marginalization $(M=1.83)$, between assimilation $(M=2.93)$ and separation, and between assimilation and marginalization. However, no significant differences were found between integration and assimilation, and between separation and 
marginalization. Table 20 reported pairwise comparisons among four acculturation strategies with respect to consumption for a popular sport in a host country (CPSHC). Table 20.

Pairwise Comparisons of Consumption for a Popular Sport in a Host Country (CPSHC) by Acculturation Strategies.

\begin{tabular}{|c|c|c|c|c|c|c|}
\hline & & & \multicolumn{4}{|c|}{ Adjusted Mean Differences } \\
\hline $\begin{array}{l}\text { Acculturation } \\
\text { Strategies }\end{array}$ & Mean & $\begin{array}{l}\text { Adjusted } \\
\text { Mean }\end{array}$ & 1. & 2. & 3. & 4. \\
\hline $\begin{array}{l}\text { 1. Integration } \\
\text { Strategy }(n=70)\end{array}$ & 3.17 & 3.11 & - & & & \\
\hline $\begin{array}{l}\text { 2. Assimilation } \\
\text { strategy }(n=85)\end{array}$ & 3.02 & 2.93 & .18 & - & & \\
\hline $\begin{array}{l}\text { 3. Separation } \\
\text { Strategy }(n=76)\end{array}$ & 1.87 & 1.89 & $1.22 * * *$ & $1.04 * * *$ & - & \\
\hline $\begin{array}{l}\text { 4. Marginalization } \\
\text { Strategy }(n=60)\end{array}$ & 1.71 & 1.83 & $1.28 * * *$ & $1.10 * * *$ & .06 & - \\
\hline
\end{tabular}

\section{Summary of Results}

The current study tested three Sport Consumption Models (see Figure 1-1, 1-2, and 1-3 in Chapter I) for the Asian population living in the U.S. based on ethnic identity and acculturation. The first model (Ethnic Player Model) involved acculturation, ethnic identity, identification with an ethnic player (IED), and consumption for a team with ethnic player (CTEP), the second model (Native Sport Model) included the two cultural factors, identification with a popular sport in a native country (IPSNC), and consumption for a popular sport in a native country (CPSNC), and the third model (Host Sport Model) included the two cultural factors, identification with a popular sport in a host country 
(IPSHC), and consumption for a popular sport in a host country (CPSHC). Based on theoretical and empirical criteria (e.g., factor loading, standardized residual, modification index), and parsimoniousness of the models, two items (items 2 and 6) on the acculturation factor and one item on the consumption factor were deleted in all the three measurement models using CFAs (item 5 of Ethnic Player Model and Host Sport Model and item 4 of Native Sport Model). The overall reliability and construct validity of the three measurement models were established by the AVE value and Cronbach's alpha. After deleting the items, the three structural models were tested. All the three structural models did adequately fit to the data based on several fit indices (e.g., $x^{2} / \mathrm{df}$, RMSEA, TLI, CFI, SRMR).

Within all the three structural models, ethnic identity and acculturation were negatively correlated. Ethnic identity significantly influenced identification in the Ethnic Player (IEP) and Native Sport (IPSNC) Models. However, ethnic identity did not directly influence consumption in all the three structural models. Acculturation significantly influenced only identification in the Host Sport Model (IPSHC). Regarding the relationship with consumption, acculturation only significantly influenced consumption in the Ethnic Player Model (CTEP). Lastly, within all the three structural models, identification significantly influenced consumption.

The study employed MANCOVAs to examine the impact of four acculturation strategies (integration, assimilation, separation, and marginalization) on three types of identifications (IEP, IPSNC, IPSHC) and three types of sport consumption (CTEP, CPSHC, CPSHC), while controlling for the effect of length of residence in the U.S. The 
results of the MANCOVAs revealed significant differences in the three identification and consumption factors among the four acculturation strategies. 


\section{CHAPTER V}

\section{DISCUSSION}

While a plethora of studies have investigated the effects of various socio-

demographic (e.g., gender, income, ethnicity) and psychological factors (e.g., motivation, identification, involvement, loyalty) on sport consumption behaviors, there were limited attempts to understand psycho-cultural factors, such as ethnic identity and acculturation, in the sport consumer literature (Ha, Ha, \& Greenwell, 2011;Harrole \& Trail, 2007; Pons et al., 2001). Two cultural factors (ethnic identity and acculturation) might be particularly critical for sport marketers to target ethnic minority populations as a niche market. Thus, the researcher identified the need to investigate the role of the two cultural factors in attracting various ethnic minorities to a sport consumption setting. Given the dramatic growth of the Asian population in the U.S. and its strong economic power, the current study focused solely on the following four Asian specific subgroups living in the U.S.: (a) Chinese, (b) Japanese, (c) Korean, and (d) Taiwanese.

The primary purpose of the current study was to develop sport consumption models for the Asian population living in the U.S., grounded in the two cultural constructs of ethnic identity and acculturation, and also identifications (points of attachment) and consumption associated with the respective identification. This study also aimed to examine the impact of the four acculturation strategies (integration, assimilation, separation, and marginalization) on identifications and sport consumption behaviors. This chapter first discusses theoretical implications based on the key finding 
related to descriptive statistics and Research Questions. Then, future research will be discussed. Lastly, a summary of the entire study will be presented.

\section{Major Implications of the Study}

The current study revealed several key findings regarding the mean scores of key variables, the roles of the two cultural factors (ethnic identity and acculturation) with three identification and three consumption variables for the respective identification, and the impact of acculturation strategies on the three identification and consumption variables. Based on these key findings, the current study had both theoretical and practical implications. In this section, theoretical implications are first discussed, followed by practical implications.

\section{Theoretical Implications}

Descriptive statistics for the key variables. The key research variables involved in the three proposed models for the current study were (a) ethnic identity, (b) acculturation, (c) three identification variables: [i] identification with an ethnic player (IEP), [ii] identification with a popular sport in a native country (IPSNC),[iii] identification with a popular sport in a host country (IPSHC); and (d) three sport consumption variables: [i] consumption for a team with an ethnic player (CTEP), [ii] consumption for a popular sport in a native country (CPSNC), [iii] consumption for a popular sport in a host country (CPSHC).

For the two cultural variables, the ethnic identity mean score (5.05) indicated respondents were highly identified with their countries of origin, while the acculturation mean score (4.32) revealed respondents were moderately acculturated to American culture. Regarding the three identification variables, the mean scores on IEP (3.65) and 
IPSNC (2.96) were higher than that of IPSHC (2.71). The findings are partially consistent with a previous study that found Asians were inclined to root for their ethnic players as a way of identifying with their ethnic group (Min \& Kim, 2009). The findings may be also due to the higher mean score of ethnic identity than that of acculturation in the current study. According to the literature, people having high levels of ethnic identity are likely to identify with and consume products/services reflecting attributes of their countries of origin (Cleveland, Laroche, Pons, \& Kasto 2009; Xu, Shim, Lotz, \& Almeida , 2004). For the sport consumption variables, the mean score on CTEP (2.71) was the highest, but still low, among the three consumption variables. As opposed to the identification variables, however, participants were less likely to consume native sports (CPSNC) than host sports (CPSHC). One possible explanation for these results is that participants might be less exposed to cultural-related sport products (e.g., a popular sport in a native country) since they currently live in the U.S. (host country).

\section{Research Question 1 (ethnic player structural model). Research Question 1} pertaining to the Ethnic Player Structural Model addressed the relationships among ethnic identity, acculturation, identification with an ethnic player (IEP), and consumption for a team with ethnic player (CTEP). First, the results indicated ethnic identity had a very low and negative correlation with acculturation. This finding is consistent with Berry's bidimensional model of acculturation $(1990,1997)$ suggesting that acculturation and ethnic identity are two independent but related constructs. In other words, people high in ethnic identity did not necessarily report low levels of acculturation and vice versa. Jun, Ball, and Gentry's (1993) work also revealed a similar result by finding cultural identity and acculturation to be distinctive constructs. Second, ethnic identity significantly and 
positively influenced identification with ethnic player (IEP) as expected. This finding is supported by prior literature indicating that Koreans living in the U.S. tend to cheer for players from their countries of origin in order to maintain ethnic identity (Min \& Kim, 2009). Additionally, this finding may be consistent with a recent study that noted an individual's patriotism (similar to ethnic identity) (Rocha, 2011) would increase by identifying with his/her national athletes playing in the U.S. Further, Kim and Cheong (2011) found that consumers are inclined to show more favorable attitudes toward a sponsor's brand in the context of athlete endorsement when the ethnicity of the athlete matched that of the consumers.

Third, ethnic identity did not directly influence consumption for a team with ethnic player (CTEP). This result was different than expected. One plausible explanation of this result is that respondents in the current study showed relatively little interest in the professional teams on which their ethnic players played $(M=2.71)$. That is, even if Asians are identified with their specific ethnic players, they do not take the teams' athletic performance seriously into account. Rather, they primarily focus on individual ethnic players' athletic performance in order to maintain identification with their specific ethnic group. However, given that ethnic identity indirectly influenced CTEP through IEP, it is plausible that Asians maintaining identity with their countries of origin are likely to consume a team's games in which their ethnic player are playing by identifying with the ethnic player.

Fourth, acculturation did marginally and positively influence CTEP, but did not affect IEP. This finding was slightly different than expected based on the current literature. One possible reason acculturation marginally influenced CTEP is that although 
the current study did not directly examine the levels of team identification, respondents, in spite of being newcomers (e.g., immigrants, international students), may identify with their local professional teams and ultimately watch or attend the team's game in an attempt to acculturate into a local society where they reside (e.g., Seattle, Houston, Cleveland, and L.A. in this study). In addition, according to the literature, people utilize sport teams to identify with a community (community identity) (Heere \& James, 2007; Trail et al., 2003) even if they are newcomers. From this perspective, the above finding can be explained. However, Asians in the current study did not identify with their ethnic players as a means of adaptation to the host society (the U.S.). Rather, they utilized them to maintain ethnic identity.

The last finding pertaining to Research Question 1 was that IED significantly and positively influenced CTED. This finding is directly supported by many previous studies suggesting that fan identification is a significant predictor of sport consumption behavior (e.g., Fisher \& Wakefield, 1998; James \& Trail, 2008; Trail, Anderson et al., 2000; Wann \& Branscombe, 1993). In other words, highly identified fans consume greater quantities of sport (i.e., merchandise consumption, game attendance, media consumption) than their lesser identified counterparts. What this means is that Asians highly identified with their culture of origin players are more likely to attend and watch the team's games with an ethnic player, and even purchase merchandise associated with the team. For example, when Chan-Ho Park, the first Korean player to play in MLB, joined L.A. Dodgers as a pitcher in 1994, the Dodgers saw an rapid upturn in Korean attendance.

In summary, acculturation and ethnic identity were marginally and negatively associated with one another, indicating that people with low levels of ethnic identity do 
not necessarily show high levels of acculturation. Ethnic identity positively influenced IEP, but did not directly influence CTEP. With regard to the relationship between IED and CTEP, IED positively influenced CTEP. These findings imply that ethnic identity indirectly affects CTEP. What this means is, for example, that a Japanese person highly identified with his/her country of origin is likely to watch Seattle Mariners' games by identifying with Ichiro Suzuki. Acculturation marginally and positively influenced CTEP, but did not influence IEP.

Research Question 2 (native sport structural model). While Research Question 1 pertained to a specific ethnic player, Research Question 2 related to the Native Sport Structural Model focused on the relationships among ethnic identity, acculturation, identification with a popular sport in a native country (IPSNC) and consumption for a popular sport in a native country (CPSNC). First, since ethnic identity was marginally and negatively correlated with acculturation, the finding supports Berry's bi-dimensional model of acculturation like RQ1.

Second, ethnic identity positively influenced IPSNC, but did not directly affect CPSNC. This finding can be partially understood by the concept of ethnocentrism. Ethnocentrism refers to the tendency of an individual to identify strongly with his/her own cultural/ethnic group. Shimp, Sharma, and Shin (1995) expanded the term ethnocentrism into the business context, using the term "consumer ethnocentrism." Ethnocentric consumers have strong intentions and willingness to favor domestic products over foreign products. To them, purchasing foreign products is bad, inappropriate, and unpatriotic. Therefore, respondents in the current study expressed their ethnic identity by identifying with a sport product possessing attributes of their native 
countries (a popular sport in a native country in this study). This finding is also consistent with previous studies which found people who had a stronger sense of ethnic identity were more likely to consume culture-specific food and attend culture-specific entertainment activities (Cleveland et al., 2009; Pons et al., 2001; Xu et al., 2004). Different from the concept of consumer ethnocentrism, however, ethnic identity did not directly influence CPSNC. One plausible explanation is that respondents in the current study reported very low levels of consumption for the native popular sports $(M=2.12)$. Considering people are actually limited to consuming popular sports in their native countries only through the Internet, this finding is understandable.

Although ethnic identity did not directly affect CPSNC, it indirectly influenced CPSNC through IPSNC since the identification variable in Research Question 2 (IPSNC) significantly influenced the consumption variable (CPSNC). In other words, Asians maintaining high levels of identification with their native county tend to consume a popular sport in a native country by identifying with the native popular sports. For example, Koreans living in the U.S. are likely to watch the Korean Baseball League through the Internet by identification with the sport.

The last finding relative to Research Question 2 is that, as expected, acculturation did not influence IPSNC or CPSNC indicating respondents were not likely to identify with and consume native popular sports as a means of adaptation to the host country. To sum up, similar to the Ethnic Player Model, the results of this model revealed acculturation and ethnic identity were marginally and negatively associated with one another, indicating that people with low levels of ethnic identity do not necessarily show high levels of acculturation. Ethnic identity positively influenced IPSNC, but did not 
directly influence CPSNC. However, ethnic identity indirectly influenced CPSNC by the influence of IPSNC on CPSNC. For acculturation, it did not affect both IPSNC and CPSNC.

Research Question 3 (host sport structural model). Research Question 3 pertaining to the Host Sport Structural Model addressed the relationships among ethnic identity, acculturation, identification with a popular sport in a host country (IPSHC), and consumption for a popular sport in a host country (CPSHC). First, since acculturation and ethnic identity were marginally and negatively correlated with one another, the results supported the bi-dimensional model of acculturation (Berry, 1990, 1997), indicating people with high ethnic identity do not necessarily show low levels of acculturation. Second, ethnic identity did not impact either IPSHC or CPSHC. This finding was expected from the perspective of the unidimensional model of acculturation which implied that if individuals are lowly identified with their native country, they would attach to and consume products/services reflecting attributes of the host country rather than those of the native country (Nguyen, Messe, \& Stollak, 1999). This finding seems to support prior studies in which ethnic identity for Hispanics (Harrole \&Trail, 2007) and Asians (Ha, Hums, \& Greenwell, 2011) did not significantly influence consumption of American football, the most popular sport in the U.S.

Third, acculturation positively influenced IPSHC, but did not directly affect CPSHC. This finding partially supports a prior study which found acculturation significantly influenced both American football identification and consumption (Ha, Hums, et al., 2011). However, the finding is contrast with another study which revealed acculturation positively and significantly affected consumption for a representative sport 
in a host country (ice hockey in the Canada; Pons et al., 2001). It is in line with the notion that ethnic minorities identify with and eventually consume popular sports in the host country in order to adapt well to the host society. However, the outcome of the study indicated that acculturation did not directly influence CPSHC. One primary explanation for this result is that even though Asians identified with the host country popular sports, they are not usually interested in consuming popular sports directly in the host country. Indeed, the sport consumption levels of Asians in the U.S. were significantly low compared to other ethnic minority groups, such as Hispanics and African Americans. According to Spanberg (2003), Asians represented less than $2 \%$ of total fans in each of the following five popular sports in the U.S.: (a) NFL (1.0\%), NBA (1.3\%), MLB (0.9\%), NHL (1.2\%), and NASCAR (0.7\%).

However, the findings imply that acculturation indirectly influenced CPSHC through IPSHC since IPSHC significantly influenced the consumption variable (CPSNC) in this Research Question. In other words, Asians adapting well to the U.S. are inclined to consume a popular U.S. sport (American football in this study) by identifying with the sport.

In summary, like the Ethnic Player and Native Sport models, acculturation was marginally and negative correlated with ethnic identity. Ethnic identity did not significantly influence either IPSHC or CPSHC. Acculturation positively affected IPSHC, but did not directly influence CPSHC. Similar to the previous two models, IPSHC positively influenced CPSHC. These findings imply that an Asian who is highly acculturated to the host country tends to consume the host's popular sport by identifying with the sport. For example, the four Asian ethnic groups in the current study (Chinese, 
Japanese, Korean, and Taiwanese) who were adapting well to U.S. society tended to watch American football by identifying with the sport.

The overall summary of key results and meanings pertaining to Research Question 1-3 is presented in Table 21 below.

Table 21.

Summary of Results and Meanings for Research Questions 1-3

\begin{tabular}{cll}
\hline $\begin{array}{c}\text { Research } \\
\text { Questions }\end{array}$ & \multicolumn{1}{c}{ Results } & \multicolumn{1}{c}{ Meanings } \\
\hline RQ 1 & $\begin{array}{l}\text { Ethnic identity indirectly } \\
\text { influenced CTEP through } \\
\text { IEP }\end{array}$ & $\begin{array}{l}\text { An Asian highly identified with his/her } \\
\text { native country is likely to watch a team's } \\
\text { games in which ethnic players are } \\
\text { playing (e.g., Ichiro Suzuki in Seattle } \\
\text { Mariners) by identifying with Ichiro } \\
\text { Suzuki. }\end{array}$ \\
\hline RQ 2 & $\begin{array}{l}\text { Ethnic identity indirectly } \\
\text { influenced CPSNC through } \\
\text { IPSNC }\end{array}$ & $\begin{array}{l}\text { An Asian highly identified with his/her } \\
\text { native country tends to consume a } \\
\text { popular sport in a native country by } \\
\text { identifying with the native sport (e.g., } \\
\text { Koreans living in the U.S. are likely to } \\
\text { watch Korean Baseball League through } \\
\text { the Internet by identification with the } \\
\text { sport. }\end{array}$ \\
\hline RQ 3 & $\begin{array}{l}\text { Asians adapting well to the U.S. are } \\
\text { influenced CPSHC through } \\
\text { IPSHC }\end{array}$ & $\begin{array}{l}\text { sport (American football in this study) by } \\
\text { identifying with the sport. }\end{array}$ \\
& &
\end{tabular}

Research Questions 4 (the impact of acculturation strategies on identifications

and consumptions). Research Question 4, including 4-1, 4-2, and 4-3, addressed the impact of the four acculturation strategies on three identifications (IEP, IPSNC, and IPSHC) and three consumptions (CTEP, CPSNC, and CPSHC). As noted in Chapter II, Berry $(1990,1997)$ developed four acculturation strategies based on the bi-dimensional 
model: (a) integration, (b) assimilation, (c) separation, and (d) marginalization. Looking at the characteristics of each strategy, people using the integration strategy tend to maintain values, identity, and behaviors of their native country, while at the same time accepting those of the host country. In contrast, those using the marginalization neither maintain values, identity, and behaviors of the native country nor accept those of the host country. Individuals using the assimilation strategy prefer to accept values, identity, and behaviors of the host country while not maintaining those of the native country. On the other hand, people using the separation strategy prefer to preserve cultural identity, values, and behaviors of the native country while having little or no interest in those of the host country.

Applying the characteristics of each acculturation strategy into a sport consumption context, it was expected that Asians using integration and separation strategies would be more likely to identify with and consume sport products possessing attributes of the native country (ethnic player and national popular sports in this study) than those in assimilation and marginalization strategies. In contrast, Asians in the integration and assimilation strategies would be more likely to identify with and consume sport products reflecting attributes of the host country (host popular sports in this study).

The results of the current study supported the above expectations. First, respondents using the integration and separation strategies were more identified with ethnic players and consumed than those in the other two strategies. In the same manner, those in the integration and separation strategies were more likely to identify with and consume national popular sports than those in the assimilation and marginalization strategies. Further, those in the integration and assimilation strategies identified with and 
consumed host popular sports greater than those in separation and marginalization. These findings directly support Maldonado and Tansuhaj's (2002) findings that individuals using integration and assimilation strategies bought the host country brands whengrocery shopping more often than those in the separation strategy, whereas those using the integration and separation strategies purchased the native country brand when grocery shopping more often than those using the assimilation strategy. Additionally, these findings are consistent with those of a recent study which found Asians using integration and assimilation strategies were more likely to identify with and consume American football, which is a representative American sport, than subjects using the other two strategies (Ha, Hums, \& Greenwell, 2011).

To summarize, the impact of acculturation strategies (integration, assimilation, separation, and marginalization) on IEP and CTEP (Research Question 4-1) was significant while controlling for the length of residence in the U.S. Specifically, when controlling for the effect of the length of residence, Asians using integration and separation strategies were more likely to identify with their ethnic player (e.g., Ichiro Suzuki, Shin-Soo Choo) and watch or attend a team's games with an ethnic player (e.g., Seattle Mariners, Cleveland Indians) than those using assimilation and marginalization strategies. The four acculturation strategies had significant impact on IPSNC and CPSNC when controlling for the length of residence (Research Question 4-2). Specifically, Asians using integration and separation strategies were more likely to identify with and consume native popular sports (e.g., Korean Baseball League, Nippon Professional Baseball) than those using the other two strategies, while controlling for the effect of the length of residence. The findings are the same as the Research Question 4-1. The impact 
of acculturation strategies on IPSHC and CPSHC was significant when controlling for the effect of length of residence (Research Question 4-3). Specifically, Asians using integration and assimilation strategies were more likely to identify with host popular sports (e.g., American football) and consume the host popular sports than those using the other two strategies. A summary of results and meanings pertaining to Research Question 4 is presented in Table 22 below.

Table 22.

Summary of Results and Meanings for Research Question 4

\begin{tabular}{|c|c|c|}
\hline $\begin{array}{l}\text { Research } \\
\text { Questions }\end{array}$ & Results & Meanings \\
\hline RQ 4-1 & $\begin{array}{l}\text { Significant difference in } \\
\text { IEP and CTEP among } \\
\text { acculturation strategies }\end{array}$ & $\begin{array}{l}\text { People using integration and separation } \\
\text { strategies were more likely to identify } \\
\text { with their ethnic player and consume a } \\
\text { team's games with ethnic player than } \\
\text { those using assimilation and } \\
\text { marginalization strategies. }\end{array}$ \\
\hline RQ 4-2 & $\begin{array}{l}\text { Significant difference in } \\
\text { IPSNC and CPSNC among } \\
\text { acculturation strategies }\end{array}$ & $\begin{array}{l}\text { People using integration and separation } \\
\text { strategies were more likely to identify } \\
\text { with and consume native popular sports } \\
\text { than those using the other two strategies. }\end{array}$ \\
\hline RQ 4-3 & $\begin{array}{l}\text { Significant difference in } \\
\text { IPSHC and CPSHC }\end{array}$ & $\begin{array}{l}\text { People using integration and assimilation } \\
\text { strategies were more likely to identify } \\
\text { with and consume host popular sports } \\
\text { than those using the other two strategies. }\end{array}$ \\
\hline
\end{tabular}

\section{Practical Implications}

Since the current U.S. sport industry is highly competitive and saturated, it is critical for sport marketers to develop new or niche markets to survive such a rough environment (Park, Mahony, \& Greenwell, 2010). Due to their tremendous economic power and rapid population growth, ethnic minority groups in the U.S. are promising 
niche markets for various sport organizations (Ha, Ha et al., 2011). In order for sport organizations to attract more ethnic minorities (Asians in this study), it was necessary to understand how they consume sports differently depending on their levels of acculturation and ethnic identity. By examining these two cultural factors, the results of the current study provide several meaningful implications for sport practitioners even if some implications may not be novel.

First of all, the results of the study revealed that ethnic identity primarily influenced respondents' perceptions of sport products possessing characteristics of their original culture (i.e., ethnic player playing in the U.S., native popular sports). The findings indicate Asians living in the U.S. tend to show a more favorable attitude toward culturalrelated sport products as a way of maintaining their ethnic identity. From this standpoint, U.S. sport marketers should put their best efforts to employ a culturally based marketing approach to effectively communicate with Asian consumers.

When it comes to ethnic players, for example, sport teams or leagues having Asian players need to provide friendlier multimedia platforms aimed at their specific target Asian subgroup. This may include translated versions of websites, broadcasting the team's games in a native language, or hiring native-language speaking staff. Indeed, when the Houston Rockets recruited Yao Ming in 2002, the team broadened its marketing efforts to attract Asian populations by broadcasting a weekly Chinese radio show, launching a Chinese-version of the team website, and employing Chinese-speaking staff (Clarke \& Mannion, 2006; Wang \& Zhang, 2009). Although the language-related strategy seems less appropriate for the U.S. born Asians who are more English dominant, King (2011) argued that they would also prefer to watch sports in their native language as 
long as it was culturally relevant. Such language based marketing strategies may also be utilized for sport teams when Asian players come to a city as members of the visiting team. Another possible marketing strategy is to provide various promotional events for Asian populations, such as Asian Community Appreciation Days and offering Asian food service. This is particularly important to professional sports teams located in big cities such as New York, Los Angeles, San Francisco, Houston, Dallas, and Chicago where many Asians reside. For instance, using the first name of Japanese baseball superstar, Ichiro Suzuki, the Seattle Mariners sold "Ichi Roll" sushi (tuna sushi sold at concession area of Safeco Field). Further, sport teams in these big cities, as a part of community relations programs, can utilize Asian players as a marketing and promotional tool by connecting them to a particular ethnic group community or cultural center (e.g., Korean community centers or Korean student associations in Cleveland).

While the above marketing strategies pertain to targeting Asians living in the U.S., Lizandra and Gladden (2005) and Ko et al. (2008) suggested that the presence of Asian players allows professional teams or leagues in the U.S. to expand their fan base and increase revenues by marketing them in their homelands. For example, broadcasting ethnic players' games, hosting exhibition games in their homelands, and collaboration with ethnic players' previous teams in the homeland may be practical marketing strategies. Although the target population in the current study was Asians living in the U.S., the results pertaining to ethnic players imply that sport teams with ethnic players need to target and market Asians in their homelands, along with Asians in the U.S.. Considering the saturation of the U.S. sports market and that Asian countries are a relatively underdeveloped sport market segment, it is a particularly lucrative option for 
sport organizations in the U.S. to expand their fan bases internationally by utilizing highprofile Asian athletes.

As the importance of ethnic players becomes apparent in terms of the marketing aspects of teams and even leagues, sport managers should pay attention to how ethnic players, mostly from foreign countries, adapt to a new country as well as to the new sport environment in the country. The degree of which foreign athletes (ethnic players) adapt to a new environment affects coaches, administrators, teammates, and even spectators. For instance, foreign athletes who do not adapt well to the new environment are more likely to leave, which can hurt team cohesion (Popp, 2007). Further, the loss of foreign athletes can also negate any competitive and marketing advantages gained by landing the foreign athletes.

Another type of cultural-related sport product examined in the current study was a popular sport in a native country. Due to the ever-increasing popularity of the Internet, cutting-edge digital and computerized technologies have emerged. Examples of these technologies include blogs, podcasts, Facebook, Twitter, YouTube, and smartphones. The evolution of new technologies derived from the Internet has eliminated geographic restrictions and distance (Filo, 2011). Put another way, regardless of where sport fans are, they can follow their favorite teams, players, coaches, and sporting events anywhere in the world via new media technologies based upon the Internet. This access seems particularly important for people living out of a native country either temporarily (e.g., international students) or permanently (e.g., immigrants). Thus, even though they are living in a host society, they can still follow their favorite sports, teams, players, or coaches in their native countries and identify with them through the Internet. 
Respondents of the current study indicated that they had very low levels of consumption for the native popular sports $(M=2.12)$. From the perspective of native sport marketers (e.g., sport marketers in Korea), this means that Asians (Koreans) living in the U.S. are considered an underdeveloped market segment. Sport marketers in the native countries, therefore, may need to develop specific marketing strategies for people living abroad in the U.S. or another country in order to attract fans.

Second, the results of the study indicated the acculturation factor primarily influenced respondents' perceptions of sport products possessing characteristics of the host country (i.e., a popular sport in the host country). This finding indicates highly acculturated Asians living in the U.S. tend to show more favorable attitudes toward host country sport products reflecting attributes of the host country as a way of adapting to the U.S.. Even though it seems that sport marketers do not have to utilize cultural specific marketing strategies with regard to popular sports in the U.S., Alvaro Saralegui, former Sports Illustrated general manager, emphasized the importance of bicultural marketing strategy for the U.S. sports:

There is a growth in the love of American sport as acculturation increases. What we want to do is accelerate that growth. Instead of going to the English dominant, focus on the bicultural dominants... who are leaning toward becoming more bicultural (King, 2011, p. 1).

Therefore, sport marketers may need to employ both cultural specific and American style marketing strategies to attract Asians. These bilingual and bicultural marketing strategies are especially useful for the U.S. born Asians because they tend to be influenced by ethnic identity from their parents as well as by American identity from 
their U.S. peers. For recent immigrants who may have language barriers, a previous study found that language issues and lack of host country friends prevented Chinese people from accessing information about popular sports in a host country, even though they were interested in the sports to help them acculturate into a new society (Hudson, Hinch, Walker, \& Simpson, 2010). Thus, recent Asian immigrants may be more persuaded to consume popular U.S. sports in a comfortable learning environment where they can have an opportunity to become familiar with the sports. Sport marketers need to focus more on education. For instance, given American football is considered an unfamiliar sport to most recent Asian immigrants, introducing game rules and various promotional information about them would be worth considering.

The last practical implication pertains to the four acculturation strategies: (a) integration, (b) assimilation, (c) separation, and (d) marginalization. The results indicated Asians using integration and separation strategies were more likely to identify with and consume sport products possessing attributes of the native country (ethnic player and national popular sports in this study) than those using assimilation and marginalization strategies. In contrast, Asians using integration and assimilation strategies were more likely to identify with and consume sport products reflecting attributes of the U.S. (host popular sports in this study). These findings indicated Berry's four acculturation strategies would play a significant role in segmenting Asian sport consumers. In other words, Berry's model can be utilized as a new tool for market segmentation. Market segmentation divides a market into categories of people with identifiable and similar characteristics. From the perspective of sport marketers, therefore, Berry's four acculturation strategies can be utilized as a new tool to segment Asian consumers in a 
sport context. This might be a particularly useful segmentation tool to expand a team's fan base by identifying Asians and other ethnic minority groups as niche markets. Within each segment (strategy), individuals' attitudes and behaviors toward either national sports or host sports were similar, indicating a target market that would react similarly to a marketing plan. Such an approach would allow sport marketers to determine which segments are reachable and profitable, and design effective marketing plans targeting the desired segment(s).

Regarding cultural-related sport products (e.g., ethnic players, popular sports in a native country), for Asians using either integration or separation strategies, sport managers should develop and implement cultural specific marketing and promotional strategies based on Asian fans' desires and needs. Further, those using the integration and separation strategies should be considered primary target groups as opposed to those using assimilation and marginalization. Just like famous museums, one practical marketing strategy sport teams with ethnic players can utilize for the two target groups is to provide a specific Asian language service to serve a designated target market by offering a full Asian language radio broadcast for a home game or using designated native-language speaking commentators. Specifically, the teams may need to distribute multi-language adapters or translation headsets for a targeted Asian group at a stadium entrance (e.g., Japanese translation headset at a Seattle Mariners game). Further, providing subtitles in a designated language for in-stadium announcements on electronic scoreboards or Jumbotrons may be practical strategies to draw interests of Asians.

With regard to sport products reflecting attributes and characteristics of the U.S. (popular sports in the U.S.), it is appropriate for sports marketers to develop and 
implement analogous marketing and promotional strategies to target Asians using the integration and assimilation strategies in that those using the two strategies are inclined to accept behaviors, identity, or values of people from the U.S.. On the other hand, marketers may need to develop cultural-specific promotional strategies (e.g., Asian Community Day) for the needs and desires of Asians who are using the separation strategy because they are likely to maintain behaviors or values of their original culture.

\section{Future Research}

While the examination of psycho-cultural factors (acculturation, ethnic identity) is still in its initial stage in the sport marketing literature, the current study establishes an important step in understanding the role of these two psycho-cultural factors for Asians' sport consumption behaviors. The three sport consumption models tested in the current study examined the relationships among the two cultural factors (acculturation and ethnic identity), identification, and sport consumption. Future research could include more socio-demographic variables in the models. The literature on acculturation and sport consumption has shown that gender, age in migration, length of residence in a host country, and so forth, influence one's level of acculturation as well as sport consumption behaviors in a host country. For example, the longer an individual resides in the host country, the higher his/her level of acculturation. (Berry, 1997), which may in turn result in consuming products or services reflecting attributes of the host society. Although the current study tested the length of residence variable in examining acculturation strategies for the second purpose (the impact of acculturation strategies on identifications and consumptions), the variable was excluded in the three consumption models since the main purpose of this study was to test and develop sport consumption models rather than 
testing moderating effect of socio-demographic variables (e.g., the length of residence). Including some important socio-demographic variables in the current sport consumption models would result in more robust and rigorous models.

Future studies should also thoroughly examine how socializing agents (e.g., family members, peer groups, mass media), along with the two cultural factors (acculturation and ethnic identity), influence sport consumption behaviors for Asians in the U.S. Prior research found that Asian young adults were more likely to participate in culture-specific consumption behaviors when they were with the same ethnic friends than when were with Caucasian friends (Xu, et al., 2004). In addition, the lack of friends was found to be a major constraint for Chinese people wanting to participate in sports in Canada (Hudson et al., 2010). Therefore, future studies can help determine the moderating role of socializing agents in the relationship between the two cultural factors and sport consumption behaviors.

While the current study focused on the four specific Asian ethnic subgroups (Chinese, Japanese, Korean, and Taiwanese), the results of the current study cannot be generalized to other Asian subgroups. According to Kaufman-Scarborough (2000), making generalizations of certain beliefs regarding one or a few Asian Americans to the whole Asian American population can become a marketing problem. Asian Americans come from more than 30 different ethnic subgroups, each of which has its own culture. Therefore, future research needs to confirm the impact of acculturation and ethnic identity on sport consumption behaviors by collecting data from other Asian subgroups. Doing so would allow researchers and marketers to detect differences and similarities in sport consumption behaviors among various subgroups. For example, even though the 
four Asian subgroups of the current study (e.g., Chinese, Japanese, Korean, and Taiwanese) are combined as a similar categorization based on geographical closeness (Magazine Publishers of America, 2004), each of the four groups has its own specific cultural characteristics.

The next suggestion for future research would be to consider the effect of Asian athletes on sport sponsorship and endorsements. The expanding market of ethnic sport consumers is leading more and more companies to invest the significant amounts of money in corporate sponsorship. In particular, today's rapid increase in the number of Asian athletes in the North American professional sport leagues would stimulate the interests of corporations in using sport sponsorship and endorsements to target Asian populations (McKelvey, 2011). Hence, future research needs to investigate the importance of the ethnic background of athletes in the fields of sport sponsorship and athlete-endorsement.

Although some previous studies (e.g., Ha, et al., 2011; Ha, Hums et al.,2011; Harrole \& Trail, 2007; Pons et al., 2001;) have acknowledged that the construct of acculturation helps sport marketers understand various sport consumption behaviors of ethnic minority groups, no study has paid particular attention to developing an acculturation scale for ethnic groups in a sport context. Therefore, to better understand various sport consumption behaviors for ethnic minorities, it is inevitable to develop a valid and reliable measurement for acculturation in a sport setting. This would contribute to establishing a robust body of knowledge for sport consumption behavior of ethnic minority groups. 
Another suggestion for future research is related to Berry's four acculturation strategies. It is plausible that the degree of identification with and consumption of a certain same sport (e.g., basketball) between a native country and host country may be different according to acculturation strategies. For example, Koreans would follow the native country's professional league for a particular sport [e.g., Korean Basketball League (KBL)] in separation strategy, follow the host country's league of the sport (e.g., MLB) in assimilation strategy, follow both leagues in integration strategy, or fail to follow either league in marginalization.

Finally, it would be interesting to expand the concept of acculturation to not only ethnic minority individuals, but also to any sports fans moving to or from other provinces, states, or even cities. Therefore, exploring the concept of acculturation in a sport context to target migrating sports fans to other regions within a nation may assist sports teams to develop new fan base and/or maintain and engage their existing fans.

\section{Summary of the Study}

In summary, the current study developed and tested three Sport Consumption Models for Asians living in the U.S. based on ethnic identity and acculturation. Specifically, each of the three consumption models was differentiated based upon the types of identifications (IEP, IPSNC, and IPSNC) and consumption behaviors (CTEP, CPSNC, and CPSHC). The overall results revealed ethnic identity primarily influenced cultural-specific sport products (ethnic player and a popular sport in a native country), while acculturation primarily influenced sport products reflecting attributes of the host country (the U.S.). 
The current study also examined the impact of the four acculturation strategies (integration, assimilation, separation, and marginalization) on the three types of identifications (IEP, IPSNC, and IPSNC) and consumption behaviors (CTEP, CPSNC, and CPSHC). Results indicated Asians using integration and separation strategies were more likely to identify with and consume cultural-specific sport products (ethnic player and national popular sports in this study) than those using assimilation and marginalization strategies. In contrast, Asians using integration and assimilation strategies were more likely to identify with and consume sport products reflecting attributes of the U.S. (popular sports and host popular sports in this study).

Theoretically, the current study extends the literature on ethnic minorities' sport consumption behavior, by incorporating the two cultural factors, ethnic identity and acculturation. Overall, the findings confirmed the theoretical suppositions based on the notion of ethnic identity and acculturation. Such a theoretical foundation to explain the role of ethnic identity and acculturation is a significant contribution to the body of literature on ethnic minority sport consumption behavior. While the previous literature on acculturation and ethnic identity in a sport context has primarily focused on participant sport settings, the current study expanded the scope of research into spectator sport settings. In addition, this study also provides meaningful insights and information for sport marketers into understanding Asian's sport consumption behaviors, an underexamined ethnic minority group in the sport consumption behavior literature.

Practically, the results of the study indicated that sport marketers should develop marketing and promotional strategies that are tasteful and culturally appropriate for Asians in regard to cultural-specific products, while developing bicultural marketing 
strategies regarding host country sport products. In addition, Berry's $(1990,1997)$ four acculturation strategies employed in the current study could be utilized as a new tool for market segmentation in a sport context. 


\section{REFERENCES}

Armstrong, K. L. (1998). Ten strategies to employ when marketing sport to black consumers. Sport Marketing Quarterly, 7(3), 11-18.

Armstrong, K. L. (2002a). An examination of the social psychology of Blacks' consumption of sport. Journal of Sport Management, 25, 267-288.

Armstrong, K. L. (2002b). Race and sport consumption motivations: A preliminary investigation of a Black consumers' sport motivation scale. Journal of Sport Behavior, 25, 309-330.

Armstrong, K. L., \& Peretto Stratta, T. M. (2004). Market analyses of race and sport Consumption, Sport Marketing Quarterly, 13, 7-16.

Ary, D., Jacobs, L., \& Razavieh, A. (2002). Introduction to research in education (6th ed.). Belmont, CA: Wardsworth.

Asian-American Market in the U.S. (2008). Rockville, MD: Packaged Facts.

Asian-Nation (n. d.). Welcome to Asian-Nation. Retrieved from http://www.asiannation.org/index.shtml

Assael, H. (1992). Consumer behavior and marketing action (4th ed.). Boston, MA: PWS-Kent.

Babbie, E. R. (2007). The practice of social research (11th ed.). Belmont, CA: Thomson Learning. 
Barry, D. T. (2001). Development of a new scale for measuring acculturation: The East Asian Acculturation Measure (EAAM). Journal of Immigrant Health, 3(4), 193197.

Bates, R. A. (2005). Multivariate research methods. In R. Swanson \& E. Holton III (Eds.), Research in organizations: Foundations and methods of inquiry (pp. 115142). San Francisco, CA: Berrett-Koehler.

Beiser, M. M., Barwick, C., Berry, J.W., da Costa, G., Fantino, A., Ganesan, S., Lee, C., Milne, W., Naidoo, J., Prince, R., Tousignant, M., \& Vela, E. (1988). Mental health issues affecting immigrants and refugees. Ottawa, Canada: Health and Welfare Canada.

Berry, J. W. (1980). Acculturation as varieties of adaptation. In. A. M. Padilla (Ed.). Acculturation: Theory models, and some new findings (pp. 9-25). Boulder, CO: Westview.

Berry, J. W. (1990). Psychology of acculturation: Understanding individuals moving between cultures. In R. W. Brislin (Ed.), Applied cross cultural psychology (pp. 232-253). Newbury Park, CA: Sage.

Berry, J. W. (1997). Immigration, acculturation, and adaptation. Applied Psychology: An International Review, 46(1), 5-34.

Berry, J. W. (2003). Conceptual approaches to acculturation. In J. W. Berry, K. M. Chun, P. Balls Organista \& G. Martin (Eds.), Acculturation: Advances in theory, measurement, and applied research. (pp. 17-37). Washington, DC: American Psychological Association. 
Bilyeu, J. K., \& Wann, D. L. (2002). An investigation of racial differences in sport fan motivation. International Sports Journal, 6(2), 93-106.

Boeckner, L. S., Jordan, S. A., \& Schnepf, M. I. (2000). The use of an ethnic food frequency questionnaire among Hispanic women. Great Plains Research, 10(2). 295-304

Branscombe, N. R., \& Wann, D. L. (1991). The positive social and self concept consequences of sports team identification. Journal of Sport \& Social Issues, 15(2), 115-127.

Brenner, S. (2004, May 31-June 6). A world of opportunity: Teams see potential for big payday by taking their message to different cultures. SportsBusiness Journal, 15 19.

Brislin, R. W. (1970). Back-translation for cross-cultural research. Journal of CrossCultural Psychological, 1, 185-216.

Browne, M. W., \& Cudeck, R. (1992). Alternatives ways of assessing model fit. Sociological Methods and Research, 21, 230-258.

Castro, V. S. (2003). Acculturation and psychological adaptation. Wesport, CT: Greenwood Press.

Choi, A. (2010). The impact of ethnic diversity on the Ladies Professional Golf Association: A case study of Anheuser-Busch and its sponsorship objectives and strategies. Sport Marketing Quarterly, 19, 51-57.

Chou, C. P., \& Bentler , P. M. (1995). Estimates and tests in structural equation modeling. In R. H. Hoyle (Ed.), Structural equation modeling: Concepts, issues, and applications (pp. 37-55). Thousand Oaks, CA: Sage. 
Chung, R. H. G., Kim, B. S. K., \& Abreu, J. M. (2004). Asian American multidimensional acculturation scale: Development, factor analysis, reliability, and validity. Cultural Diversity \& Ethnic Minority Psychology, 10(1), 66-80.

Clarke III, I., \& Mannion, R. (2006). Marketing sport to Asian-American consumers. Sport Marketing Quarterly, 15, 20-28.

Claussen, C. L., Ko, Y. J., \& Rinehart, R. E. (2008). Cultural diversity: An alternative approach to understanding sport participant consumer behavior. The SMART Journal, 4(2), 58-71.

Cleveland, M., \& Laroche, M. (2007). Acculturation to the global consumer culture: Scale development. Journal of Business Research, 60(3), 249-259.

Cleveland, M., Laroche, M., Pons, F., \& Kasto, R. (2009). Acculturation and consumption: Textures of cultural adaptation. International Journal of Intercultural Relations, 33(3), 196-212.

Cohen, J. (1988). Statistical power analysis for the behavioral sciences (2nd ed.). Hillsdale, NJ: Erlbaum.

Cronk, B. C., \& West, J. L. (2002). Personality research on the Internet. A comparison of web-based and traditional instruments in take-home and in-class settings. Behavior Research Methods, Instruments, \& Computers, 34, 177-180.

Cuellar, I., Arnold, B., \& Maldonado, R. (1995). Acculturation rating scale for Mexican American-II: A revision of the original ARSMA Scale. Hispanic Journal of Behavioral Sciences, 17, 275-304. 
Cuellar, I., Nyberg, B., Maldonado, R. E., \& Roberts, R. E. (1997). Ethnic identity and acculturation in a young adult Mexican-origin population. Journal of Community Psychology, 25, 535-549.

Curry, L., \& Jackson, J. (2003). The science of inclusion: Recruiting and retaining racial and ethnic elders in health research. Washington, DC: The Gerontological Society of American.

Davis, M. C. (2009). Analyzing the relationship between team success and MLB attendance with GARCH effects. Journal of Sports Economics, 10(1), 44-58.

DeSchriver, T. D., \& Jensen, P. E. (2002). Determinants of spectator attendance at NCAA Division II football contests. Journal of Sport Management, 16, 311-330.

DeVellis, R. F. (2003). Scale development: Theory and applications. Thousand Oaks, CA: Sage Publications.

Diamantopoulos, A., \& Winklhofer, H.M. (2001). Index construction with formative indicators: An alternative to scale development. Journal of Marketing Research, $38,269-277$.

Dietz-Uhler, B. \& Lanter, J. (2008). The consequences of sports fan identification. In L. W. Hugenberg, P. M. Haridakis, \& A. C. Earnheardt (Eds.), Sports mania: Essays on fandom and the media in the 21st century (pp. 103-113). Jefferson, NC: McFarland and Company.

Dillman, D. (2007). Mail and internet surveys: The tailored design method. New York: Wiley.

Donna, G., \& Berry, J. W. (1994). Acculturation attitudes and acculturation stress of Central American refugees. International Journal of Psychology, 29, 57-70. 
Erikson, E. (1968). Identity: Youth and crisis. New York: Norton.

Faul, F., Erdfelder, E., Lang, A.-G., \& Buchner, A. (2007). G*Power 3: A flexible statistical power analysis for the social, behavioral, and biomedical sciences. Behavior Research Methods, 39, 175-191.

Field, A. (2009). Discovering statistics using SPSS (3rd ed.). Thousand Oaks, CA: Sage Publications.

Filo, K. (2011). Sport and new media. In L. P. Masteralexis, C. A., Barr., \& M. A. Hums (Eds.), Principles and practice of sport management (4th ed., pp. 442-459). Sudbury, MA: Jones and Bartlett Learning.

Fink, J. S., \& Parker, H. M. (2009). Spectator Motives: Why do we watch when our favorite team is not playing? Sport Marketing Quarterly, 18(4), 210-217.

Fink, J. S., Trail, G. T., \& Anderson, D. F. (2002a). An examination of team identification: Which motives are most salient to its existence? International Sports Journal, 6(2), 195-207.

Fink, J. S., Trail, G. T., \& Anderson, D. F. (2002b). Environmental factors associated with spectator attendance and sport consumption behavior: Gender and team differences. Sport Marketing Quarterly, 11, 8-19.

Fisher, R. J., \& Wakefield, K. (1998). Factors leading to group identification: A field study of winners and losers. Psychology \& Marketing, 15, 23-40.

Fornell, C., \& Larcker, D. (1981). Evaluating structural equation models with unobservable variables and measurement error. Journal of Marketing Research, $18(1), 39-50$. 
Funk, D. C., \& James, J. (2001). The psychological continuum model: A conceptual framework for understanding an individual's psychological connection to sport. Sport Management Review, 4(2), 119-150.

Funk, D.C., \& James, J.D. (2006). Consumer loyalty: The meaning of attachment in the development of sport team allegiance. Journal of Sport Management, 20(2), 189217.

Funk, D.C., Mahony, D. F., Nakazawa, M., \& Hirakawa, S. (2001). Development of the sport interest inventory (SII): Implications for measuring unique consumer motives at team sporting events. International Journal of Sports Marketing \& Sponsorship, 3, 291-316.

Funk, D. C., Mahony, D. F., \& Ridinger, L. L. (2002). Characterizing consumer motivation as individual difference factors: augmenting the Sport Interest Inventory (SII) to explain level of spectator support. Sport Marketing Quarterly, $11(1), 33-43$.

Gentry, J. W., Jun. S., \& Tansuhaj, P. (1995). Consumer acculturation processes and cultural conflict: How generalizable is a North American model for marketing globally? Journal of Business Research, 32, 129-139.

George, D., \& Mallery, P. (2001). SPSS for windows step by step: A simple guide and reference 10.0 update (3rd ed.). Boston: Allyn \& Bacon.

Gouke, C. G. (1987). Blacks and the American economy. Needham Heights, MA: Ginn. Ghuman, P. (1997). Assimilation or integration? A study of Asian adolescents. Educational Research, 39(1), 23-35. 
Gladden, J. M., Milne, G. R., \& Sutton, W. A. (1998). A conceptual framework for assessing brand equity in Division I college athletics. Journal of Sport Management, 12, 1-19.

Groves, R. M., Fowler, F. J., Couper, M. P., Lepkowski, J. M., Singer, E., \& Tourangeau, R. (2004). Survey methodology. Hoboken, NJ: Wiley.

Ha, J. P., Ha, J. H., \& Greenwell, T. C. (2011, October). Acculturation and ethnic identity in spectator sports: The linkages among acculturation, ethnic identity, points of attachment, and consumption behavior. Paper presented at the 2011 Sport Marketing Association (SMA) Conference, Houston, TX.

Ha, J. P., Hums, M. A., \& Greenwell, T. C. (2011). The impact of acculturation and ethnic identity on American football identification and consumption among Asians in the United States. Manuscript submitted for publication.

Hair, J. F., Anderson, R. E., Tatham, R. L., \& Black, W. C. (1998). Multivariate data analysis (5th ed.). Upper Saddle River, NY: Prentice Hall.

Hancock, D. R., \& Flowers, C. P. (2000). Social desirability responding on World Wide Web and paper-administered surveys. Paper presented at the national Convention of the Association for Educational Communications and Technology, Denver, Colorado.

Harrison, F. (2011, January 3). Re: MANOVA and correlations between dependent variables: How strong is too strong? [Online forum comment]. Retrieved from http://stats.stackexchange.com/questions/5926/manova-and-correlationsbetween-dependent-variables-how-strong-is-too-strong 
Harrole, M. G., \& Trail, G. T. (2007). Ethnic identification, acculturation and sports identification of Latinos in the United States. International Journal of Sports Marketing \& Sponsorship, 8, 234-253.

Harrole, M., \& Trail, G. T. (2010). Conative loyalty of Latino and non-Latino professional baseball fans. Journal of Sport Management, 24, 456-471.

Heere, B., \& James, J. D. (2007). Sports teams and their communities: Examining the influence of external group identities on team identity. Journal of Sport Management, 21, 319-337.

Hogg, M.A., \& Abrams, D. (1990). Social identity and social cognition: Historical background and current trends. In D. Abrams \& M.A. Hogg (Eds.) Social identity and social cognition (pp. 1-25), Malden, MA: Blackwell.

Hu, L., \& Bentler, P. M. (1999). Cutoff criteria for fit indexes in covariance structure analysis: Conventional criteria versus new alternatives. Structural Equation Modeling, 6, 1-55.

Hudson, S., Hinch T., Walker, G., \& Simpson, B. (2010). Constraints to sport tourism: A cross-cultural analysis. Journal of Sport and Tourism, 15(1), 71-88.

Humphreys, J. M. (2009). The multicultural economy 2009. Retrieved from http://www.terry.uga.edu/selig/buying_power.html

Hunt, K. A., Bristol, T., \& Bashaw, R. E. (1999). A conceptual approach to classifying sports fans. Journal of Services Marketing, 13(6), 439-452.

James, J., \& Trail, G. T. (2008). The relationship between team identification and sport consumption intentions. International Journal of Sport Management, 9, 427-440. 
Jang, Y., Kim, G., Chiriboga, D., \& King-Kallimanis, B. (2007). A bidimensional model of acculturation for Korean American older adults. Journal of Aging Studies, $21(3), 267-275$.

Jayasuriya, L., Sang, D., \& Fielding, A. (1992). Ethnicity, immigration and mental illness: A critical review of Australian research. Canberra, Australia: Bureau of Immigration Research.

Joreskog, K. G., \& Sorbom, D. (1998). LISREL 8: User's reference guide. Chicago: Scientific Software International.

Jun, S., Ball, A. D., \& Gentry, J. W. (1993). Modes of consumer acculturation. Advances in Consumer Research, 20(1), 76-82.

Kang, J., \& Kim, Y. K. (1998). Ethnicity and acculturation: Influences on Asian American consumers' purchase decision making for social clothes. Family and Consumer Sciences Research Journal, 27(1), 91-117.

Kaufman-Scarborough, C. (2000). Asian-American consumers as a unique market segment: Fact or fallacy? Journal of Consumer Marketing, 17(3), 249-262.

Keefe, S., \& Padilla, A. (1987). Chicago ethnicity. Albuquerque, NM: University of New Mexico Press.

Kim, B. S. K., \& Abreu, J. M. (2001). Acculturation measurement: Theory, current instruments, and future directions. In J. G. Ponterotto, M. Casas, L. A. Suzuki, \& C. Alexander (Eds.), Handbook of multicultural counseling (2nd ed.). (pp. 394424). Thousand Oaks, CA: Sage.

Kim. U. (1988). Acculturation of Korean immigrants to Canada. Unpublished Doctoral Dissertation, Queen's University. Canada. 
Kim, K., \& Cheong, Y. (2011). The effects of athlete-endorsed advertising: The moderating role of the athlete-audience ethnicity match. Journal of Sport Management, 25(2), 143-155.

Kim, C., Laroche, M., \& Joy, A. (1990). An empirical study of the effects of ethnicity on consumption patterns in a bi-cultural environment. In T. Kinnear (Ed.), Advances in consumer research (Vol. 17, pp. 839-846). Provo, UT: Association for Consumer Research.

Kim, Y. K., \& Trail, G. (2011). A conceptual framework for understanding relationships between sport consumers and sport organizations: A relationship quality approach. Journal of Sport Management, 25, 57-69.

King, B. (2007, January 22-28). Learning the culture: Successful Hispanic marketing goes well beyond language. SportsBusiness Journal, 10, pp. 14, 24.

King, B. (2011, July 18-22). The history behind numbers: What you need to know about the soaring growth of Hispanics and how to reach them. Retrieved from http://www.sportsbusinessdaily.com/Journal/Issues/2011/07/18/InDepth/Hispanics.aspx $? \mathrm{hl}=$ segment $\& s c=0$

Kline, R. B. (2005). Principles and practice of structural equation modeling (2nd ed.). New York: Guilford Press.

Ko, Y. J., Claussen, C. L., Rinehart, R. E., \& Hur, Y. (2008). Marketing to Asian American sport consumers. International Journal of Sport Management, 9(1), 67-80.

Krosnick, J. A., \& Fabrigar, L. R. (1997) Designing rating scales for effective measurement in surveys. In L. Lyberg, P. Biemer, M. Collins, E. de Leeuw, C. 
Dippo, N. Schwarz \& D. Trewin (Eds.), Survey measurement and process quality (pp. 141-164). New York: Wiley InterScience.

Kwon, H., \& Trail, G. (2001). Sport fan motivation: A comparison of American students and international students. Sport Marketing Quarterly, 10(3), 147-155.

Laosa, L. M. (1984). Social policies toward children of diverse ethnic, racial, and language groups in the United States. In H. W. Stevenson \& A. Siegel (Eds.). Child development research and social policy (pp. 1-109), Chicago: University of Chicago Press.

Landrine, H., \& Klonoff, E. A. (1994). The African American Acculturation Scale: Development, reliability, and validity. Journal of Black Psychology, 20, 104-127.

Lapchick, R. E. (2008, August 25). Games could have lasting impact for AsianAmericans. Street \& Smith's Sports Business Journal. Retrieved from http://www.sportsbusinessjournal.com/article/59861

Lapchick, R., Kaiser, C., Russell, C., \& Welch, N. (2010). The 2010 racial and gender report card: National Basketball Association. Retrieved from http://www.tidesport.org/RGRC/2010/2010_NBA_RGRC\%5B1\%5D.pdf

Lapchick, R., Kaiser, C., Caudy, D., \& Wang, W. (2010). The 2010 racial and gender report card: Major League Baseball. Retrieved from http://web.bus.ucf.edu/documents/sport/2010_MLB_RGRC.pdf

Lapchick, R., Kitnurse, J. M., Moss II, A. (2010). The 2010 racial and gender report card: National Football League. Retrieved from http://www.tidesport.org/RGRC/2010/2010\%20NFL\%20Racial\%20and\%20Gen der\%20Report\%20Card.pdf 
Le, C.N. (2010a). 14 important statistics about Asian Americans: The landscape of Asian America. Retrieved http://www.asian-nation.org/14-statistics.shtml

Le, C. N. (2010b). The 1965 immigrant Act: The landscape of Asian America. Retrieved http://www.asian-nation.org/1965-immigration-act.shtml

Leao, T. S., Sundquist, J., Johansson, S., \& Sundquist, K. (2009). The influence of age at migration and length of residence on self-rated health among Swedish immigrants: A cross-sectional study. Ethnicity \& Health, 14(1), 93-105.

Lee, W. N.,\& Um, K. R. (1992). Ethnicity and consumer product evaluation: A cross cultural comparison of Korean immigrants and Americans. Advances in Consumer Research, 19, 429-436.

Lee, Y. (2005). A new voice: Korean American women in sports. International Review for the Sociology of Sport, 40(4), 481-495.

Lizandra, M., \& Gladden, J. M. (2005). International sport. In L. P. Masteralexis, C. A., Barr., \& M. A. Hums (Eds.), Principles and practice of sport management $\left(2^{\text {nd }}\right.$ ed., pp. 166-194). Sudbury, MA: Jones and Bartlett Learning.

Magazine Publishers of America. (2004). Asian-American market profile. Retrieved from http://www.magazine.org/ASSETS/BF4E8BCE5E9D4847BA537A448EE20EF4 market_profile_asian.pdf

Mahony, D.F., Nakazawa, M., Funk, D.C., James, J.D., \& Gladden, J.M. (2002). Motivational factors influencing the behavior of J. League spectators. Sport Management Review, 5, 1-24.

Marcia, J. (1966). Development and validation of ego-identity status. Journal of Personality and Social Psychology, 3, 551-558. 
Marcia, J. (1980). Identity in adolescence. In J. Anderson (Ed.), Handbook of adolescent psychology (pp. 159-187). New York: Wiley.

Maldonado, R., \& Tansuhaj, P. (2002). Segmenting a local Latino market using Berry's acculturation taxonomy. Advances in Consumer Research, 29, 414-420

Matsuoka, H., \& Fujimoto, J. (2002, May). Foci of fan's psychological commitment. Paper presented at the meeting of the North American Society for Sport Management, Canmore, AB, Canada.

McCarthy, L. M. (1998). Marketing sport to Hispanic consumers. Sport Marketing Quarterly, 7(4), 19-24.

McCracken, G. (1986). Culture and consumption: A theoretical account of the structure and movement of the cultural meaning of consumer goods. Journal of Consumer Research, 13, 71-84.

McKelvey, S. (2011). Sport sponsorship. In L. P. Masteralexis, C. A., Barr., \& M. A. Hums (Eds.), Principles and practice of sport management (4th ed., pp. 442459). Sudbury, MA: Jones and Bartlett Learning.

Mendoza, R. H. (1989). An empirical scale to measure type and degree of acculturation in Mexican-American adolescents and adults. Journal of Cross-Cultural Psychology, 20(4), 372-385.

Min, P. G., \& Kim, Y. O. (2009). Ethnic and sub-ethnic attachments among Chinese, Korean, and Indian immigrants in New York City. Ethnic \& Racial Studies, 32, 758-780.

Moschis, G. P., \& Churchill, G. A. (1978). Consumer socialization: A theoretical and empirical analysis. Journal of Marketing Research, 15, 599-609. 
Netemeyer, R. G., Bearden, W. O., \& Sharma, S. (2003). Scaling procedures. Thousand Oaks, CA: Sage Publications.

Nguyen, H. H., Messe, L. A., \& Stollak, G. E. (1999). Toward a more complex understanding of acculturation and adjustment: Cultural involvements and psychological functioning in Vietnamese youth. Journal of Cross-Cultural Psychology, 30(5), 5-31.

Nguyen, H. H., \& von Eye, A. (2002). The acculturation scale for Vietnamese adolescents (ASVA): A bidimensional perspective. International Journal of Psychology, 20(1), 5-31.

Nunnally, J. C., \& Bernstein, I. H. (1994). Psychometric theory (3rd ed.) New York: McGraw Hill.

Oh, Y., Koeske, G., \& Sales, E. (2002). Acculturation, stress, and depressive symptoms among Korean immigrants in the United States. Journal of Social Psychology, 142(4), 511-516.

O’Guinn, T. C., Lee, W. N., \& Faber, R. J. (1986). Acculturation: The impact of divergent paths on buyer behavior. Advances in Consumer Research, 13, 579583.

Padilla, A. M. (1980). The role of cultural awareness and ethnic loyalty in acculturation. In A. M. Padilla (Ed.), Acculturation: Theory, models, and some new findings. Boulder, CO: Westview.

Padilla, A. M., \& Perez, W. (2003). Acculturation, social identity, and social cognition: A new perspective. Hispanic Journal of Behavioral Sciences, 25(1), 35. 
Park, S. H., Andrew, D. P. S., \& Mahony, D. F. (2008). Exploring the relationship between trait curiosity and initial interest in sport spectatorship. International Journal of Sport Management, 9, 286-302.

Park, S. H., Mahony, D. F., \& Greenwell, T. C. (2010). The measurement of sport fan exploratory curiosity. Journal of Sport Management, 24, 434-455.

Park, S., Paik, H. Y., J. D., Skinner, Ok , S.W., \& Spindler, A. A. (2003). Mother's acculturation and eating behaviors of Korean American families in California, Journal of Nutrition Education and Behavior 35(3): 142-147.

Persky, I., \& Birman, D. (2005). Ethnic identity in acculturation research: A study of multiple identities of Jewish refugees from the former Soviet Union. Journal of Cross-Cultural Psychology,36, 557-572.

Penaloza, L. (1994). Atravesando Fronteras/Border Crossings: A critical ethnographic exploration of the consumer acculturation of Mexican immigrants. Journal of Consumer Research, 21, 32-54.

Persons, S. (1987). Ethnic studies at Chicago: 1905-45. Urbana, IL: University of Illinois Press.

Phinney, J. S. (1989). Stages of ethnic identity development in minority group adolescents. The Journal of Early Adolescence, 9, 34-49.

Phinney, J. S. (1990). Ethnic identity in adolescents and adults: Review of research. Psychological Bulletin, 108(3), 499-514.

Phinney, J. S. (1992). The multigroup ethnic identity measure: A new scale for use with diverse groups. Journal of Adolescent Research, 7(2), 156-176. 
Phinney, J. S. (1996). When we talk about American ethnic groups, what do we mean? American Psychologist, 51(9), 918-927.

Phinney, J. S. (2003). Ethnic identity and acculturation. In K. Chun, P. Organista \& G. Martin (Eds.), Acculturation: Advances in theory, measurement, and applied research (pp. 63-81). Washington, DC: American Psychological Association.

Phinney, J. (2006). Ethnic identity exploration in emerging adulthood. In J. Arnett \& J. L. Tanner (Eds.), Coming of age in the 21st century: The lives and contexts of emerging adults (pp. 117-134). Washington, DC: American Psychological Association.

Phinney, J. S., Cantu, C. L., \& Kurtz, D. A. (1997). Ethnic and American identity as predictor of self-esteem among African American, Latino, and While adolescents. Journal of Youth and Adolescents, 26, 165-285.

Phinney, J. S., Horenezyk, G., Liebkind, K., \& Vedder, P. (2001). Ethnic identity, immigration, and well-being: An interactional perspective. Journal of Social Issues, 57, 493-510.

Phinney, J.S., \& Ong, A.D. (2007). Conceptualization and measurement of ethnic identity: Current status and future directions. Journal of Counseling Psychology, $54(3), 271-281$.

Plunkett, J.W. (2008). Plunkett's sport industry almanac 2008: Sport industry market research, statistics, trends \& leading companies. Houston, TX: Plunkett Research.

Pons, F., Laroche, M., Nyeck, S., \& Perreault, S. (2001). Role of sporting events as ethnoculture's emblems: Impact of acculturation and ethnic identity on 
consumers' orientation toward sporting events. Sport Marketing Quarterly, 10, 231-240.

Popp, N. (2007). International student-athlete perception of college sport and its effect on adjustment to college. University of Louisville, $\mathrm{KY}$

Portes, A., \& Zhou, M. (1993). The new second generation: Segmented assimilation and its variants. Annals AAPSS, 530, 74-82.

Redfield, R., Linton, R., \& Herskovits, M. J. (1936). Memorandum for the study of acculturation. American Anthropologist, 38, 149-152.

Rezentes, W. C. (1993). Na Mea Hawaii: A Hawaiian acculturation scale. Psychological Reports, 73, 383-393.

Robinson, M. J., \& Trail, G. T. (2005). Relationships among spectator gender, motives, points of attachment, and sport preference. Journal of Sport Management, 19, $58-80$.

Roberts, R., Phinney, J., Masse, L., Chen, Y., Roberts, C., \& Romero, A. (1999). The structure of ethnic identity in young adolescents from diverse ethnocultural groups. Journal of Early Adolescence, 19, 301-322.

Robertson, R. (1995). Glocalization: Time-space and homogeneity-heterogeneity. In M. Featherstone, S. Lash, \& R. Robertson (Eds.), Global modernities (pp. 25-44). Thousand Oaks, CA: Sage.

Richmond, A. (1993). Reactive migration: Sociological perspectives on refugee movements, Journal of Refugee Studies, 6, 7-24. 
Rivers, D. H., \& DeSchriver, T. D. (2002). Star players, payroll distribution, and Major League Baseball attendance. Sport Marketing Quarterly 11(3), 164-173.

Robinson, M. J., Trail, G. T., \& Kwon, H. (2004). Motives and points of attachment of professional golf spectators. Sport Management Review, 7(2), 167-192.

Rocha, C. (2011). International sport consumer behaviors: The case of the NBA in Brazil. Paper presented at the 2011 North American Society for Sport Management, London, Canada.

Salant, P., \& Dillman, D. A. (1994). How to conduct your own survey. New York: John Wiley \& Sons.

Shackelford, D.E., \& Greenwell, C.T. (2005). Predicting women's Division I sports attendance: An analysis of institutional characteristics. Sport Marketing Quarterly, 14, 139-147.

Sharma, S., Shimp, T. A., \& Shin, J. (1995). Consumer ethnocentrism: A test of antecedents and moderators. Journal of the Academy of Marketing Science, 23(1), 26-37.

Shin, H. K. (2010). The relationship between consumer decision-making styles and acculturation: A study of Korean American golfers in southern California (Doctoral dissertation). Available from ProQuest Dissertations and Theses database. (UMI No.3409358)

Singh, N., Kwon, I., \& Pereira, A. (2003). Cross-cultural consumer socialization: An exploratory study of socialization influences across three ethnic groups. Psychology \& Marketing, 20(10), 867-881. 
Snowde, L. R., \& Hines, A. M. (1999). A scale to assess African American acculturation. Journal of Black Psychology, 25, 36-47.

Social Science Research Council. (1954). Acculturation: An exploratory formulation. American Anthropologist, 56, 973-1000.

Spanberg, E. (2003). Attracting kids to major sports no child's play. Street \& Smith's SportsBusiness Jounral, 6(8), 24.

Stephenson, M. (2000). Development and validation of the Stephenson Multigroup Acculturation Scale (SMAS). Psychological Assessment, 12(1), 77.

Stodolska, M., \& Alexandris, K. (2004). The role of recreational sport in the adaptation of first generation immigrants in the United States. Journal of Leisure Research, $36(3), 379-413$.

Suinn, R. M., Rickard-Figueroa, K., Lew, S., \& Vigil, P. (1987). The Suinn-Lew Asian Self-Identity Acculturation Scale: An initial report. Educational and Psychological Measurement, 47, 401-407.

Sutton, W. A., McDonald, M. A., Milne, G. R., \& Cimperman, J. (1997). Creating and fostering fan identification in professional sports. Sport Marketing Quarterly, $6(1), 15-22$.

Szapocznik, J., \& Kurtinez, W. M. (1980). Acculturation, biculturation and adjustment among Cuban Americans. In A. M. Padilla (Ed.), Acculturation: Theory, models and some new findings (pp. 139-159). Boulder, CO: Westview Press.

Tajfel, H. (1978). Differentiation between social groups. London: Academic Press. Tajfel, H. (1981). Human groups and social categories. New York: Cambridge University Press. 
Tajfel, H. (1982). Social identity and intergroup relations. Cambridge, UK: Cambridge University Press.

Tajfel, H., \& Turner, J. C. (1986). The social identity theory of intergroup behavior. In S. Worchel \& W. Austin (Eds.), Psychology of intergroup relations (2nd ed., pp. 724). Chicago, IL: Nelson-Hall.

Trail, G. T., Anderson, D., \& Fink, J. S. (2000). A theoretical model of sport spectator consumption behavior. International Journal of Sport Management, 12(4), 217227.

Trail, G. T., Anderson, D. F., \& Fink, J. S. (2005). Consumer satisfaction and identity theory: A model of sport spectator conative loyalty. Sport Marketing Quarterly, $14,98-111$.

Trail, G. T., \& James, J. D. (2001). The Motivation Scale for Sport Consumption: Assessment of the scale's psychometric properties. Journal of Sport Behavior, $24,108-127$.

Trail, G. T., Robinson, M. J., Dick, R. J., \& Gillentine, A. J. (2003). Motives and Points of Attachment: Fans Versus Spectators in Intercollegiate Athletics. Sport Marketing Quarterly, 12(4), 217-227.

Triandis, H. C., Bontempo, R., Villareal, M. J., Asai, M., \& Lucca, N. (1988a). Individualism and collectivism: Cross-cultural perspectives on self-ingroup relationships. Journal of Personality and Social Psychology, 54, 323-338.

Tsai, J. L., Ying, Y.W., \& Lee, P. A. (2000). The meaning of 'being Chinese' and 'being American': Variation among Chinese American young adults. Journal of CrossCultural Psychology, 31(3), 302-332. 
U.S. Census Bureau. (2007). The American community-Asians: 2004. American community survey reports. Retrieved from http://www.census.gov/prod/2007pubs/acs-05.pdf

U.S. Census Bureau. (2008). 2005-2007 American community survey 3-year estimates [Data set].Retrieved from http://factfinder.census.gov/home/saff/main.html?_lang=en

U.S. Census Bureau. (2011). Overview of race and Hispanic origin: 2010 Census briefs. Retrieved from http://www.census.gov/prod/cen2010/briefs/c2010br-02.pdf

Venkatesh, A. (1995), Ethnoconsumerism: A new paradigm to study cultural and crosscultural consumer behavior. In I. A. Costa \& G. J. Bamossy (Eds.). Marketing in a multicultural world: Ethnicity, nationalism, and cultural identity (pp. 26-67). Thousand Oaks, CA: Sage Publications.

Wang, R. T., \& Zhang, J. (2009). Studying. Asian Americans' attachment to Asians star athletes in major professional sport leagues: Development of a theoretical framework. Paper presented at the 2009 Sport Marketing Association, Cleveland, $\mathrm{OH}$.

Wann, D. L. (1995). Preliminary validation of the Sport Fan Motivation Scale. Journal of Sport \& Social Issues, 19, 377-396.

Wann, D. L., \& Branscombe, N. R. (1993). Sport fans: Measuring degree of identification with their team. International Journal of Sport Psychology, 24, 117. 
Wann, D. L., \& Branscombe, N. R. (1990). Die-hard and fair-weather fans: Effects of identification on BIRGing and CORFing tendencies. Journal of Sport \& Social Issues, 14, 103-117.

Wann, D. L., \& Branscombe, N.R. (1995). Influence of level of identification with a group and physiological arousal on perceived intergroup complexity. British Journal of Social Psychology, 34, 223-235.

Wann, D. L., \& Dolan, T. J. (1994). Attributions of highly identified sports spectators. Journal of Social Psychology, 134, 783-792.

Wann, D. L., Keean, B. L., Burnett, S., Martin, J., Page, L., \& Smith, L. (2002). The impact of sport team identification and attributions of ability and effort on spectators' impressions of athletic performance. North American Journal of Psychology, 4, 347-354.

Wann, D.L., Schrader, M.P. (1997). Team identification and the enjoyment of watching a sporting event. Perceptual and Motor Skills, 84, 954.

Wann, D.L., Tucker, K.B., \& Schrader, M.P. (1996). An exploratory examination of the factors influencing the origination, continuation, and cessation of identification with sports teams. Perceptual and Motor Skills, 82, 995-1001.

Ward, C. \& Kennedy, A. (1992). Locus of control, mood disturbance and social difficulty during cross-cultural transitions. International Journal of Intercultural Relations, $16,175-194$

Xu, J., Shim, S., Lotz, S., \& Almeida, D. (2004). Ethnic identity, socialization factors, and culture-specific consumption behavior. Psychology \& Marketing, 21, 93112. 
Zhang, J. J., Pease, D. G., Hui, S. C., \& Michaud, T. J. (1995). Variables affecting the spectator decision to attend NBA games. Sport Marketing Quarterly, 4(4), 29-39.

Zhang, J. J., Pease, D. G., Smith, D. W., Lee, J. T., Lam, E. T. C., \& Jambor, E. A. (1997). Factors affecting the decision making of spectators to attend minor league hockey games. International Sports Journal, 1(1), 39-53. 


\title{
APPENDIX A
}

\author{
Email Contact for Data Collection
}

Dear

We are sport administration researchers from the University of Louisville. We are conducting a study regarding sport fan behaviors and would like to collect data from your organization's members.

Our study will investigate how East Asian populations in the U.S. (Chinese, Japanese, Korean, and Taiwanese) consume professional sports. More specifically, we will investigate the impacts of high-profile Asian athletes (e.g., Ichiro Suzuki, Yao Ming, Shin Soo Choo, Hong-Chih Kuo) on Asians' sport consumption behaviors, such as attending games, watching them on TV, and searching for news about the athletes through the Internet.

As a part of the research project, we will collect data from four regions where the Asian athletes play, including Seattle, Houston, Cleveland, and Los Angeles. Your organization is located in one of our target places for data collection, and we would like to collect data using survey questionnaires distributed to your organization's members.

We would like to collect data through one of two options:

1. Paper Survey: The only thing you would need to do is to distribute the survey questionnaires to members in your organization, collect them, and mail back them to us. We will provide paper copies of the surveys and a return envelope with pre-paid postage.

OR

2. Online Survey: The only thing you would need to do is to send an email to members in your organization. The email will include a link to an Internet website on which the survey questionnaire is posted.

Please let us know if we can collect data from your organization this fall. We can be reached through email (mhums@louisville.edu) or phone (502-852-6683).

Thank you in advance for your time and consideration.

Sincerely,

Mary A. Hums, PhD

Professor

Sport Administration

University of Louisville

(502) 852-0555

chris.greenwell@louisville.edu
Jae-Pil Ha

Ph.D. Candidate

Sport Administration

University of Louisville

(551) 795-0418

j0ha0001@louisville.edu 


\section{APPENDIX B \\ The Role of Acculturation and Ethnic Identity in Understanding \\ Asians' Sport Consumption Behavior in the United State}

Date October 2011

Dear Participants:

You are being invited to participate in a research study by answering the attached survey about acculturation, ethnic identity, sport fan identification and consumption behavior among Asians in the U.S. There are no known risks for your participation in this research study. The information collected may not benefit you directly. The information learned in this study may be helpful to others. The information you provide will help sport marketers and researchers better understand Asians' sport consumption attitudes and behaviors. Your completed survey will be stored at the University of Louisville. The survey will take approximately 7 to 10 minutes to complete.

Individuals from the Department of Health and Sport Science, the Institutional Review Board (IRB), the Human Subjects Protection Program Office (HSPPO), and other regulatory agencies may inspect these records. In all other respects, however, the data will be held in confidence to the extent permitted by law. Should the data be published, your identity will not be disclosed.

Taking part in this study is voluntary. By completing this survey you agree to take part in this research study. You do not have to answer any questions that make you uncomfortable. You may choose not to take part at all. If you decide to be in this study you may stop taking part at any time. If you decide not to be in this study or if you stop taking part at any time, you will not lose any benefits for which you may qualify.

If you have any questions, concerns, or complaints about the research study, please contact: Jae-Pil Ha at (551) 795-0418, Dr. Mary A. Hums (502) 852-5908, or Dr. T. Chris Greenwell (502) 852-0555.

If you have any questions about your rights as a research subject, you may call the Human Subjects Protection Program Office at (502) 852-5188. You can discuss any questions about your rights as a research subject, in private, with a member of the Institutional Review Board (IRB). You may also call this number if you have other questions about the research, and you cannot reach the research staff, or want to talk to someone else. The IRB is an independent committee made up of people from the University community, staff of the institutions, as well as people from the community not connected with these institutions. The IRB has reviewed this research study.

If you have concerns or complaints about the research or research staff and you do not wish to give your name, you may call 1-877-852-1167. This is a 24 hour hot line answered by people who do not work at the University of Louisville.

Sincerely,

Jae-Pil Ha Mary A. Hums 


\section{APPENDIX C}

\section{Survey Example for Chinese Sample (English Version)}

\section{Demographic Information}

The following section of the questionnaire is about your background information. Your response will be held in confidence. Please circle the corresponding number for each of the following questions.

1. What is your ethnicity?

1) Chinese (including Chinese American)

2) Japanese (including Japanese American)

3) Korean (including Korean American)

4) Taiwanese (including Taiwanese American)

5) Other (Please specify)

2. What is your gender?

1) Male

2) Female

3. What is your age?

(Please specify)

4. Were you born in the United States? 1) Yes $\quad$ 2) No

If not, how long have you lived in the United States? ___ Years ___Months

5. What is your current marital status?

1) Single, Never married

2) Married

3) Divorced or Separated

4) Widowed

6. What is your highest level of educational achievement?

1) High School

2) Bachelor's Degree

3) Master's Degree

4) Doctoral Degree

5) Other (Please specify) 
7. What is your generation status?

1) Asian: I was born in Asia and live in America temporarily.

2) 1st generation: I was born and grew up in Asia. I live in America as a resident.

3) One and a half generation: I was born in an Asian country. When I was young, I emigrated to America and grew up here.

4) Second generation: My parents are the first generation of immigrants.

5) Third generation: My parents are the second generation of immigrants.

8. What is your current U.S. immigration status?

1) U.S. Citizenship

2) Green Card (Permanent Resident)

3) Student Visa

4) Working Visa

5) Other (specify)

9. What is your annual household income?

1) Less than $\$ 19,999$

2) $\$ 20,000-\$ 39,999$

3) $\$ 40,000-\$ 59,999$

4) $\$ 60,000-\$ 79,999$

5) $\$ 80,000-\$ 99,999$

6) $\$ 100,000+$

10. Who is your favorite Chinese player playing or recently played for an American professional team?

11. What do you think is the most popular sport in China?

12. What do you think is the most popular sport in America? 


\section{Ethnic Identity Scale for Chinese}

The following 3 items are concerned with how you feel about your ethnic group (Chinese). The items are in the form of rating scales, ranging from the lowest score of 1 $=$ Strongly Disagree to the highest score of $7=$ Strongly Agree. Please indicate how much you agree or disagree with each statement by circling the appropriate number. Be sure that your ethnic group is Chinese.

\begin{tabular}{|c|c|c|c|c|c|c|c|c|}
\hline \multirow[b]{2}{*}{1.} & \multirow[b]{2}{*}{$\begin{array}{l}\text { I have a strong sense of belonging to my own } \\
\text { ethnic group (Chinese). }\end{array}$} & \multicolumn{3}{|c|}{$\begin{array}{l}\text { Strongly } \\
\text { Disagree }\end{array}$} & & \multicolumn{3}{|c|}{$\begin{array}{l}\text { Strongly } \\
\text { Agree }\end{array}$} \\
\hline & & 1 & 2 & 3 & 4 & 5 & 6 & 7 \\
\hline 2. & $\begin{array}{l}\text { I feel a strong attachment to my own ethnic group } \\
\text { (Chinese). }\end{array}$ & 1 & 2 & 3 & 4 & 5 & 6 & 7 \\
\hline 3. & $\begin{array}{l}\text { I understand pretty well what my ethnic group } \\
\text { membership (Chinese) means to me. }\end{array}$ & 1 & 2 & 3 & 4 & 5 & 6 & 7 \\
\hline
\end{tabular}

\section{Acculturation Scale for Chinese}

The following questions are concerned with how you feel and behave with your culture of origin (China) and with American culture. Please answer the questions below by circling a number from 1 (Not at All) to 7 (Very Much). Be sure that your culture of origin is China.

* Culture of Origin $=$ China

\begin{tabular}{|c|c|c|c|c|c|c|c|c|}
\hline \multirow[b]{2}{*}{1.} & \multirow[b]{2}{*}{$\begin{array}{l}\text { How much do you feel you have in common } \\
\text { with people from China? }\end{array}$} & \multicolumn{3}{|c|}{ Not at All } & \multicolumn{4}{|c|}{ Very Much } \\
\hline & & 1 & 2 & 3 & 4 & 5 & 6 & 7 \\
\hline 2. & $\begin{array}{l}\text { How much do you interact and associate with } \\
\text { people from China? }\end{array}$ & 1 & 2 & 3 & 4 & 5 & 6 & 7 \\
\hline 3. & $\begin{array}{l}\text { How much do you identify with people from } \\
\text { China? }\end{array}$ & 1 & 2 & 3 & 4 & 5 & 6 & 7 \\
\hline 4. & $\begin{array}{l}\text { How much would you like to interact and } \\
\text { associate with people from China? }\end{array}$ & 1 & 2 & 3 & 4 & 5 & 6 & 7 \\
\hline 5. & How proud are you to be a part of China? & 1 & 2 & 3 & 4 & 5 & 6 & 7 \\
\hline 6. & $\begin{array}{l}\text { How negative do you feel about people of } \\
\text { China? }\end{array}$ & 1 & 2 & 3 & 4 & 5 & 6 & 7 \\
\hline 7. & How well do you speak the language of Chinese? & 1 & 2 & 3 & 4 & 5 & 6 & 7 \\
\hline 8. & How well do you understand the language of & & & & & & & \\
\hline
\end{tabular}




\begin{tabular}{|c|c|c|c|c|c|c|c|c|}
\hline & Chinese? & 1 & 2 & 3 & 4 & 5 & 6 & 7 \\
\hline 9. & $\begin{array}{l}\text { How well do you read and write in the language } \\
\text { of Chinese? }\end{array}$ & 1 & 2 & 3 & 4 & 5 & 6 & 7 \\
\hline 10. & $\begin{array}{l}\text { How often do you listen to music or look at } \\
\text { movies and magazines from China? }\end{array}$ & 1 & 2 & 3 & 4 & 5 & 6 & 7 \\
\hline 11. & $\begin{array}{l}\text { How knowledgeable are you about the culture } \\
\text { and traditions of China? }\end{array}$ & 1 & 2 & 3 & 4 & 5 & 6 & 7 \\
\hline 12. & $\begin{array}{l}\text { How knowledgeable are you about the history of } \\
\text { China? }\end{array}$ & 1 & 2 & 3 & 4 & 5 & 6 & 7 \\
\hline 13. & $\begin{array}{l}\text { How much do you actually practice the traditions } \\
\text { and keep the holidays of China? }\end{array}$ & 1 & 2 & 3 & 4 & 5 & 6 & 7 \\
\hline 14. & How often do you actually eat Chinese food? & 1 & 2 & 3 & 4 & 5 & 6 & 7 \\
\hline 15. & How much do you like Chinese food? & 1 & 2 & 3 & 4 & 5 & 6 & 7 \\
\hline 16. & $\begin{array}{l}\text { How much do you feel you have in common } \\
\text { with people from American culture? }\end{array}$ & 1 & 2 & 3 & 4 & 5 & 6 & 7 \\
\hline 17. & $\begin{array}{l}\text { How much do you interact and associate with } \\
\text { people from American culture? }\end{array}$ & 1 & 2 & 3 & 4 & 5 & 6 & 7 \\
\hline 18. & $\begin{array}{l}\text { How much do you identify with people from } \\
\text { American culture? }\end{array}$ & 1 & 2 & 3 & 4 & 5 & 6 & 7 \\
\hline 19. & $\begin{array}{l}\text { How much would you like to interact and } \\
\text { associate with people from American culture? }\end{array}$ & 1 & 2 & 3 & 4 & 5 & 6 & 7 \\
\hline 20. & $\begin{array}{l}\text { How proud are you to be a part of American } \\
\text { culture? }\end{array}$ & 1 & 2 & 3 & 4 & 5 & 6 & 7 \\
\hline 21. & $\begin{array}{l}\text { How negative do you feel about people from } \\
\text { American culture? }\end{array}$ & 1 & 2 & 3 & 4 & 5 & 6 & 7 \\
\hline 22. & How well do you speak American English? & 1 & 2 & 3 & 4 & 5 & 6 & 7 \\
\hline 23. & How well do you understand American English? & 1 & 2 & 3 & 4 & 5 & 6 & 7 \\
\hline 24. & $\begin{array}{l}\text { How well do you read and write in American } \\
\text { English? }\end{array}$ & 1 & 2 & 3 & 4 & 5 & 6 & 7 \\
\hline 25. & $\begin{array}{l}\text { How often do you listen to music or look at } \\
\text { movies and magazines from American culture? }\end{array}$ & 1 & 2 & 3 & 4 & 5 & 6 & 7 \\
\hline 26. & $\begin{array}{l}\text { How knowledgeable are you about the culture } \\
\text { and traditions of American culture? }\end{array}$ & 1 & 2 & 3 & 4 & 5 & 6 & 7 \\
\hline 27. & $\begin{array}{l}\text { How knowledgeable are you about the history of } \\
\text { American culture? }\end{array}$ & 1 & 2 & 3 & 4 & 5 & 6 & 7 \\
\hline 28. & $\begin{array}{l}\text { How much do you practice the traditions and } \\
\text { keep the holidays of American culture? }\end{array}$ & 1 & 2 & 3 & 4 & 5 & 6 & 7 \\
\hline 29. & How often do you eat American food? & 1 & 2 & 3 & 4 & 5 & 6 & 7 \\
\hline 30. & $\begin{array}{l}\text { How much do you like the food of American } \\
\text { culture? }\end{array}$ & 1 & 2 & 3 & 4 & 5 & 6 & 7 \\
\hline
\end{tabular}




\section{Fan Identification and Sport Consumption Behaviors for Chinese}

A. Instructions: The following eight items are concerned with your identification with and consumption behavior for Chinese athletes (e.g., Yao Ming, Yi Jianlian) plaving or recently played on American professional teams. Please indicate how much you agree or disagree with each statement by circling the appropriate number using a 7-point scale, ranging from the lowest score of $1=$ Strongly Disagree to the highest score of $7=$ Strongly Agree.

\begin{tabular}{|c|c|c|c|c|c|c|c|c|}
\hline \multirow[b]{2}{*}{1.} & \multirow[b]{2}{*}{$\begin{array}{l}\text { I identify with a specific player from China } \\
\text { playing on an American team. }\end{array}$} & \multicolumn{3}{|c|}{$\begin{array}{l}\text { Strongly } \\
\text { Disagree }\end{array}$} & \multicolumn{4}{|c|}{$\begin{array}{l}\text { Strongly } \\
\text { Agree }\end{array}$} \\
\hline & & 1 & 2 & 3 & 4 & 5 & 6 & 7 \\
\hline 2. & $\begin{array}{l}\text { I am a big fan of a specific player from China } \\
\text { playing on an American team. }\end{array}$ & 1 & 2 & 3 & 4 & 5 & 6 & 7 \\
\hline 3. & $\begin{array}{l}\text { I consider myself a fan of specific player from } \\
\text { China playing on an American team. }\end{array}$ & 1 & 2 & 3 & 4 & 5 & 6 & 7 \\
\hline 4. & $\begin{array}{l}\text { I am more likely to attend the game(s) of an } \\
\text { American team having a player from China. }\end{array}$ & 1 & 2 & 3 & 4 & 5 & 6 & 7 \\
\hline 5 & $\begin{array}{l}\text { I watch or listen to the game(s) of an American } \\
\text { team with a player from China through the media } \\
\text { (e.g., TV, Internet, Radio, etc.). }\end{array}$ & 1 & 2 & 3 & 4 & 5 & 6 & 7 \\
\hline 6. & $\begin{array}{l}\text { I follow the news on an American team with a } \\
\text { player from China through the media (e.g., TV, } \\
\text { Internet, Radio, etc.). }\end{array}$ & 1 & 2 & 3 & 4 & 5 & 6 & 7 \\
\hline 7. & $\begin{array}{l}\text { I purchase the team's merchandise from an } \\
\text { American team that has a player from China. }\end{array}$ & 1 & 2 & 3 & 4 & 5 & 6 & \\
\hline 8. & $\begin{array}{l}\text { I buy the team's clothing from an American team } \\
\text { that has a player from China. }\end{array}$ & 1 & 2 & 3 & 4 & 5 & 6 & \\
\hline
\end{tabular}

B. Instructions: The following seven items are concerned with your identification with and consumption behavior for a popular sport in China (e.g., basketball). Please indicate how much you agree or disagree with each statement by circling the appropriate number using a 7-point scale, ranging from the lowest score of $1=$ Strongly Disagree to the highest score of $7=$ Strongly Agree .

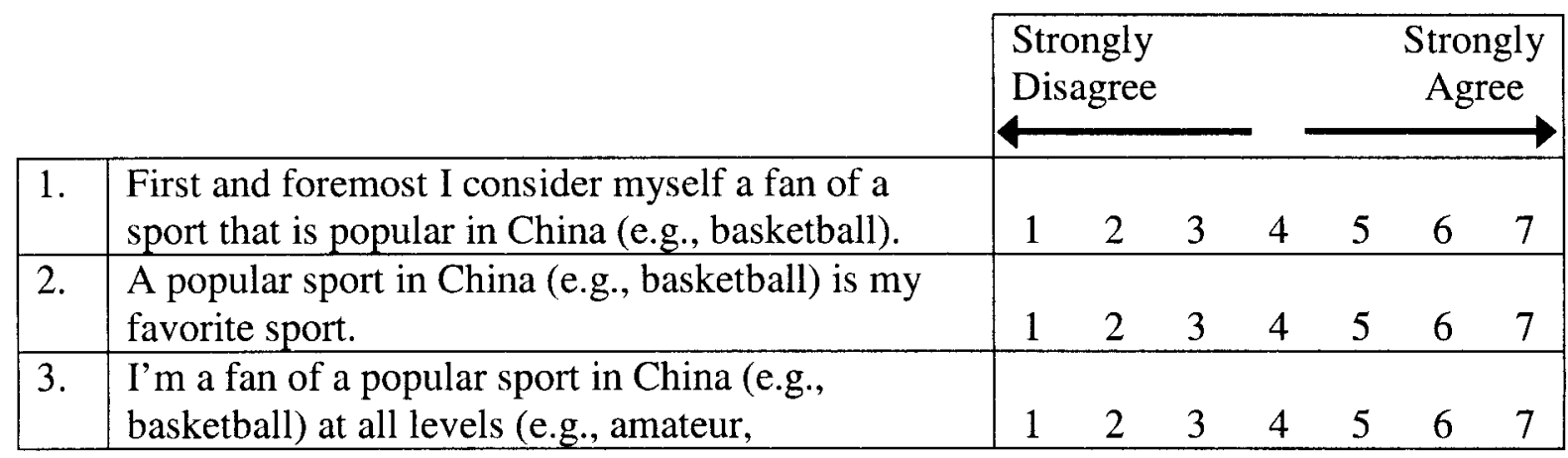




\begin{tabular}{|l|l|rrrrrrr|}
\hline & professional). & & & & & & \\
\hline 4. & $\begin{array}{l}\text { I watch or listen to a popular sport in China (e.g., } \\
\text { basketball) through the Internet. }\end{array}$ & 1 & 2 & 3 & 4 & 5 & 6 & 7 \\
\hline 5 & $\begin{array}{l}\text { I follow the news on a popular sport in China (e.g., } \\
\text { basketball) through the Internet. }\end{array}$ & 1 & 2 & 3 & 4 & 5 & 6 & 7 \\
\hline 6 & $\begin{array}{l}\text { I purchase the merchandise of a popular sport in } \\
\text { China (e.g., basketball). }\end{array}$ & 1 & 2 & 3 & 4 & 5 & 6 & 7 \\
\hline 7. & $\begin{array}{l}\text { I buy the clothing of a popular sport in China (e.g., } \\
\text { basketball). }\end{array}$ & 1 & 2 & 3 & 4 & 5 & 6 & 7 \\
\hline
\end{tabular}

C. Instructions: The following eight items are concerned with your identification with and consumption behavior for a popular sport in America (e.g., American football). Please indicate how much you agree or disagree with each statement by circling the appropriate number using a 7-point scale, ranging from the lowest score of $1=$ Strongly Disagree to the highest score of $7=$ Strongly Agree.

\begin{tabular}{|c|c|c|c|c|c|c|c|c|}
\hline & & & & & & & & \\
\hline 1. & $\begin{array}{l}\text { First and foremost I consider myself a fan of a } \\
\text { popular sport in America (e.g., football). }\end{array}$ & 1 & 2 & 3 & 4 & 5 & 6 & 7 \\
\hline 2. & $\begin{array}{l}\text { A popular sport in America (e.g., football) is my } \\
\text { favorite sport. }\end{array}$ & 1 & 2 & 3 & 4 & 5 & 6 & 7 \\
\hline 3. & $\begin{array}{l}\text { I'm a fan of a popular sport in America (e.g., } \\
\text { football) at all levels (e.g., amateur, professional) }\end{array}$ & 1 & 2 & 3 & 4 & 5 & 6 & 7 \\
\hline 4. & $\begin{array}{l}\text { I attend a popular sport game(s) in America (e.g., } \\
\text { football). }\end{array}$ & 1 & 2 & 3 & 4 & 5 & 6 & 7 \\
\hline 5 & $\begin{array}{l}\text { I watch or listen to a popular sport game(s) in } \\
\text { America (e.g., football) through the media (e.g., } \\
\text { TV, Internet, Radio, etc.). }\end{array}$ & 1 & 2 & 3 & 4 & 5 & 6 & 7 \\
\hline 6. & $\begin{array}{l}\text { I follow the news on a popular sport in America } \\
\text { (e.g., football) through the media (e.g., TV, } \\
\text { Internet, Radio, etc.). }\end{array}$ & 1 & 2 & 3 & 4 & 5 & 6 & 7 \\
\hline 7. & $\begin{array}{l}\text { I purchase the merchandise of a popular sport in } \\
\text { America (e.g., football). }\end{array}$ & 1 & 2 & 3 & 4 & 5 & 6 & 7 \\
\hline 8. & $\begin{array}{l}\text { I buy the clothing of a popular sport in America } \\
\text { (e.g., football). }\end{array}$ & 1 & 2 & 3 & 4 & 5 & 6 & \\
\hline
\end{tabular}




\section{Survey Example for Chinese Sample (Chinese Version) \\ 中国受试者问卷 \\ 基本资料}

以下问题为您的个人基本资料. 您的资料将会保密. 请圈选下列每一个问题前方的 数字.

1.请问您是什么种族?

1) 中国人(包含华裔美国人)

2) 日本人 (包含华裔美国人)

3）韩国人 (包含韩裔美国人)

4) 台湾人 (包含台裔美国人)

5) 其他 (请说明)

2. 请问您的性别?

1) 男

2) 女

3. 请问你的年龄?

(请明确说明)

4. 请问您在美国出生吗?

1) 是

2) 否

如不是, 您已经在美国住了多久? 年 个月

5. 请问您目前的婚姻状况?
1) 单身, 从未结婚
2) 已婚
3) 离婚或分居
4) 伴侣已过世

6. 请问您的最高学历
1) 高中
2) 大专
3) 硕士
4) 博士
5) 其他 (请明确说明)

7. 请问您属于哪种世代状态

1) 亚洲人: 出生于亚洲, 暂时住在美国

2) 第一代: 出生并成长于亚洲. 现为美国合法居民

3) 第 1.5 代: 出生于其他国家, 年轻时移民美国并在此成长.

4) 第二代: 父母是第一代移民

5) 第三代: 父母是第二代移民 
8. 请问您目前居留美国的身分

1) 美国公民

2) 绿卡 (永久居民)

3) 学生签证

4) 工作签证

5) 其他 (请说明)

9. 请问您的年家庭收入

1) 少于 $\$ 19,999$

2) $\$ 20,000-\$ 39,999$

3) $\$ 40,000-\$ 59,999$

4) $\$ 60,000-\$ 79,999$

5) $\$ 80,000-\$ 99,999$

6) $\$ 100,000+$

10. 请问美国职业球队中. 谁是您最欣赏的现役或最近的中国球员?

11.您觉得中国最流行的运动是？

12.您觉得美国最流行的运动是?

\section{中国人种族认同量表}

下列三个问题是关于您对种族团体(中国)的意见. 问题以量表方式, 从最低分数 $1=$ 非常不同意至 $7=$ 非常同意. 请指出你对每项说明的认同或不认同程度 并圈选最 最适当的数字. 请确认您的种族为中国人.

\begin{tabular}{|c|c|c|c|c|c|c|c|c|}
\hline \multirow[b]{2}{*}{1.} & & \multicolumn{3}{|c|}{ 非常不同意 } & \multicolumn{4}{|c|}{ 非常同意 } \\
\hline & $\begin{array}{l}\text { 我对身为我的种族团体(中国人)有强烈的认同 } \\
\text { 感 }\end{array}$ & 1 & 2 & 3 & 4 & 5 & 6 & 7 \\
\hline 2. & $\begin{array}{l}\text { 我感觉到我对我自身的的种族团体(中国人)有 } \\
\text { 强烈的连结与依附感 }\end{array}$ & 1 & 2 & 3 & 4 & 5 & 6 & 7 \\
\hline 3. & 我十分了解我的种族团体(中国人)对我的意义 & 1 & 2 & 3 & 4 & 5 & 6 & 7 \\
\hline
\end{tabular}




\section{中国人文化适应量表}

下列问题是关于您对您原本的(中国)文化与美国文化之间的感受及行为. 请圈选 1 (完全没有)-7(非常多)

* 原本文化 $=$ 中国

\begin{tabular}{|c|c|c|c|c|c|c|c|c|}
\hline & & & 殳 & & & & & \\
\hline 1. & 您觉得您与来自中国的人有多少共同点? & 1 & 2 & 3 & 4 & 5 & 6 & 7 \\
\hline 2. & 您与来自中国的人互动及连结有多少? & 1 & 2 & 3 & 4 & 5 & 6 & 7 \\
\hline 3. & 您与来自中国的人打成一片的程度有多少? & 1 & 2 & 3 & 4 & 5 & 6 & 7 \\
\hline 4. & $\begin{array}{l}\text { 您对与来自中国的人的互动及连结的意愿有多 } \\
\text { 少? }\end{array}$ & 1 & 2 & 3 & 4 & 5 & 6 & 7 \\
\hline 5 . & 你对身为中国人感到骄傲的程度有多少? & 1 & 2 & 3 & 4 & 5 & 6 & 7 \\
\hline 6. & 您对中国人负面的感觉有多少? & 1 & 2 & 3 & 4 & 5 & 6 & 7 \\
\hline 7. & 您说中文的程度如何? & 1 & 2 & 3 & 4 & 5 & 6 & 7 \\
\hline 8. & 您了解中文的程度如何? & 1 & 2 & 3 & 4 & 5 & 6 & 7 \\
\hline 9. & 您读及写中文的程度如何？ & 1 & 2 & 3 & 4 & 5 & 6 & 7 \\
\hline 10. & 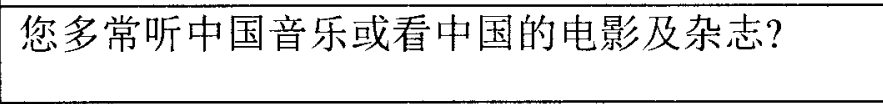 & 1 & 2 & 3 & 4 & 5 & 6 & 7 \\
\hline 11. & 您对中国文化及传统的知识有多少? & 1 & 2 & 3 & 4 & 5 & 6 & 7 \\
\hline 12 . & 您对中国历史的知识有多少? & 1 & 2 & 3 & 4 & 5 & 6 & 7 \\
\hline 13. & $\begin{array}{l}\text { 您对中国传统的实践及过中国的节日程度有多 } \\
\text { 少? }\end{array}$ & 1 & 2 & 3 & 4 & 5 & 6 & 7 \\
\hline 14. & 您多常吃中国食物？ & 1 & 2 & 3 & 4 & 5 & 6 & 7 \\
\hline 15. & 您有多喜爱中国食物? & 1 & 2 & 3 & 4 & 5 & 6 & 7 \\
\hline 16. & 您觉得您与来自美国文化的人有多少共同点? & 1 & 2 & 3 & 4 & 5 & 6 & 7 \\
\hline 17. & 您与来自美国文化的人互动及连结有多少? & 1 & 2 & 3 & 4 & 5 & 6 & 7 \\
\hline 18. & 您与来自美国文化的人打成一片的程度有多少? & 1 & 2 & 3 & 4 & 5 & 6 & 7 \\
\hline
\end{tabular}




\begin{tabular}{|c|c|c|c|c|c|c|c|c|}
\hline 19. & $\begin{array}{l}\text { 您对与来自美国文化的人互动及连结的意愿有 } \\
\text { 多少? }\end{array}$ & 1 & 2 & 3 & 4 & 5 & 6 & 7 \\
\hline 20. & $\begin{array}{l}\text { 你对身为美国文化一部分感到骄傲的程度有多 } \\
\text { 少？ }\end{array}$ & 1 & 2 & 3 & 4 & 5 & 6 & 7 \\
\hline 21. & 您对来自美国文化的人负面的感觉有多少? & 1 & 2 & 3 & 4 & 5 & 6 & 7 \\
\hline 22. & 您说英文的程度如何? & 1 & 2 & 3 & 4 & 5 & 6 & 7 \\
\hline 23. & 您对英文的了解程度如何? & 1 & 2 & 3 & 4 & 5 & 6 & 7 \\
\hline 24. & 您读及写英文的程度如何? & 1 & 2 & 3 & 4 & 5 & 6 & 7 \\
\hline 25. & 您多常听美国音乐或看美国的电影及杂志? & 1 & 2 & 3 & 4 & 5 & 6 & 7 \\
\hline 26. & 您对美国文化及传统的知识有多少? & 1 & 2 & 3 & 4 & 5 & 6 & 7 \\
\hline 27. & 您对美国历史的知识有多少?? & 1 & 2 & 3 & 4 & 5 & 6 & 7 \\
\hline 28. & $\begin{array}{l}\text { 您对美国传统的实践及过美国的节日程度有多 } \\
\text { 少? }\end{array}$ & 1 & 2 & 3 & 4 & 5 & 6 & 7 \\
\hline 29. & 您多常吃美国食物? & 1 & 2 & 3 & 4 & 5 & 6 & 7 \\
\hline 30. & 您有多喜爱美国食物? & 1 & 2 & 3 & 4 & 5 & 6 & 7 \\
\hline
\end{tabular}

\section{中国人球迷认同 与运动消费行为}

A. 说明: 下列八个问题是关于您对在美国职业球队打球的现役或最近中国运动 员(例如 姚明，易建联)之认同感及消费行为. 请圈选适当之数字表示您对每项 说明的同意程度. 此为七分量表从最低分数 $1=$ 非常不同意至最高分数 $7=$ 非 常同意

\begin{tabular}{|c|c|c|c|c|c|c|c|c|}
\hline \multirow[b]{2}{*}{1 . } & \multirow[b]{2}{*}{ 我对一特定在美国球队打球的中国球员有认同 } & \multicolumn{3}{|c|}{ 非常不同意 } & & \multicolumn{3}{|c|}{ 非常同意 } \\
\hline & & 1 & 2 & 3 & 4 & 5 & 6 & 7 \\
\hline 2. & $\begin{array}{l}\text { 我是一特定在美国球队打球之中国球员的热衷 } \\
\text { 球迷 }\end{array}$ & 1 & 2 & 3 & 4 & 5 & 6 & 7 \\
\hline 3. & $\begin{array}{l}\text { 我认为我自己是一个特定在美国球队打球的中 } \\
\text { 国球员的球迷 }\end{array}$ & 1 & 2 & 3 & 4 & 5 & 6 & 7 \\
\hline 4. & 我比较愿意去现场看有中国球员的美国球队比 & 1 & 2 & 3 & 4 & 5 & 6 & 7 \\
\hline
\end{tabular}




\begin{tabular}{|c|c|c|c|c|c|c|c|c|}
\hline & 赛 & & & & & & & \\
\hline 5 & $\begin{array}{l}\text { 我透过媒体(电视, 网络, 广播)收看或收听有中 } \\
\text { 国球员的美国球队比赛 }\end{array}$ & 1 & 2 & 3 & 4 & 5 & 6 & 7 \\
\hline 6. & $\begin{array}{l}\text { 我透过媒体(电视, 网络, 广播)密切注意有中国 } \\
\text { 球员的美国球队比赛 }\end{array}$ & 1 & 2 & 3 & 4 & 5 & 6 & 7 \\
\hline 7. & 我购买有中国球员的美国球队相关产品 & 1 & 2 & 3 & 4 & 5 & 6 & 7 \\
\hline 8. & 我购买有中国球员的美国球队衣服 & 1 & 2 & 3 & 4 & 5 & 6 & 7 \\
\hline
\end{tabular}

B. 说明: 下列七个问题是关于您对中国热门的运动(例如 篮球)之认同感及消费 行为. 请圈选适当之数字表示您对每项说明的同意程度. 此为七分量表从最低分 数 $1=$ 非常不同意至最高分数 $7=$ 非常同意

\begin{tabular}{|c|c|c|c|c|c|c|c|c|}
\hline \multirow[b]{2}{*}{1.} & \multirow[b]{2}{*}{$\begin{array}{l}\text { 我认为我自己是一个中国热门运动(例如: 篮球) } \\
\text { 的球迷是最重要的 }\end{array}$} & \multicolumn{3}{|c|}{ 非常不同意 } & \multicolumn{4}{|c|}{ 非常同意 } \\
\hline & & 1 & 2 & 3 & 4 & 5 & 6 & 7 \\
\hline 2 . & $\begin{array}{l}\text { 中国最热门的运动(例如:篮球)是我最喜欢的运 } \\
\text { 动 }\end{array}$ & 1 & 2 & 3 & 4 & 5 & 6 & 7 \\
\hline 3. & $\begin{array}{l}\text { 我是中国热门运动(例如: 篮球)各个级别(例如: } \\
\text { 业余，职业)的球迷. }\end{array}$ & 1 & 2 & 3 & 4 & 5 & 6 & 7 \\
\hline 4. & $\begin{array}{l}\text { 我利用网络收看或收听中国的热门运动 (例如: } \\
\text { 篮球) }\end{array}$ & 1 & 2 & 3 & 4 & 5 & 6 & 7 \\
\hline 5 & $\begin{array}{l}\text { 我利用网络密切注意中国的热门运动 (例如:篮 } \\
\text { 球)的新闻 }\end{array}$ & 1 & 2 & 3 & 4 & 5 & 6 & 7 \\
\hline 6 & 我购买中国热门运动 (例如:篮球)的相关产品 & 1 & 2 & 3 & 4 & 5 & 6 & 7 \\
\hline 7. & 我购买中国热门运动 (例如:篮球)的衣服. & 1 & 2 & 3 & 4 & 5 & 6 & 7 \\
\hline
\end{tabular}

C. 说明: 下列八个问题是关于您对在美国热门运动(例如: 美式足球)之认同感及 消费行为. 请圈选适当之数字表示您对每项说明的同意程度. 此为七分量表从最 低分数 $1=$ 非常不同意至最高分数 $7=$ 非常同意

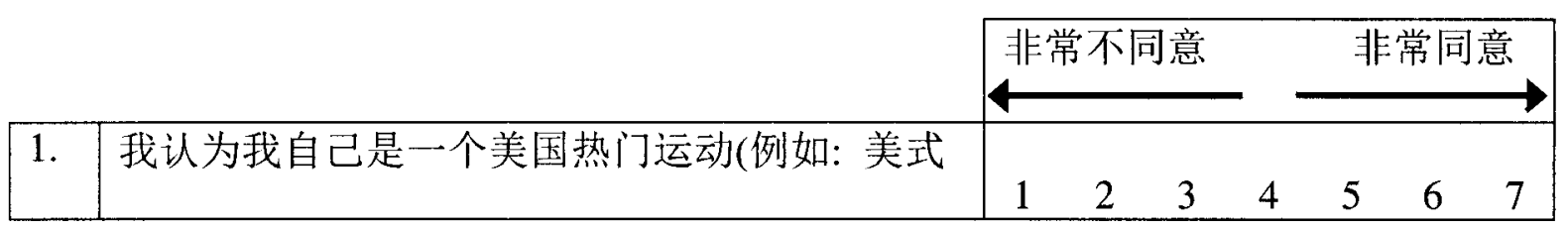




\begin{tabular}{|c|c|c|c|c|c|c|c|c|}
\hline & 足球)的球迷是最重要的 & & & & & & & \\
\hline 2. & $\begin{array}{l}\text { 美国热门运动(例如: 美式足球)是我最喜欢的运 } \\
\text { 动 }\end{array}$ & 1 & 2 & 3 & 4 & 5 & 6 & 7 \\
\hline 3. & $\begin{array}{l}\text { 我是美国热门运动(例如：美式足球)各个级别 } \\
\text { (例如:业余，职业)的球迷. }\end{array}$ & 1 & 2 & 3 & 4 & 5 & 6 & 7 \\
\hline 4. & 我会去看美国热门运动(例如：美式足球)的球赛 & 1 & 2 & 3 & 4 & 5 & 6 & 7 \\
\hline 5 & $\begin{array}{l}\text { 我利用媒体(例如: 电视, 网络, 广播)收看或收 } \\
\text { 听美国热门运动(例如: 美式足球) }\end{array}$ & 1 & 2 & 3 & 4 & 5 & 6 & 7 \\
\hline 6. & $\begin{array}{l}\text { 我利用媒体(例如: 电视, 网络, 广播)密切注意 } \\
\text { 美国热门运动(例如: 美式足球)的新闻 }\end{array}$ & 1 & 2 & 3 & 4 & 5 & 6 & 7 \\
\hline 7. & $\begin{array}{l}\text { 我购买美国热门运动(例如：美式足球)的相关产 } \\
\text { 品 }\end{array}$ & 1 & 2 & 3 & 4 & 5 & 6 & 7 \\
\hline 8. & 我购买美国热门运动(例如: 美式足球)的衣服. & 1 & 2 & 3 & 4 & 5 & 6 & 7 \\
\hline
\end{tabular}




\section{APPENDIX D}

\section{Sport Consumption Behavior in the U.S.}

\section{Comment Form}

Please read the survey and respond to the following statements in the space provided. Feel free to write directly on the questionnaire. Any suggestions and comments for improvement of the survey will be appreciated.

The purposes of this survey are to: (a) assess levels of ethnic identity and acculturation; (b) assess levels of identification with and consumption behavior for Asian athletes playing on American profession teams, a popular sport in a native country (e.g., China, Japan, Korea, and Taiwan), a popular sport in a host country (U.S.), respectively; and (c) collect demographic information.

1) Given the purpose of this study, do you think the questions/items on the survey collect the information needed? Why or why not?

2) In the phrasing and terminology clear and easy to understand?

3) Are the directions easy to follow? 
(a) Is the survey too long to be comfortably completed in one sitting?

(b) Approximately how long would it take you to complete it?

5) Is there any important background information that may be missing?

6) Are there any statements or categories that should be added or deleted? If so, please explain.

7) Please include any other comments relevant to the improvement of this survey 


\section{CURRICULUM VITA JAE-PIL HA}

Work Address

Department of Health and Sport Sciences

University of Louisville

HP/Theater Arts Room 110B

Louisville, KY 40292

Office: 502-852-0312

Email: j0ha0001@ @ louisville.edu
Home Address

1709 West Creek Way \#4

Louisville, KY 40242

Phone: (551) 795 - 0418

E-mail: physicalhjp@gmail.com

\section{HIGHER EDUCATION}

UNIVERSITY OF LOUISVILLE

Louisville, KY

$\mathrm{Ph} . \mathrm{D}$. in Educational Leadership and

May, 2012

Organizational Development

Concentration: Sport Marketing

Dissertation: "The Role of Acculturation and Ethnic Identity in Understanding Asians' Sport Consumption Behavior in the United States"

Co-advisors: Dr. Mary A. Hums and Dr. Chris Greenwell

UNIVERSITY OF LOUISVILLE

M.S. in Sport Administration

GYEONGSANG NATIONAL

UNIVERSITY

B.A. in Physical Education
Louisville, KY

December, 2008

Jinju, South Korea

February, 2003

\section{PROFESSIONAL EXPERIENCE}


Assistant Professor, August 2012

Department of Health Sciences, University of Arkansas at Little Rock

Graduate Teaching Assistant, August 2011 - May 2012

Department of Health and Sport Sciences, University of Louisville

\section{Courses Taught:}

- Research Methods in Health and Sport Sciences: HSS-604 (Fall 2011, Spring 2012) - This class is designed to introduce quantitative and qualitative research techniques utilized in health and sport sciences, with an emphasis on practical application for master's students. Course topics include: understanding the research process, research design, survey designs, sampling procedures, literature review, identifying the research problem, variables and hypotheses/research questions, reliability and validity, measurement, and basic data analysis.

- Prepared course syllabi, class lectures, Power Point presentations, class assignments, created and maintained Blackboard modules for class

- Advised master's students for their individual research projects

\section{- Sport Marketing: SPAD-383 (Spring 2012)}

This course introduces students to the application of basic principles of marketing to the managed sport industry with emphasis on intercollegiate athletics, professional sport and multisport club operations.

- Prepared course syllabi, class lectures, Power Point presentations, class assignments, created and maintained Blackboard modules for class

Graduate Research Assistant, August 2009 - December 2011

Department of Health and Sport Sciences, University of Louisville

- Assisted in writing research manuscripts and grants proposal 
- Performed literature searches and reviews

- Assisted with data collection and analysis

- Assisted with Institutional Review Board (Human Subjects) applications

- Maintained grade records and Blackboard class module

- Assisted to the Editor, Sport Management Education Journal

Sport Information Intern, May 2008 - Dec. 2008

Athletic Department, University of Louisville

- Posted rosters, stats and bios online for Softball and Men's Tennis

- Uploaded game summaries and video-recorded games on the Internet

- Assisted in developing media guides for Women's Basketball and Men's Tennis

\section{P.E. Teacher and Head Swimming Coach,}

Jin-Gyo Middle School, Gyeong-Nam, Korea, Mar. 2007 - Aug. 2007

- Student-athletes won 13 gold medals in individual competition at 2007 State Youth Sports Festival.

- Student-athletes won a bronze medal from individual competition (50m breaststroke) at Korea National Youth Sports Festival

- Taught "rope-jumping with music" as part of after-school program for 7 th to 9 th grade students.

P.E. Teacher and Head Ssireum Coach (Korean Traditional Wrestling)

Jin-Hae Middle School, Gyeong-Nam, Korea, Sept. 2005 - Feb. 2007

- Classroom Teacher for the 8th and 9th grade

- Taught Physical Education for the 8th and 9th grade

- Developed and implemented lesson plans

\section{P.E. Teacher and Basketball Coach}

Jin-Hae Middle School, Gyeong-Nam, Korea, Mar. 2003 - Aug. 2003 
- Head Coach of men's basketball team and Classroom Teacher for 7th grade

- Won a first prize at 2003 Gyeong-Nam Club Basketball Contest.

- Participated in the project to revitalize School Physical Education (acquired prize money of approximately $\$ 8,000$ )

\section{REFERRED PUBLICATIONS}

1. Ha, J. P., \& Park, S. H. (in press). Understanding of newcomers' acculturation and ethnic identity through sport. Accepted by Korean Journal of Sport Science [Korean Version]

2. Han, K., Ha, J. P., \& Ha, J. (in press). The influence of marketing mix factor of professional baseball franchise on spectator satisfaction, team loyalty, and intention to attend games. Accepted by Journal of Sport and Leisure Studies.

3. Han, D., \& Ha, J. P. (2011). Relationships between fanship and cultural value orientation based on significant others. Korean Society of Sports Science, 20(5), 183-195. [Korean Version].

4. Ha, J. P., King, K. M., \& Naeger, D. J. (2011). The impact of burnout on work outcomes among Korean physical education teachers. Journal of Sport Behavior, 34 (4), 343-357.

5. Ha, J. P., Hums, M. A., \& Greenwell, T. C. (2011). The dual role of physical education teacher-athletic directors in the Korean secondary school. The Physical Educator, 68 (4), 221-233.

6. Park, S. H., Ha, J. P., \& Han, S. J. (2009). A discourse on the future study and policy development of the Korean school elite sports system. Korean Society of Sports Science, 18(2), 585-595. [Korean Version]

\section{MANUSCRIPTS IN REVIEW}

1. Della, L. J., King, K. M., \& Ha, J. P. (in review). The psychometrical properties of perceived susceptibility and perceived severity scales in a population at risk for Type 2 diabetes. Paper submitted to Journal of Rural Health. 
2. Ha, J. P., Hums, M. A., \& Greenwell, T. C. (in review). The impact of acculturation strategies on American football identification and consumption among Asians in the United States. Paper submitted to International Journal of Sports Marketing and Sponsorship.

3. Hancock, M., Lyras, A., \& Ha, J. P. (in review). Global assessment of sport for development program for girls and women. Paper submitted to Journal of Sport for Development.

4. Ha, J. P., \& Lyras, A. (in review). Sport for Development and Peace (SDP) movement for refugee youth in a new society: The role of acculturation in SDP programs. Paper submitted to European Journal for Sport and Society.

\section{WORKS IN PROGRESS}

1. Ha, J. P., Choi, C. H., \& Greenwell, T. C. (manuscript in development). Online sport consumption motives: Why does an ethnic minority group consume sports in a native and host country through the Internet? To be submitted International Journal of Sport Marketing and Sponsorship.

2. Ha, J. P. (manuscript in development). The relationship between organizational justice and organizational commitment: The moderating effect of group cohesion. To be submitted International Journal of Sport Management.

3. Ha, J. P., \& Hums, M. A. (data should be collected). Resource allocation between Olympic and Paralympic athletes by the United States Olympic Committee.

4. Kang, S. J., Ha, J. P., \& Hambrick, M., \& (data collection in completion). Smartphone in sport: What motivate people to use sport-related mobile applications?

5. Ha, J., \& Ha, J. P. (First draft was finished). The mediating role of spectator satisfaction in the relationship between affective reaction and intention to attend a game. To be submitted to Journal of Contemporary Athletic.

\section{REFERRED CONFERENCE PAPERS AND PRESENTATIONS}


1. Kang, S. J., Ha, J. P., Hambrick, M., \& Greenwell, T. C. (2012, October). A conceptual model for sport-related mobile application. Submitted to the 2012 Sport Marketing Association, Orlando, FL.

2. Ha, J. P., Choi, C. H., \& Greenwell, T. C. (2012, June). Online sport consumption motivation difference between sports in homeland and those in a host country. To be presented at the 2012 North American Society for Sport Management, Seattle, WA.

3. Kang, S. J., Ha, J. P., \& Hambrick, M. (2012, June). Smartphone in sport: Examining motivations of smartphone users for sport-related mobile applications. To be presented at the 2012 North American Society for Sport Management, Seattle, WA.

4. Han, K., Ha, J. H., \& Ha, J. P. (2012, March). Satisfaction and commitment of intercollegiate student-athletes. Submitted the 2012 National Intramural Recreational Sports Association (NIRSA) Conference, Tampa, FL.

5. King, K. M., Burnett, S. W., Larimore, K., Ha, J. P. (2012, March). Multisite, physical activity, and nutrition interventions for rural, lowsocioeconomic children. To be presented the 2012 American Alliances for Health, Physical Education, Recreation and Dance (AAHPERD), Boston, MA.

6. Ha, J. P., Ha, J. H., \& Greenwell, T. C. (2011, October). Acculturation and ethnic identity in spectator sports: The linkages among acculturation, ethnic identity, points of attachment, and consumption behavior. Presented at the 2011 Sport Marketing Association (SMA) Conference, Houston, TX.

7. Ha, J. P., \& Greenwell, T. C. (2011, October). Testing American football consumption model of Asians in the United States: Using acculturation and ethnic identity. Presented at the 2011 Sport Marketing Association (SMA) Conference, Houston, TX.

8. Ha, J. P., Park, S. H., Greenwell, T. C., \& Hums, M. A. (2011, June). The role of acculturation in the American football consumption behavior of Asian population. Paper presented at the 2011 North American Society for Sport Management, London, Ontario, Canada.

9. Weinberg, W. T., Wooten Burnet, S. C., Lund, J., \& Ha, J. P. (2011, April). Teacher candidates' perceptions of their ability to meet beginning 
standards. Papers presented at the 2011 American Alliances for Health, Physical Education, Recreation and Dance (AAHPERD), San Diego, CA.

10. Benson, P. B., King, K. M., Gehrke, T. A., Ha, J. P., Dunham, K., Kommor, P. K., Kolander, C. A., \& Britt, D. (2011, April). Green Health Initiative: Implementation of a university employee wellness program. Papers presented at the 2011 American Alliance for Health, Physical Education, Recreation and Dance (AAHPERD), San Diego,CA.

11. Park, S. H., Ha, J. P., \& Kim, Y. (2010, October). A different conceptual approach to the understanding of sport fan behavior using the curiosity concept. Paper presented at the 2010 Sport Marketing Association (SMA) Conference, New Orleans, LA.

12. Ha, J. P., \& King, K. M. (2010, November). The Qualitative Comparative Analysis (QCA) of after-school physical activity participation time among middle school students. Paper presented at the 2010 Kentucky Alliance for Health, Physical Education, Recreation and Dance (KAHPERD), Lexington, KY.

13. Ha, J. P., \& Naeger, D. J. (2010, November). Dual role of Korean Physical Education Teacher. Paper presented at the 2010 Kentucky Alliance for Health, Physical Education, Recreation and Dance (KAHPERD), Lexington, KY.

14. Park, S. H., Ha, J. P., \& Kim, Y. G. (2010, June). The relationship between intellectual curiosity and intention to watch a novel sport: The moderating effect of sport media consumption. Paper presented at the 2010 North America Society for Sport Management (NASSM), Tampa, FL.

15. Hancock, M., Lyras, A., \& Ha, J. P. (2010, June). Global assessment of sport for

development program for girls and women. Paper presented at the 2010 North American Society for Sport Management (NASSM), Tampa, FL.

16. Ha, J. P. (2010, March). Is it fair to say that Paralympic athletes should receive the same amount of resources as Olympic athletes from the U.S. Olympic Committee? Paper presented at the 2010 Graduate Research Symposium, Louisville, KY. 
1. Ha, J. P. (2011). Travel Grant For the 2011 Sport Marketing Association (SMA) Conference. Funded by the University of Louisville Graduate Student Council in the amount of $\$ 350$.

2. Ha, J. P., Greenwell, T. C., Hambrick, M., \& Hums, M. A. (2011). The role of acculturation and ethnic identity in understanding Asians' Sport Consumption Behavior in the United States. Submitted to the University of Louisville's College of Education and Human Development in the amount of $\$ 4,420$ (unfunded).

3. Ha, J. P. (2011). Travel Grant for the 2011 North American Society for Sport Management (NASSM). Funded by the University of Louisville College of Education and Human Development in the amount of \$231.

4. Ha, J. P. (2010). Travel Grant for the 2010 Sport Marketing Association (SMA) Conference. Funded by the University of Louisville Graduate Student Council in the amount of $\$ 300$.

5. Ha, J. P. (2010). Travel Grant for the 2010 North American Society for Sport Management (NASSM). Funded by the University of Louisville College of Education and Human Development in the amount of $\$ 300$.

6. Lyras, A., Hancock, M., \& Ha, J. P. (2009). Olympism, peace and development: Governance, theory and practice. Grant proposal submitted to International Olympic Committee Studies Center in the amount of 15,000CHF. (unfunded)

\section{IVITED PRESENTATIONS/GUEST LECTURER}

1. Ha, J. P. (2010, March). Sports in South Korea. Presentation to SPAD 561 International Sport, University of Louisville, Louisville, KY.

2. Ha, J. P. (2010, September). Governing structure of Olympics and Paralympics. Presentation to SPAD 390 Sport Governance, University of Louisville, Louisville, KY.

3. Ha, J. P., \& Lyras, A. (2011, April). Sport for development program for refugee youth in a host country. Presented at Olympian I.D.E.A.L.S Festival in The Muhammad Ali Institute for Peace and Justice, Louisville, KY. 
4. Ha, J. P. (2011, September). Marketing Principles Applied to Sport Management. Presentation SPAD 281Principles of Sport Management. University of Louisville, Louisville, KY.

5. Ha, J. P. (2011, October). Governing structure of international sport. Presentation to SPADD 390 Sport Governance. University of Louisville, Louisville, KY.

\section{SERVICE ACTIVITIES}

Reviewer (2011). Kentucky Alliance for Health, Physical Education, Recreation and Dance (KAHPERD) Journal

Reviewer (2012). The Spring Research Conference, University of Louisville, College of Education.

Assisted with the department's Commission on Sport Management Accreditation (COSMA) Certification, University of Louisville (2011), Sport Administration program.

Student Representative for Faculty Search Committee, University of Louisville (2011), Sport Administration program

Assisted with technical and content writing for Winsch, B. J. (2010). Taking the Pulse of Student Health Needs in America: The role of school nurses in improving student health and academics: White Paper. Prepared for Superintendent Berman in Jefferson Country Public School, Louisville.

\section{PROFESSIONAL MEMBERSHIP}

North American Society for Sport Management (NASSM), Member, 2007 present

Sport Marketing Association (SMA), Member, 2010 - present

Korea Institute of Sport Science (KISS), Member, 2008 - present

Korean Society for Sport Management (KSSM), Member, 2007 - present

\section{VOLUNTEER AND COMMUNITY INVOLVEMENT}

2011 NASCAR Events at Kentucky Speedway, Sparta, KY, July , 2011 
- Assisted in improving fan communications and at-event experiences

- Helped taking surveys from spectators in the event

Volunteer for Korean Saehan Church, Louisville, KY, Aug. 2010 - Mar. 2011

- Assisted in providing foods and rides for refugees from Southeast Asia (e.g., Karen, Nepal)

Vice President of Korean Student Association, University of Louisville, Aug. 2009 - Aug. 2010

- Managed and organized annual events for Korean students and Korean American students.

Volunteer for Arcadia Community Center, Louisville, KY, Aug. 2007 - Dec. 2007

- Assisted in creating a sport and recreational manual for the refugee center

\section{HONORS AND AWARDS}

Outstanding International Student Award. International Student Center, University of Louisville, October 2008

Superintendent Award for Outstanding Service. Office of Gyeongsangnamdo Education, Korea, 2005

Scholarships for Academic Excellence. College of Education, Gyeongsang National University, Korea, 1999 - 2002. 